\title{
Synthesis and Characterization of Tin and Germanium Based Semiconductor Nanocrystals
}

\author{
by
}

$$
\text { Ying Xu }
$$

\author{
A thesis \\ submitted to the Victoria University of Wellington \\ in fulfilment of the requirements for the degree of \\ Doctor of Philosophy \\ in Chemistry
}

Victoria University of Wellington 



\section{Abstract}

Inorganic nanomaterials are being actively researched due to their unique physical and chemical properties. These materials can be used for a wide variety of applications and technologies which have stimulated research into the discovery, understanding and control of the morphology of materials at the nanoscale. Biologists have recently integrated biomaterials with semiconductor nanoparticles to expand their applications to include biosensing, bioimaging and therapeutic strategies. Since the water solubility of semiconductor nanoparticles is crucial for bioapplications, the fabrication of water-soluble semiconductor nanocrystals with tailored properties has become more significant.

This thesis is focused on the solution phase synthesis of nanoparticles and nanowires containing the element tin. This includes tin nanoparticles, tingermanium alloy nanowires, tin sulphide nanoparticles and tin telluride nanoparticles. The aim of this research was to synthesize nanocrystals with tightly controlled size and shape for various applications,in particular for bioapplications. The properties, potential applications and crystal structure of target materials have been discussed in Chapter 1.

The target materials synthesized by using chemical reaction in the presence of surfactant were characterized primarily by Transmission Electron Microscopy 
(TEM), Energy Dispersive X-ray Spectroscopy (EDX) and Selected Area Electron Diffraction (SAED). Powder X-ray Diffraction (XRD), Scanning Transmission Electron Microscopy (STEM), Scanning Electron Microscopy (SEM), UltravioletVisible Microscopy Absorption (UV-VIS), Fourier Transform Infrared (FTIR), Photoluminescence (PL) and Diffuse Reflectance were also used extensively (Chapter 2).

The third chapter of this thesis focuses on the the development of a facile and cheap route for the synthesis of tin nanoparticles by reducing a tin precursor in an organic solvent. The low-melting tin nanoparticles have been considered as a good catalyst for the growth of semiconductor nanowires.

The fourth chapter in this thesis focuses on the development of a convenient synthesis of tin germanium alloy nanowires via solution-liquid-solid growth (SLS). Tin germanium alloy nanowires were synthesized through a self-catalyzed process in which the wires were grown from in situ made Sn droplets and $\mathrm{Ge}(\mathrm{Ph})_{3} \mathrm{Cl}$. The factors affecting morphology were ascertained and the growth direction, composition, local crystal structure and possible growth mechanism have been investigated.

The fifth chapter in this thesis focuses on the development of a novel one-pot synthesis of water-soluble SnS nanoparticles. The synthesis of SnS nanoparticles involves the reaction of inorganic starting materials $\mathrm{SnBr}_{2}$ and $\mathrm{Na}_{2} \mathrm{~S}$ in the presence of various ethanolamine derivatives in ethylene glycol. Optical studies of as synthesized SnS nanoparticle show size dependent effects in both absorbance and reflectivity.

The sixth chapter in this thesis focuses on the development of a facile direct 
synthesis of water dispersible SnTe nanoparticles. The optical properties of prepared SnTe nanoparticles were determined.

The final chapter in this thesis summarizes the main findings of this study and draws out recommendations for future work. In this study, some novel contributions have been made to produce facile one-pot synthesis of tin germanium nanowires and water soluble, size controlled tin chalcogenides nanoparticles. The main future work for tin germanium alloy naowires is to develop the method to produce nanowires without seed nanoparticles for optoelectronics applications. Further work is also needed to optimize the water synthesis of SnTe nanoparticles. 



\section{Acknowledgments}

I would firstly like to thank my supervisors Richard Tilley and Najeh Al-Salim for all their continual support and advice throughout this work. Without them this project could not have been completed. My sincere thanks also go to my colleagues in the Tilley's research group, especially Khadijah Kumaradin, Peter Ferguson, Conrad Lendrum and John Beal.

I would also like to thank all the general staff and students of the School of Chemical and Physical Science, especially David Flynn, without his invaluable support on the Electron Microscopes this project could not have been as productive.

I am extremely grateful to Industrial Research Limited (IRL) for use of their research facility for research and also to all the staff members in the Energy and Materials Group and Nano and Micro Fluidics Group in IRL, for their encouragement and support during my study. Furthermore, I would like to thank my officemate Kathryn Graham for the duration of our studies at IRL.

For other scientific discussions and advice I would like to thank Neil Curtis, David Weatherburn, Gerald Smith, John Hamlin and Nick Strickland. My thanks also go to my supervisors Tilley and Al-Salim and to Herbert Wong, Peter Ferguson, Conrad Lendrum and John Beal for proof reading some or all 
of this thesis.

My deepest thanks goes to my family including my husband, Haizhen $\mathrm{Wu}$, my son Jack $\mathrm{Wu}$, my parents, Xianqing Jiang and Linyuan $\mathrm{Xu}$ and my parents in law Shenghe $\mathrm{Hu}$ and Chaoren $\mathrm{Wu}$, for their unreserved love and unconditional support.

I would also like to acknowledge the financial assistance of the Foundation for Research, Science and Technology through contract No IIOF VICX0601, Curtis-Gordon Research Scholarships, Victoria University of Wellington and the Industrial Research Limited.

And finally I extend my appreciation to Bruce McLennan and Pamela and Ross Fletcher for their kind offer of transportation. 


\section{Abbreviations}

CVD

DMEA

DMF

DTT

EDX

EG

FTIR

IR

MBE

MDEA

MPA

NIR

$\mathrm{OA}$

OLA

PL

PVD

SAED

SEM

SLS

STEM

TEA chemical vapour deposition.

$\mathrm{N}, \mathrm{N}$-dimethylethanolamine.

dimethyl formamide.

dithiothreitol.

Energy Dispersive X-ray Spectroscopy.

ethylene glycol.

Fourier Transform Infrared.

infrared reion.

molecular beam epitaxy.

$\mathrm{N}$-methyldiethanolamine.

3-mercaptopropionic acid.

near infrared reion.

oleic acid.

oleylamine.

Photoluminescence.

physical vapor deposition.

Selected Area Electron Diffraction.

Scanning Electron Microscopy.

Solution-liquid-solid growth.

Scanning Transmission Electron Microscopy. triethanolamine. 
TEM

TGA

THF

TOA

TOP

UV-VIS

VLS

XRD
Transmission Electron Microscopy.

thioglycolic acid.

tetrahydrofura.

Trioctylamine.

Trioctylphosphine.

Ultraviolet-Visible Microscopy Absorption. vapour-liquid-solid growth.

Powder X-ray Diffraction. 


\section{Contents}

1 Introduction 1

1.1 General Overview . . . . . . . . . . . . . . . . . . . . 1

1.2 Solution Phase Synthesis of Nanocrystals . . . . . . . . . . . . 4

1.2.1 Formation mechanism and size and shape determination $\quad 4$

1.2.2 Solution-liquid-solid growth of nanowires . . . . . . 8

1.3 Quantum Confinement in Semiconductors . . . . . . . . . . 9

1.3.1 Band structure of semiconductors . . . . . . . . . 10

1.3.2 Quantum confinement ............... 11

1.4 Tin nanoparticles . . . . . . . . . . . . . . . . . 12

1.5 Tin-Germanium alloy nanowires . . . . . . . . . . . . 15

1.6 Tin monosulphide . . . . . . . . . . . . . . . . . . . . 19

1.7 Tin telluride ........................ 22

$\begin{array}{lr}\text { REFERENCES } & 24\end{array}$

2 Characterization Techniques $\quad 31$

2.1 Transmission Electron Microscopy . . . . . . . . . . . . . . 32

2.1.1 Basic Principle of TEM . . . . . . . . . . . . . . 32

2.1.2 Limitations of TEM . . . . . . . . . . . . 35

2.1 .3 Image Interpretation $\ldots \ldots . \ldots . \ldots$

2.1.4 Characterization using TEM Images . . . . . . . . . . 37

2.1.5 Characterization Using Electron Diffraction Pattern . . . 43 
2.2 Scanning Electron Microscopy . . . . . . . . . . . . . . . . . . 45

2.3 Energy Dispersive X-ray Spectrometry . . . . . . . . . . . . . 49

2.3.1 Qualitative Analysis . . . . . . . . . . . . . . . . 50

2.3.2 Quantitative Analysis . . . . . . . . . . . . . . 53

2.4 Powder X-ray Diffraction $\ldots \ldots \ldots \ldots \ldots$

2.4 .1 Phase Identification . . . . . . . . . . . . . . . . . 54

2.4.2 Determination of Alloy Composition . . . . . . . . 55

2.4.3 Determination of Crystallite Size . . . . . . . . . . . 56

2.5 Optical Properties Measurement _ . . . . . . . . . . . . . . 58

2.5.1 Ultraviolet-visible (UV-vis) spectrophotometer . . . . . 58

2.5.2 Fluorescence spectroscopy . . . . . . . . . . . . . . 60

2.5.3 Diffuse reflectance spectrometer . . . . . . . . . . 61

2.5.4 Fourier Transform Infrared (FTIR) spectrometer . . . . . 61

REFERENCES

3 Synthesis of Tin nanoparticles $\quad 65$

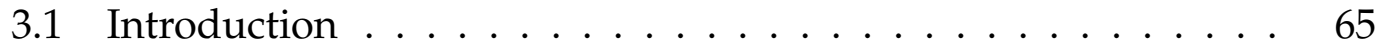

3.2 Tin nanoparticles Synthesis and Characterization . . . . . . . . 68

3.2.1 Experiment A: Reaction at $150{ }^{\circ} \mathrm{C} \ldots \ldots \ldots \ldots \ldots$

3.2.2 Experiment B-C: Effect of reaction temperature . . . . . 75

3.2.3 Experiment D: Effect of high concentration . . . . . . . 79

3.2.4 Experiment E: Effect of surfactant _ . . . . . . . . 81

3.2.5 Experiment F-G: Effect of Tin source . . . . . . . . . . . 82

3.3 Summary ...................... 86

$\begin{array}{lc}\text { REFERENCES } & 87\end{array}$

4 Solution Synthesis of Tin Germanium Alloy Nanowires 89

4.1 Introduction . . . . . . . . . . . . . . . . . . . . . 89 
4.2 Synthesis of tin germanium nanowires . . . . . . . . . . . . . 92

4.2.1 Experiment A: Reaction at $200{ }^{\circ} \mathrm{C} \ldots \ldots . \ldots 93$

4.2.2 Experiment B: Reaction at $300{ }^{\circ} \mathrm{C} \ldots \ldots . \ldots 96$

4.2.3 Experiment C: Effect of injection rate . . . . . . . . . 99

4.2.4 Experiment D: Effect of Sn/Ge precursors ratio _. . . . 100

4.2.5 Experiment E: Effect of the size of tin nanoparticle . . . . 102

4.2.6 Experiment F: Effect of Sn nanoparticles made in situ or in advance . . . . . . . . . . . . . . . 105

4.2.7 Experiment G: Effect of volume of solvent . . . . . . . . 108

4.3 Composition Analysis of tin germanium nanowires . . . . . . . 110

4.3 .1 XRD measurement . . . . . . . . . . . . . 110

4.3.2 EDX/SEM and EDX/STEM characterization . . . . . . 112

4.4 Local Structure Characterization of Nanowires . . . . . . . . . . 114

4.4.1 Growth direction analysis . . . . . . . . . . . . . . 114

4.4.2 Growing Zone Crystal Structure Study . . . . . . . . . 118

4.5 Growth Mechanism of Nanowires . . . . . . . . . . . . . . . . . 121

4.6 Summary . . . . . . . . . . . . . . . . . . . 122

$\begin{array}{lr}\text { REFERENCES } & 124\end{array}$

5 Synthesis of Tin Monosulfide Nanoparticles 129

5.1 Introduction . . . . . . . . . . . . . . . . . . 129

5.2 One pot synthesis of water-soluble SnS nanoparticles . . . . . 133

5.2.1 Experiment A-C : Effect of Surfactant . . . . . . . . . 135

5.2.2 Experiment D : Effect of injection speed of $\mathrm{Na}_{2} \mathrm{~S} \ldots 144$

5.2.3 Experiment E-G : Effect of the amount of TEA . . . . . 146

5.2.4 Experiment H-I : Effect of the reaction temperature . . 150

5.2.5 Experiment J-L : Effect of high temperature time series . 152

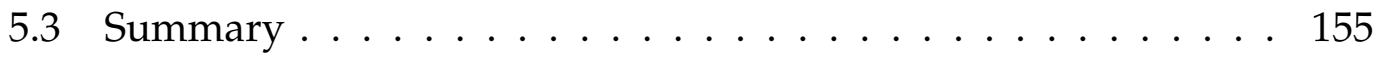


REFERENCES

6 Synthesis of Tin Telluride Nanoparticles

6.1 Introduction . . . . . . . . . . . . . . . 163

6.2 Synthesis of SnTe nanoparticles in DMF . . . . . . . . . . 168

6.2.1 Experiment A: Reaction at $50{ }^{\circ} \mathrm{C} \ldots \ldots . . . . . . .169$

6.2.2 Experiment B-C: Effect of reflux time . . . . . . . . 173

6.2.3 Experiment D-G: Effect of the amount of TEA ligand . . 178

6.3 Synthesis of SnTe nanoparticles in $\mathrm{H}_{2} \mathrm{O} \quad \ldots \ldots$

6.3.1 Experiment H-J (Effect of stabilizing ligands) . . . . . . . 188

6.3 .2 Results ...................... 189

6.3.3 Experiment K-L (Effect of $\mathrm{pH}$ condition) . . . . . . . . 192

6.4 Summary . . . . . . . . . . . . . . . . . 195

$\begin{array}{lr}\text { REFERENCES } & 197\end{array}$

7 Conclusions and Future Work $\quad 201$

7.1 Introduction . . . . . . . . . . . . . . 201

7.2 Tin Nanoparticles and Tin Germanium Nanowires . . . . . . . . 202

7.2.1 Main contributions . . . . . . . . . . . . . 202

7.2 .2 Tin nanoparticles . . . . . . . . . . . . . . . 204

7.2.3 Tin germanium nanowires . . . . . . . . . . . 205

7.2 .4 Future work . . . . . . . . . . . . . . 206

7.3 Tin chalcogenide nanoparticles . . . . . . . . . . . . 207

7.3.1 Main contributions . . . . . . . . . . . . . 207

7.3.2 Tin monosulphide . . . . . . . . . . . . 208

7.3.3 Tin telluride . . . . . . . . . . . . . . . 209

7.3 .4 Future work . . . . . . . . . . . . . . . 210 


\section{Chapter 1}

\section{Introduction}

\section{Contents}

1.1 General Overview $\ldots \ldots \ldots \ldots \ldots \ldots \ldots$

1.2 Solution Phase Synthesis of Nanocrystals $\ldots \ldots \ldots \ldots$

1.3 Quantum Confinement in Semiconductors . . . . . . . . . 9

1.4 Tin nanoparticles $\ldots \ldots \ldots \ldots \ldots \ldots \ldots \ldots$

1.5 Tin-Germanium alloy nanowires $\ldots \ldots \ldots \ldots$

1.6 Tin monosulphide $\ldots \ldots \ldots \ldots$. . . . . . . . 19

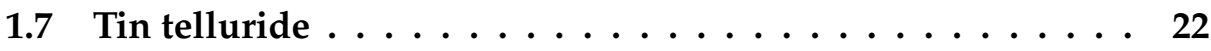

\subsection{General Overview}

Over the past few decades, nanocrystals have been the subject of enormous interest. ${ }^{1-3}$ Due to their small size in the 1-100 nm range, these nanocrystals containing a few hundred to a few thousand atoms have distinct chemical and physical properties compared to the corresponding bulk form. These properties make them ideal candidates for widespread applications in opticalelectronic devices, ${ }^{4}$ hydrogen storage devices, ${ }^{5}$ solid-state gas sensors, ${ }^{6}$ solar 
energy conversion, ${ }^{7}$ biotechnology and biomedicine. ${ }^{2,8}$ With the growing interest in creating advanced materials using these nano-building blocks, ${ }^{9}$ the development of nanocrystals has been intensively pursued. Developing new synthetic methods to provide better control over particle size, distribution, morphology, quantity and quality has been an important milestone in getting well defined and useful products. The use of economical and non toxic reagents is an increasingly important consideration in chemical synthesis.

Two different approaches have been developed to prepare nanostructures with characteristic nanoscale dimensions: (1) the traditional "top-down" approach which starts with a large block of material and by utilizing physical methods (e.g. selective etching and photolithography ) carves out the desired nanostructure; and (2) the "bottom-up" approach which starts at the atomic level and by employing chemical methods (e.g.chemical vapour deposition (CVD) and solution-liquid-solid growth (SLS)) builds nanocrystals with precisely controlled sizes and shapes. In principal, the "top-down" approach addresses crystal dimensions of larger than $50 \mathrm{~nm} .{ }^{10}$ Smaller dimensions down to $10 \mathrm{~nm}$ can be provided, but this is currently economically unviable for commercial production. ${ }^{10}$ The "bottom-up" approach provides an important complement to the "top-down" approach and readily forms nanocrystals of sizes less than $10 \mathrm{~nm}$. "Bottom-up" approaches can be performed in either vapor or solution phase. ${ }^{11}$ Vapour phase synthesis such as chemical vapor deposition (CVD), ${ }^{12}$ physical vapor deposition (PVD) ${ }^{13}$ and molecular beam epitaxy (MBE) ${ }^{14}$ have been extensively developed for high quality products, and are commonly used for metal and metal oxide nanocrystals. Compared to vapor phase synthesis, solution phase techniques offer many advantages: (1) low cost and ease of handling; (2) it involves relatively low reaction temperatures $\left(<350^{\circ} \mathrm{C}\right)$ compared to those in the vapor phase $\left(>450{ }^{\circ} \mathrm{C}\right) ;{ }^{15}$ (3) can be designed for the synthesis of 
various materials such as binary nanocrystals (SnS, SnTe). ${ }^{16,17}$ Owing to these advantages, solution phase syntheses were employed in the present work. The relevant aspects of theory concerning the growth and stabilization of colloidal nanocrystals will be explained in more detail below (see section 1.2).

In the past two decades, cadmium-based materials have been the subject of intensive research owing to their wide spread utility in variety of electronic and optoelectronic devices such as solar cells, light emitting devices and infrared detectors. ${ }^{18}$ However, these materials have potential risks to human health and the environment. ${ }^{19}$ In recent years, increasing legislation has steadily been introduced throughout the world to restrict the use of cadmium in optoelectronic applications, particularly in solar cell industry. ${ }^{18}$ Removal of cadmium-based material from optoelectronic devices has been hampered by the absence of viable alternatives that possesses similar properties. These drove the current research in the area of synthesis and characterization of new, low toxicity and low cost narrow band gap semiconductors.

In contrast, tin or germanium based materials are less toxic for humans and the environment. ${ }^{19}$ Hence, in this research, much attention has been given to the synthesis of tin or germanium-based semiconductor materials including tin germanium alloy nanowires (Chapter 4), tin monosulphide nanoparticles (Chapter 5) and tin telluride nanoparticles (Chapter 6). Moreover, the synthesis of metal tin nanoparticles (Chapter 3) has also been developed since tin nanoparticle is a good catalyst for the fabrication of tin germanium nanowire.

The general physical properties, crystal structure and potential applications of the target materials are reviewed in section 1.4-1.7 and are namely: the tin, tin germanium alloy, tin monosulfide and tin telluride. A review of synthetic 
approaches is contained in each of the introduction sections of the relevant results Chapters.

\subsection{Solution Phase Synthesis of Nanocrystals}

Solution phase synthesis has become a promising approach to prepare nanocrystals, in particular for crystal sizes of less than $50 \mathrm{~nm} \cdot{ }^{20}$ The size and shape of the colloidal nanocrystals can be controlled by varying the surfactant. Surfactants play multiple roles in nanocrystal synthesis: ${ }^{21}(1)$ they can affect the rate of nucleation and growth and consequently control the nanocrystal size; (2) they can stabilize the nanocrystals and prevent inter-particle agglomeration by increasing the steric barrier; (3) they can improve the nanocrystal solubility in conventional solvents.

Over the past decade, the formation mechanism and the size and shape determination of colloid nanocrystals have been intensively studied, ${ }^{22,23}$ and it will be discussed in the following section 1.2.1. In addition, the synthesis of nanowires developed through a solution-liquid-solid (SLS) growth mechanism will be illustrated in section 1.2.2.

\subsubsection{Formation mechanism and size and shape determination}

The formation of colloidal nanocrystals is simply considered to occur in two key steps: (1) nucleation and (2) growth. The discussion below is based on the work of Hyeon, ${ }^{24}$ Alivisatos, ${ }^{21}$ and Cheon. ${ }^{25}$

Theoretically, there is an extremely high energy barrier for spontaneous homogeneous nucleation. Nucleation does not initiate until the nucleation threshold is reached. The LaMer plot (Fig. 1.1) reveals how the energy barrier works to 
induce a "burst of nucleation". ${ }^{26}$ In stage I, some nuclei may form under supersaturated conditions of active atomic or molecular species (monomer) $(S=1$, Fig. 1.1). But these nuclei are thermodynamically unstable and are readily redissolved. In stage II, a burst nucleation is initiated by abruptly increasing the concentration of the monomer over the threshold ( $\mathrm{S}=\mathrm{Sc}$, Fig. 1.1). This may be achieved by swiftly injecting the precursor into the solvent at a relatively high temperature, such as in the hot injection method. When the monomer concentration is depleted owing to the nucleation process, the system moves into the growth stage (nucleation rate $=0$, stage III, Fig. 1.1)

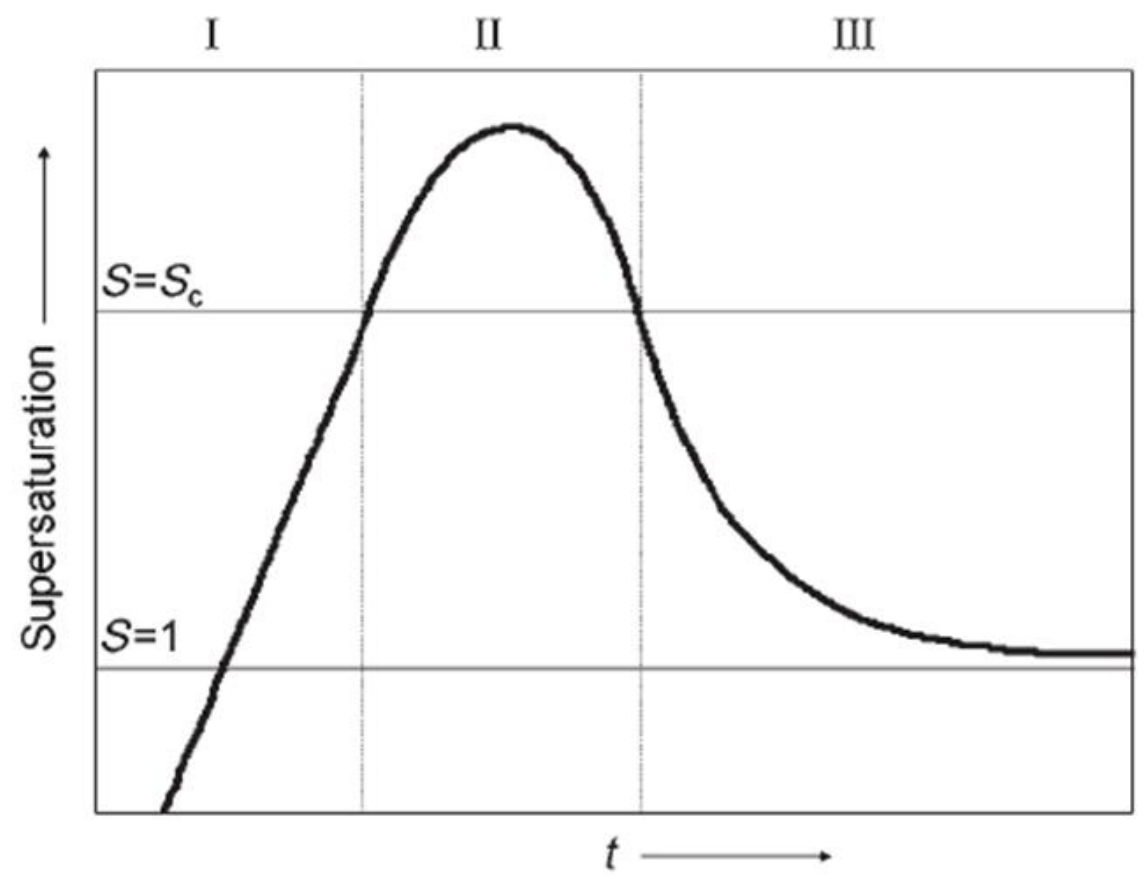

Fig. 1.1 LaMer Plot: change of degree of supersaturation as a function time $\left(\mathrm{S}=\right.$ Actual concentration/equilibrium concentration). ${ }^{26}$

The growth process of nanoparticles can be either "diffusion-controlled" or "kinetically controlled" and it is highly dependent on the concentration of the monomer present. At high monomer concentrations, the critical radius (or size), which is the minimum radius of a nucleus that can grow spontaneously 
in the supersaturated solution, is small (Fig. 1.2) so that all the particles grow. According to the "growth by diffusion" model, the growth rate of a particle is inversely proportional to its radius which results in the "focusing" effect in the particles size distribution and results in a nearly monodisperse product. In contrast, large critical radius exists at low monomer concentrations(Fig. 1.2).

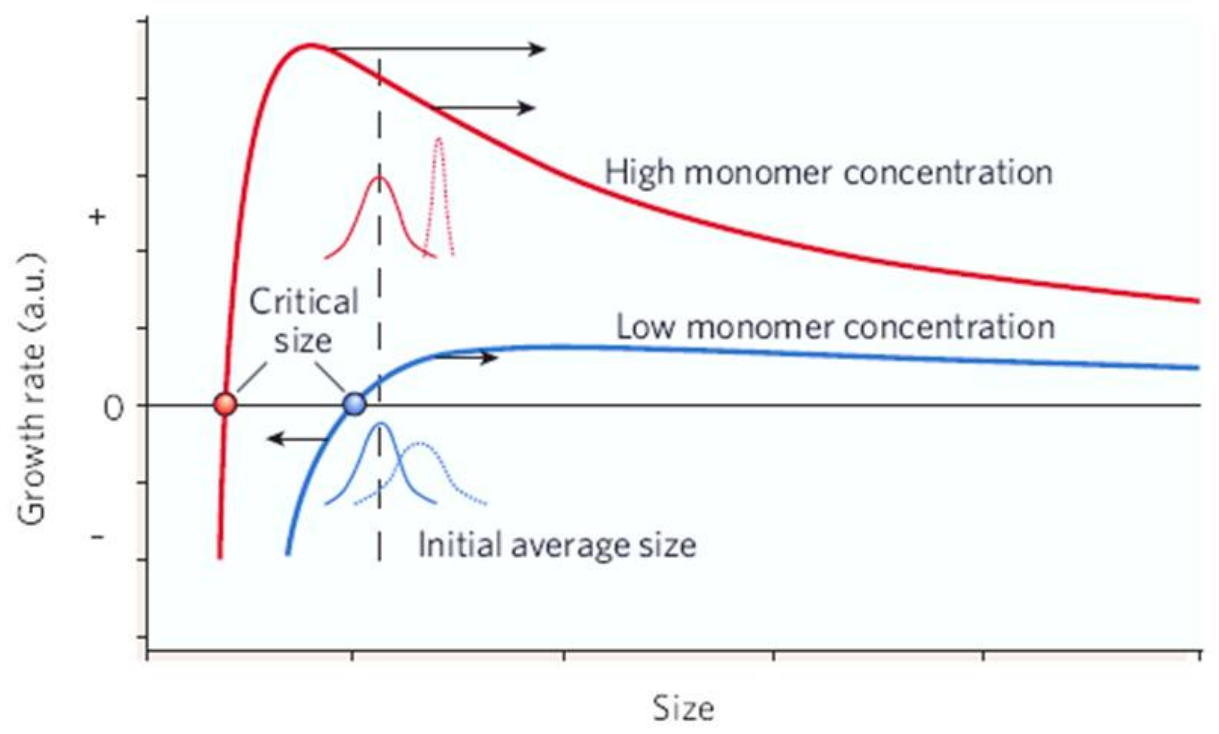

Fig. 1.2 The growth rate as a function of nanocrystal radius. ${ }^{21}$

Based on the "kinetic model (precipitation and dissolution)", small particles tend to shrink while big particles grow. This is called Ostwald ripening (Scheme 1.1), ${ }^{24}$ which leads to a "defocusing" effect in size distribution.

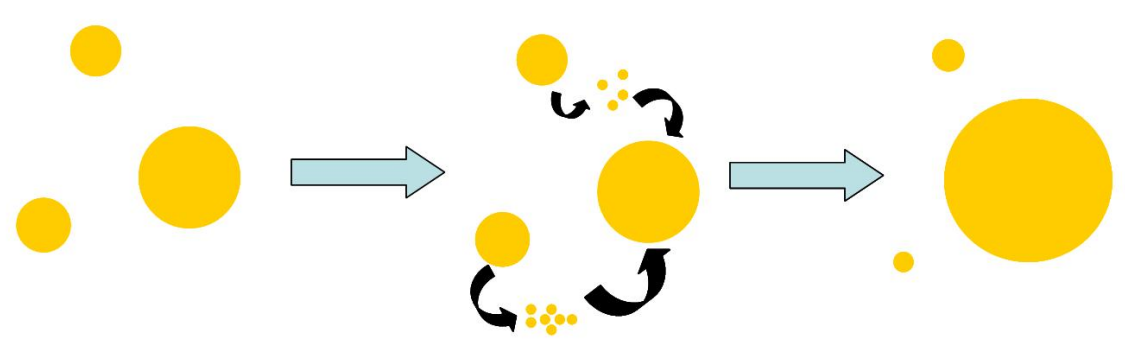

Scheme 1.1 Schematic diagrams of Ostwald ripening process. ${ }^{24}$

The geometric shape of colloidal nanocrystals is determined by three critical 
factors (Fig. 1.3): (1) the crystalline phase of the seed at nucleation stage; (2) the surface energy effect and (3) the control of the growth regime at the growth stage.

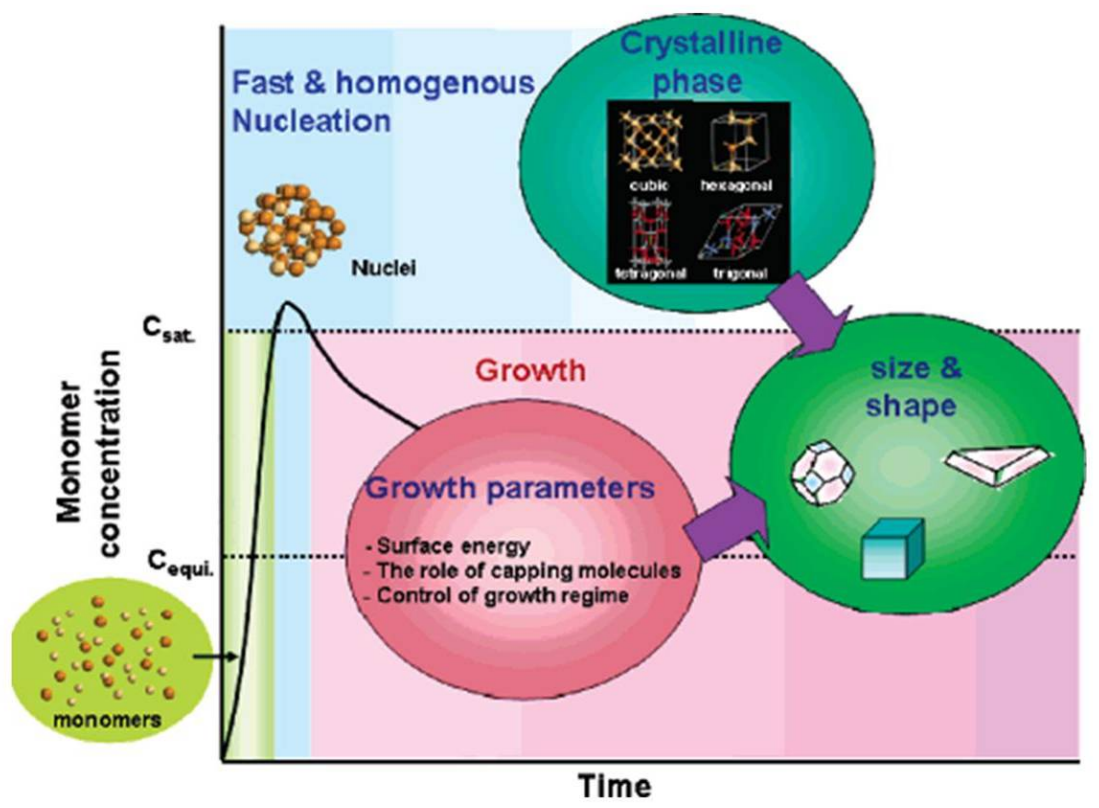

Fig. 1.3 Key factors for shape determination. ${ }^{25}$

The nucleation seed can have a variety of potential crystallographic phases from the isotropic structure of a cubic to an anisotropic structure of orthorhombic or hexagonal but the stable phase is highly dependent on its environment, such as temperature and the choice of surfactant. After the determination of a preferred crystalline phase, the subsequent growth process governs the final geometry of nanocrystals. The growth rate in different directions is strongly dependent on the surface energy of the crystallographic faces of the nucleation seed. The growth rate is exponentially proportional to the surface energy. Generally, facets with higher surface energy grow much faster than others. The surface energy of the crystal facet can be tailored by the type and the amount of adsorbed capping molecules present. For example, rock-salt 
phase PbS crystals generally nucleation as tetradecahedron seeds, exposing six $\{100\}$ faces and eight $\{111\}$ faces. The intrinsic surface energy of the $\{111\}$ faces is higher than that of the $\{100\}$ faces and hence relatively fast growth along the eight equivalent $\langle 111\rangle$ directions from cubic shaped nanocrystals. When the nanocrystals are synthesized through the thermal decomposition of organometallic precursors $\mathrm{Pb}\left(\mathrm{S}_{2} \mathrm{CNEt}_{2}\right)_{2}$ in hot organic solution in the presence of dodecylamine as nonselective capping molecules, cubic shaped PbS nanocrystals are formed. When dodecanethiol was used as capping molecules to replace dodecylamine, the surface energy of the $\{111\}$ faces can selectively be lowered relative to that of the $\{100\}$ faces. The cube shape of PbS nanocrystals are gradually changed to truncated octahedrons with the dodecanethiol concentration is increased. ${ }^{25}$ In addition, the shape of nanocrystals is also determined by the delicate balance between the kinetic and the thermodynamic growth regimes. Anisotropic growth of nanocrystals normally occurs under kinetic growth regimes while isotropic growth of nanocrystals is preferred under thermodynamic growth regimes. The control of the growth regime between kinetic and thermodynamic growth can be achieved by varying the reaction parameters such as the monomer concentration and the temperature.

\subsubsection{Solution-liquid-solid growth of nanowires}

Solution-liquid-solid (SLS) growth of nanowires was first introduced by Buhro's group in 1995 to explain the growth of InP nanowires in solution. ${ }^{27,28}$ The proposed mechanism of SLS growth shown in Fig. 1.4b is analogous to the well accepted vapour-liquid-solid growth (VLS) (Fig. 1.4a) which was first discovered by Wanger and Ellis in 1964 to explain the formation of silicon whiskers. ${ }^{29}$

In principle, the VLS growth mechanism can be considered as a three step process: (1) dissolution of the precursors by decomposition (or reduction) and 
formation of an alloy with metal nanoparticles. The alloy fully melts into a liquid droplet at the eutectic composition; (2) nucleation is initiated after the alloy reaches the supersaturated level, and then (3) followed by the axial growth of one dimensional nanowires. SLS growth is very similar to VLS growth, the difference is that vapour phase in VLS growth is substituted by a solution phase in SLS growth. In both VLS and SLS models, the melting points and reactivities of metal seeds are critically important. Generally, the seed nanoparticles must be molten under the reaction conditions and have limited solubility to dissolve target semiconductor materials to produce high supersaturation, driving one dimensional crystal growth. ${ }^{28}$

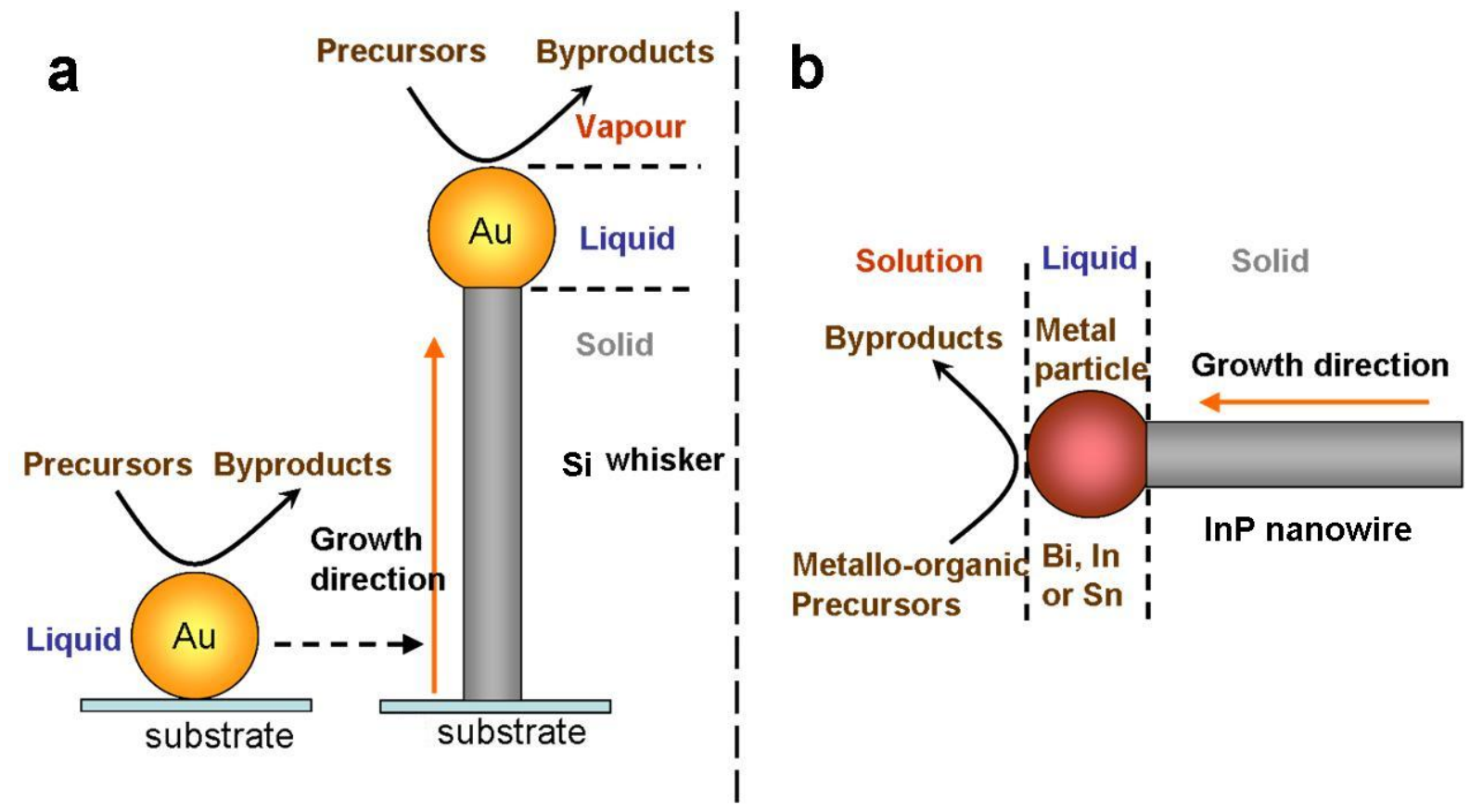

Fig. 1.4 (a) VLS growth mechanism and (b) SLS growth mechanism. ${ }^{28,29}$

\subsection{Quantum Confinement in Semiconductors}

The optical properties of nanoparticles and nanowires are affected by their particle size as a consequence of the change in band structure of nanocrystals as 
compared to its bulk properties.

\subsubsection{Band structure of semiconductors}

Semiconductors contain an "almost-empty" conduction band and an "almostfull" valence band. ${ }^{30}$ The energy difference between the bottom of the conduction band and the top of the valence bands is called the band gap energy $\left(\mathrm{E}_{g}\right)$ which can be direct or indirect band gaps, depending on the band structure. For direct band gap semiconductors, the minimum of the conduction band and the maximum of the valence band occur at momentum $\mathrm{k}=0$ ( $\mathrm{k}$ is the wave vector) as illustrated in Scheme 1.2a. ${ }^{31}$ In contrast, for indirect band gap semiconductors, the minimum of the conduction band and the maximum of the valence band are not at the same value $\mathrm{k}$ (Scheme 1.2b). ${ }^{31}$

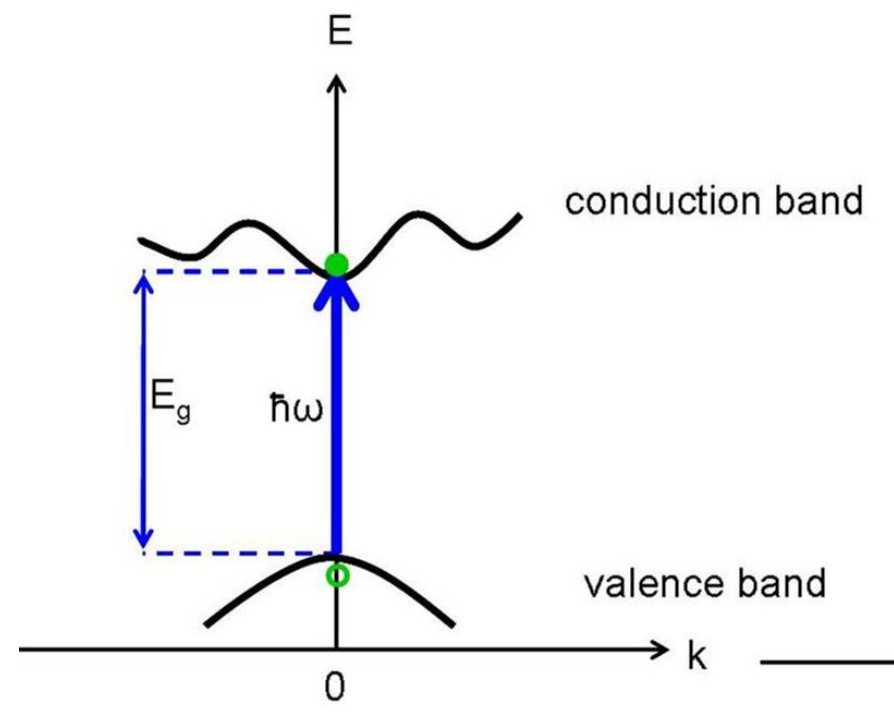

(a) Direct band gap

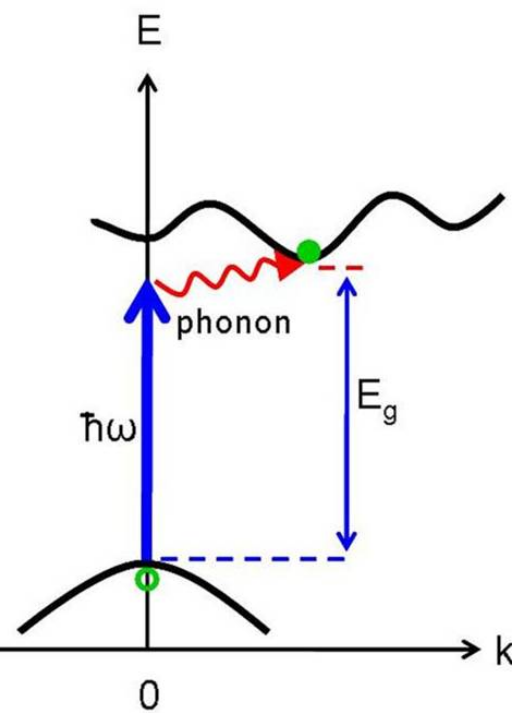

(b) Indirect band gap

Scheme 1.2 Schematic band diagrams for the absorption processes in semiconductors: (a) direct band gap, (b) indirect band gap. The vertical arrow represents the photon absorption process, while the " $\bullet$ " and "०" represent an "electron-hole" pair. The wiggly arrow in part(b) represents the absorption or emission of a phonon. ${ }^{31}$

When light with photon energy $(\hbar \omega)$ larger than the band gap energy enters 
a semiconductor, it excites an electron from the valence band into the conduction band, and generate one electron-hole pair which is called an exciton (Scheme 1.2). The distance in the electron-hole pair in bulk semiconductor, expressed as an exciton Bohr radius, provides an approximate dimension for onset of quantum confinement effects. The quantum confinement effects in semiconductor nanocrystals will be described in section 1.3.2. In fact, the absorption process in semiconductors is quite complicated. For a direct band gap process, the photon energy is sufficient to excite an electron simply straight up into a free state of almost the same $\mathrm{k}$ value (Scheme 1.2a). But, for an indirect band gap process the photons with sufficient energy to excite the electron must still fulfill the conservation of momentum which is achieved by the additional absorption or emission of a phonon as shown in Scheme 1.2b. ${ }^{31}$

\subsubsection{Quantum confinement}

Quantum confinement occurs when an electron-hole pair is squeezed into a semiconductor nanocrystal with one or more dimensions approaching the bulk exciton Bohr radius. ${ }^{32}$ The band gap of the semiconductor nanocrystals exhibits strong size dependence, which is a consequence of the quantum confinement effects. The smaller the nanocrystal size, the larger the band gap (Fig. 1.5a). macrocrystals there is conduction band and valence band and the difference in energy is called band gap. In molecules the difference in energy between HOMO and LUMO energy level is the electron transition energy which is relatively high. Nanocrystals contain limited number of molecular units and therefore the band gap energy is between that of the macrocrystals and of the molecule, i.e, larger than the crystal band gap but smaller than the molecular energy. Numerous research studies have demonstrated the blue shift in the absorption edge, ${ }^{33,34}$ reflectivity edge ${ }^{35}$ and emission ${ }^{36}$ from bulk semiconductor nanocrystal with the reduction in crystallite size. For in- 
stance, Fig. 1.5b shows emission spectra of InAs, InP and CdSe quantum dots, semiconductor nanoparticles with size less than the exciton Bohr radius. The curves demonstrate a pronounced blue shift as the quantum dot size become smaller. ${ }^{37}$

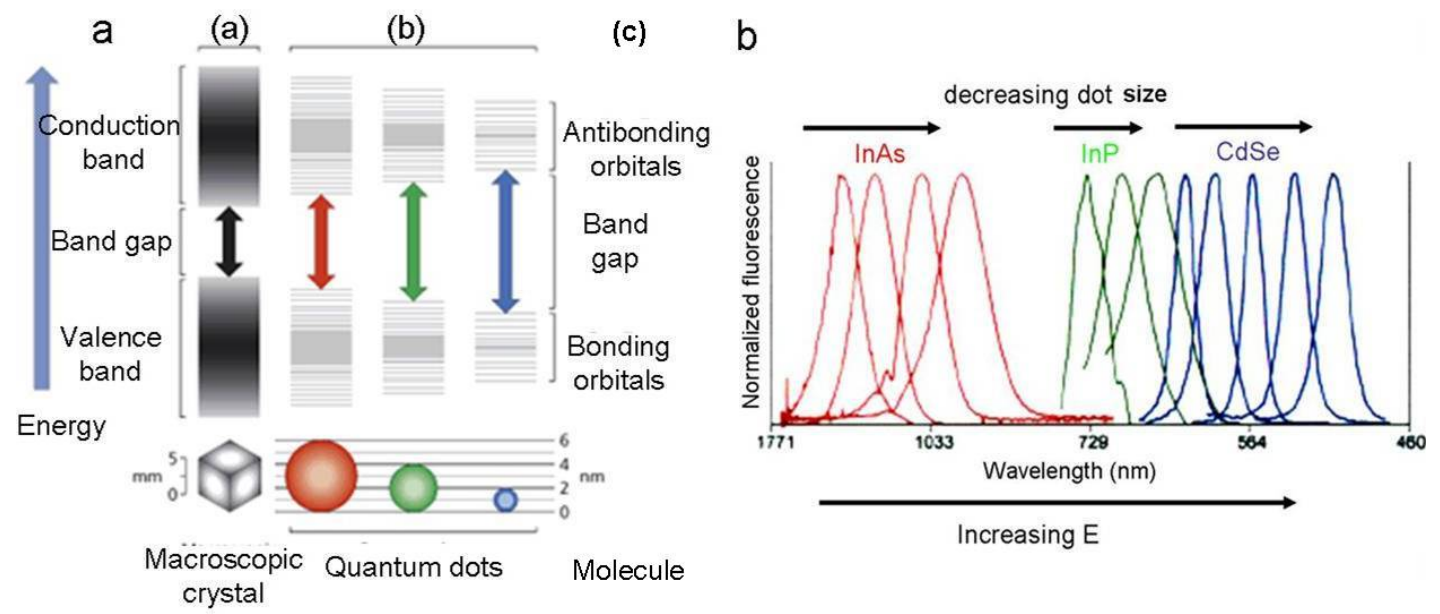

Fig. 1.5 (a) Schematic represention of the increase in the semiconductor band gap with decreasing semiconductor nanocrystal size; (b) size dependent emission spectra of InAs, InP and CdSe semiconductor quantum dots. ${ }^{37}$

\subsection{Tin nanoparticles}

\section{Physical properties and crystal structure}

Tin, a member of group IV of the periodic table, has the unusually low melting point of $232{ }^{\circ} \mathrm{C}$ and the unusually high boiling point of $2260{ }^{\circ} \mathrm{C} .{ }^{38}$ This great liquid range makes it easy to form alloys of $\mathrm{Sn}$ without loss by vaporization.

At room temperature and normal pressure, tin exists in two allotropes: One is nonmetallic $\alpha$-Sn which is stable at temperature below $13.2{ }^{\circ} \mathrm{C} .{ }^{38}$ The other is metallic $\beta$-Sn which exists between $13.2^{\circ} \mathrm{C}$ to $161^{\circ} \mathrm{C} .{ }^{38} \beta$-Sn slowly converts to $\alpha$-Sn at temperature less than $13.2^{\circ} \mathrm{C}$. This transformation was known as "Tin disease" or "Tin pest". ${ }^{39}$ When the temperature is above $13.2{ }^{\circ} \mathrm{C}, \alpha$-Sn can also 
slowly convert to $\beta$-Sn. ${ }^{39}$

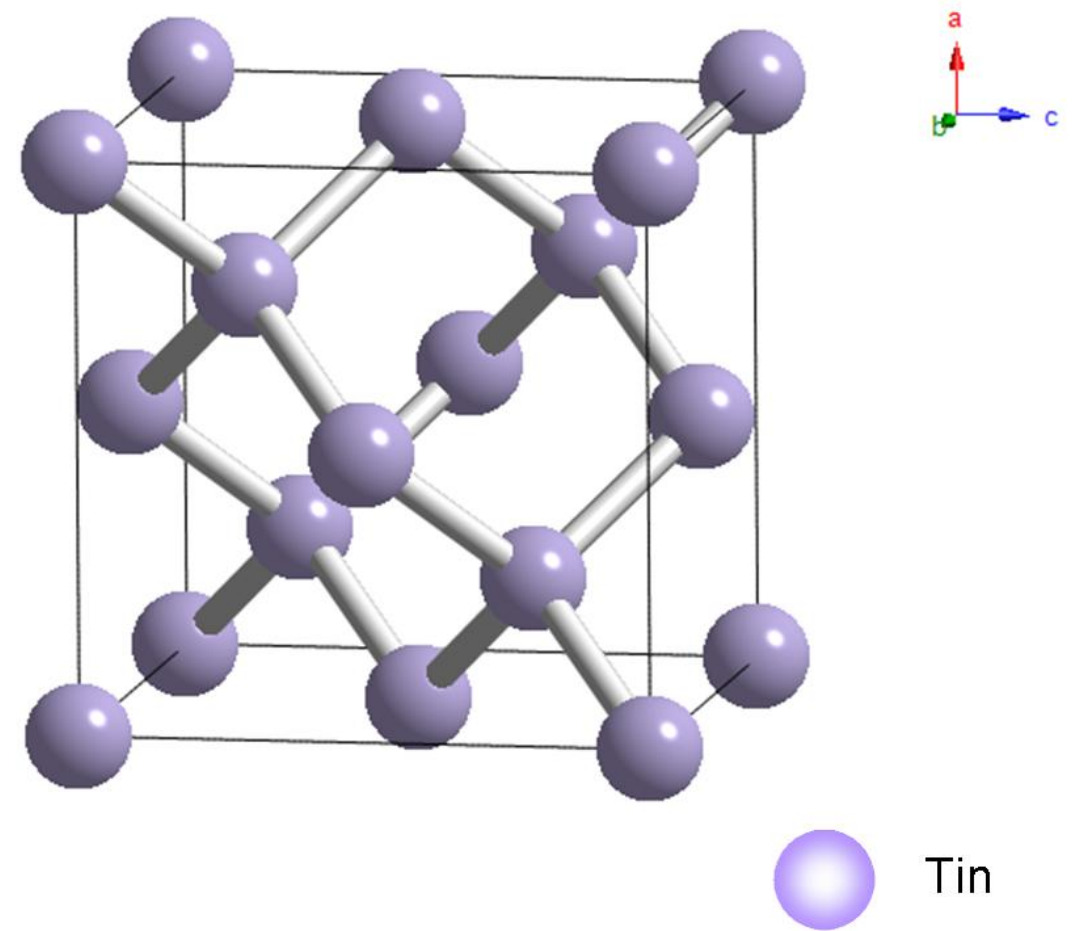

Fig. 1.6 Crystal structure of $\alpha$-Sn, diamond structure.

$\alpha$-Sn exhibits a diamond structure which consists of two interpenetrating face centered cubic Bravais lattices, displaced along the body diagonal of the cubic cell by $1 / 4$ the length of the diagonal. The lattice parameter of $\alpha-S n$ is $\mathrm{a}=6.489 \AA^{40}$ (Fig. 1.6). The main diffraction peaks of $\alpha$-Sn are shown in Table 1.1 .

Table 1.1 The main diffraction peaks of $\alpha$-Sn. ${ }^{40}$

\begin{tabular}{|c|c|c|}
\hline$(\mathrm{hkl})$ & $\mathrm{d}_{h k l}(\stackrel{\circ}{A})$ & 2theta(degree) $(\lambda=1.541 \stackrel{\circ}{A})$ \\
\hline$(111)$ & 3.751 & 27.59 \\
\hline$(220)$ & 2.294 & 45.90 \\
\hline$(311)$ & 1.956 & 54.43 \\
\hline
\end{tabular}

$\beta$-Sn has a body-centred tetragonal structure, shown in Fig. 1.7, with lattice parameters $\mathrm{a}=5.831 \stackrel{\AA}{\mathrm{c}} \mathrm{c}=3.182 \stackrel{\circ}{4}{ }^{41}$ The main diffraction peaks of $\beta$-Sn are re- 
ported in Table 1.2.

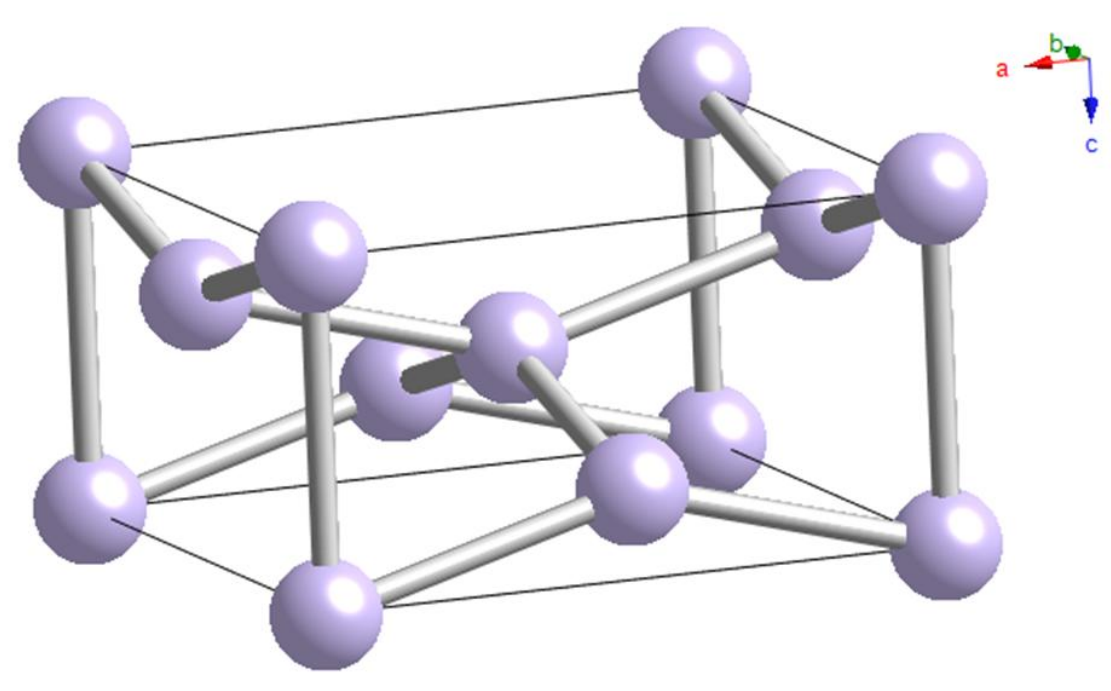

Tin

Fig. 1.7 Crystal structure of $\beta$-Sn, body-centred tetragonal structure.

Table 1.2 The main diffraction peaks of $\beta$-Sn. ${ }^{41}$

\begin{tabular}{|c|c|c|}
\hline$(\mathrm{hkl})$ & $\mathrm{d}_{h k l}(\AA)$ & 2theta(degree) $(\lambda=1.541 \AA)$ \\
\hline$(200)$ & 2.915 & 35.74 \\
\hline$(101)$ & 2.793 & 37.36 \\
\hline$(220)$ & 2.062 & 51.42 \\
\hline$(211)$ & 2.017 & 52.65 \\
\hline
\end{tabular}

\section{Potential applications}

Since $\alpha$-Sn, a gray powder, is fairly brittle, it has fewer applications. In contrast, $\beta$-Sn is a malleable, flexible and ductile metal. It resists oxygen and water and is commonly used to coat other metals to prevent corrosion. It is also considered to be non-toxic and is therefore used for food packaging, such as tin cans. Typically, tin has a low melting point $\left(232^{\circ} \mathrm{C}\right)$ and a readiness to form alloys with other metal such as lead and bismuth. ${ }^{42}$ It is an important material in solder alloys. ${ }^{43}$ 
Nanosized tin particles possess distinct properties compared with the bulk tin which have stimulated considerable interest in scientific research and technological applications. It is known that lithium ion battery performance can be enhanced by incorporating tin nanoparticles in the anode electrode, owing to the increase in interfacial area and decrease in lithium ion transport path length. ${ }^{44,45}$ The melting point of tin can decrease dramatically with particle size reduction to several nanometers owing to its enhanced surface area to volume ratio. ${ }^{46,47}$ The decreased melting point of tin nanosolder is highly desirable for preventing damage to electronic devices, caused by high reflow temperatures. ${ }^{48}$ Moreover, the low melting point metal nanoparticles have also been considered as a catalyst for the growth of semiconductor nanowires via solution liquid solid (SLS) mechanism, ${ }^{28}$ which is where the interest of this research lies.

\subsection{Tin-Germanium alloy nanowires}

\section{Physical properties and crystal structure}

Tin germanium alloy $\left(\mathrm{Sn}_{x} \mathrm{Ge}_{1-x}\right)$ is a potentially interesting group IV semiconductor material. ${ }^{49}$ It is predicted to possess a direct and tunable band gap for certain stoichiometries. ${ }^{50}$

A number of theoretical investigations of the electronic structure of $\mathrm{Sn}_{x} \mathrm{Ge}_{1-x}$ alloys have been done in the past few decades. ${ }^{49}$ For instance, using the Tightbinding model within the virtual crystal approximation (VCA), $\mathrm{Sn}_{x} \mathrm{Ge}_{1-x}$ is predicted to become a direct band gap material (Fig. 1.8), with $0.55>\mathrm{E}_{g}>0 \mathrm{eV}$ for $0.2<\mathrm{x}<0.6{ }^{49}$ Also, from pseudopotential electronic structure calculations in the VCA, a direct tunable band gap from $0.61>\mathrm{E}_{g}>0 \mathrm{eV}$ is predicted, for Sn concentration between $0.26<x<0.74 .^{51}$ 


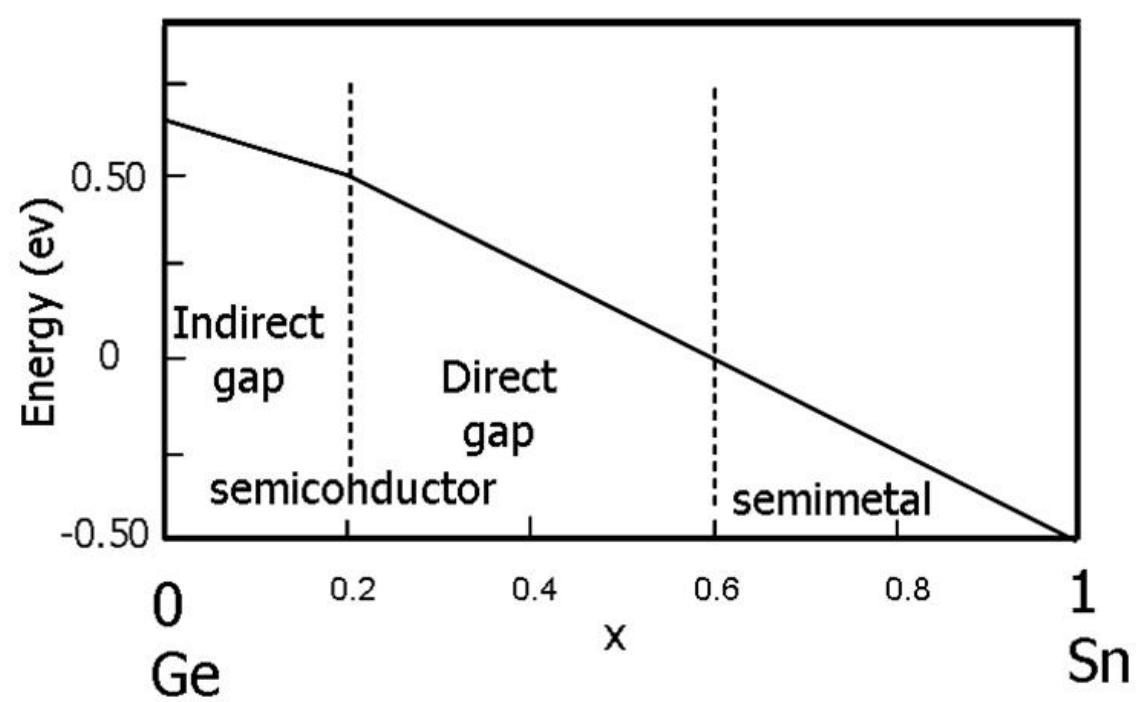

Fig. 1.8 Variation of the band gap in $\mathrm{Sn}_{x} \mathrm{Ge}_{1-x}$ alloys system. ${ }^{49}$

Recently the direct band gaps of $\mathrm{Sn}_{x} \mathrm{Ge}_{1-x}$ alloys have been experimentally measured using fast-Fourier-transform infrared interferometry (FFT-IR). ${ }^{52-54}$ $\mathrm{He}$ and Atwater reported that the direct gap varies from $0.35 \mathrm{eV}<\mathrm{E}_{g}<0.80$ $\mathrm{eV}$ for Sn concentrations range $0.15>\mathrm{x}>0 .{ }^{53}$ Vidal et al. found that the change from an indirect to a direct band gap of $\mathrm{Sn}_{x} \mathrm{Ge}_{1-x}$ alloy systems occurs at a lower critical Sn concentration $\left(\mathrm{x}_{c}: \sim 0.10\right)$ than the value predicted from theoretical calculations. ${ }^{49,51,52}$

$\mathrm{Sn}_{x} \mathrm{Ge}_{1-x}$ is a simple eutectic system (Fig. 1.9a). The eutectic temperature of $\mathrm{Sn}_{x} \mathrm{Ge}_{1-x}$ is $231^{\circ} \mathrm{C}$ which corresponds to 0.3 atomic $\%$ Ge. ${ }^{55}$ The eutectic point is $1^{\circ} \mathrm{C}$ below the melting temperature of pure bulk $\mathrm{Sn} .{ }^{55}$

The solubility of Sn in Ge and Ge in Sn in the solid state are both low (about 1 atomic \% or so). ${ }^{56}$ Previous researchers have found that Ge exhibits a relatively high solubility in liquid Sn which ranges from 1.07 atomic\% at $300{ }^{\circ} \mathrm{C}$ to 53 atomic $\%$ at $750{ }^{\circ} \mathrm{C}$ (Fig. 1.9b) However, as stated above, Ge and Sn are only miscible to about $1 \%$ in the solid state, when the alloy cools down below 
the melting point, phase separation is possible. Hence, based on the phase diagram of $\mathrm{Sn}-\mathrm{Ge}$ system in the bulk phase, the synthesis of $\mathrm{Sn}_{x} \mathrm{Ge}_{1-x}$ alloy is a significant challenge.

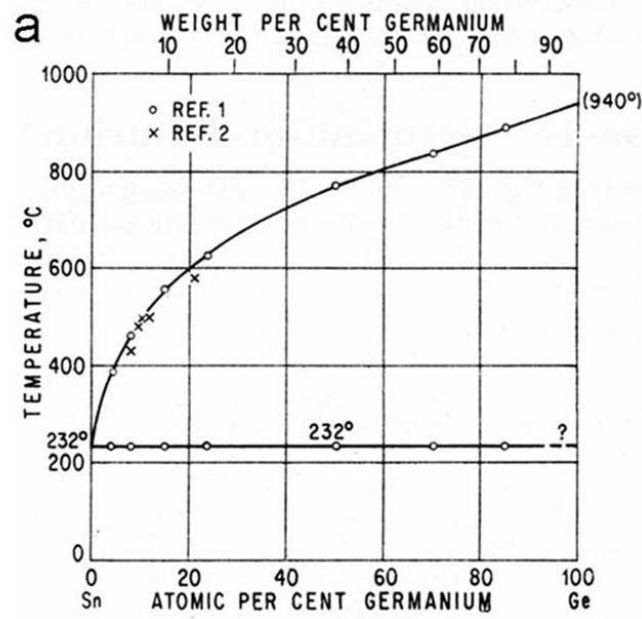

\begin{tabular}{c|c|c} 
b & \multicolumn{2}{|c}{ Solubilities in the Ge-Sn system } \\
\hline $\begin{array}{c}\text { Temperature, } \\
{ }^{\circ} \mathrm{C}\end{array}$ & $\begin{array}{c}\text { Sn in Ge } \\
\text { a/o }\end{array}$ & $\begin{array}{c}\text { Ge in liquid Sn } \\
\text { a/o }\end{array}$ \\
\hline 900 & 0.17 & \\
800 & 0.49 & \\
750 & $\ldots$. & 53 \\
700 & 0.76 & 40 \\
650 & $\ldots$. & 29 \\
600 & 0.93 & 20.5 \\
550 & $\ldots \ldots$ & 13.7 \\
500 & 1.07 & 8.9 \\
450 & $\ldots \ldots$ & 5.6 \\
400 & 1.14 & 3.45 \\
350 & $\ldots$. & 1.98 \\
300 & $\ldots$ & 1.07 \\
\hline
\end{tabular}

Fig. 1.9 (a)Binary phase diagram of Ge-Sn alloy; ${ }^{55}$ (b) the table of the solubility in the Ge-Sn system. ${ }^{56}$

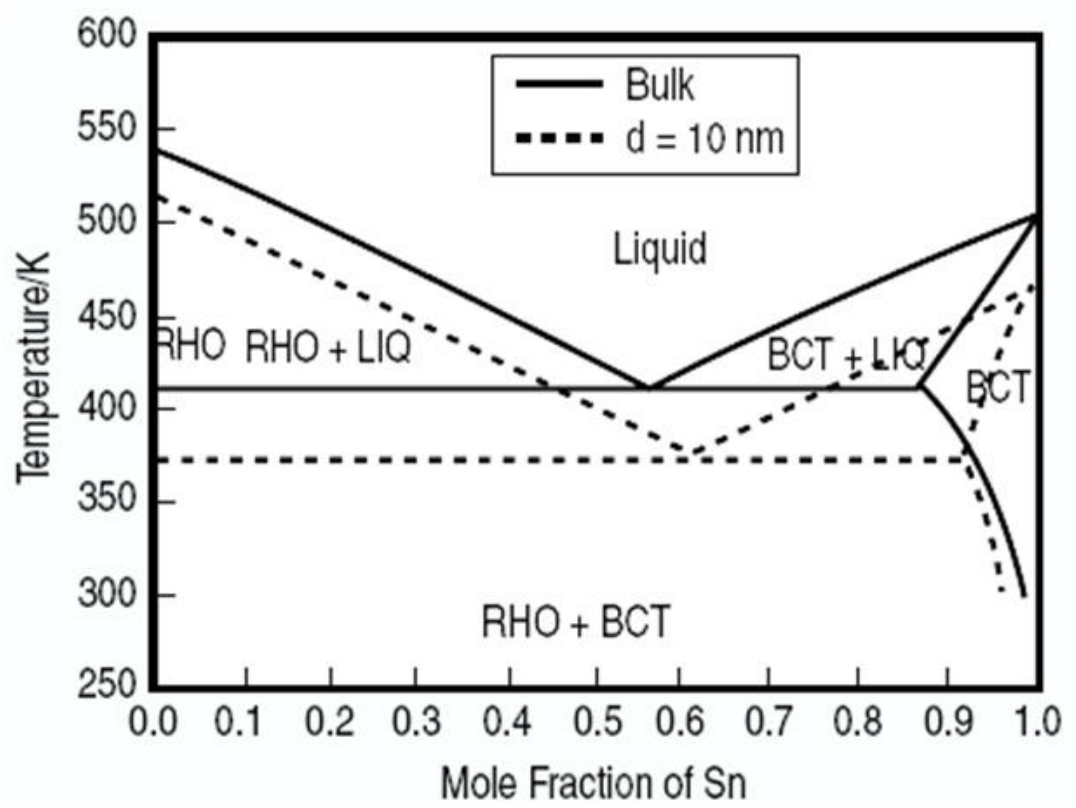

Fig. 1.10 Binary phase diagram of Bi-Sn system: the solid line shows the bulk phase; the dashed line shows the nano phase. ${ }^{57}$ (Note: RHO: Rhombohedral crystal structure for Bi; BCT: Body-centred Tetragonal crystal structure for Sn; LIQ: liquid) 
Recently, Sutter, ${ }^{58}$ Mori ${ }^{59}$ and Lee ${ }^{57}$ have demonstrated that the phase diagram of the nano-scale particle deviates significantly from that of the bulk alloy by using in situ TEM. Fig. 1.10 presents a phase diagram of the Sn-Bi system in the bulk phase (solid line) and nano phase (dashed line) which was reported by Lee and coworkers. ${ }^{57}$ It was observed that at the melting point of pure Bi and $\mathrm{Sn}$, the eutectic point of $\mathrm{Bi}_{x} \mathrm{Sn}_{1-x}$ alloy and the solid solubility of $\mathrm{Bi}$ in $\mathrm{Sn}$ in the nano phase are significantly different from the bulk phase. Mori and Lee have demonstrated that the solubility limit of $\mathrm{Sn}$ in $\mathrm{Pb}(\sim 10 \mathrm{~nm})$ is 5 times higher than the solubility limit of $\mathrm{Sn}$ in the bulk $\mathrm{Pb}$ at $110{ }^{\circ} \mathrm{C} .{ }^{59}$ Hence, it may be possible to have some difference in the solid solubility in the Sn-Ge nano phase compared with the bulk phase. Unfortunately, till now, no nano-phase diagram for Sn-Ge system has been reported.

The crystal structure of $\mathrm{Sn}_{x} \mathrm{Ge}_{1-x}$ alloy was expected to have a diamond structure. The corresponding lattice parameter $a$ of Sn-Ge alloy are assumed to obey Vegard's Law (which assumes linear interpolation between the unit cell parameters of Ge and $\alpha$-Sn). ${ }^{60}$ Previous research has demonstrated that $\mathrm{Sn}_{0.12} \mathrm{Ge} 0.88$ alloy presents a perfect diamond structure as shown in Fig. 1.11. ${ }^{61}$

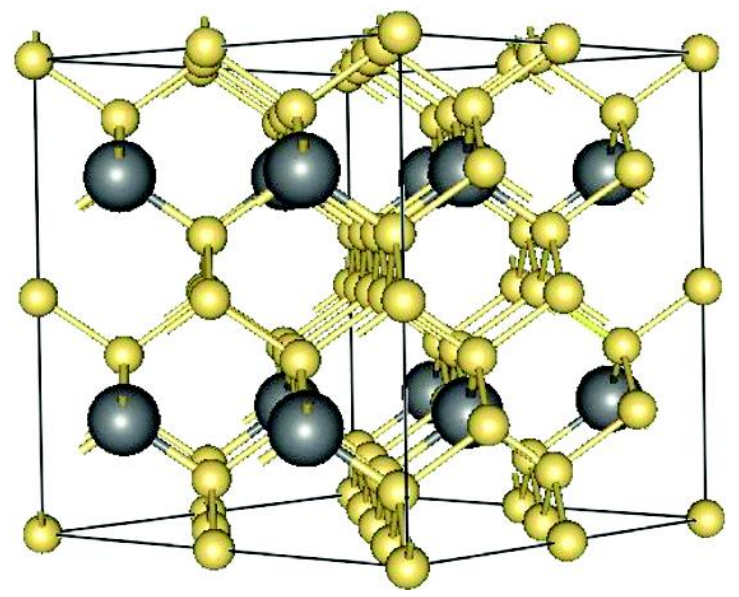

Fig. 1.11 Crystal structure of $\mathrm{Sn}_{0.12} \mathrm{Ge}_{0.88}$ alloy (diamond structure) germanium atoms: gold; tin atoms: dark gray. ${ }^{61}$ 


\section{Potential applications}

Theoretical prediction and experimental observation have suggest that $\mathrm{Sn}_{x} \mathrm{Ge}_{1-x}$ alloys may have a direct tunable band gap. ${ }^{49,53}$ These interesting optical and electrical properties make $\mathrm{Sn}_{x} \mathrm{Ge}_{1-x}$ alloys potential semiconductors for the fabrication of Si-based high performance heterojunction devices and long wavelength infrared optoelectronic devices. ${ }^{62,63}$ Furthermore, $\mathrm{Sn}_{x} \mathrm{Ge}_{1-x}$ alloys may have high carrier mobility due to small effective masses and lack of polar scattering (inherent in III-V materials). ${ }^{64}$

Recently, $\mathrm{Sn}_{x} \mathrm{Ge}_{1-x}$ alloy nanowires have attracted more and more attention for potential applications in nanoelectronic devices. ${ }^{65}$ The physical properties of $\mathrm{Sn}_{x} \mathrm{Ge}_{1-x}$ alloy nanowires may be quite different to their bulk materials due to their peculiar structure characteristics and the size effect. ${ }^{65}$

To date, some synthetic approaches have been developed for the fabrication of bulk $\mathrm{Sn}_{x} \mathrm{Ge}_{1-x}$ alloys $(\mathrm{x} \leq 0.15)$ films (see detail in section 4.1$) .{ }^{52,53}$ Investigations into synthesis of $\mathrm{Sn}_{x} \mathrm{Ge}_{1-x}$ nanowires are comparatively few. Hence, current research will focus on the synthesis of $\mathrm{Sn}_{x} \mathrm{Ge}_{1-x}$ alloys through SLS growth.

\subsection{Tin monosulphide}

\section{Physical properties and crystal structure}

Tin monosulphide (SnS), a group IV-VI semiconductor, has both an indirect band gap of $1.1 \mathrm{eV}$ and a direct band gap of $1.3 \mathrm{eV} \cdot{ }^{16,66}$ Both indirect and direct band gaps are close to the optimal band gap $(1.4 \mathrm{eV})$ for the maximum power conversion efficiency for solar cells. ${ }^{67} \mathrm{SnS}$ has shown high conductiv- 
ity (hole mobility $\left.=90 \mathrm{~cm}^{2} \mathrm{~V}^{-1} \mathrm{~s}^{-1}\right)$ and absorption coefficient $\left(\sim 10^{4} \mathrm{~cm}^{-1}\right.$, near the fundamental absorption edge) ${ }^{68}$ It possesses high conversion efficiency in photoelectric devices $(\sim 25 \%) .{ }^{69} \mathrm{SnS}$ exhibits both the p-and $\mathrm{n}$ - type conduction, depending on the concentration of tin..$^{70}$ Moreover, its constituent elements, tin and sulphur, are abundant in nature and nontoxic. ${ }^{70}$

SnS, also called Herzenbergite, was first reported by the German mineralogist R. Herzenberg in 1932. It adopts a distorted $\mathrm{NaCl}$ structure and each $\mathrm{Sn}$ atom is coordinated by six $\mathrm{S}$ atoms in a highly distorted octahedral geometry. ${ }^{71-73}$ The crystal structure (Fig. 1.12) is orthorhombic, consisting of double layers of tightly bound Sn-S atoms with Van der Waals type bonding between the layers. The lattice parameters of orthorhombic SnS are: $a=11.192 \AA, b=3.984 \AA$ and $\mathrm{c}=4.329 \AA^{\circ} .{ }^{74}$ The main diffraction peaks of $\mathrm{SnS}$ are reported in Table 1.3.

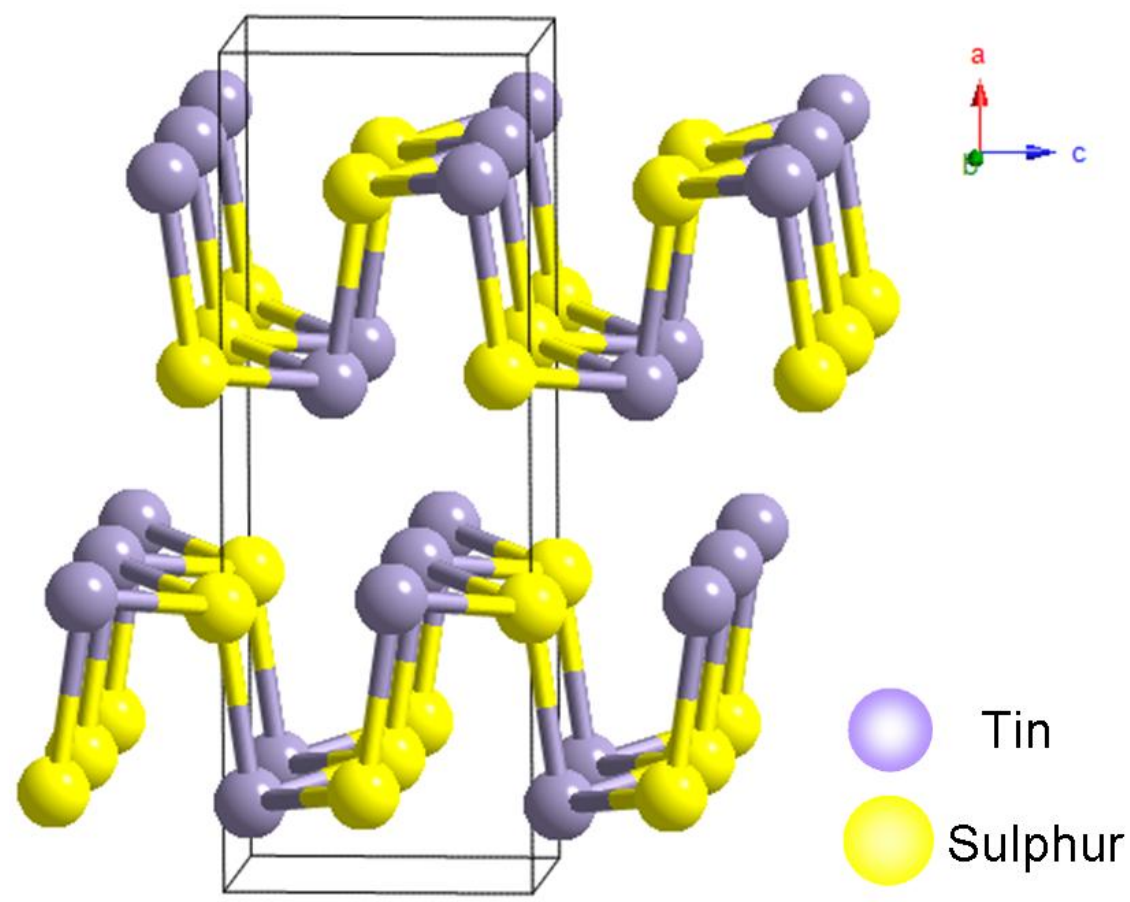

Fig. 1.12 Crystal structure of orthorhombic SnS. 
Table 1.3 The main diffraction peaks of orthorhombic SnS. ${ }^{74}$

\begin{tabular}{|c|c|c|}
\hline$(\mathrm{hkl})$ & $\mathrm{d}_{h k l}(\AA)$ & 2theta(degree) $(\lambda=1.541 \AA)$ \\
\hline$(101)$ & 4.035 & 25.62 \\
\hline$(201)$ & 3.423 & 30.30 \\
\hline$(210)$ & 3.244 & 32.01 \\
\hline$(011)$ & 2.931 & 35.54 \\
\hline$(111)$ & 2.835 & 36.78 \\
\hline$(400)$ & 2.797 & 37.30 \\
\hline
\end{tabular}

\section{Potential applications}

SnS has attracted much research interest owing to its wide spread utility in variety of electronic and optoelectronic devices, such as solar absorber in a thin film solar cell, photovoltaic materials and near-infrared detectors. ${ }^{66}$

Recently, Koktysh and coworkers reported that the indirect band gap of SnS nanoparticles with size $\sim 10 \mathrm{~nm}$ is about $1.5 \mathrm{eV}^{69}$ and Hickey et al. reported an indirect band gap of $1.6 \mathrm{eV}$ for SnS nanoparticles of size of $\sim 7 \mathrm{~nm}$. ${ }^{16}$ More recently, Liu et al. reported that SnS nanoparticles exhibit a direct band gap of 1.3 $\mathrm{eV} .{ }^{67}$ These experimental results demonstrated that SnS nanostructures possess novel optoelectronic properties compared to their bulk materials, granting them potential applications in photovoltaics, near-infrared detectors and biomedical applications. ${ }^{75,76}$

The synthesis of SnS nanoparticles has recently been developed (see detail in section 5.1) but the as-synthesized nanoparticles are mainly hydrophobic ${ }^{16}$ which to some extent limits their use in the biomedical field. ${ }^{2}$ Hence, the current research will focus on the synthesis of water soluble SnS nanoparticle with controlled size. 


\subsection{Tin telluride}

\section{Physical properties and crystal structure}

Tin telluride is a group IV-VI semiconductor. It has attracted growing attention owing to its distinctive properties and a wide range of applications. Typically, it has a narrow band gap (direct band gap of bulk SnTe of $0.18 \mathrm{eV}$ ) placing it in the infrared region, which is highly desirable for infrared optical detectors, ${ }^{77}$ thermoelectric devices, ${ }^{78}$ photo detectors, ${ }^{79}$ and radiation receivers. ${ }^{80}$ More recently, it has also been demonstrated as one of the most promising materials for thin film photovoltaics. ${ }^{17}$

SnTe adopts a cubic rock-salt (also called halite structure) crystal structure with a lattice parameter $\mathrm{a}=6.327 \AA{ }^{81}$ It can be considered as two interpenetrating face centered cubic sublattices formed respectively by Sn and Te. Each Te atom is surrounded by six Sn atoms at the corners of a regular octahedron(Fig. 1.13). The main diffraction peaks of SnTe are shown in Table 1.4.

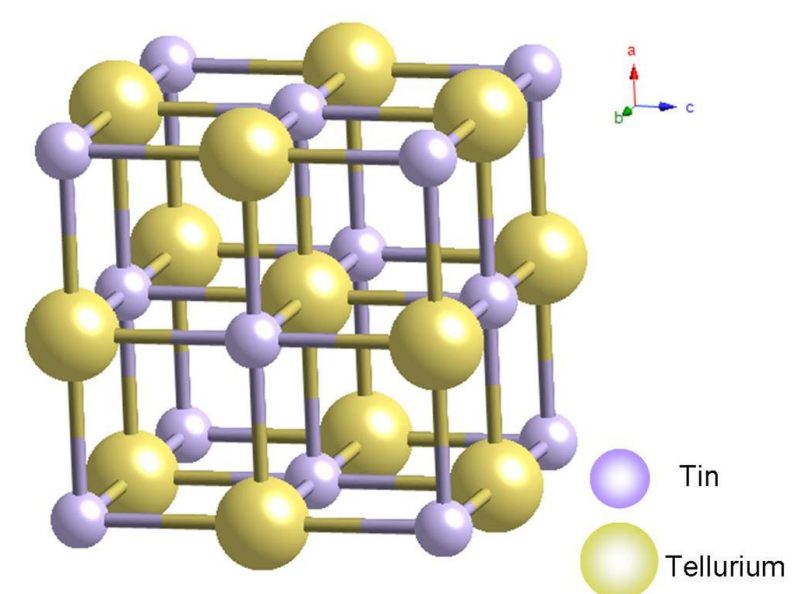

Fig. 1.13 Crystal structure of cubic rock-salt structure SnTe. 
Table 1.4 The main diffraction peaks of SnTe. ${ }^{81}$

\begin{tabular}{|c|c|c|}
\hline$(\mathrm{hkl})$ & $\mathrm{d}_{h k l}(\AA)$ & 2theta(degree) $(\lambda=1.541 \AA)$ \\
\hline$(200)$ & 3.163 & 32.85 \\
\hline$(220)$ & 2.237 & 47.139 \\
\hline$(222)$ & 1.827 & 58.642 \\
\hline
\end{tabular}

\section{Potential applications}

Experimental study has shown that the band gap of SnTe nanoparticles can be varied from $0.39 \mathrm{eV}(3185 \mathrm{~nm})$ to $0.54 \mathrm{eV}(2300 \mathrm{~nm})$ with a decrease in particle size from $14 \mathrm{~nm}$ to $7.2 \mathrm{~nm},{ }^{17}$ which could open up an even wider range of application opportunities for SnTe nanoparticles. The strong absorbance in the near-infrared region, makes SnTe nanoparticles a candidate for potential biological applications such as near-infrared thermal therapy. ${ }^{82}$

However, the research on the synthesis of SnTe nanoparticles is still relatively new (see detail in section 6.1). Current research will focus on the synthesis of water soluble SnTe nanoparticles. 


\section{REFERENCES}

[1] El-Sayed, M. A. Accounts of Chemical Research 2004, 37, 326-333.

[2] Mrinmoy, D.; Partha, S. G.; Vincent, M. R. Advanced Materials 2008, 20, $4225-4241$.

[3] Nano science and technology novel structures and phenomena; Tang, Z., Sheng, P., Eds.; London ; New York : Taylor \& Francis, 2003.

[4] Shi, L.; Xu, Y.; Hark, S.; Liu, Y.; Wang, S.; Peng, L.-m.; Wong, K.; Li, Q. Nano Letters 2007, 7, 3559-3563.

[5] Niemann, M. U.; Srinivasan, S. S.; Phani, A. R.; Kumar, A.; Goswami, D. Y.; Stefanakos, E. K. Journal of Nanomaterials 2008, 2008, 950967.

[6] Xue, X.; Xing, L.; Chen, Y.; Shi, S.; Wang, Y.; Wang, T. The Journal of Physical Chemistry C 2008, 112, 12157-12160.

[7] Yan, X.; Cui, X.; Li, B.; Li, L.-s. Nano Letters 2010, 10, 1869-1873.

[8] Wang, Y.; Tang, Z.; Kotov, N. A. Materials Today 2005, 8, 20 - 31.

[9] Carlson, J. C. T.; Jena, S. S.; Flenniken, M.; Chou, T.-f.; Siegel, R. A.; Wagner, C. R. Journal of the American Chemical Society 2006, 128, 7630-7638.

[10] Joachim, P. S. Angewandte Chemie International Edition 2002, 41, 3359-3362.

[11] Wang, Y.; Xia, Y. Nano Letters 2004, 4, 2047-2050.

[12] Lim, S. K.; Tambe, M. J.; Brewster, M. M.; Gradecak, S. Nano Letters 2008, 8, 1386-1392.

[13] Baik, J. M.; Kim, M. H.; Larson, C.; Yavuz, C. T.; Stucky, G. D.; Wodtke, A. M.; Moskovits, M. Nano Letters 2009, 9, 3980-3984. 
[14] Ramdani, M. R.; Gil, E.; Leroux, C.; Andre, Y.; Trassoudaine, A.; Castelluci, D.; Bideux, L.; Monier, G.; Robert-Goumet, C.; Kupka, R. Nano Letters 2010, 10, 1836-1841.

[15] Weintraub, B.; Zhou, Z.; bc, Y. L.; Deng, Y. Nanoscale 2010, 10, 1039.

[16] Hickey, S. G.; Waurisch, C.; Rellinghaus, B.; Eychmuller, A. Journal of the American Chemical Society 2008, 130, 14978-14980.

[17] Kovalenko, M. V.; Heiss, W.; Shevchenko, E. V.; Lee, J.-S.; Schwinghammer, H.; Alivisatos, A. P.; Talapin, D. V. Journal of the American Chemical Society 2007, 129, 11354-11355.

[18] Chandramohan, S.; Sathyamoorthy, R.; Sudhagar, P.; Kanjilal, D.; Kabiraj, D.; Asokan, K. Thin Solid Films 2008, 516, 5508-5512.

[19] Wakeham, S.; Hawkins, G.; Henderson, G.; Carthey, N. Applied optics 2008, 47, C206-C213.

[20] Zhu, Y.; Qian, Y. Science in China Series G: Physics Mechanics and Astronomy 2009, 52, 13-20.

[21] Yin, Y.; Alivisatos, A. P. Nature 2005, 437, 664-670.

[22] Wang, X.; Li, Y. Chemical Communications 2007, 2901 - 2910.

[23] Andrea, R. T.; Susan, H.; Peidong, Y. Small 2008, 4, 310-325.

[24] Park, J. P.; Joo, J.; Kwon, S. G.; Jang, Y. J.; Hyeon, T. Angewandte Chemie International Edition 2007, 46, 4630-4660.

[25] Jun, Y.-w.; Lee, J.-H.; Choi, J.-s.; Cheon, J. The Journal of Physical Chemistry B 2005, 109, 14795-14806.

[26] LaMer, V. K.; Dinegar, R. H. Journal of the American Chemical Society 1950, $72,4847-4854$. 
[27] Trentler, T. J.; Hickman, K. M.; Goel, S. C.; Viano, A. M.; Gibbons, P. C.; Buhro, W. E. Science 1995, 270, 1791-1794.

[28] Wang, F.; Dong, A.; Sun, J.; Tang, R.; Yu, H.; Buhro, W. E. Inorganic Chemistry 2006, 45, 7511-7521.

[29] Wagner, R. S.; Ellis, W. C. Applied Physics Letters 1964, 4, 89-90.

[30] Singh, B. P.; Singh, R. Electronic Devices and Integrated Circuits; Dorling Kindersley (india) Pvt Ltd, 2008.

[31] Fox, M. Optical Properties of Solids; Oxford University Press, 2001.

[32] Gaponenko, S. V. Optical Properties of Semiconductor Nanocrystals; Cambridge University Press, 1998.

[33] Anno, M., E.; Tanimoto Physical Review B 2006, 73, 155430.

[34] Pesika, N. S.; Stebe, K. J.; Searson, P. C. The Journal of Physical Chemistry B 2003, 107, 10412-10415.

[35] Berntsson, O.; Burger, T.; Folestad, S.; Danielsson, L. G.; Kuhn, J.; Fricke, J. Analytical Chemistry 1999, 71, 617-623.

[36] Xie, R.; Peng, X. Journal of the American Chemical Society 2009, 131, 1064510651.

[37] Bruchez, M. J.; Moronne, M.; Gin, P.; Weiss, S.; Alivisatos, A. P. Science 1998, 281, 2013-2016.

[38] Lide, D. R. CRC handbook of chemistry and physics, 89th ed.; CRC PR INC, 2008.

[39] Chemical principles, 6th ed.; Zumdahl, S. S., Ed.; Houghton Mifflin company, 2007. 
[40] Swanson, F. Natl. Bureau of Stand. (U.S.) Circ. 5391953.

[41] Swanson, T. Natl. Bureau of Stand. (U.S.) Circ. 539 I 1953, 24.

[42] Humpston, G.; Jacobson, D. M. Principles of soldering; ASM International, 2004.

[43] Harper, C. A. Electronic materials and processes handbook, 3rd ed.; McGrawHill, Inc., 2003.

[44] Lee, J. H.; Kong, B.-S.; Baek, Y.-K.; Yang, S. B.; Jung, H.-T. Nanotechnology $2009,20,235203$.

[45] Yu, Y.; Gu, L.; Zhu, C.; van Aken, P. A.; Maier, J. Journal of the American Chemical Society 2009, 131, 15984-15985.

[46] Buffat, P.; Borel, J.-P. Physical Review A 1976, 13, 2287-2298.

[47] Jiang, H.; Moon, K.-s.; Dong, H.; Hua, F.; Wong, C. P. Chemical Physics Letters 2006, 429, 492-496.

[48] Li, n. Y.; Moon, n. K.-s.; Wong, C. Novel Nanotechnology for Environmentally Friendly Interconnect Materials in Microelectronic Packaging Applications. Novel Nanotechnology for Environmentally Friendly Interconnect Materials in Microelectronic Packaging Applications, Los Alamitos, CA, USA, 2006; pp 32-37.

[49] Paul, W. Semiconductor Science and Technology 1990, 5, S1-S4.

[50] Bauer, M. R.; Tolle, J.; Bungay, C.; Chizmeshya, A. V. G.; Smith, D. J.; Menedez, J.; Kouvetakis, J. Solid State Communications 2003, 127, 355-359.

[51] Mader, K. A.; Baldereschi, A.; von Kanel, H. Solid State Communications $1989,69,1123-1126$. 
[52] Ladron de Guevara, H. P.; Rodriguez, A. G.; Navarro-Contreras, H.; Vidal, M. A. Applied Physics Letters 2004, 84, 4532-4534.

[53] He, G.; Atwater, H. A. Physical Review Letters 1997, 79, 1937-1940.

[54] Ragan, R.; Atwater, H. A. Applied Physics Letters 2000, 77, 3418.

[55] Hansen, M.; Anderko, K. Constitution of Binary Alloys; McGraw Hill, 1958.

[56] Elliott, R. P. Constitution of binary alloys, First supplement; McGraw Hill: New York, 1965.

[57] Lee, J.; Mori, H.; Lee, J.; Tanaka, T.; Penttil, K. JOM Journal of the Minerals, Metals and Materials Society 2005, 57, 56-59.

[58] Sutter, E.; Sutter, P. Nano Letters 2008, 8, 411-414.

[59] Lee, J. G.; Mori, H. Physical Review Letters 2004, 93, 235501.

[60] Denton, A. R.; Ashcroft, N. W. Physical Review A 1991, 43, 3161-3164.

[61] Chizmeshya, A. V. G.; Bauer, M. R.; Kouvetakis, J. Chemistry of Materials 2003, 15, 2511-2519.

[62] Pukite, P. R.; Harwit, A.; Iyer, S. S. Applied Physics Letters 1989, 54, $2142-$ 2144.

[63] Taraci, J.; Zollner, S.; McCartney, M. R.; Menendez, J.; SantanaAranda, M. A.; Smith, D. J.; Haaland, A.; Tutukin, A. V.; Gundersen, G.; Wolf, G.; Kouvetakis, J. Journal of the American Chemical Society 2001, 123, 10980-10987.

[64] Zhang, J.; Deng, X.; Swenson, D.; Hackney, S. A.; Krishnamurthy, M. Thin Solid Films 1999, 357, 85-89.

[65] Lieber, C. M.; Wang, Z. L. MRS BULLETIN 2007, 32, 99-108. 
[66] Tanusevski, A. Semiconductor Science and Technology 2003, 18, 501.

[67] Liu, H.; Liu, Y.; Wang, Z.; He, P. Nanotechnology 2010, 21, 105707.

[68] Greyson, E. C.; Barton, J. E.; Odom, T. W. Small 2006, 2, 368-371.

[69] Koktysh, D. S.; McBride, J. R.; Rosenthal, S. J. Nanoscale Research Letter $2007,2,144-148$.

[70] Zhu, H.; Yang, D.; Zhang, H. Materials Letters 2006, 60, 2686-2689.

[71] Hu, H.; Yang, B.; Zeng, J.; Qian, Y. Materials Chemistry and Physics 2004, 86, 233-237.

[72] Li, Q.; Ding, Y.; Wu, H.; Liu, X.; Qian, Y. Materials Research Bulletin 2002, $37,925-932$.

[73] Lambros, A. P.; Geraleas, D.; Economou, N. A. Journal of Physics and Chemistry of Solids 1974, 35, 537-541.

[74] Sugaki, A.; Kitakaze, A.; Kitazawa, H. author; Technical Report, 1985; p 199.

[75] Souici, A. H.; Keghouche, N.; Delaire, J. A.; Remita, H.; Etcheberry, A.; Mostafavi, M. The Journal of Physical Chemistry C 2009, 113, 8050-8057.

[76] Wang, Y.; Gong, H.; Fan, B.; Hu, G. The Journal of Physical Chemistry C 2010, $114,3256-3259$.

[77] Jeng, E. S.; Moll, A. E.; Roy, A. C.; Gastala, J. B.; Strano, M. S. Nano Letters 2006, 6, 371-375.

[78] Dresselhaus, M. S.; Chen, G.; Tang, M. Y.; Yang, R. G.; Lee, H.; Wang, D. Z.; Ren, Z. D.; Fleurial, J. P.; Gogna, P. Advanced Materials 2007, 19, 1043-1053. 
[79] Pettersson, H.; Tragardh, J.; Persson, A. I.; Landin, L.; Hessman, D.; Samuelson, L. Nano Letters 2006, 6, 229-232.

[80] Han, J.; Zhang, W.; Chen, W.; Thamizhmani, L.; Azad, A. K.; Zhu, Z. The Journal of Physical Chemistry B 2006, 110, 1989-1993.

[81] Scheer, M.; McCarthy, G.; Seidler, D.; Boudjouk, P. North Dakota State University 1994.

[82] Loo, C.; Lowery, A.; Halas, N.; West, J.; Drezek, R. Nano Letters 2005, 5, 709-711. 


\section{Chapter 2}

\section{Characterization Techniques}

\section{Contents}

2.1 Transmission Electron Microscopy $\ldots \ldots \ldots \ldots \ldots \ldots$

2.2 Scanning Electron Microscopy . . . . . . . . . 45

2.3 Energy Dispersive X-ray Spectrometry . . . . . . . . . 49

2.4 Powder X-ray Diffraction . . . . . . . . . . . 54

2.5 Optical Properties Measurement . . . . . . . . . . 58

In this thesis, solution-phase synthesis was used to prepare target nanomaterials. The prepared products were characterized using High Resolution Transmission Electron Microscopy (HRTEM), Selected Area Electron Diffraction (SAED), Scanning Electron Microscopy (SEM), Energy Dispersive X-ray Spectroscopy (EDX) and Powder X-ray Diffraction (XRD). The optical properties for nanoparticles were examined using Ultraviolet-visible (UV-VIS) spectrophotometer, Fourier Transform Infrared (FTIR) Spectrometer, Fluorescence spectroscopy and diffuse reflectance Spectrometer. 


\subsection{Transmission Electron Microscopy}

Transmission Electron Microscopy (TEM) is a microscopy technique first developed in the 1930's. It is a powerful instrument for the characterization of nanostructural materials. It affords an improved understanding of the relationship between the atomic structure of materials and their chemical and physical properties. ${ }^{1}$

\subsubsection{Basic Principle of TEM}

The basic principle of a TEM is the same as the light microscope, except that a focused beam of electrons is used instead of light to "image" the specimen. The electrons are emitted from an electron source, a V-shaped filament made of $\mathrm{LaB}_{6}$ or $\mathrm{W}$ (tungsten), accelerated by the positive potential down the column. Due to the negative potential of the filament cap, all electrons are repelled toward the optic axis. In TEM, the accelerating potential is usually several hundred thousand volts causing the electrons to travel at an appreciable fraction of the speed of light, meaning that the relativistic effects become important which are corrected by introduced an correction factor. Therefore the precise wavelength $(\lambda)$ of the electron is given by:

$$
\lambda=\frac{h}{\sqrt{2 m_{0} e U}} \times \frac{1}{\sqrt{1+\frac{e U}{2 m_{0} c^{2}}}}
$$

Where

h= Planck's constant,

$\mathrm{m}_{0}=$ mass of the electron,

$\mathrm{e}=$ elementary charge,

$\mathrm{U}=$ electric potential,

$c=$ speed of light. 
The first term in the Equation(2.1) is the non-relative expression while the last term is a relativistic correction factor. Since the wavelength of the electron is much smaller than the inter-atomic distances in crystal lattices, atomic resolution can be achieved.

Fig. 2.1 shows a ray diagram of a TEM. The electron beam is confined by condenser lens, passes the condenser aperture and strikes the specimen. Because the specimen is ultra thin, most of unscattered electrons are transmitted through the specimen with some undergoing deflection as a result of elastic scattering and inelastic scattering. Elastic scattering occurs when the incoming fast electron and an atomic nucleus interact while inelastic scattering results from the interaction between the fast electron and atomic electrons (Scheme 2.1). The unscattered and scattered electrons are then recombined by the objective lens forming either a first intermediate image or a diffraction pattern in the back focal plane. The image or the diffraction pattern will be further magnified by the intermediate lens. By altering the projector lens, the enlarged image or diffraction pattern will be focused and projected on a fluorescent screen or recorded on a digital (CCD) camera. 

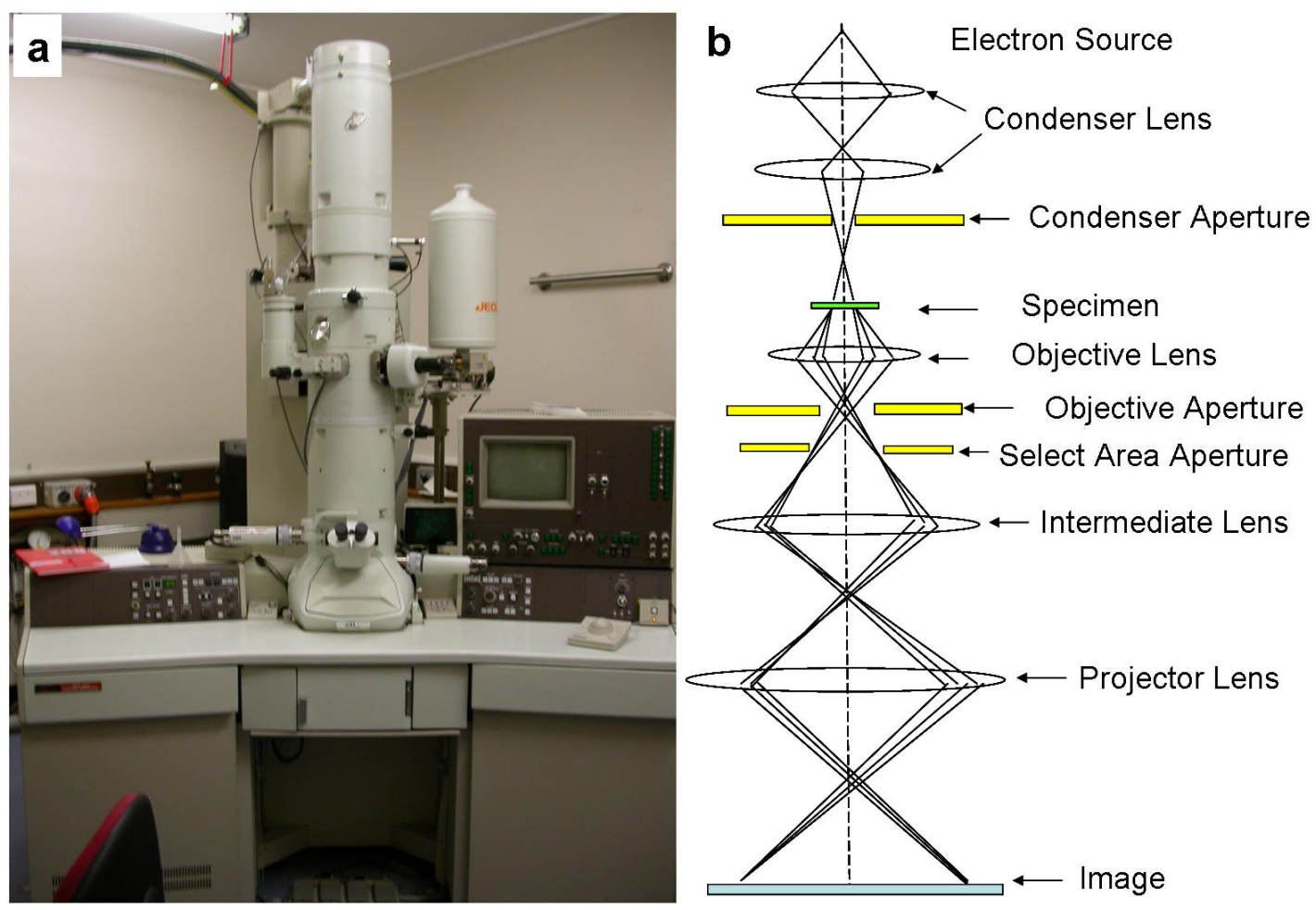

Fig. 2.1 (a) an high resolution TEM image; (b) schematic representation of the electron beam.

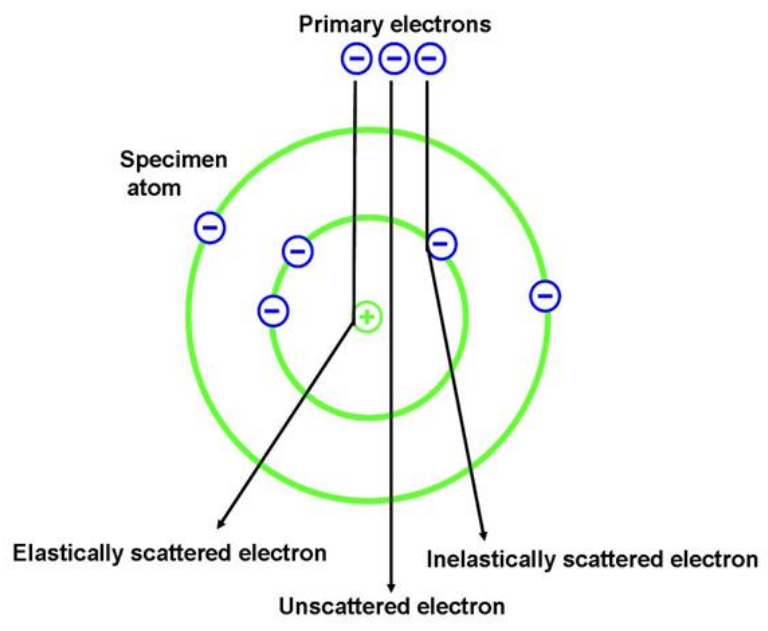

Scheme 2.1 Schematic represent the formation of elastic and inelastic scattering of electrons.

There are key advantages to the use of electrons over X-ray. The interaction of electrons with atoms is much stronger than that of X-rays with atoms. Therefore, it is possible to image individual sub-micron crystals. This could not be 
achieved by using powder X-ray diffraction. Moreover, the recombination of the diffracted beams by the objective lens during the imaging process retains the phase information, unlike the case with X-ray diffraction.

\subsubsection{Limitations of TEM}

Due to the imperfection of the microscope, there are some limits to the structural resolution that can be obtained.

\section{Objective lens aberrations}

Since the objective lens recombine the diffracted beams from the sample to form the initial image, any imperfections in the objective lens, such as two-fold astigmatism and spherical aberration, become the limiting factors. The degree and orientations of the astigmatism is caused by the departure of the lens bore from a cylindrical nature. In theory, this should be completely corrected by electromagnetic coils in the microscopes. However, the lens used in an electron microscope are the magnetic field within the vacuum between the pole-pieces of the objective lens, and the geometry of this field is hard to control. This results in a spherical aberration in which beams diffracted through different angles are brought into focus at slightly different planes. Spherical aberration causes blurring in TEM images especially at the areas near a sample edge, an interface or a defect. The blurring complicates obtaining structure information directly from the observed images. Spherical aberration can be corrected to some extent by using defocusing modulation technique.

\section{Chromatic aberrations}

The total dispersion of the energy of electrons is combined by several contributions: the initial dispersion when the electrons leave the cathode, variations of the accelerating voltage, fluctuation of the lens current and the energy loss 
resulting from the interactions of the electrons with the sample. These lead to the electron beam with a range of electron energies.

In an electron microscope, the focal length of the magnetic lens depends on the kinetic energy of electrons. That means the electrons of different energies will be focused at different focal points. And therefore, a beam of electrons with dispersed energies is not focused in a single plane by magnetic lens which results in distortion of the image formed on a single plane. This distortion is referred as chromatic aberration. Chromatic aberration affects both the contrast and resolution of fine image detail.

\section{Beam divergence}

Beam divergence occurs because the filament degrades from a point source to a larger area of electron emission and consequently the beam incident on the object is not exactly parallel. Beam divergence effects on the image resolution. Generally, the higher image resolution is obtained by the smaller divergence angle.

\subsubsection{Image Interpretation}

Structural information can be acquired both by high resolution TEM image as well as by electron diffraction. However, interpreting the structure requires sophisticated calculations which is more complex than the information obtained from X-ray diffraction. This is due to two aspects: one is the interaction between electron and specimen. The relationship between the structure and the emergent electron wavefront is more complex than the interaction between Xray and specimen, and therefore great care must be taken when interpreting the information obtained. The other aspect is the mechanics of the microscope. The ability to interpret high resolution TEM image is limited by the aberrations 
of the image forming lens.

\subsubsection{Characterization using TEM Images}

TEM images can provide morphological (size and shape), topographical (surface and interface) and crystallographic information of objects. TEM offers the unique ability to observe crystal defects at close to the atomic level as defect scatter electrons differently from their surrounding matrices. Here, the investigation of nanomaterials by TEM is briefly described. Representative examples given below are all from the present study.

In this study, JEOL 2010 TEM (Fig. 2.1a), with an accelerating voltage of 200 $\mathrm{kV}$, was employed. Samples were prepared by placing a drop of dilute, welldispersed colloidal solution onto a 200-mesh amorphous carbon-coated copper TEM grid purchased from ProSciTech. The software Gatan Digital Micrograph was used to capture and process images and performs a fast Fourier transform analysis on a high resolution TEM image.

\section{Morphological Characterization}

Fig. 2.2 shows an example of the nanoparticles formed with different shapes. Tin spheres, tin monosulphide square plates and tin monosulphide elongated hexagons were observed from the images. 

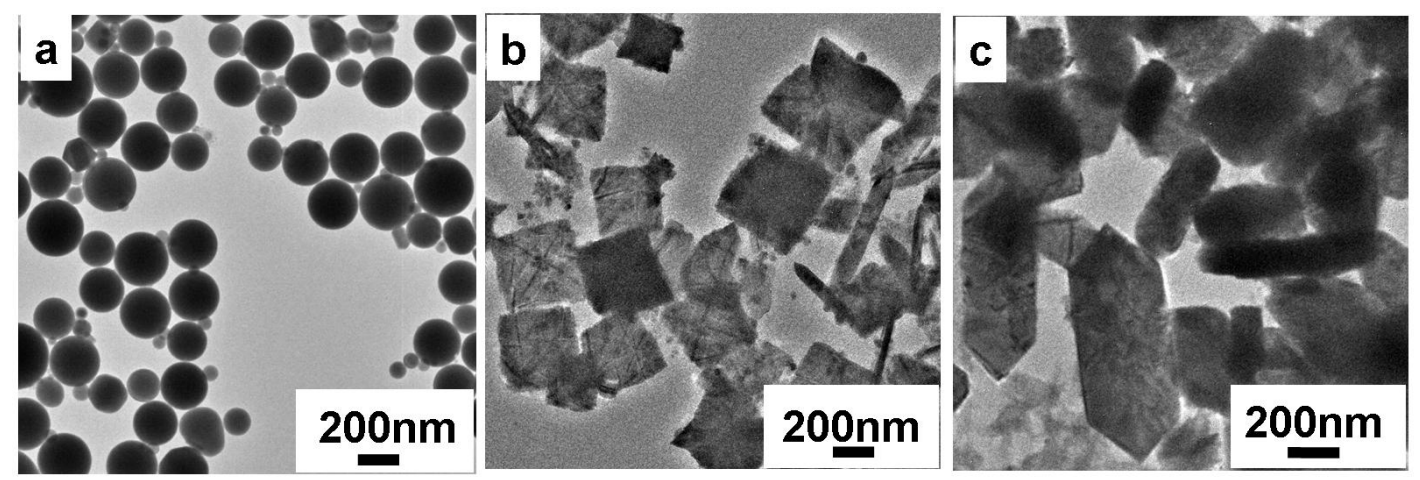

Fig. 2.2 TEM images of (a) tin spheres (b) tin monosulphide square plates and (c) tin monosulphide elongated hexagons.

\section{Particle size determination}

Many methods have been developed to measure particle size such as transmission electron microscopy (TEM), X-ray diffraction (XRD), extended X-ray adsorption fine structure (EXAFS), probe molecule absorption, optical absorption and small-angle neutron scattering (SANS). Each techniques has advantages and disadvantages but only TEM allows the direct visualization of nanoparticles at scales approaching a single atom. ${ }^{2}$ The discussion below is based on the work of Pyrz and Buttrey. ${ }^{2}$

Image analysis Once images have been acquired, the methods for image processing and size distribution must be chosen. Generally, size distribution is expressed through a Gaussian function, characterized by the mean and the standard deviation. Two significant factors that contribute to the uncertainty in both the mean and the standard deviation are pixel resolution and the bias associated with the determination of the particle edge. Image processing approaches can be classified as manual, software-assisted, or fully automated. The advantage of the manual and assisted methods is that each particle is scrutinized and measured, the signal-to-noise requirement is less stringent and analysis is straight forward. Whereas, the drawbacks include user biases and limited sampling statistics because only a few hundred nanoparticles can be 
measured within a reasonable time period. Alternatively, automated software package offer a variety of thresholding algorithms designed to locate and measure particles. Despite being automated, these routines also suffer from user bias because the user sets the criteria for discriminating the particle edge from the background. Accurate particle edge determination requires significant pixel intensity difference at the particles-substrate boundary. For nanoparticles with size less than $5 \mathrm{~nm}$ on a carbon film, simple thresholding routines that use only the intensity histogram are inadequate. Hence, improper application of these counting routines may result in significant over- or underestimation of particle size.

In this research, samples were prepared for TEM by one of two methods: (1) adding a drop of dilute solution of nanoparticle suspension to a copper grid with a layer of strong carbon film or (2) adding a drop of dilute solution of nanowire suspension to a holey carbon film on copper grid. Sonication was used to disperse the nanoparticles or nanowires prior to sampling.

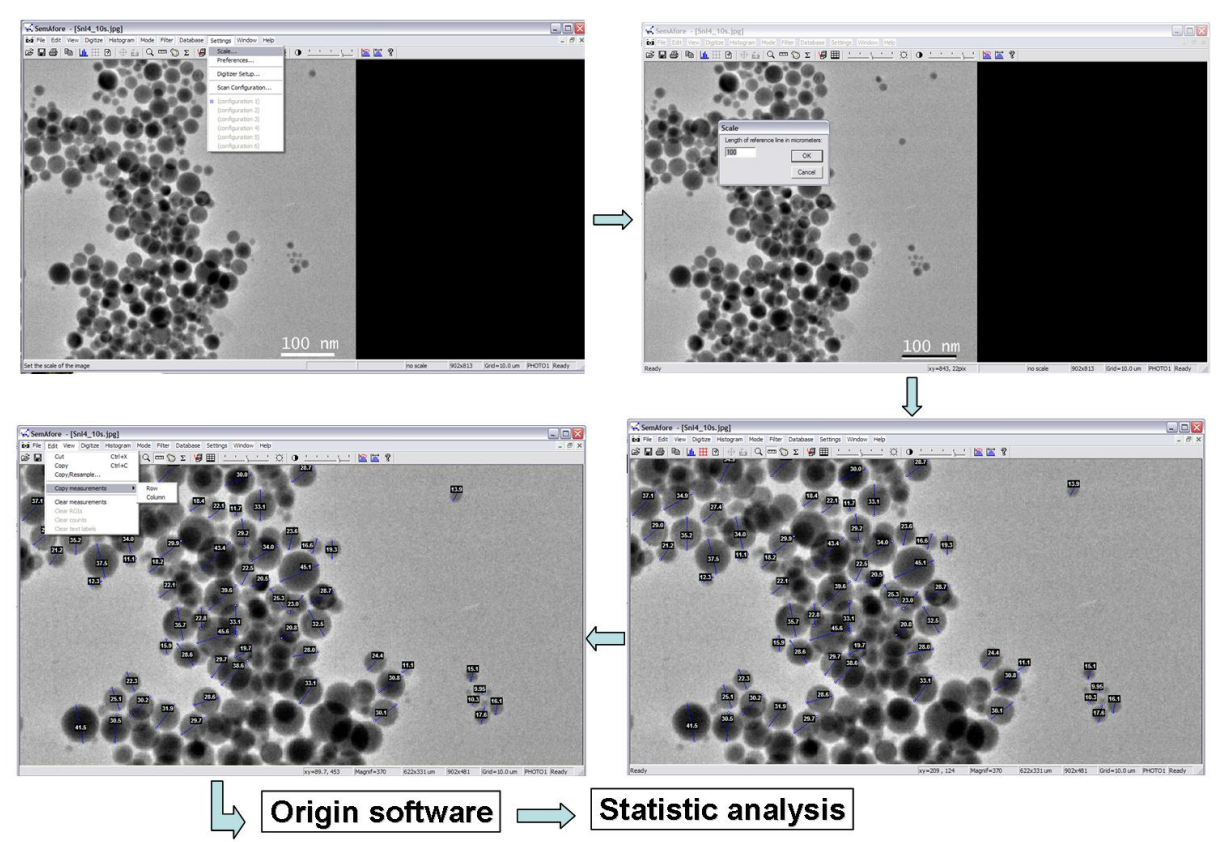

Scheme 2.2 Nanoparticles size distribution analysis. 
The size distribution of as-prepared nanoparticles was analyzed by using image analysis package: SemAfore Digitizer (Jeol), which offers an array of simple line-measurement tool. As shown in Scheme 2.2, the size of particles can be measured by using the internally calibrated scale bar displayed by the instrument's digital camera. The particles were measured manually. More than 100 nanoparticles or nanowires were measured for each sample. After the measurement, all the data were collected and transferred to Origin software to do the statistic analysis.

\section{Topographical Characterization}

Surface and interface features of objects can be investigated by using TEM. Fig. 2.3a shows a HRTEM image of a single SnGe nanowire (inset: LRTEM image of the nanowire), with some nanoparticles observed on the surface of the nanowire. Further investigation of the particles at higher magnification show the particles to be amorphous (Fig. 2.3b).
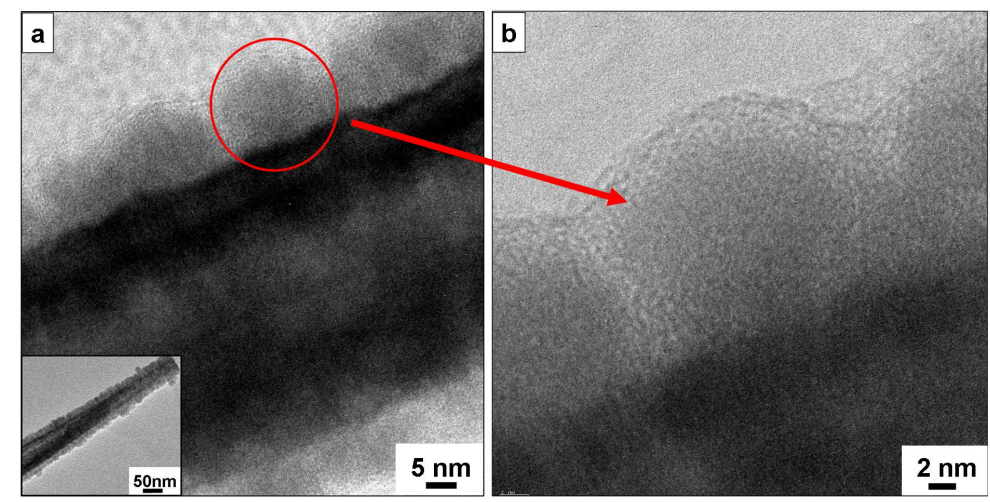

Fig. 2.3 Example of surface features investigated by using TEM. (a) HRTEM image of a single SnGe nanowire. (Inset: a LRTEM image of the nanowire.) (b) The corresponding HRTEM image of the red circle mark in image (a).

The structure information of an interface can also be elucidated by using TEM. A HRTEM image of the interface between the seed nanoparticle and nanowire is shown in Fig. 2.4. An abrupt structural change in the lattice, from seed 
nanoparticle to nanowire, is visible due to compositional and structural difference (discussion further in Chapter 4)

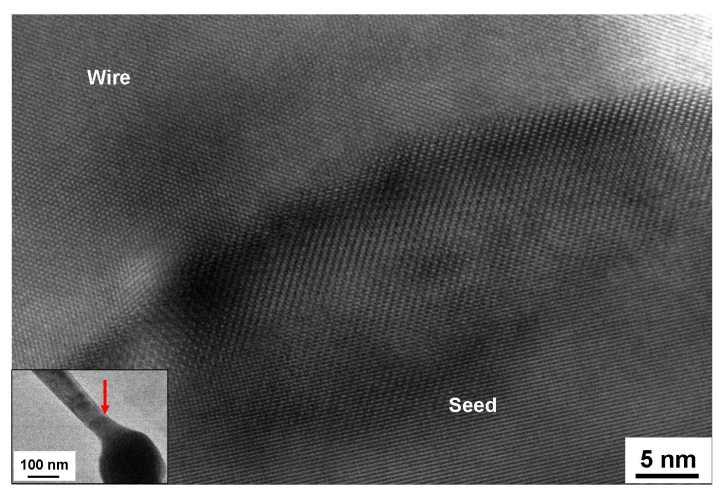

Fig. 2.4 HRTEM image showing interface between the Sn seeds nanoparticle and SnGe nanowire. (Inset: a LRTEM image of the nanowire, red arrow indicating the interface)

\section{Crystal defect observation}

High resolution TEM image affords the possibility of observing the packing of atoms in a crystal lattice when the crystal is perfectly focused and on zone (a zone is a set of planes, all of which are parallel to the direction of the electron beam in this case $\left.{ }^{3,4}\right)$. Fig. 2.5 shows a Bi seeded Ge nanowire with a twinning packing defect. The twin boundary (labeled with green line in Fig. 2.5) has a mirror symmetry.

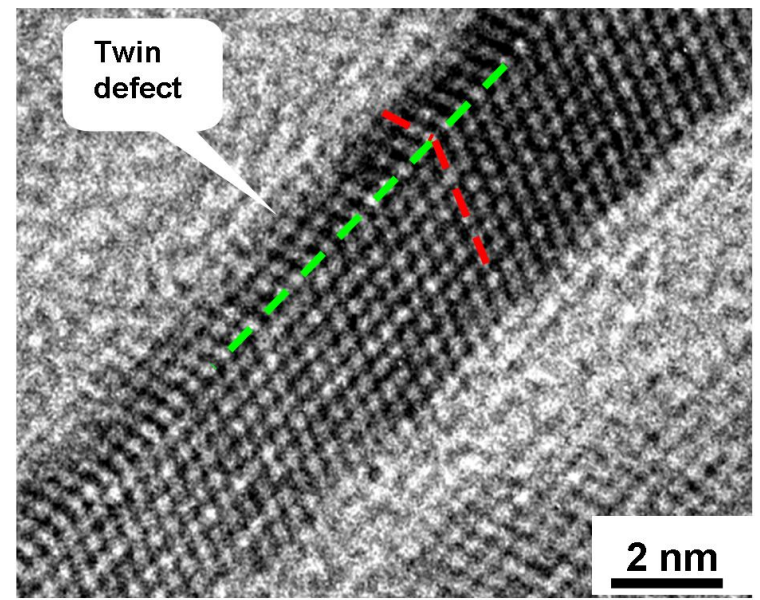

Fig. 2.5 Atomic resolution micrograph showing twin defect in a Bi seeded Ge nanowire. Red line showing twin planes; Green line showing a twin boundary 


\section{Fourier-transform Analysis}

The fast Fourier transform (FFT) pattern taken along the area (labeled in Fig. 2.6a) of the high-resolution TEM image of a Sn nanorod exhibits six sharp spots. An electron diffraction pattern (Fig. 2.6d) was simulated using CaRine crystallography 3.1 software. The simulated pattern matches the FFT pattern indicating that the nanorod is with [100] zone axis. The growth direction of the rod is determined as [0̄1].
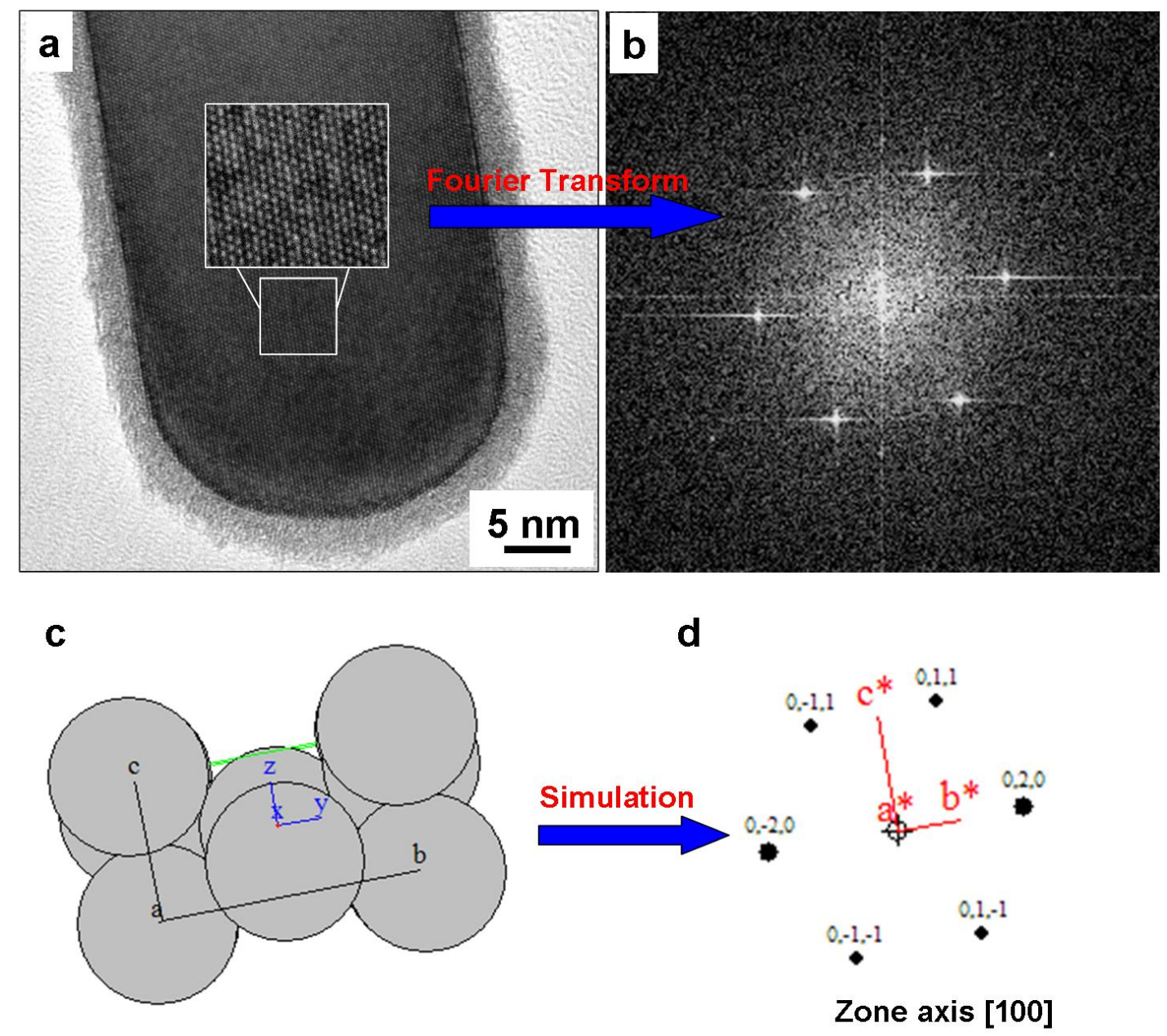

Fig. 2.6 HRTEM image of a Sn nanorod (inset: magnified image corresponding to the square area) (a) and corresponding FFT pattern(b); The unit cell of face-centered tetragonal Sn taken along the [100] zone axis (c) and the simulated diffraction pattern (d). 


\subsubsection{Characterization Using Electron Diffraction Pattern}

Selected Area Electron Diffraction (SAED) patterns can provide crystal structure information about the nanomaterials. The key advantages of SAED over powder X-ray diffraction are: (1) it is possible to obtain diffraction pattern from an individual sub-micron crystal. (2) It is possible to do the crystal structure measurement for extremely small nanoparticles (e.g. size range about 3-5 $\mathrm{nm})$. These small particles typically produce Powder XRD patterns with broad diffraction peaks which can not be indexed.

In this thesis, SAED pattern are commonly used to identify the crystal structure and examine crystal defects of the synthesized nanowires and nanoparticles. The pattern of sharp spots (Fig. 2.7a) or sharp rings (Fig. 2.8) contains information about the distance between atoms and hence can be used to determine the arrangement of the atoms. The d-spacings of SAED pattern were calibrated from those of gold standard.
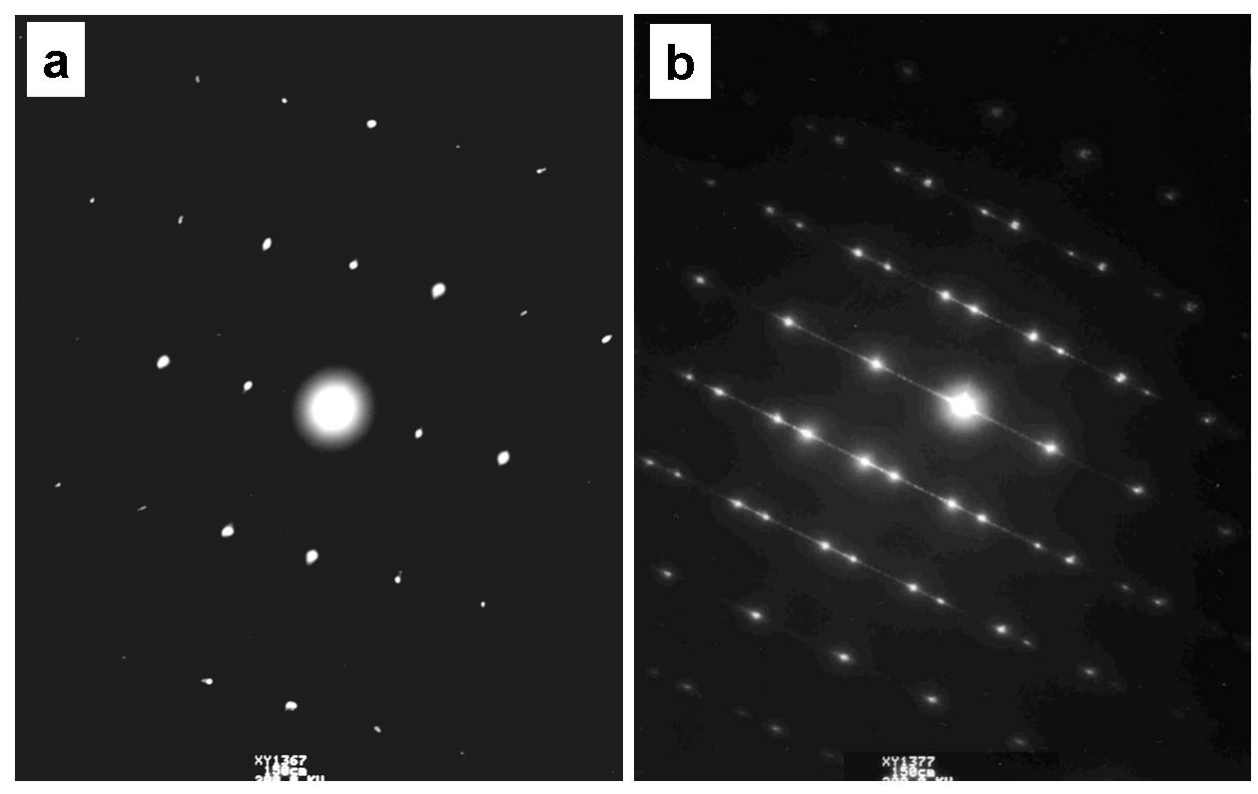

Fig. 2.7 Examples of SAED patterns for (a) a single crystalline SnGe nanowire (b)a twinned SnGe nanowire 


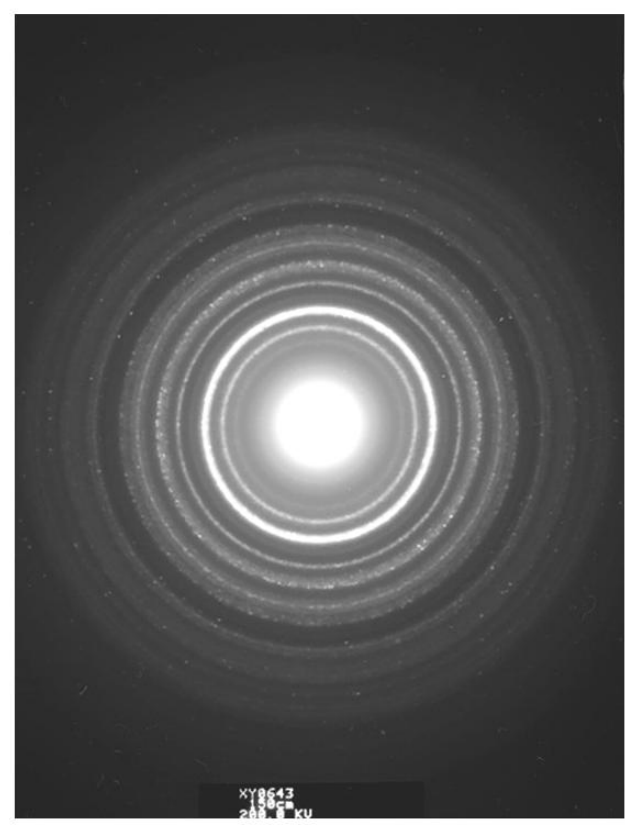

Fig. 2.8 orthorhombic phase SnS nanoparticle with an average diameter around $3 \mathrm{~nm}$.

\section{SAED Pattern for Nanowires Study}

Fig. 2.7 shows a typical electron diffraction patterns obtained from individual nanowires. A series of sharp spots are detected in the patterns. Indexing the diffraction pattern as shown in Fig. 2.7a, reveals that the SnGe nanowire is single crystalline, with a cubic structure. The growth direction of the nanowire can be resolved by correlating the indexed diffraction pattern with its corresponding TEM image. Furthermore, the presence of two series of diffraction spots in Fig. 2.7b demonstrates the formation of twin crystal in the selected area of the SnGe nanowire. This kind of examination can not be achieved by powder X-ray diffraction technique.

\section{SAED Pattern for Nanoparticles Study}

Fig. 2.8 shows an electron diffraction pattern obtained from SnS nanoparticles with an average size around $3 \mathrm{~nm}$. The pattern consists of multiple sharp bright rings which reveals the good crystalline nature of the nanoparticles. The 
orthorhombic structure of the nanoparticles can be indexed from the diffraction pattern. In contrast, the XRD pattern (Fig. 2.9) obtained from the same sample shows three unusually broad diffraction peaks which suggests that the size of nanoparticles could be very small but offer no information as to the SnS crystal structure.

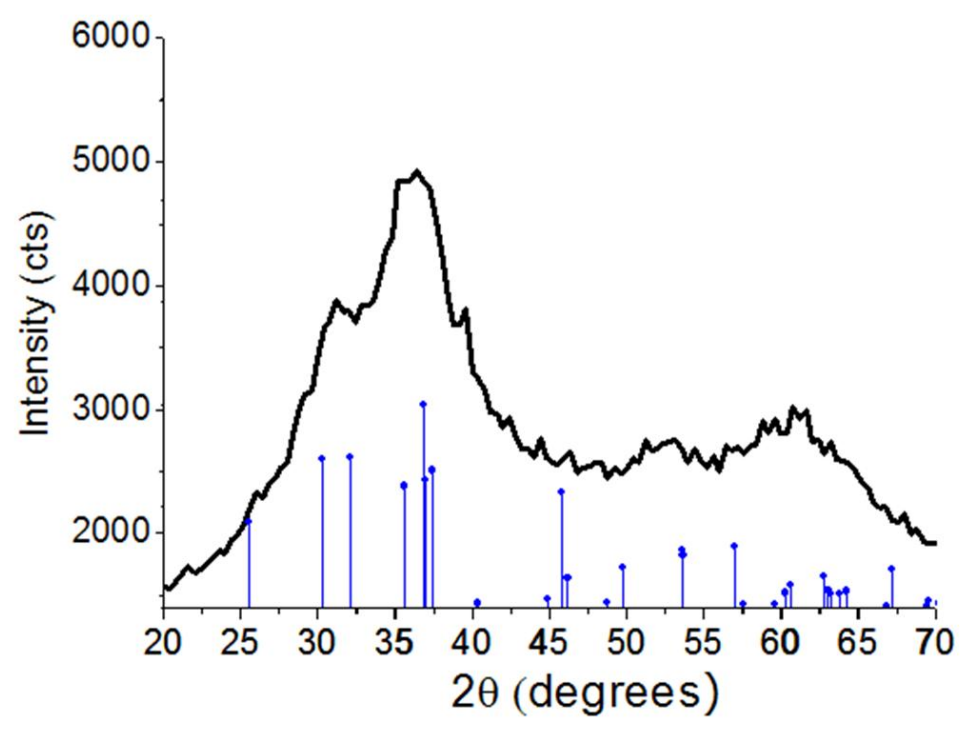

Fig. 2.9 XRD pattern (Co radiation) for the same nanoparticle sample. The expected positions and intensities for peaks corresponding to orthorhombic SnS (ICDD 01-071-3679) are marked by lines labeled with "•".

\subsection{Scanning Electron Microscopy}

Scanning Electron Microscopy (SEM) is another powerful type of electron microscopy that is widely used in material science. It has a large depth of field (i.e., the amount of sample that can be in sharp focus at one time) and can image the sample in three-dimensions which can not be achieved by using TEM. It can provide topographical, morphological and elemental information of a sample with a wide range of magnification (25x-500,000x). A JEOL 6500F Field-emission SEM of was employed to examine the morphology of the samples. 
A photograph of a high resolution SEM machine is shown in Fig. 2.10a along with a schematic diagram in Fig. 2.10b. A stream of electrons, produced by an electron source such as a: tungsten filament, tungsten field-emission tip or a lanthanum hexaboride $\left(\mathrm{LaB}_{6}\right)$ single crystal, is accelerated towards the specimen using a positive electrical potential. The stream is condensed and focused using metal aperture and magnetic lens (including two condenser lenses and one optical lens) into a focused fine spot on the specimen. The first two lenses (Fig. 2.10b) are condenser lenses as which are used to control the beam current and the final size on the specimen (spot size). The third lens is used to focus the electron beam on the surface of the specimen.
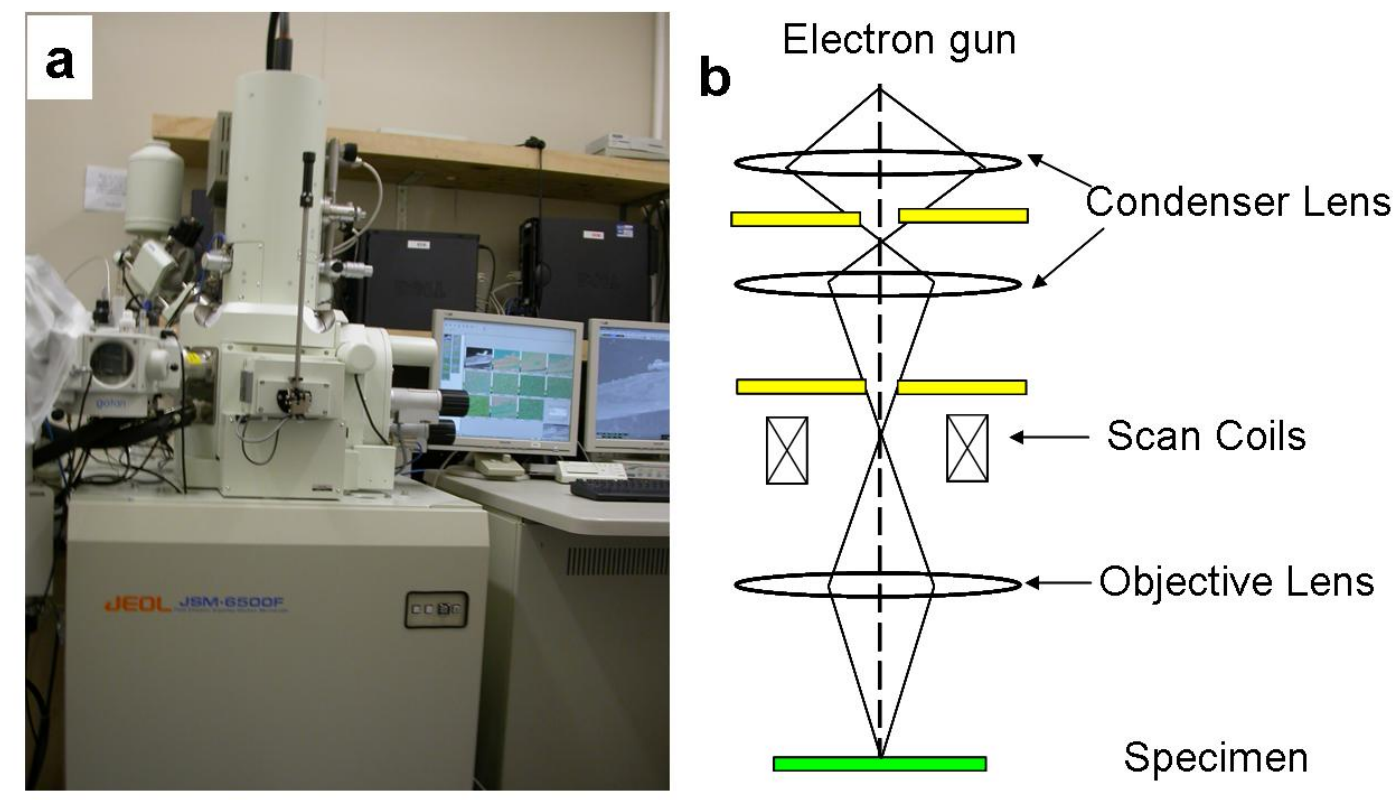

Fig. 2.10 (a) an high resolution SEM image; (b) Schematic representation of the electron beam.

The incident electrons, which carry significant amounts of kinetic energy, strike the sample and produce several different signals (Scheme 2.3). ${ }^{5,6}$ The major signals include secondary electrons, backscattered electrons and X-rays. Secondary electrons and backscattered electrons are commonly used for imaging samples. X-rays are used for elemental analysis or chemical characterization 
of a sample which will be described in detail in the section 2.3 .

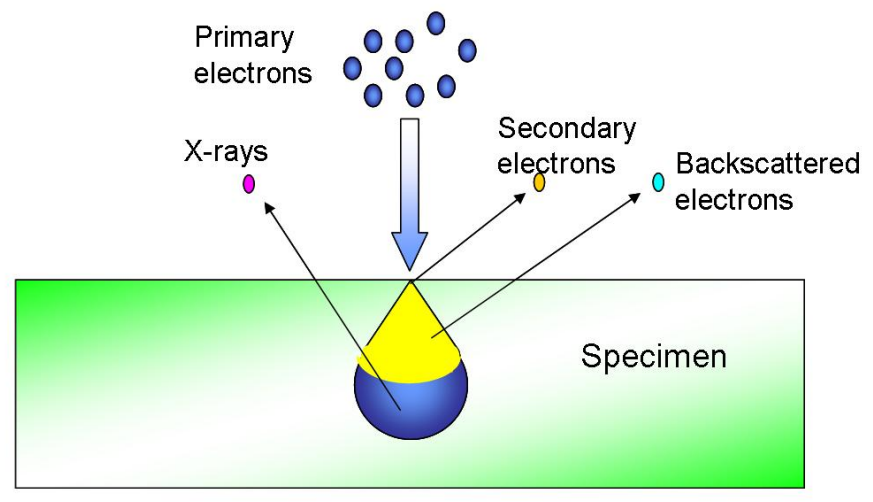

Scheme 2.3 Schematic of the interaction between incident electrons and specimen. ${ }^{6}$

Secondary electrons are created by the inelastic interactions between incident electrons and weakly bound conduction-band electrons in the atoms of the sample. Secondary electrons possess very small kinetic energy $(5 \mathrm{eV})$. Hence, the average distance that a secondary electron travels in the solid is less than $2 \mathrm{~nm}$. Because the secondary electron signal used in SEM is derived from secondary electrons that escape into the vacuum, the secondary electron image indicate the morphology and topography of the specimen.
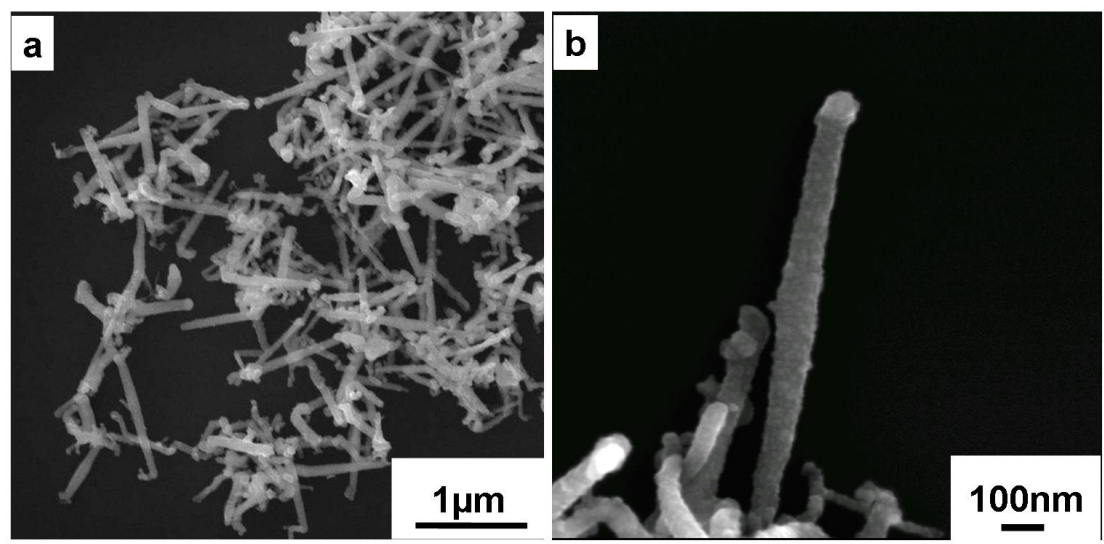

Fig. 2.11 Typical secondary electron images of SnGe nanowires: (a) a low magnification image indicate the diameter and length of the nanowires, (b) a high magnification image revealed the surface topography of the nanowires

Fig. 2.11 shows typical secondary electron images of SnGe nanowires. The 
length and diameter of an individual nanowire can be measured using a computer software package called SemAfore. The rough surface and tapped end of the nanowires can also be observed.

Backscattered electrons are generated by elastic interaction (deflecting angle $>90^{\circ}$ ) between incident electrons and the nucleus of the atoms in the sample. High energy backscattered electrons can penetrate deeper than secondary electrons in the solid sample. The penetration depth is strongly dependent on the specimen's atomic number (Z), decreasing with increasing Z (Scheme 2.4). For example, an electron with the energy of $1 \mathrm{keV}$ electron can penetrate about 50 $\mathrm{nm}$ into carbon but only $10 \mathrm{~nm}$ into gold. ${ }^{7}$

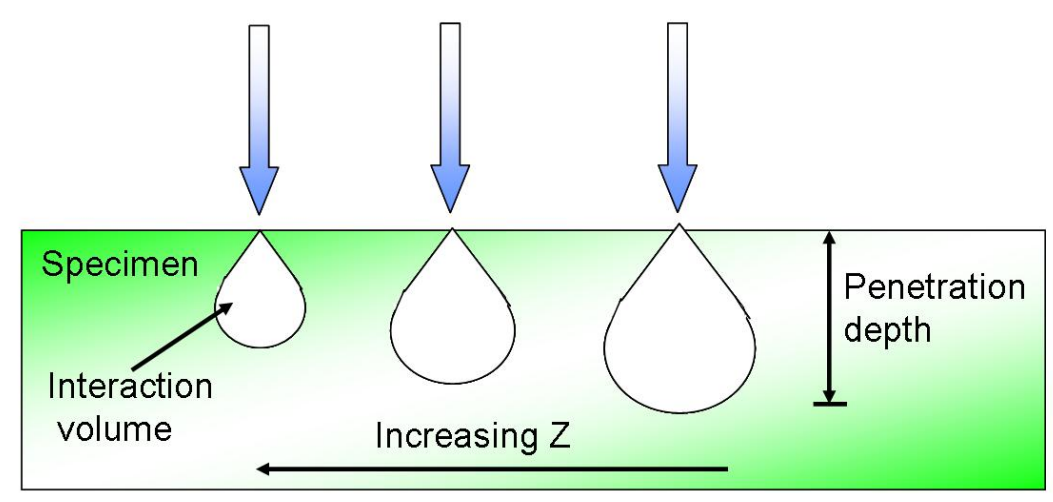

Scheme 2.4 Schematic showing dependence of the interaction volume and penetration depth as a function of atomic number $\mathrm{Z}$ of the incident (primary) electrons. ${ }^{7}$

The contrast mechanism of backscattered electrons relates to the dependence of the backscattering coefficient $\eta$ (the fraction of primary electrons that escape as backscattered electrons) on the mean atomic number: a region with higher atomic number elements appears brighter than lower atomic number elements in the backscattered electrons image. ${ }^{7}$ Fig. 2.12 shows a pair of secondary electron (SEI) and backscattered electron (BEI) images of same $\mathrm{Sn}_{1-x} \mathrm{Ge}_{x}$ nanowire sample. A comparison of the SEI and BEI images reveals the ability of BEI 
to differentiate between the $S n$ seed nanoparticles (atomic number $Z=50$ for $\mathrm{Sn}$ ) and Ge-rich $\mathrm{Sn}_{1-x} \mathrm{Ge}_{x}$ nanowires (atomic number $\mathrm{Z}=32$ for $\mathrm{Ge}$ ) based on contrast.
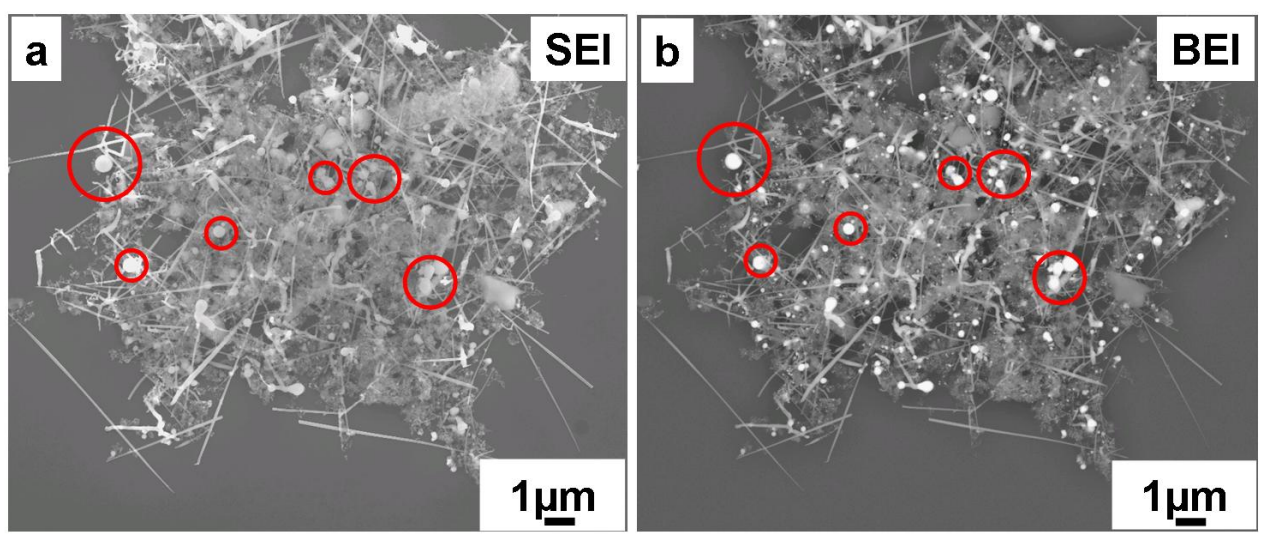

Fig. 2.12 (a) Secondary electron image (SEI) and (b) backscattered electron image (BEI) of a nanowire sample. Examples of Sn seed particles are shown in red circles.

\subsection{Energy Dispersive X-ray Spectrometry}

Energy Dispersive X-ray Spectrometry (EDX) is a powerful analytical technique performed in conjunction with a TEM (EDX detector: EX-23003 Bu) or SEM (EDX detector: EX-23000 Bu). TEM/EDX is often used to obtain the composition information of a thin sample at nano scale, while SEM/EDX is performed on micrometer scale samples. EDX measures the energy of X-rays that are produced by inelastic collisions of the incident electrons with electrons in discrete orbitals (shells) of atoms in the sample. As a lower energy (usually K-shell) electron was knocked out of the atom during the secondary electron process, an inner shell then has a vacancy. A high energy (outer shell) electron drops into the vacancy and releases the excess energy as an X-ray photon. Since each element has its own unique set of energy levels, the emitted photons are indicative of the element that produced them. 
The generated X-rays are collected by the EDX detector where the energy of the X-ray is determined. The number of X-rays are counted for each particular energy and displayed on a graph of counts vs. energy. Then based on an unique energy signature for each element, it is possible to determine the element composition of the sample. All elements with atomic number greater than Beryllium (Be) can be detected and quantitative analysis is possible.

EDX analysis can be generally divided into qualitative and quantitative analysis. Qualitative analysis includes survey analysis, EDX maps and line-scan profiles. Quantitative analysis is used to determine the chemical composition at one or more points of sample.

\subsubsection{Qualitative Analysis}

\section{Survey analysis}

Survey analysis is often used in the characterization of the composition of an unknown sample. It can quickly determine the elements which are present in the sample. Fig. 2.13 shows a typical EDX spectrum of an unknown sample. The EDX analysis indicates that the presence of element $\mathrm{Ge}, \mathrm{Sn}, \mathrm{Cu}, \mathrm{C}$, and $\mathrm{O}$. The signals of $\mathrm{C}$ and $\mathrm{Cu}$ are from carbon coated copper grid.

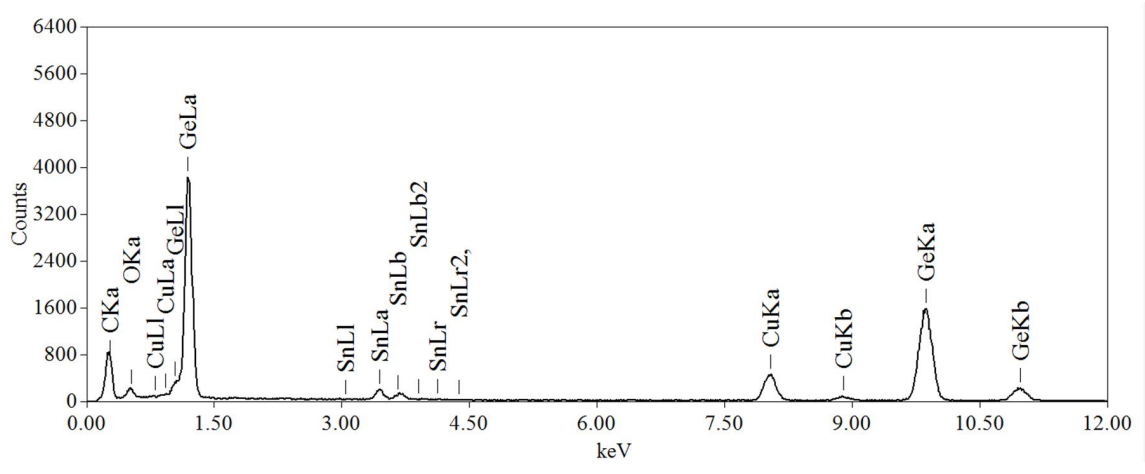

Fig. 2.13 A typical EDX spectrum of an unknown sample. 


\section{EDX mapping}

EDX mapping is widely used to identify areas of specific chemistry. It can determine the elemental distribution. During element mapping, one or more X-ray energies corresponding to elements of interest are collected as the SEM or STEM (TEM operating in scanning mode) scans the electron beam over a selected area. The X-ray data is synchronized with the SEM or STEM image and an elemental imageis generated.
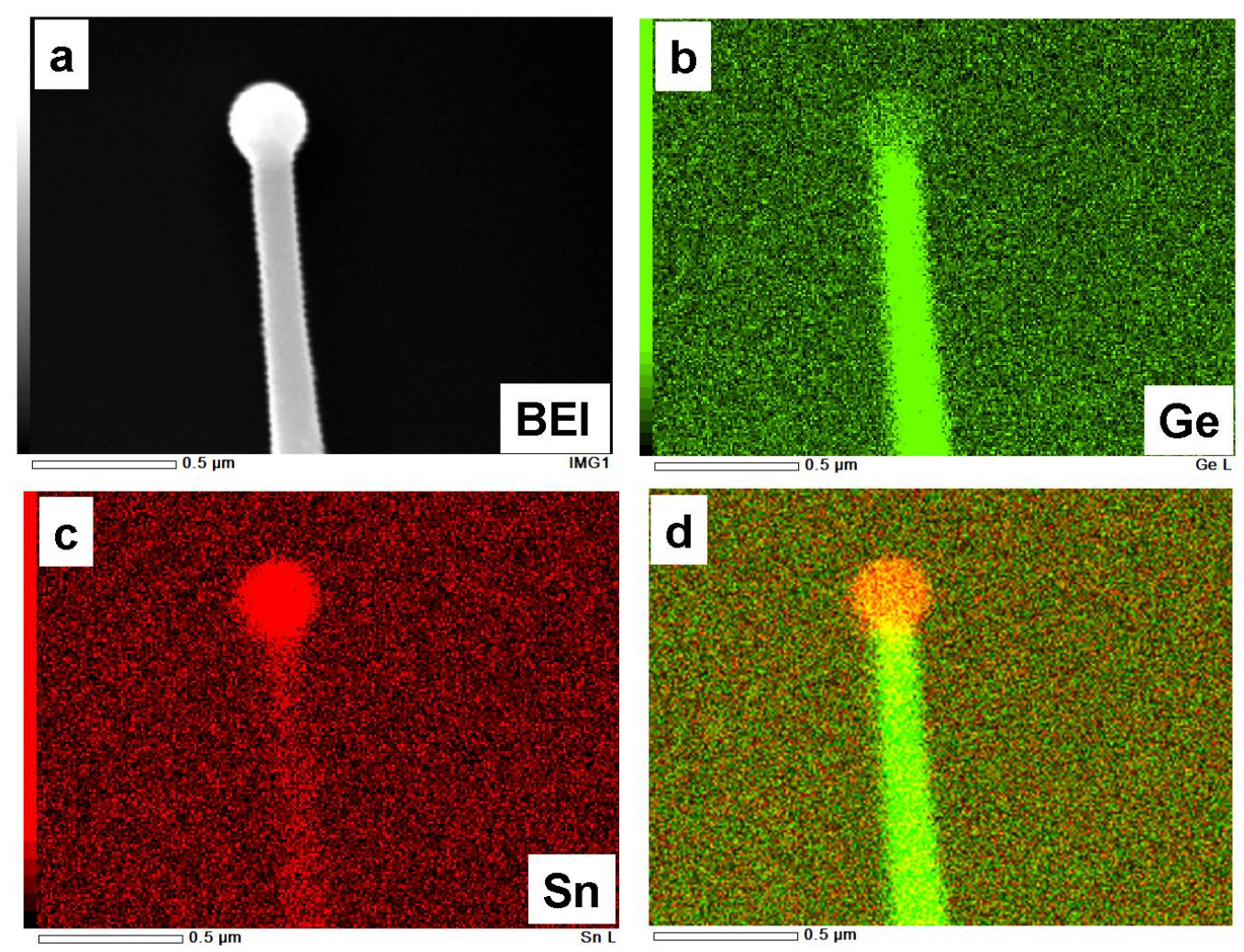

Fig. 2.14 SEM EDx mapping for a single nanowire: (a) a backscattered electron image (BEI); (b) EDX map of Ge; (c) EDX map for Sn; (d) EDX map overlays.

Fig. 2.14 shows a SEM backscattered electron image with EDX maps of Sn, Ge and map overlays of a single nanowire. The backscattered electron image clearly shows a white area at the seed segment and a grey area at the wire segment indicating the composition difference. EDX maps of elements indicate that there are regions which are rich (high intensity) in Ge (falsely coloured 
green) and rich (high intensity) in Sn (red) corresponding to the wire and seed respectively. The region where the elements Ge and Sn coexist exhibits yellow colour.

\section{Line-scan profiles}

Line-scan profiles are used to plot the abundance of an element with distance along a line. It is often used to examine trends at interfaces and concentration gradients within a sample. Fig. 2.15a is an EDX line scan profile along the scan line $(\mathrm{AB})$ depicted in the SEM image. The corresponding intensity profile in the line scan is shown in Fig. 2.15b. The results indicate the variation of the intensity of Ge and Sn along the nanowire.
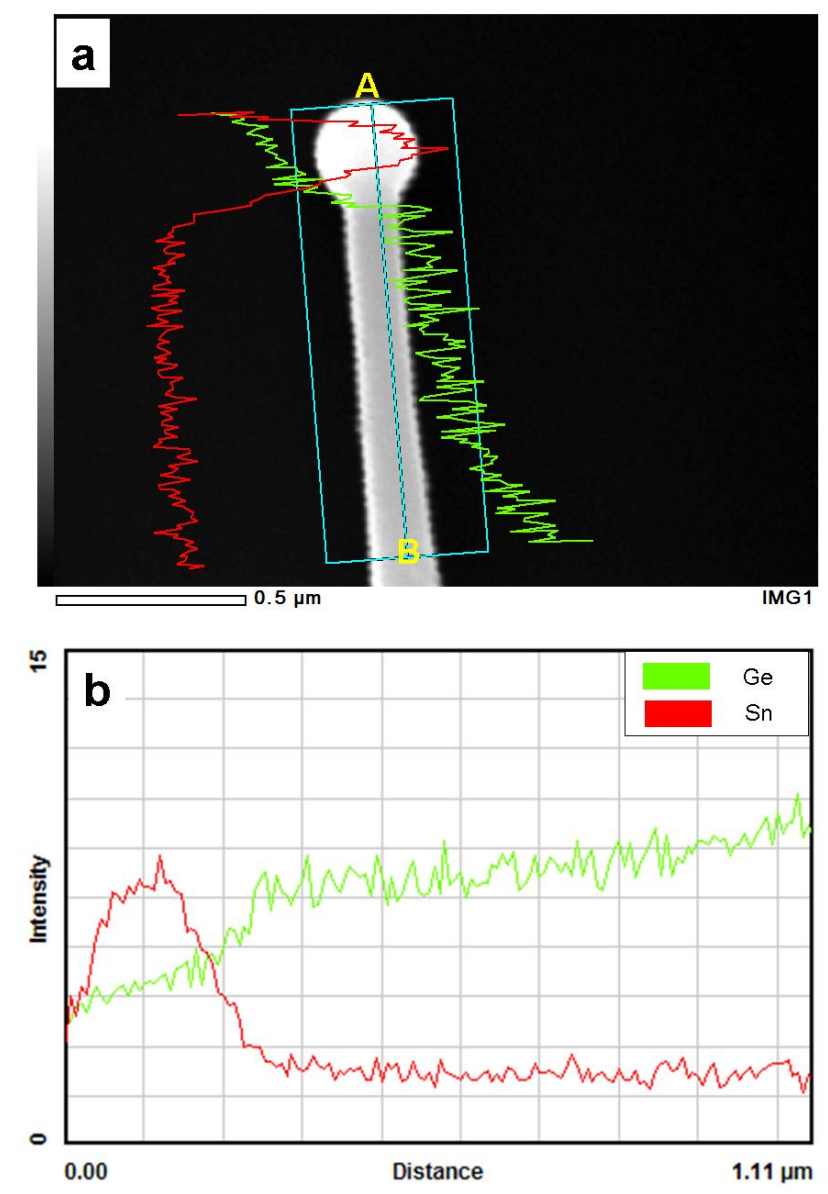

Fig. 2.15 (a) SEM EDX line scan profile along the scan line AB of a single nanowire; (b) Intensity profile in the line scan. 


\subsubsection{Quantitative Analysis}

Quantitative analysis involves determination of the abundance of the elements present in a sample. It provides chemical analysis of the field of view or spot analysis. Fig. 2.16 shows an example for spots analysis. The spots are labeled as 001 and 002 in the SEM image. The corresponding spectra are shown in Fig. 2.17. Quantitative analysis of the spectra yield the atomic ratio of Ge : Sn : Si in spot 001 and 002, respectively.

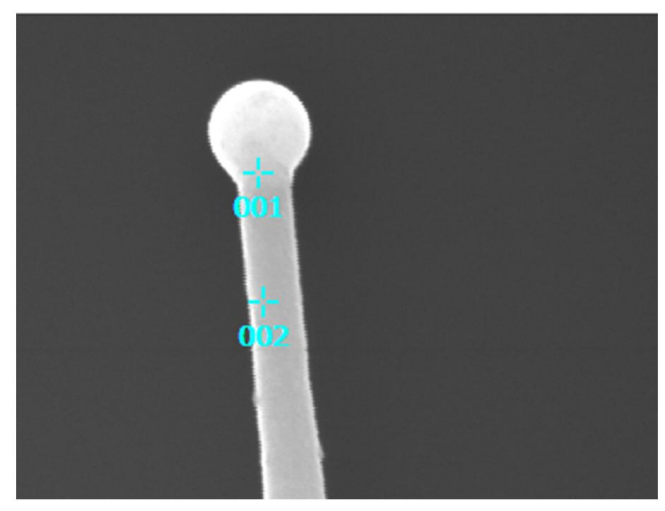

Fig. 2.16 A SEM image with two marked spot area.

a

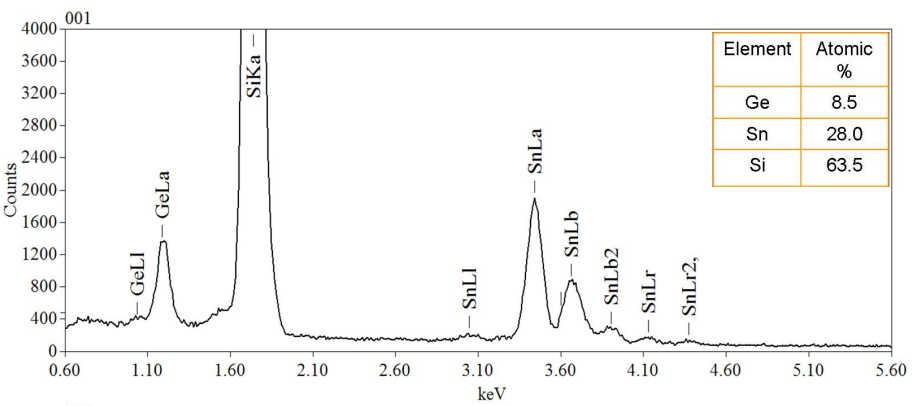

b

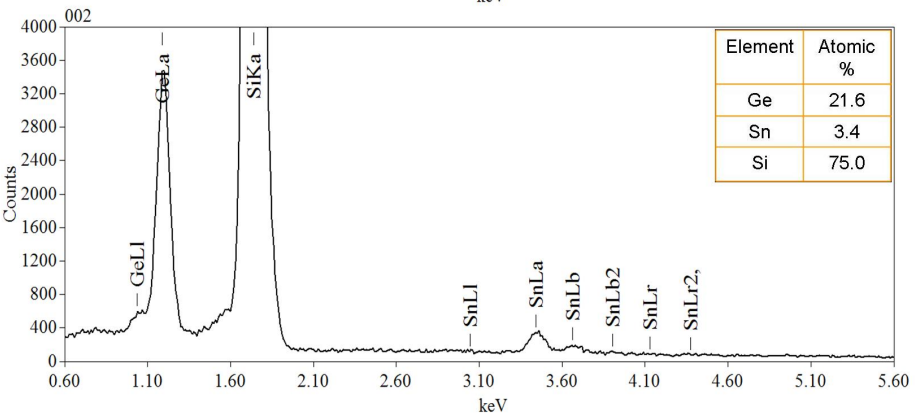

Fig. 2.17 The spectra recorded from spot 001 (a) and 002 (b). Inset : the result of atomic ratio of $\mathrm{Ge} / \mathrm{Sn} / \mathrm{Si}$. 


\subsection{Powder X-ray Diffraction}

Powder X-ray diffraction is an analytical technique primarily used for phase identification of crystalline materials and can determine: the dominant growth direction of one dimensional nanomaterials, the composition of alloys and crystallite size from line broadening.

Powder X-ray diffraction is based on constructive interference of monochromatic X-rays diffracted from the crystalline lattice of a sample. The interaction of the incident rays with the sample produces constructive interference when conditions satisfy "Bragg's Law" ( $\lambda=2 \mathrm{~d} \sin \theta)$ (Scheme 2.5). The diffracted $\mathrm{X}$-rays are collected and counted over a range of angles.

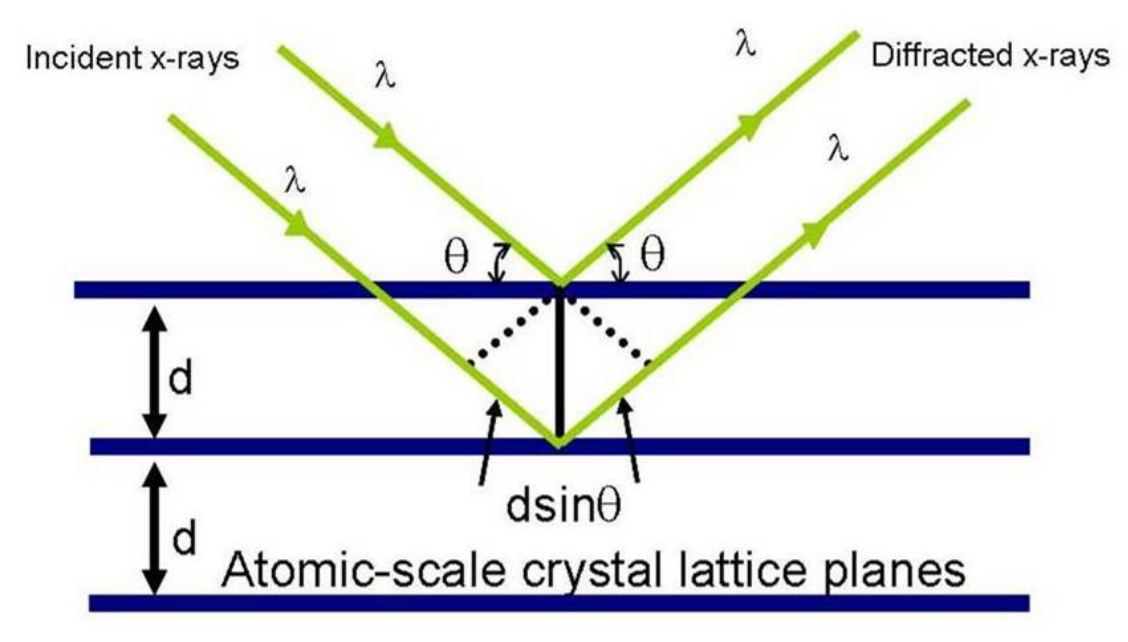

Scheme 2.5 Schematic diagram for determining Bragg's law.

\subsubsection{Phase Identification}

X-ray diffraction patterns can be used as fingerprints allowing identification of unknown crystalline phases. By comparing the positions and intensities of the peaks against a database of known crystalline materials, the target material can be identified. 
Moreover, the peak intensities reflect the total scattering from each plane in the phase's crystal structure. Hence, X-ray diffraction analysis of one dimensional nanowires can predict the dominant growth direction of the nanowires especially when taken in conjunction with Selected Area Electron Diffraction results.

Depending on the project, powder X-ray diffraction patterns were collected

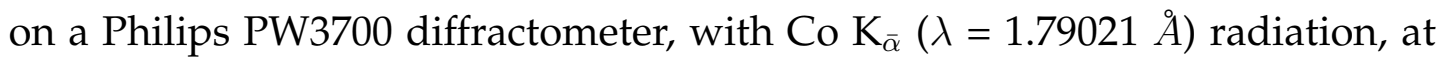
Industrial Research Limited (IRL) or a Philips PW3710 diffractometer, with Cu $\mathrm{K}_{\bar{\alpha}}(\lambda=1.54178 \AA)$ radiation, at Victoria University of Wellington (VUW). The compositions measured by EDX were compared to those determined by XRD.

\subsubsection{Determination of Alloy Composition}

From the composition dependence of the lattice parameter in semiconductor alloys, it is possible to determine of the molar fraction of the components assuming Vegard's law is valid. ${ }^{8}$ The expression of Vegard's law is:

$$
a(\text { mixture })=(1-x) a_{1}+x a_{2}
$$

where

$\mathrm{a}_{n}=$ Lattice parameters of the mixture and its components.

$\mathrm{x}=$ Mole fraction of the second component. 
The lattice parameters of a cubic cell $\left(\mathrm{a}=\mathrm{b}=\mathrm{c}, \alpha=90^{\circ}\right)$ can be calculated from X-ray diffraction data using the relation:

$$
a=\left[\left(h^{2}+k^{2}+l^{2}\right) d_{(h k l)}^{2}\right]^{0.5}
$$

where

$\mathrm{h}, \mathrm{k}, \mathrm{l}=$ Miller indices,

$d_{(h k l)}=$ inter-planar spacing between crystallographic planes.

In this thesis, X-ray diffraction patterns have been used to determine the composition of $\mathrm{Sn}_{x} \mathrm{Ge}_{1-x}$ nanowire samples. XRD measurements were made on drop-casted films of nanowires deposited on single crystal silicon substrate. The single crystal silicon also serves as an internal standard since peaks due to silicon do not overlap those from the $\mathrm{Sn}_{1-x} \mathrm{Ge}_{x}$ system (cubic phase) in the range of $2 \theta$ from 20 to 60 degrees.

The Philips X-ray diffractometer, PW-3710 (VUW) was used for these measurements.

\subsubsection{Determination of Crystallite Size}

For a polycrystalline sample consisting of a large number of crystallites, diffraction theory predicts that the breadth of the lines of the powder X-ray diffraction pattern are determined by both the mean crystallite size, distribution of crystallite size and the particular imperfection prevailing in the crystalline lattice. ${ }^{9}$ Therefore, analysis of the line profile should yield such information as the mean crystallite size and the nature and extent of lattice imperfections. ${ }^{9}$ Assuming that the nanoparticles are defect-free crystals, with spherical shape and of approximately uniform size,then line broadening is only caused by crystallite size L, which can be calculated by using the Scherrer equation: ${ }^{10}$ 


$$
L=\frac{k \lambda}{\beta \cos \theta}
$$

where

$\mathrm{L}=$ crystallite size,

$\mathrm{k}=$ shape factor (0.9 for spherical crystal morphology),

$\lambda=$ X-ray radiation wavelength $\left(\mathrm{Co} \mathrm{K}_{\alpha 1}=0.178897 \mathrm{~nm}\right)$,

$\theta=$ Bragg's diffraction angle (degree).

Additional instrument broadening, associated with the slit width, penetration in sample, imperfect monochromaticity and imperfect focusing is generally observed. ${ }^{11}$ In order to determine the instrumental contribution to line broadening, $\alpha$-alumina was used as an external standard in this study. The instrument broadening $\left(\beta_{I}\right)$ can be calculated using the relation. ${ }^{11}$

$$
\beta_{I}^{2}=U \tan ^{2} \theta+V \tan \theta+W
$$

Where $\mathrm{U}, \mathrm{V}$ and $\mathrm{W}$ are parameters of the function, determined using the alumina standard.

The line broadening, $(\beta)$, corrected from instrumental factors was estimated using the Warren formula.

$$
\beta=\left[\beta_{M}^{2}-\beta_{I}^{2}\right]^{0.5}
$$

Where

$\beta_{M}=$ measured integral breadth in radians of the sample,

$\beta_{I}=$ instrumental broadening in radians,

$\beta=$ corrected integral breadth in radians . 
Moreover, the mean crystallite size calculated by Scherrer equation is only reasonable for crystals which are smaller than several hundred nanometers and larger than $3 \mathrm{~nm}$ in size. The margin for error in this measurement is about $\pm 10 \%$. An estimation of the error was obtained from the error in the value of $\theta$ and $\beta$. The results from $\mathrm{X}$-ray techniques were compared to those obtained from direct observation of transmission electron microscopy.

In this thesis, X-ray diffraction pattern recorded in the range of $2 \theta$ from 20 to 70 degrees on Philips PW-3700 (IRL) have been used to determine the particle size in nanocrystalline tin telluride samples.

\subsection{Optical Properties Measurement}

\subsubsection{Ultraviolet-visible (UV-vis) spectrophotometer}

In the UV-vis absorption spectroscopy, the absorption intensity is scanned with wavelength. This is called absorption spectrum. This technique can be applied to any molecular species, organic and inorganic, in solution. Sometimes, the absorption spectrum shows peaks of absorption but in some cases it shows a continuous absorption graph. The absorption spectrum is due to electron transfer from a lower occupied electronic level (ground state) to a higher unoccupied electronic level (excited state). Therefore, absorption peaks represent the energy difference between these two levels. The concentration of an analyte in solution can be determined by measuring the absorbance at some wavelength and applying the Beer-Lambert Law:

$$
A=\alpha * b * c
$$


where

$\mathrm{A}=$ absorbance .

$\mathrm{a}=$ absorption coefficient.

$b=$ the path length.

$\mathrm{c}=$ the analyte concentration.

Quantum dots consist of many molecular units arranged in a crystalline lattice. The absorption spectrum for quantum dots appears as a series of overlapping peaks that get larger at shorter wavelengths. Owing to the discrete nature of electron energy levels in quantum dots, each peak corresponds to an energy transition between discrete electron-hole (exciton) energy levels. The quantum dots will absorb light that has a wavelength shorter than that of the first exciton peak, also referred to as the absorption onset. The wavelength of the first exciton peak is a function of the composition and size of the quantum dot. Smaller quantum dots result in a first exciton peak at shorter wavelengths.

A Varian Cary 50 double beam Bio UV-visible spectrophotometer was used for UV-vis absorption measurements. The instrument utilizes a xenon flash lamp as light source which offers many advantages over traditional UV-Vis spectrophotometer (using diode array as light source). The Xenon lamp is very intense which allows the instrument to use a beam splitter for simultaneous reference beam correction. In addition, the peaks will not shift as the scan speed changes. It has a broad wavelength range from $200 \mathrm{~nm}$ to $1000 \mathrm{~nm}$.

Samples were prepared as dilute suspensions in an appropriate solvent. Pure solvent was used for baseline calibration before the sample measurement start. 


\subsubsection{Fluorescence spectroscopy}

Fluorescence is emission light from singlet-excited states, in which the electron in the excited orbital is paired to the second electron in the ground -state orbital. Returning of the electron to the ground state is spin allowed and occurs rapidly by emission of a photon. Fluorescence spectral data are generally presented as emission spectra.

The peak emission wavelength of quantum dots is usually bell-shaped (Gaussian) and occurs at a slightly longer wavelength than the lowest energy exciton peak (the absorption onset). This energy separation is what is referred to as the Stoke's Shift. An interesting property of quantum dots is that the peak emission wavelength is independent of the wavelength of the excitation light, assuming that it is shorter than the wavelength of the absorption onset. The bandwidth of the emission spectra, denoted as the Full Width at Half Maximum (FWHM) stems from the temperature, natural spectral line width of the quantum dots, and the size distribution of the population of quantum dots within a solution or matrix material. Spectral emission broadening due to size distribution is known as inhomogeneous broadening and is the largest contributor to the FWHM. Narrower size distributions yield smaller FWHM. ${ }^{12}$

A Horiba Jobin Yvon Fluorolog-3 spectrofluorimeter (FluoroLog-3 ) was used for photoluminescence measurement. FluoroLog-3 instrument equipped with a $450 \mathrm{~W}$ xenon $\mathrm{CW}$ lamp as a light source. It has wide detection range from the UV to the IR. Measurements were made on dilute solution of nanoparticles dispersed in a solvent or films of nanoparticles deposited on glass substrates. 


\subsubsection{Diffuse reflectance spectrometer}

Diffuse reflectance relies upon the focused projection of the spectrometer beam into the sample where it is reflected, scattered and transmitted through the sample. The back reflected, diffusely scattered light (some of which is absorbed by the sample) is then collected by the accessory and directed to the detector optics. Only the part of the beam that is scattered within a sample and returned to the surface is considered to be diffuse reflection.

Diffuse reflectance spectroscopy is used to estimate band gap energies of semiconductor nanomaterials. ${ }^{13,14}$ A common way of extracting the band gap from diffuse reflectance spectra is to get the first derivative of reflectance with respect photon energy and find the maximum in the derivative spectra. ${ }^{15}$

Diffuse reflectance spectra were recorded on a High Accuracy Reference Spectrophotometer at IRL. ${ }^{16}$ The wavelength range from $250 \mathrm{~nm}$ to $2500 \mathrm{~nm}$. The reflectivity measurements were made on drop-cast films of nanoparticles deposited on glass substrates.

\subsubsection{Fourier Transform Infrared (FTIR) spectrometer}

Infrared spectroscopy reveals information about the vibrational states of a molecule. Intensity and spectral position of IR absorptions allow the identification of the structure of a molecule. It can be used to identify different functional groups such as $-\mathrm{OH}$ group, $-\mathrm{COOH}$ group, $-\mathrm{NH}_{2}$ group and $-\mathrm{CH}_{2}$ group.

\section{PerkinElmer FTIR spectrometer}

PerkinElmer spectrum One FTIR spectrometer with wavenumber range of 4000$400 \mathrm{~cm}^{-1}$ was used to determine the absorption of organic ligand on the nanopar- 
ticles surface. Pellets for FTIR measurement were prepared by mixing dried samples with spectroscopic grade $\mathrm{KBr}$ powder. Equal mass of $\mathrm{KBr}$ was used as a reference.

\section{Bomem DA8 FTIR spectrometer}

A vacuum Bomem DA8 FTIR spectrophotometer with calcium fluoride $\left(\mathrm{CaF}_{2}\right)$ beam splitter was used to record the near infrared absorbance spectra of the samples with a band gap in the IR region such as SnTe nanoparticles. The measurement was recorded from $8000 \mathrm{~cm}^{-1}$ to $2000 \mathrm{~cm}^{-1} . \mathrm{CaF}_{2}$ discs $(25 \mathrm{~mm}$ in diameter) were used to support sample films for the FTIR measurements. Sample solutions were drop cast on the $\mathrm{CaF}_{2}$ discs. A blank spectrum was measured from the bare $\mathrm{CaF}_{2}$-disc under identical measurement conditions. 


\section{REFERENCES}

[1] Buseck, P.; Cowley, J. M.; Eyring, L. High-resolution transmission electron microscopy and associated techniques; Oxford University Press, Inc., 1988.

[2] Pyrz, W. D.; Buttrey, D. J. Langmuir 2008, 24, 11350-11360.

[3] Brydson, R.; Castell, M.; Craighead, H. G.; Dunin-Borkowski, R.; Gai, P. L.; Hutchison, J.; Kirkland, A.; Midgley, P.; O’Brien, P.; Pennycook, S. J.; Smith, D. J. Nanocharacterisation; RSC Publishing, 2007.

[4] Tilley, R. J. D. Crystals and Crystal Structures; John Wiley \& Sons, Ltd, 2006.

[5] Vernon-Parry, K. D. III-Vs Review 2000, 13, 40-44.

[6] A Guide to Scanning Microscope Observation; JEOL Serving Advanced Technology.

[7] Egerton, R. F. Physical Principles of Electron Microscopy: An Introduction to TEM, SEM, and AEM; Springer, 2005; pp 125-140.

[8] Denton, A. R.; Ashcroft, N. W. Physical Review A 1991, 43, 3161-3164.

[9] Klug, H. P.; Alexander, L. E. X-Ray Diffraction Procedures for Polycrystalline and Amorphous Materials; Wiley, New York, 1974; pp 642-641.

[10] Patterson, A. L. Physical Review 1939, 56, 978-982.

[11] Caglioti, G.; Paoletti, A.; Ricci, F. Nuclear Instruments 1958, 3, 223 - 228.

[12] Lakowicz, J. R. Principles of fluorescence spectroscopy; Kluwer Academic Publishers, 1999.

[13] Murphy, A. Solar Energy Materials and Solar Cells 2007, 91, 1326-1337.

[14] Wendlandt, W. W.; Hecht, H. G. Reflectance spectroscopy; Interscience Publishers, 1966. 
[15] Marotti, R.; Giorgi, P.; Machado, G.; Dalchiele, E. Solar Energy Materials and Solar Cells 2006, 90, 2356-2361.

[16] Hamlin, J. Diffuse reflectance spectra were record on a High Accuracy Reference Spectrophotometer at measurement standards laboratory of New Zealand (MSL)/ IRL, the measurements were done by John Hamlin; Technical Report, 2009. 


\section{Chapter 3}

\section{Synthesis of Tin nanoparticles}

\section{Contents}

3.1 Introduction . . . . . . . . . . . . 65

3.2 Tin nanoparticles Synthesis and Characterization . . . . . . 68

3.3 Summary $\ldots \ldots \ldots \ldots \ldots \ldots \ldots$

\subsection{Introduction}

This chapter is focused on the solution phase synthesis of tin nanoparticles. The synthetic approach described here involves the chemical reduction of inexpensive tin halide with sodium borohydride.

It is known that metallic tin (bulk) has a melting point of $232^{\circ} \mathrm{C}$ which is much lower than that of many other metals, such as silver $\left(961^{\circ} \mathrm{C}\right)$, gold $\left(1065^{\circ} \mathrm{C}\right)$ and nickel $\left(1453^{\circ} \mathrm{C}\right) .{ }^{1}$ The melting point of pure metals can be dramatically decreased by reducing their size to a nanometer scale. ${ }^{2-4}$ Over the past decades, the particle size dependent melting point depression for nanosized metallic tin has been extensively studied both in theory ${ }^{5,6}$ and experiment. ${ }^{7,8}$ As shown in Fig. 3.1, Jiang et al. reported that the melting points of tin nanoparticles of 85 
$\mathrm{nm}, 52 \mathrm{~nm}$ and $26 \mathrm{~nm}$ of tin nanoparticles are $231.8^{\circ} \mathrm{C}, 228^{\circ} \mathrm{C}$ and $214.9^{\circ} \mathrm{C}$, respectively. It is clearly demonstrated that the melting point of tin nanoparticles decreases as the particle size decreases. ${ }^{8}$ The observed melting point depression of tin nanoparticles is due to their high surface-to-volume ratio and their surface energy which substantially effects the interior "bulk" properties of the material. ${ }^{5}$

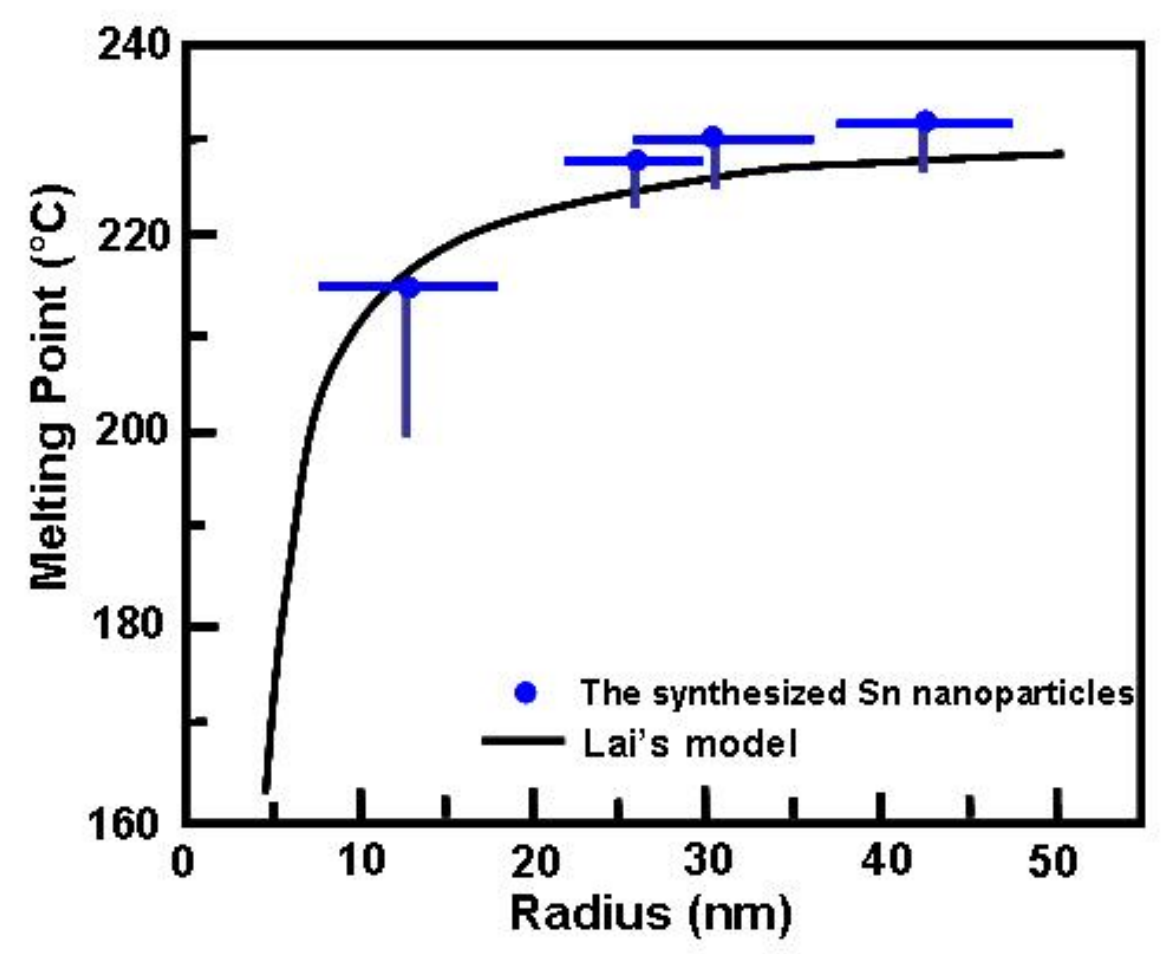

Fig. 3.1 Size dependence of the melting points of tin nanoparticles (Y error bars stand for the melting temperature from the onset point to the peak point of DSC curves). The solid line is calculated from Eq.(1) by Lai et al. ${ }^{8}$

After the first introduction of using low melting point indium metal $\left(156.4^{\circ} \mathrm{C}\right)$ nanoparticle as a catalyst for the solution-liquid-solid (SLS) growth of semiconductor InP nanowires by Buhro's group, ${ }^{9}$ the other low melting point metal nanoparticles such as bismuth and tin have attracted more attention. High yield germanium nanowires synthesized using bismuth nanoparticles as a cat- 
alyst has been reported by Korgel and coworker. ${ }^{10}$ The use of tin nanoparticles as a catalyst for the growth of semiconductor nanowires is still relatively new. Current research in the synthesis of size controlled tin nanoparticles is motivated by using these low melting point nanoparticles for the growth of tin germanium nanowires which will be described in detail in Chapter 4 .

Generally, the synthesis of tin nanoparticles can be achieved through either a "top down" approach or a "bottom up" approach ("top down" and "bottom up" approaches have been explained in Chapter 1). To be more specific, "top down" approach for preparation of tin nanoparticles includes ultrasound dispersion ${ }^{11}$ and solution dispersion. ${ }^{12}$ The as-synthesized tin nanoparticles are nearly spherical in shape with a large size distribution. The particles were large with a range from $50 \mathrm{~nm}$ to $3500 \mathrm{~nm} .{ }^{11}$ These methods are commonly used to prepare big tin nanoparticles (size $>50 \mathrm{~nm}$ ) in large scale. To prepare tin nanoparticles smaller than $50 \mathrm{~nm}$, remains a big challenge. In contrast, a "bottom up" approach such as thermal decomposition ${ }^{13}$ and chemical reduction can be used to prepare tin nanoparticles smaller than $50 \mathrm{~nm}$ by varying the reaction parameters. Bruno et al. reported a method of forming tin nanoparticles of less than $20 \mathrm{~nm}$ in size via thermal decomposition. ${ }^{13}$ The synthesis of Bruno and coworkers uses the air sensitive and expensive organometallic tin precursors $\left\{\left[\mathrm{Sn}\left(\mathrm{NMe}_{2}\right)_{2}\right]_{2}\right\} \cdot{ }^{13}$ Lavinia and coworkers prepared tin nanoparticles through a low temperature $\left(65^{\circ} \mathrm{C}\right)$ chemical reduction, where $\mathrm{SnCl}_{2}$ was reduced by $\mathrm{t}$-BuONa-activated $\mathrm{NaH}$ in THF for $30 \mathrm{~min}$. The average particle size of as-synthesized tin nanoparticles was around $40 \mathrm{~nm} .{ }^{14}$ However, these methods produce particles with a broad size distribution.

In order to produce nearly uniform nanoparticles, the distinct separation between the nucleation and growth stages is essential. ${ }^{15}$ The separation of nucle- 
ation and growth can be achieved by rapid injection of reagents into the hot solution, which raise the monomer concentration above the nucleation threshold ("rapid hot-injection" method"16), resulting in burst nucleation. The burst nucleation can be quickly quenched by the fast cooling of the reaction mixture and by the decrease supersaturation after nucleation burst. ${ }^{17}$ Hence, in the current research, the "rapid hot-injection" method is employed to produce nearly monodispersed tin nanoparticles of small size.

Several methods exist to prepare tin nanoparticles, with simplicity and affordability remaining to be a big challenge. Hence, the objectives of this research were: (1) to develop a facile and cheap synthesis approach for preparing tin nanoparticles; (2) to prepare nearly uniform tin nanoparticles and understand the effect of reaction parameters such as reaction temperature, concentration and surfactant on the size and size distribution of the as-synthesized nanoparticles.

\subsection{Tin nanoparticles Synthesis and Characteriza- tion}

In this series of experiments, tin nanoparticles have been synthesized via chemical reduction in the presence of a surfactant at high temperature $\left(150{ }^{\circ} \mathrm{C}\right.$ $300{ }^{\circ} \mathrm{C}$ ) for 1 minute. As shown in Scheme 3.1, the general procedure employed here can be referred to as a "rapid hot injection" method. ${ }^{16}$ This rapid hot injection method is similar to the conventional method except that reduction instead of decomposition of precursor in the synthesis system takes place. 


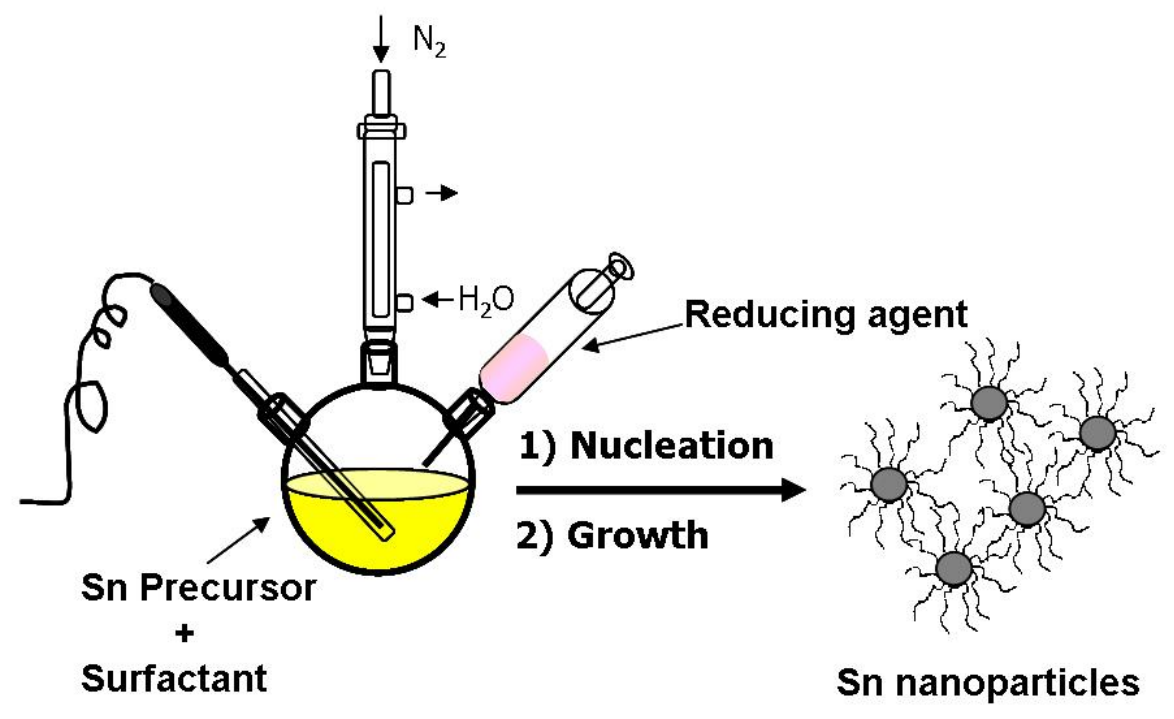

Scheme 3.1 Synthesis of capped tin nanoparticles from reduction of a tin precursor.

In the synthesis of tin nanoparticles, the tin halides including $\mathrm{Sn}(\mathrm{IV}) \mathrm{I}_{4}$ and $\mathrm{Sn}(\mathrm{II}) \mathrm{Cl}_{2}$ were selected as tin precursors. These precursors are of relatively low

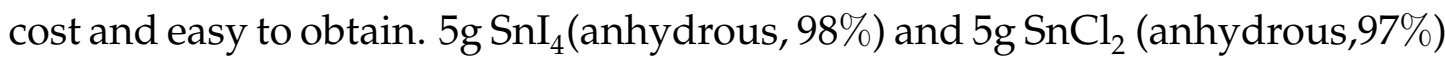
purchased from Sigma-Aldrich cost about NZ $\$ 267$, and NZ $\$ 77$, respectively. Furthermore, these tin precursors are easy to handle during the synthetic process which is more convenient over the other air sensitive organometallic tin precursor such as $\left\{\left[\mathrm{Sn}\left(\mathrm{NMe}_{2}\right)_{2}\right]_{2}\right\} .{ }^{13}$ The use of $\mathrm{Sn}(\mathrm{IV}) \mathrm{I}_{4}$ as a tin precursor has not been reported in the literature whereas $\mathrm{Sn}(\mathrm{II}) \mathrm{Cl}_{2}$ has been commonly used for the synthesis of tin nanoparticles. ${ }^{14}$ It is expected that $\mathrm{SnCl}_{2}$ can be easily reduced to $\mathrm{Sn}(0)$ compared to $\mathrm{SnI}_{4}$ as indicated by the following equations: ${ }^{18}$

$$
\begin{aligned}
S n^{4+}+2 e \rightarrow S n^{2+} & E^{\ominus}=0.15 V \\
S n^{2+}+2 e \rightarrow S n(0) & E^{\ominus}=-0.14 V
\end{aligned}
$$

Where $E^{\ominus}$ is the standard reduction potential. 
$\mathrm{NaBH}_{4}$, as one of the most common reducing agent, was used in the synthesis. It is a cheap (100g, $\mathrm{NZ} \$ 310)$, safe and easily handled reagent. It is soluble in some organic solvents such as, tetraglyme and triglyme, which is important for the injection of reducing agent into a hot tin precursor solution.

Trioctylamine (TOA) used in the synthesis plays dual roles. It is a solvent to enable tuning of the reaction temperature over a broad range (up to $300{ }^{\circ} \mathrm{C}$ ) and can also act as a surfactant to stabilize and control the tin nanoparticles.

In the following of experiments, the influence of the process variables (e.g., temperature, concentration, surfactant, precursor) on the average particle size have been investigated. Particle size distributions were estimated from TEM images by measuring the sizes of more than 100 nanoparticles per system.

\subsubsection{Experiment A: Reaction at $150{ }^{\circ} \mathrm{C}$}

Initially, tin nanoparticles were prepared via chemical reduction of $\mathrm{SnI}_{4}$ with $\mathrm{NaBH}_{4}$ in TOA. The reaction temperature was selected at $150{ }^{\circ} \mathrm{C}$ which is lower than the melting point of tin nanoparticles according to the Lai's model as shown in Fig. 3.1.

\section{Experimental}

To a $50 \mathrm{~mL}$ three-necked round bottom flask, $0.01 \mathrm{~g}$ of $\mathrm{SnI}_{4}(0.016 \mathrm{mmol})$ was dissolved in $10 \mathrm{~mL}$ of TOA $(\geq 98 \%)$ and heated to $150{ }^{\circ} \mathrm{C}$ forming a clear pale yellow solution. An excess of $\mathrm{NaBH}_{4}(0.04 \mathrm{~mL}, 2.0 \mathrm{M}$ in triethylene glycol dimethyl ether) solution was swiftly injected into the hot solution $\left(150{ }^{\circ} \mathrm{C}\right.$ for $1 \mathrm{~min}$ ) followed by quenching the reaction with $30 \mathrm{~mL}$ of toluene. The excess of $\mathrm{NaBH}_{4}$ ensures complete chemical reduction. The resulting pale grey 
precipitate was collected and washed with toluene and ethanol about 5 times, then redispersed in ethanol for subsequent morphology and crystal structure measurements. All the syntheses were carried out under nitrogen flow.

\section{Results and discussion: Reduction at $150{ }^{\circ} \mathrm{C}$}

Fig. 3.2a presents a typical TEM image of the tin nanoparticles prepared at $150{ }^{\circ} \mathrm{C}$. About $80 \%$ of the tin nanoparticle were found in the size ranges of 19 to $31 \mathrm{~nm}$ and $12 \%$ of the particles were around $15 \mathrm{~nm}$ as shown in Fig. 3.2b. The average size of the as-prepared tin nanoparticles is around $25.0 \pm 6.5 \mathrm{~nm}$ (Fig. 3.2).

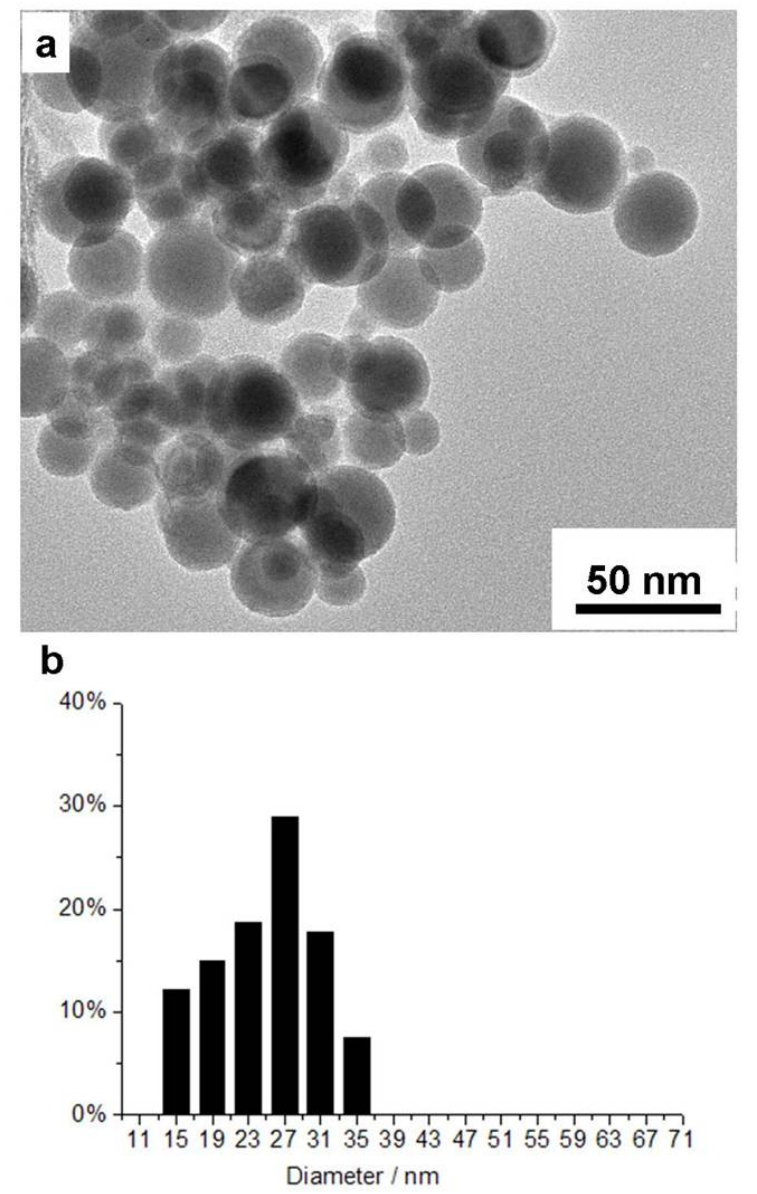

Fig. 3.2 TEM image (a) and histogram (b) of tin nanoparticles synthesis via chemical reduction of $0.016 \mathrm{mmoL} \mathrm{SnI}_{4}$ at $150{ }^{\circ} \mathrm{C}$ in the presence of TOA. 
The HRTEM image as shown in Fig. 3.3 indicates that the nanoparticle has a core/shell structure. The lattice fringes observed in the core confirm the high crystallinity. Although bulk tin has melting point of $231{ }^{\circ} \mathrm{C}$, inspection of tin nanoparticles under electron beam did not reveal any sign of melting. This could be due to the presence of shell. A clearly marked interplanar spacing in the image is $2.92 \AA$, which matches well with (200) atomic planes of the tetragonal structure of $\beta$-Sn (tetragonal, $\mathrm{a}=5.831 \AA, \mathrm{c}=3.182 \AA$ ). ${ }^{19}$ The amorphous shell is about 2 to $3 \mathrm{~nm}$ thick and is expected to be an oxide shell which has also been observed and reported in previous research. ${ }^{12,13}$ The oxide shell may be formed during the purification process in air in which $\sim 100 \mathrm{~mL}$ ethanol was involved.

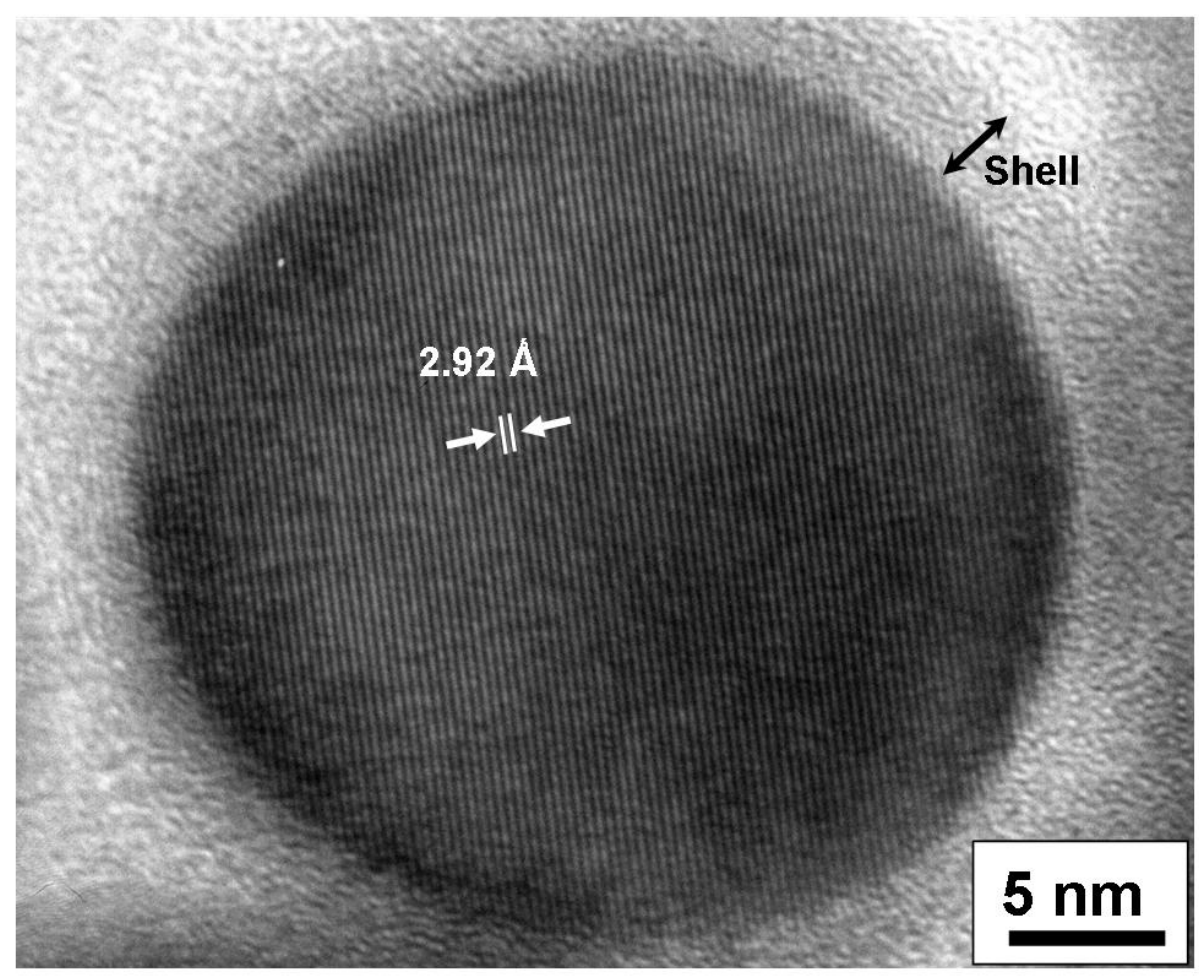

Fig. 3.3 HRTEM image of a single crystal tin nanoparticle.

Fig. 3.4 shows an EDX spectrum from the sample, after purification. EDX analysis confirms that the spherical nanoparticles contain the element tin and oxy- 
gen. It was found that the intensity of element oxygen $(\sim 450$ counts $)$ was almost two times higher than the expected value of 200 counts resulting from the instrument. It further indicated the formation of oxide shells on the tin nanoparticles. The small peaks of sodium are attributed to sodium salt (NaI) which is a by-product of the reaction. It implies that the washing process was not thorough, with trace amounts of by-product still present in the sample. The peaks of carbon and copper on the EDX spectrum is caused by the sample holder (carbon coated copper grid).

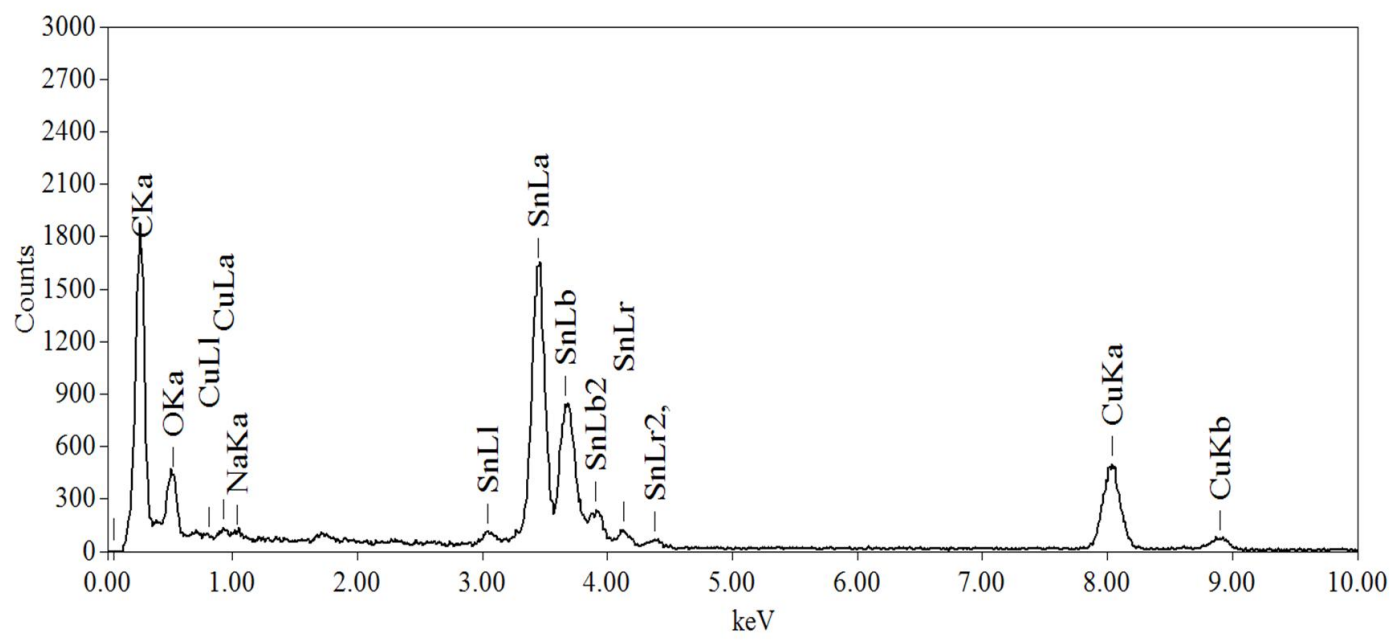

Fig. 3.4 EDX analysis of prepared nanoparticles confirming the presence of Sn.

A typical SAED pattern recorded from the as-prepared tin nanoparticles is shown in Fig. 3.5. The SAED pattern consists of 9 sharp diffraction rings which are marked as numbers in the pattern.

The calibrated camera constant, obtained from the diffraction rings of standard element gold, was used to calculate the d-spacing corresponding to the different radii observed in the diffraction pattern. The results summarized in Table 3.1 are in good agreement with those of $\mathrm{d}$-spacing of tetragonal $\beta$ $\mathrm{Sn}^{19}$ which is the most common crystalline phase of tin obtained in the liter- 
ature. ${ }^{14,20}$ In addition, there is no diffraction ring corresponding to tin oxide phase which is probably indicates that the oxide shell is amorphous.

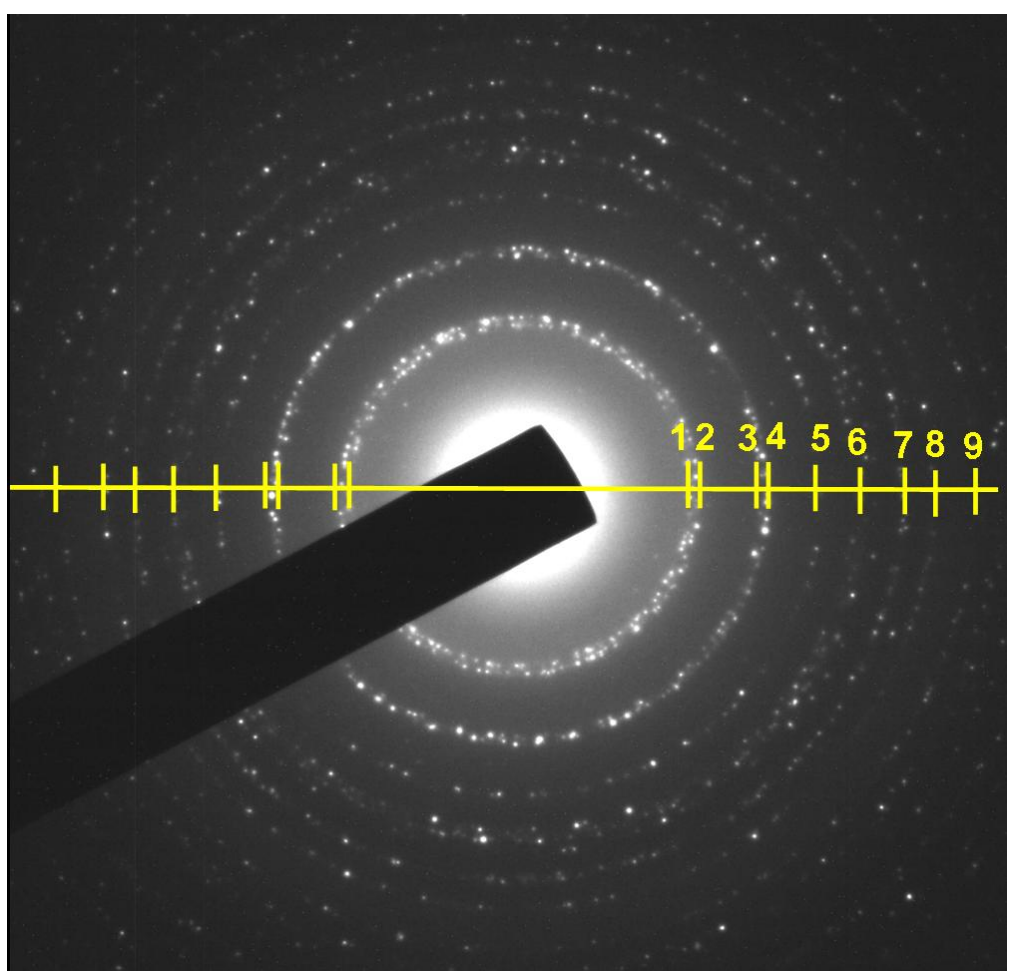

Fig. 3.5 Selected area electron diffraction (SAED) pattern recorded from the as-prepared tin nanoparticles.

Table 3.1 Comparison of $d$-spacing (in $\AA$ ) calculated from SAED with those observed on $\beta$-Sn.

\begin{tabular}{|c|c|c|c|}
\hline No & d-spacing(calculated) & d-spacing (00-004-0673) & hkl \\
\hline 1 & $2.95 \pm 0.02$ & 2.915 & 200 \\
\hline 2 & $2.75 \pm 0.02$ & 2.793 & 101 \\
\hline 3 & $2.06 \pm 0.02$ & 2.062 & 220 \\
\hline 4 & $2.00 \pm 0.02$ & 2.017 & 211 \\
\hline 5 & $1.66 \pm 0.02$ & 1.659 & 301 \\
\hline 6 & $1.48 \pm 0.02$ & 1.484 & $112,400,321$ \\
\hline 7 & $1.29 \pm 0.02$ & 1.292 & 420,411 \\
\hline 8 & $1.20 \pm 0.02$ & 1.205 & 312 \\
\hline 9 & $1.09 \pm 0.02$ & 1.095 & 501 \\
\hline
\end{tabular}

XRD measurements were carried out to further confirm the crystal structure of the as-prepared tin nanoparticles. The XRD pattern, shown in Fig. 3.6, exhibits 11 characteristic peaks of tetragonal crystalline tin which is consistent with the 
result obtained from the SAED pattern in Fig. 3.5. No diffraction peak for tin oxide was observed which further confirm the SAED result.

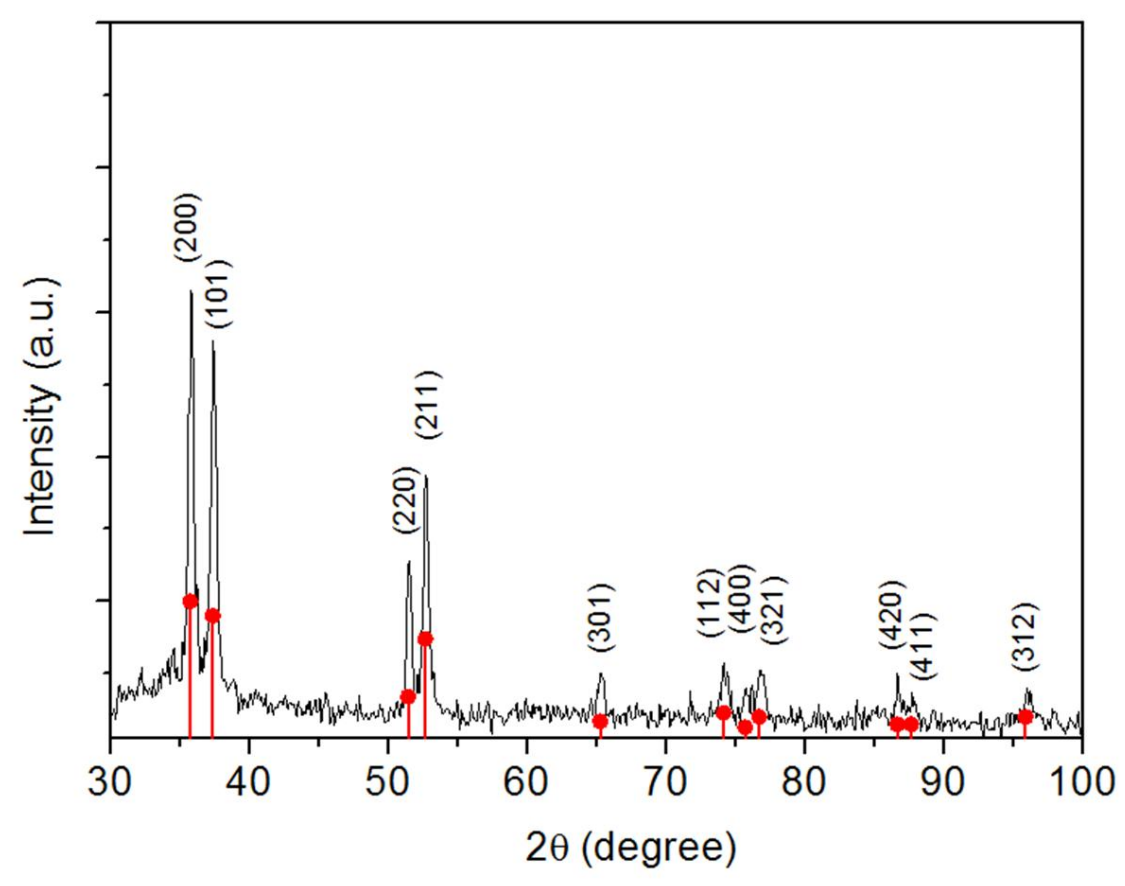

Fig. 3.6 Powder X-ray diffraction for the as-prepared $\mathrm{Sn}$ nanoparticles $(\mathrm{Cu}$ radiation). The expected positions and intensities for peaks corresponding to $\beta$-Sn (ICDD 00-004-0673) are marked by lines labeled with "•".

The core-shell structure and tetragonal crystal structure of tin nanoparticles was also obtained and confirmed in the following experiments.

\subsubsection{Experiment B-C: Effect of reaction temperature}

It is well known that reaction temperature plays a crucial role in determining the nanoparticle size and size distribution. ${ }^{21}$ Increasing the reaction temperature during the reduction of $\mathrm{SnI}_{4}$ was expected to enhance nucleation rate and higher population of tin nanoparticles. Hence, two additional reactions were performed at $200{ }^{\circ} \mathrm{C}$ and $300{ }^{\circ} \mathrm{C}$, keeping other experimental conditions unchanged. The reaction temperature at $200{ }^{\circ} \mathrm{C}$ is close to the melting point of 
tin nanoparticle while the reaction temperature at $300{ }^{\circ} \mathrm{C}$ is above the melting point of tin nanoparticle and even bulk tin. ${ }^{8}$

\section{Results and discussion}

Fig. 3.7a shows a typical TEM image of tin nanoparticles synthesized by chemical reduction of $0.016 \mathrm{mmoL} \mathrm{SnI}_{4}$ at $200{ }^{\circ} \mathrm{C}$.
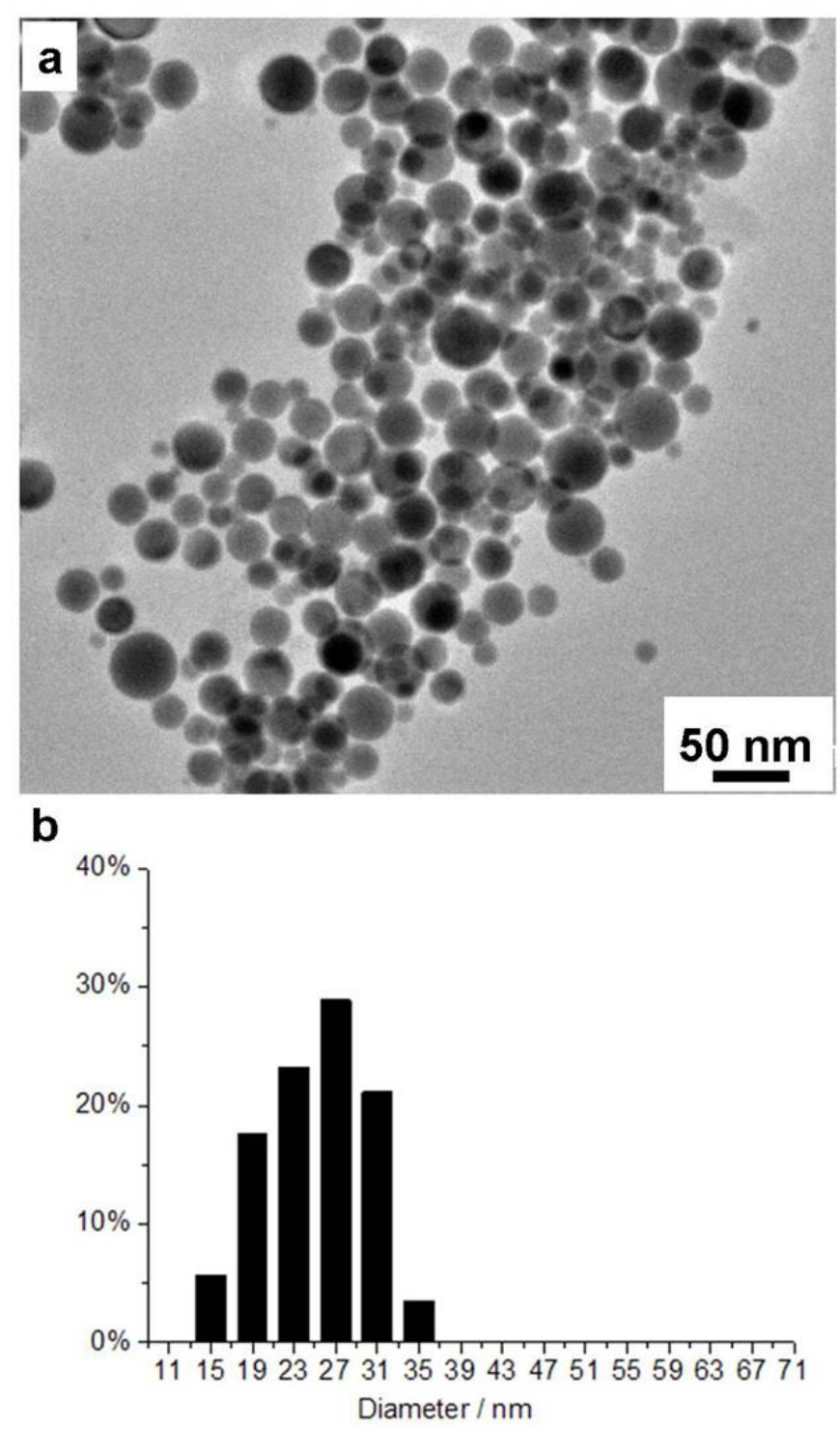

Fig. 3.7 TEM image (a) and histogram (b) of tin nanoparticles synthesis via chemical reduction of $0.016 \mathrm{mmoL} \mathrm{SnI}_{4}$ at $200{ }^{\circ} \mathrm{C}$ in the presence of TOA.

It was observed that the synthesized nanoparticles were spherical in shape 
with smooth surface morphology. These nanoparticles exhibit a relatively narrow size distribution, $91 \%$ of the particles with sizes between 19 and $31 \mathrm{~nm}$. The average size of the nanoparticles estimated from TEM images is around $26.0 \pm 5.5 \mathrm{~nm}$ (Fig. 3.7b).

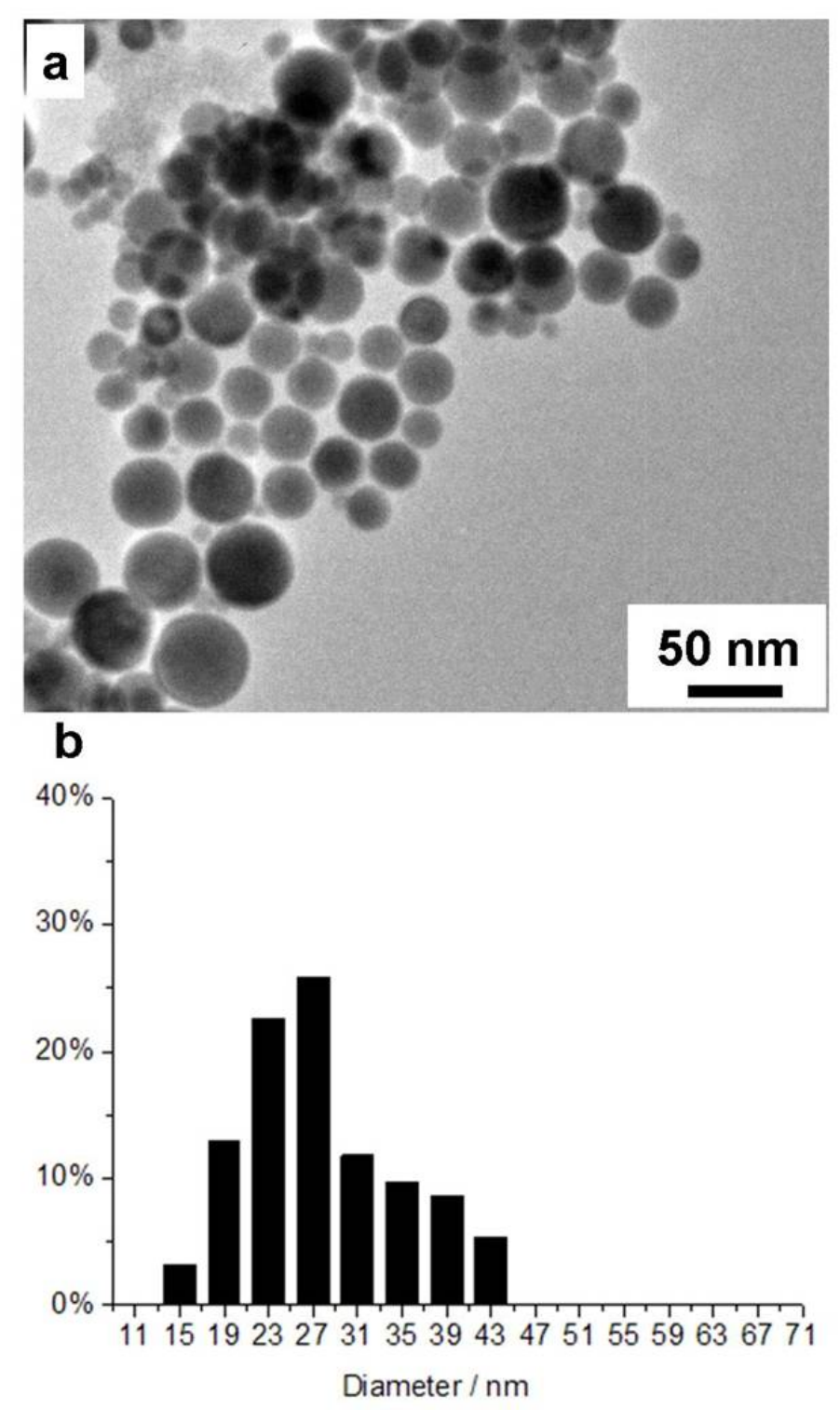

Fig. 3.8 TEM image (a) and histogram (b) of tin nanoparticles synthesis via chemical reduction of $0.016 \mathrm{mmoL} \mathrm{SnI}_{4}$ at $300{ }^{\circ} \mathrm{C}$ in the presence of TOA.

A typical TEM image of the tin nanoparticles prepared at $300{ }^{\circ} \mathrm{C}$ as shown in Fig. 3.8a exhibits a relatively broad size distribution due to the existence of big nanoparticles $(24 \%$ of the particles $>31 \mathrm{~nm}$ ). About $72 \%$ of the nanoparticles 
are in the size range of 19 to $31 \mathrm{~nm}$ (Fig. 3.8b). The average size of the tin nanoparticles is about $28.0 \pm 8.0 \mathrm{~nm}$.

In comparing the size of the tin nanoparticles synthesized at various temperatures: $150{ }^{\circ} \mathrm{C}, 200{ }^{\circ} \mathrm{C}$ and $300^{\circ} \mathrm{C}$ (Table 3.2), it was found that the temperature has very little effect on the particle's average size but it has some effect on size distribution. This may be due to the reaction time (only $1 \mathrm{~min}$ ) not being long enough to result in any significant difference in the temperature-dependent growth.

Table 3.2 Particle size of tin nanoparticles synthesized at different temperatures using $\mathrm{SnI}_{4}$ as tin source.

\begin{tabular}{|c|c|c|c|c|c|}
\hline \multirow{2}{*}{ EXP } & \multirow{2}{*}{ Rxn temp $\left({ }^{\circ} \mathrm{C}\right)$} & \multirow{2}{*}{ Average size $(\mathrm{nm})$} & \multicolumn{3}{|c|}{ Size distribution (\%) } \\
\cline { 4 - 6 } & & & $<19 \mathrm{~nm}$ & $19-31 \mathrm{~nm}$ & $>31 \mathrm{~nm}$ \\
\hline $\mathrm{A}$ & 150 & $25.0 \pm 6.5$ & 12 & 80 & 8 \\
\hline $\mathrm{B}$ & 200 & $26.0 \pm 5.5$ & 6 & 91 & 3 \\
\hline $\mathrm{C}$ & 300 & $28.0 \pm 8.0$ & 3 & 77 & 20 \\
\hline
\end{tabular}

Moveover, Table 3.2 shows that the temperature does affect the nanoparticles's size distributions. At a lower reduction temperature $\left(150^{\circ} \mathrm{C}\right)$, the reaction rate was slow and consequently formed a low concentration of monomers which resulted in both nucleation and slow growth. ${ }^{22}$ As the reaction time is extremely short (only $1 \mathrm{~min}$ ), the formation of tin nanoparticles is dominated by the nucleation process.

It is known that the nucleation dominated process tends to generate small nanoparticles, but these small particles with large surface area to volume ratio possess a higher energy state than larger particles. ${ }^{23}$ Hence, small particles tend to grow or shrink and then form larger particles (Ostwald ripening process $^{24}$ ) which results in a broad size distribution. In contrast at higher reaction temperature $\left(300^{\circ} \mathrm{C}\right)$, the reduction was fast and formed a high concentration 
of monomer which resulted in both the nucleation and growth being too fast to control. Together with the temperature being higher than the melting temperature of tin leads to the formation of liquid tin droplet with a large size distribution. These polydisperse liquid tin droplets are transferred to the solid phase when the temperature is decreased below melting point. When the reaction temperature is set at $200{ }^{\circ} \mathrm{C}$, which is close to the melting point of tin nanoparticles ${ }^{8}$ the synthesized particles exhibited a relatively narrow size distribution(Table 3.2) which indicated that the optimal reaction temperature for the synthesis of tin nanoparticles is $200{ }^{\circ} \mathrm{C}$.

\subsubsection{Experiment D: Effect of high concentration}

Increasing the concentration of $\mathrm{SnI}_{4}$ from $0.0016 \mathrm{mmol} / \mathrm{mL}$ (experiment $\mathrm{A}$ ) to $0.016 \mathrm{mmol} / \mathrm{mL}$, keeping all other synthesis conditions the same (including the $\mathrm{Sn}: \mathrm{NaBH}_{4}$ ratio of 1:5.) as in experiment $\mathrm{B}$, was expected to produce larger size tin nanoparticles.

\section{Results and discussion}

Fig. 3.9a shows a typical TEM image of the produced tin nanoparticles. It was found that the spherical tin nanoparticles tend to aggregate together. More significantly, an increase in the tin precursor concentration also altered the size and size distribution of the resultant tin nanoparticles. From Fig. 3.9b, it can be seen that the resultant particles exhibit a large size distribution (size range between 15 and $59 \mathrm{~nm}$ ). Only $35 \%$ of particles were found within the size range of 19 to $31 \mathrm{~nm}$, which is much lower than that obtained in the experiment B (91 \%) as shown in Table 3.3. The majority of the particles (63\%) are bigger than $31 \mathrm{~nm}$. The average size of the particles increased from $26.0 \pm 5.5 \mathrm{~nm}$ in the typical synthesis to $36.5 \pm 15.5 \mathrm{~nm}$. 

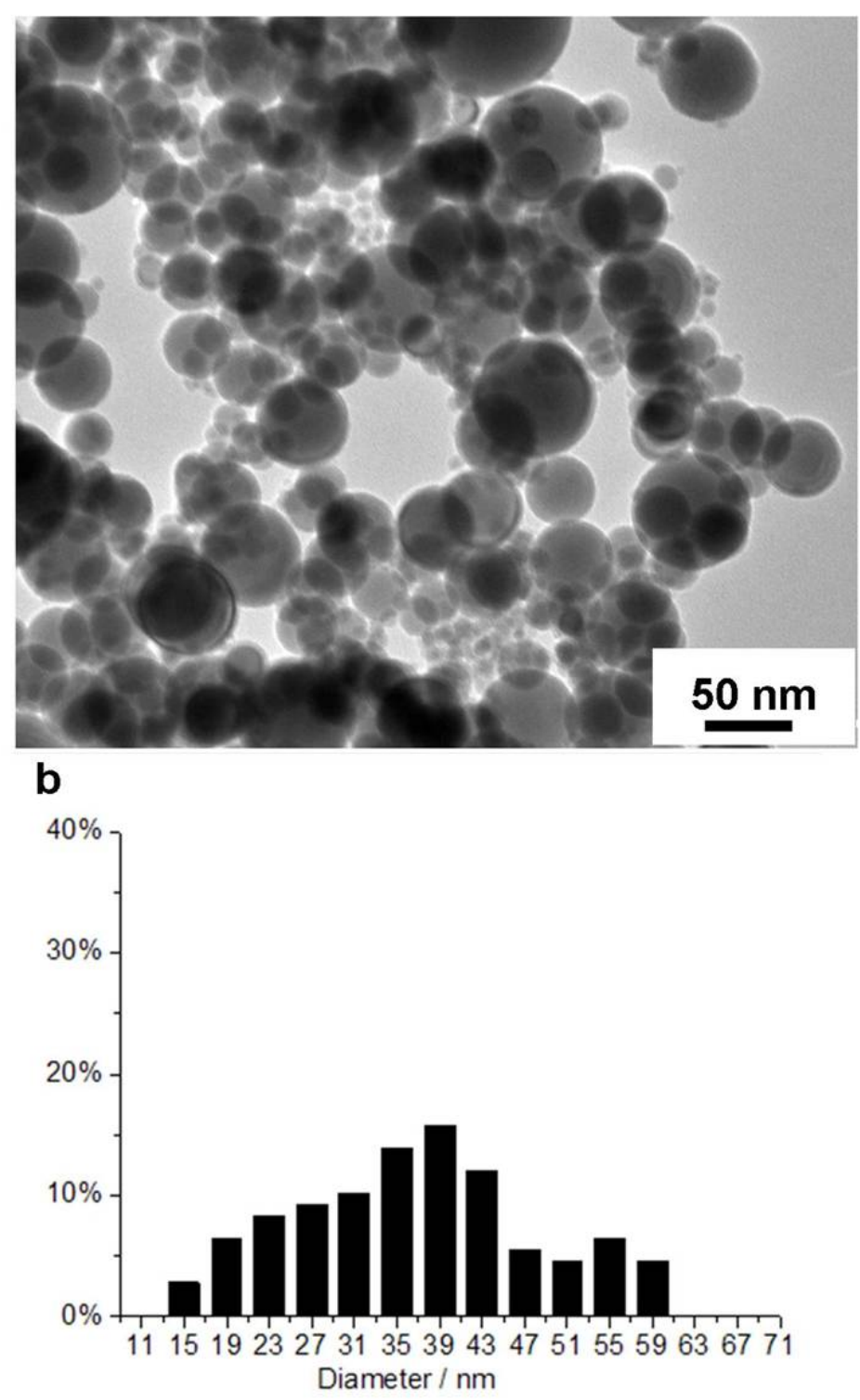

Fig. 3.9 TEM image (a) and histogram (b) of tin nanoparticles synthesis by increasing the precursor concentration.

Table 3.3 Particle size of tin nanoparticles synthesized at different concentration of $\mathrm{SnI}_{4}$.

\begin{tabular}{|c|c|c|c|c|c|}
\hline \multirow{2}{*}{ EXP } & \multirow{2}{*}{ Conc. of tin precursor } & \multirow{2}{*}{ Average size $(\mathrm{nm})$} & \multicolumn{3}{|c|}{ Size distribution (\%) } \\
\cline { 3 - 6 } & & & $<19 \mathrm{~nm}$ & $19-31 \mathrm{~nm}$ & $>31 \mathrm{~nm}$ \\
\hline B & $0.0016 \mathrm{mmol} / \mathrm{mL}$ & $26.0 \pm 5.5$ & 6 & 91 & 3 \\
\hline $\mathrm{D}$ & $0.016 \mathrm{mmol} / \mathrm{mL}$ & $36.5 \pm 15.5$ & 2 & 35 & 63 \\
\hline
\end{tabular}

The observed concentration effect can be explained by three reasons: (1) the concentration of the monomer increases significantly with higher $\mathrm{SnI}_{4}$ concen- 
tration, which results in the formation of larger amount of small nanoparticles. (2) The small particles with higher energy state have the tendency to grow into larger particles. (3) the collision frequency of tin nanoparticles increases significantly with the increasing $\mathrm{SnI}_{4}$ concentration and consequently nanoparticles aggregation and growth take place.

\subsubsection{Experiment E: Effect of surfactant}

The presence of surfactant is essential for the stabilization of colloidal nanoparticles and rendering them dispersible in the solvent. ${ }^{25}$ The average size and size distribution of nanoparticles can be controlled by varying the surfactant. ${ }^{22}$ To investigate the surfactant effect, an additional surfactant, $2 \mathrm{~mL}$ oleylamine (OLA), was added into TOA, keeping the other reaction conditions same as in the experiment $\mathrm{B}\left(200{ }^{\circ} \mathrm{C}\right)$, except for the concentration of $\mathrm{SnI}_{4}$ which slightly decreased from $0.016 \mathrm{mmol}$ to $0.013 \mathrm{mmol}$. This change is considered negligible. OLA is a primary, long chain alkylamine which is considered a relatively strong coordinating ligand and commonly used for the metal nanoparticles synthesis. ${ }^{26}$ Therefore using a mixed surfactant of OLA with TOA in the reaction system was expected to decrease the average size and size distribution of tin nanoparticles.

\section{Results and discussion}

The TEM image of the resultant tin nanoparticles prepared in the presence of OLA is shown in Fig. 3.10a and reveals that the spherical particles are nearly uniform in size. The tin nanoparticles size ranges between 9 and $15 \mathrm{~nm}$. The average size of the particles is about $11.5 \pm 2.0 \mathrm{~nm}$ which is significantly smaller than the average size of the nanoparticle obtained from the typical synthesis. The results described above indicated that the surfactant plays a crucial role in controlling the size and size distribution in the present rapid hot injection 
synthesis method.
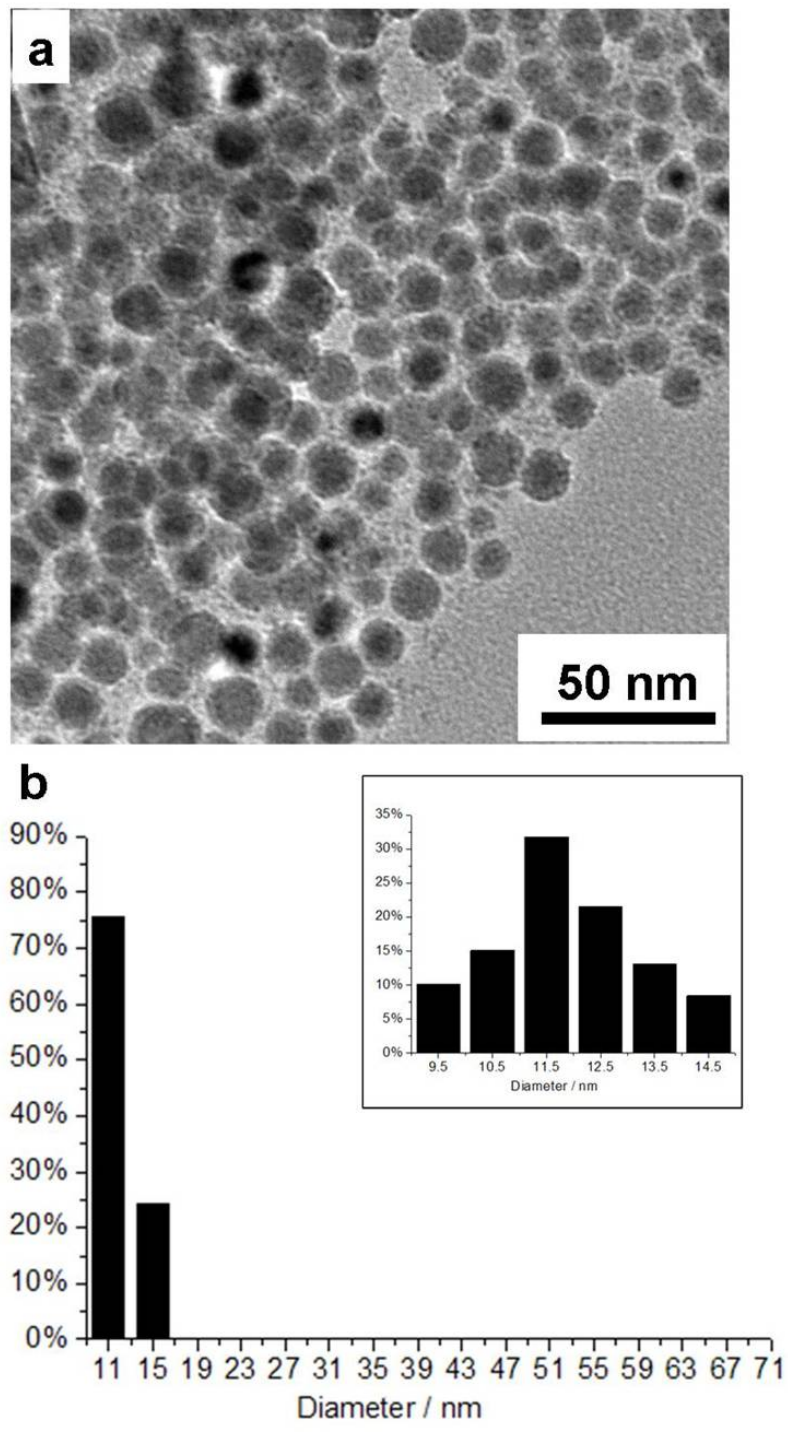

Fig. 3.10 TEM image (a) and histogram (b) of tin nanoparticles synthesis via chemical reduction of $0.016 \mathrm{mmoL} \mathrm{SnI}_{4}$ at $200{ }^{\circ} \mathrm{C}$ in the presence of a mixture of OLA and TOA.

\subsubsection{Experiment F-G: Effect of Tin source}

$\mathrm{SnCl}_{2}$ was used instead of $\mathrm{SnI}_{4}$ to investigate the affect of tin precursor on the synthesis. Two additional experiments were performed at the injection temperatures of $200{ }^{\circ} \mathrm{C}$ and $300{ }^{\circ} \mathrm{C}$ using $\mathrm{SnCl}_{2}$ as tin source. 
The results described in the previous sections are for the tin nanoparticles synthesized using $\mathrm{SnI}_{4}$ as a tin precursor. Previous research has demonstrated that using $\mathrm{Sn}(\mathrm{IV})$ precursor can achieve better size control of tin nanoparticles than using Sn(II) precursor via low temperature $\left(65^{\circ} \mathrm{C}\right)$ chemical reduction. ${ }^{14,20}$

To further demonstrate the important effect of tin precursor, one additional experiment (Experiment F) was performed under conditions identical to the Experiment B, except for the replacement $\mathrm{SnI}_{4}$ with $\mathrm{SnCl}_{2}$ of same mole quantity and adjusting the amount of $\mathrm{NaBH}_{4}$ from $0.08 \mathrm{mmol}$ to $0.04 \mathrm{mmol}$. Apart from the reaction at $200{ }^{\circ} \mathrm{C}$, another reaction (Experiment $\mathrm{G}$ ) was carried out at $300{ }^{\circ} \mathrm{C}$ using $\mathrm{SnCl}_{2}$ as tin precursor, keeping the other reaction conditions unchanged.

\section{Results and discussion}

Fig. 3.11a shows a typical TEM image of tin nanoparticles prepared using $\mathrm{Sn}(\mathrm{II}) \mathrm{Cl}_{2}$ as tin source at $200{ }^{\circ} \mathrm{C}$. The resultant spherical tin nanoparticles are polydisperse and vary in size from 15 to $70 \mathrm{~nm}$. The average size of the nanoparticles is $40.0 \pm 18.5 \mathrm{~nm}$ (Fig. 3.11b). 

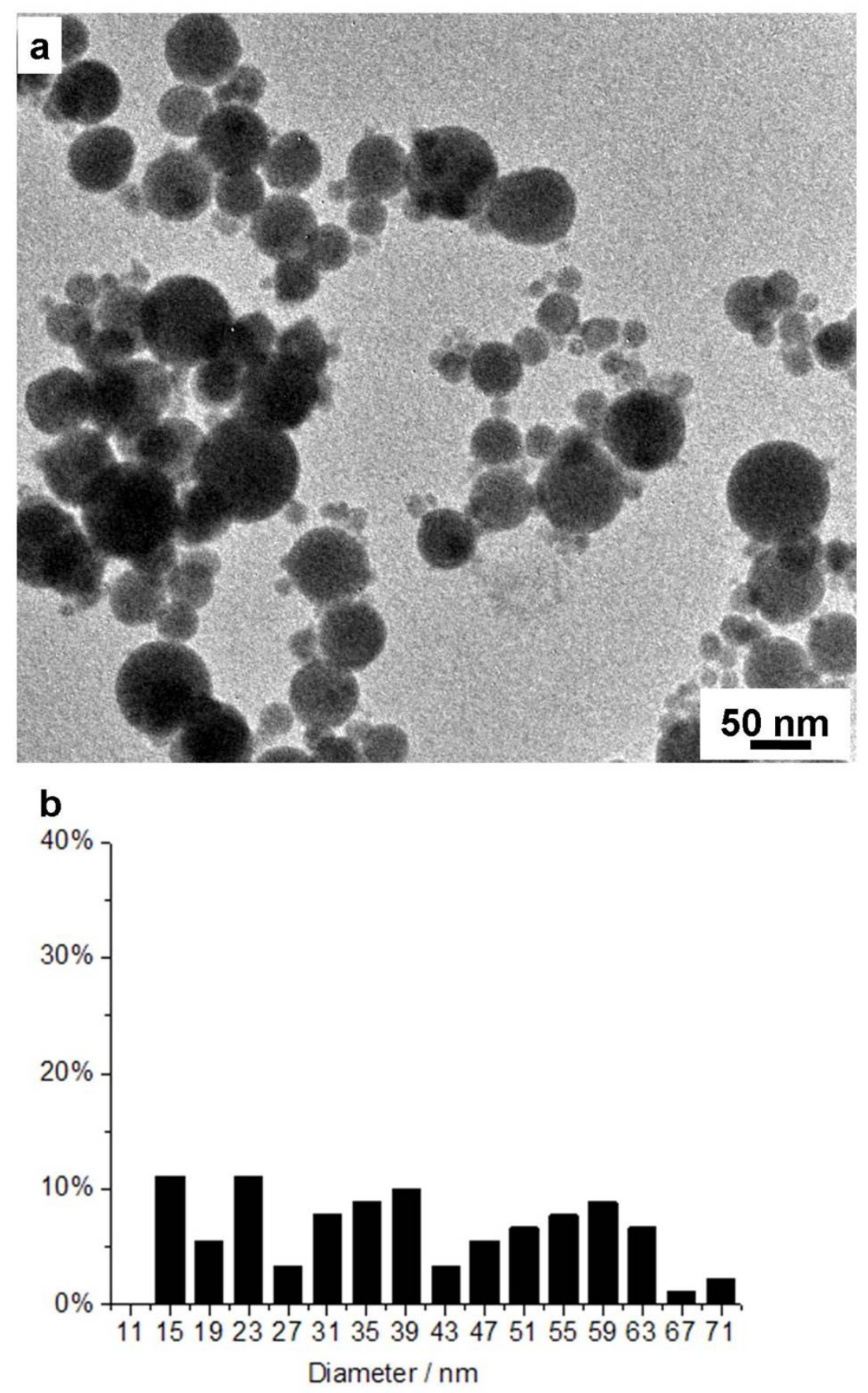

Fig. 3.11 TEM image (a) and histogram (b) of tin nanoparticles synthesis via chemical reduction of $0.016 \mathrm{mmoL} \mathrm{SnCl}_{2}$ at $200{ }^{\circ} \mathrm{C}$ in the presence of TOA.

Fig. 3.12a shows that the TEM image of tin nanoparticles synthesized by using $\mathrm{SnCl}_{2}$ as tin source at $300{ }^{\circ} \mathrm{C}$. It is observed that the particle size is spread over a broad range from 15 to $120 \mathrm{~nm}$. 5\% of these particles were larger than 100 $\mathrm{nm}$ (Fig. 3.12b). The average size of the particles is about $45 \pm 20.0 \mathrm{~nm}$. 


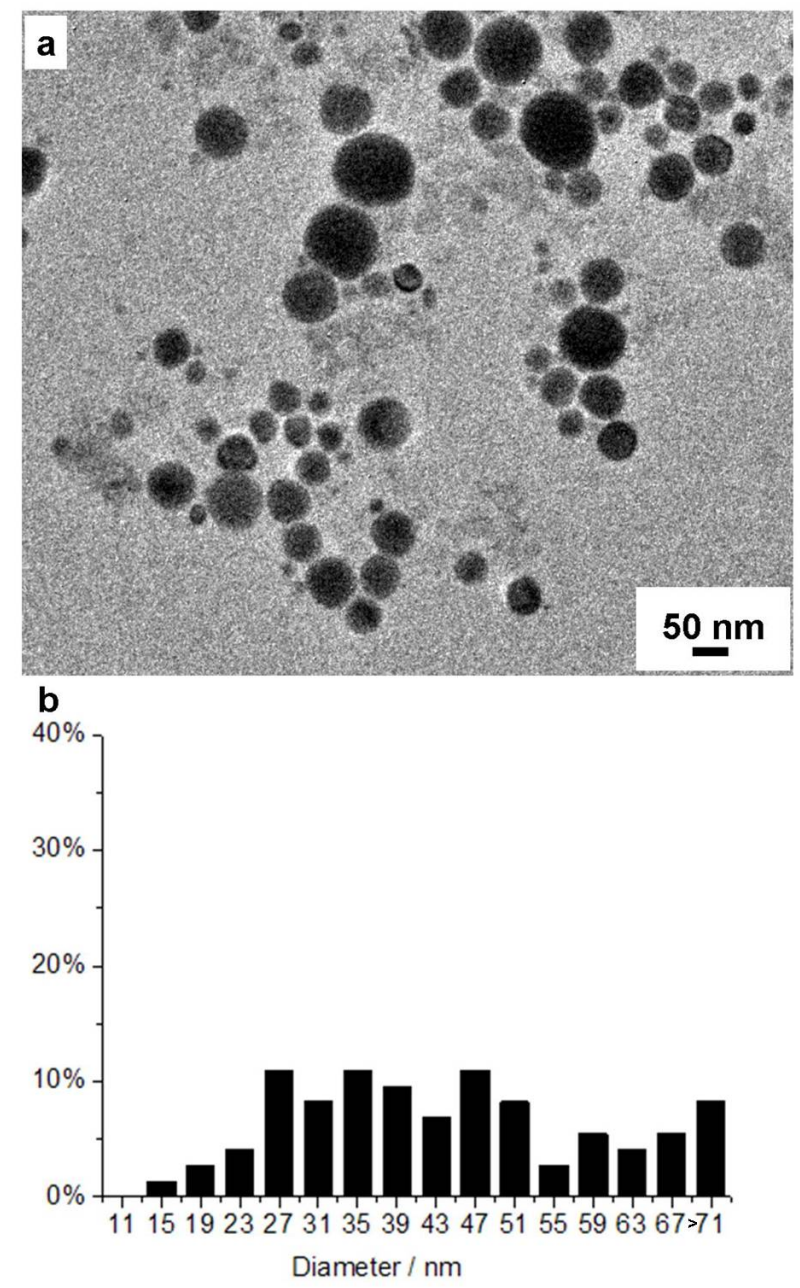

Fig. 3.12 TEM image (a) and histogram (b) of tin nanoparticles synthesis via chemical reduction of $0.016 \mathrm{mmoL} \mathrm{SnCl}_{2}$ at $300{ }^{\circ} \mathrm{C}$ in the presence of TOA.

The experimental results show that highly polydisperse tin nanoparticles were obtained by using $\mathrm{Sn}(\mathrm{II}) \mathrm{Cl}_{2}$ as a tin precursor. The particles in Fig. 3.11 and Fig. 3.12 exhibit multi-modal(polydisperse) distributions which could be due to the uncontrolled growth of the particles due to faster nucleation and growth at high temperature or aggregation due to melting at high temperature. In contrast, using $\mathrm{Sn}(\mathrm{IV}) \mathrm{I}_{4}$ as a tin precursor provides good particle size control. Moreover, comparing Fig. 3.11b and Fig. 3.12b, shows that a higher reaction temperature leads the formation of large particles with a wide size distribution. This observation agrees well with the result observed by using $\mathrm{SnI}_{4}$ as a precursor. 


\subsection{Summary}

In summary, narrow size distribution tin nanoparticles have been successfully prepared by using the "rapid hot-injection" method. Tin nanoparticle was synthesized through chemical reduction tin halide with sodium borohydride. The starting materials are inexpensive and easy to handle and the synthesis procedures are simple. The as-synthesized tin nanoparticles have tetragonal crystal structure. A thin amorphous shell around highly crystalline core was observed.

The tin nanoparticles with an average size ranging from $11 \mathrm{~nm}$ to $40 \mathrm{~nm}$ were synthesized by varying the reaction parameters such as reaction temperature, precursor and surfactant. It was found that an increase or decrease in the reaction temperature did not affect the average particle size but may influence the nanoparticles size distribution during the limited (1 min) reaction time. High temperature synthesis with fast nucleation and growth tends to form bigger particles than those made at low temperature. In addition, an increase in tin precursor concentration results in an enlarged particle size distribution. Adding an additional strong surfactant (OLA) in the reaction system decreased the particle size and produced a narrow particle size distribution. Replacing the $\mathrm{SnI}_{4}$ with $\mathrm{SnCl}_{2}$ in the system increased the particle size and size distribution.

The reaction at $200{ }^{\circ} \mathrm{C}$ using $\mathrm{SnI}_{4}$ in the presence of TOA was found to be the optimal condition with respect to monodispersity of low cost. 


\section{REFERENCES}

[1] Humpston, G.; Jacobson, D. M. Principles of soldering; ASM International, 2004.

[2] Buffat, P.; Borel, J.-P. Phys. Rev. A 1976, 13, 2287-2298.

[3] Bachels, T.; Güntherodt, H.-J.; Schäfer, R. Phys. Rev. Lett. 2000, 85, 12501253.

[4] Wang, L.; Zhang, Y.; Bian, X.; Chen, Y. Physics Letters A 2003, 310, 197-202.

[5] Delogu, F. Journall of Material Science 2008, 43, 26112617.

[6] Lai, S. L.; Guo, J. Y.; Petrova, V.; Ramanath, G.; Allen, L. H. Phys. Rev. Lett. $1996,77,99$.

[7] Bottani, C. E.; Bassi, A. L.; Tanner, B. K.; Stella, A.; Tognini, P.; Cheyssac, P.; Kofman, R. Materials Science and Engineering: C 2001, 15, 41 - 43.

[8] Jiang, H.; sik Moon, K.; Dong, H.; Hua, F.; Wong, C. Chemical Physics Letters 2006, 429, $492-496$.

[9] Trentler, T. J.; Hickman, K. M.; Goel, S. C.; Viano, A. M.; Gibbons, P. C.; Buhro, W. E. Science 1995, 270, 1791-1794.

[10] Lu, X.; Fanfair, D. D.; Johnston, K. P.; Korgel, B. A. Journal of the American Chemical Society 2005, 127, 15718-15719.

[11] Li, Z.; Tao, X.; Cheng, Y.; Wu, Z.; Zhang, Z.; Dang, H. Ultrasonics Sonochemistry 2007, 14, 89-92.

[12] Zhao, Y.; Zhang, Z.; Dang, H. Materials Science and Engineering A 2003, 359, $405-407$. 
[13] Nayral, C.; Viala, E.; Fau, P.; Senocq, F.; Jumas, J.-C.; Maisonnat, A.; Chaudret, B. Chemistry A European Journal 2000, 6, 4082-4090.

[14] Balan, L.; Schneider, R.; Billaud, D.; Ghanbaja, J. Materials Letters 2005, 59, $1080-1084$.

[15] LaMer, V. K.; Dinegar, R. H. Journal of the American Chemical Society 1950, $72,4847-4854$.

[16] Celso, d. M. D.; Peter, L.; Daniel, V. Small 2005, 1, 1152-1162.

[17] Semiconductor nanocrystal quantum dots: synthesis, assembly, spectroscopy; Rogach, A. L., Ed.; Springer Wien New York, 2008; p 44.

[18] SI chemical data, 3rd ed.; Aylward, G., Findlay, T., Eds.; Jacaranda Wiley, 1974.

[19] Swanson, T. author; Technical Report, 1953; p 24.

[20] Balan, L.; Schneider, R.; Billaud, D.; Ghanbaja, J. Nanotechnology 2005, 16, 1153.

[21] Rai, A.; Singh, A.; Ahmad, A.; Sastry, M. Langmuir 2006, 22, 736-741.

[22] Yin, Y.; Alivisatos, A. P. Nature 2005, 437, 664-670.

[23] Youyi, S.; Ding, W.; Jiangang, G.; Zhi, Z.; Qijin, Z. Journal of Applied Polymer Science 2007, 103, 3701-3705.

[24] Park, J. P.; Joo, J.; Kwon, S. G.; Jang, Y. J.; Hyeon, T. Angewandte Chemie International Edition 2007, 46, 4630-4660.

[25] Cozzoli, P. D.; Pellegrino, T.; Manna, L. Chemical Society Reviews 2006, 35, 1195-1208.

[26] Shi, C.; Tian, L.; Wu, L.; Zhu, J. The Journal of Physical Chemistry C 2007, $111,1243-1247$. 


\section{Chapter 4}

\section{Solution Synthesis of Tin}

\section{Germanium Alloy Nanowires}

\section{Contents}

4.1 Introduction . . . . . . . . . . . . . . . . 89

4.2 Synthesis of tin germanium nanowires . . . . . . . 92

4.3 Composition Analysis of tin germanium nanowires $\ldots \ldots 110$

4.4 Local Structure Characterization of Nanowires . . . . . . 114

4.5 Growth Mechanism of Nanowires . . . . . . . . . . . . 121

4.6 Summary ....................... 122

\subsection{Introduction}

This chapter is concerned with the development of a convenient one pot solutionphase synthesis of tin germanium nanowires. The synthetic approach described here involves the reduction of a tin halide and triphenylgermanium chloride $\left(\mathrm{Ge}(\mathrm{Ph})_{3} \mathrm{Cl}\right)$ with sodium borohydride $\left(\mathrm{NaBH}_{4}\right)$ in the presence of trioctylamine via Solution-Liquid-Solid (SLS) growth. 
Research on the synthesis of semiconductor nanowires has increased remarkably over the past decades. ${ }^{1-4}$ These one dimensional nanostructures offer unique properties such as quantum confinement size effect, enhanced surface sensitivity, high aspect ratio (length to diameter ratio) and large surface to volume ratio compared to their bulk materials and consequently they have great potential applications in electronics, ${ }^{5}$ photonics, ${ }^{6}$ energy, ${ }^{7,8}$ field emitters, ${ }^{9}$ sensors ${ }^{10}$ and biotechnology. ${ }^{11}$

Tin germanium alloys have attracted much attention as they possess a direct and tunable band gap for certain stoichiometries. From theoretical prediction, the direct band gap of $\mathrm{Sn}_{x} \mathrm{Ge}_{1-x}$ alloy of approximately $0.55 \mathrm{eV}<\mathrm{E}_{g}<0 \mathrm{eV}$ for $0.2<\mathrm{x}<0.6 .^{12,13}$ Recent experimental result has demonstrated that the band gap of $\mathrm{Sn}_{x} \mathrm{Ge}_{1-x}$ will change from indirect band gap into direct band gap when the mole fraction of $\mathrm{Sn}$ is larger than the critical ratio of around $0.10 .{ }^{14}$ Moreover, the optical absorption measurements for $\mathrm{Sn}_{x} \mathrm{Ge}_{1-x}$ alloy films indicate strong interband transitions with a direct energy gap of $0.35 \mathrm{eV}<\mathrm{E}_{g}<0.8 \mathrm{eV}$ for composition $0.15>x>0 .{ }^{15}$ These unusual and interesting electronic properties together with their inexpensive materials costs and low toxicity make them ideal candidates for future potential applications in infrared detector, photovoltaics and biomedical fields. ${ }^{16,17}$ Current research on the synthesis of $\mathrm{Sn}_{x} \mathrm{Ge}_{1-x}$ nanowires was motivated by these potentials.

The synthesis of $\mathrm{Sn}_{x} \mathrm{Ge}_{1-x}$ is complicated by the limited solubility of $\mathrm{Sn}$ in Ge, which is less than $1 \%$ in the bulk solid, ${ }^{18}$ and a tendency for Sn surface segregation due to a lower Sn surface free energy than that of Ge. ${ }^{19}$ Moreover, the lattice mismatch between $\alpha$-Sn $(\mathrm{a}=6.489 \AA)$ and $\mathrm{Ge}(\mathrm{a}=5.658 \AA)$ is large $(\sim 14 \%)$ and $\alpha$-Sn transforms to $\beta$-Sn at $13.2{ }^{\circ} \mathrm{C} .{ }^{20}$ Nonetheless, a few synthetic 
approaches of bulk $\mathrm{Sn}_{x} \mathrm{Ge}_{1-x}$ alloy films with $\mathrm{Sn}$ composition in the range of 0 $<\mathrm{x}<0.18$ have been developed. ${ }^{15,19}$ Taraci et al. ${ }^{19}$ reported that $\mathrm{Sn}_{x} \mathrm{Ge}_{1-x}$ film with Sn content ranging from 0.09 to 0.15 can be prepared through ultrahighvacuum chemical vapor deposition (CVD) system, where $\left(\mathrm{GeH}_{3}\right)_{2}$ was reacted with $(\mathrm{Ph}) \mathrm{SnD}_{3}$ at $350{ }^{\circ} \mathrm{C}$ on $\mathrm{Si}$ substrates. Guevara and coworkers ${ }^{21}$ prepared $\mathrm{Sn}_{x} \mathrm{Ge}_{1-x}$ by magnetron sputtering system with a base pressure better than $10^{-7}$ mbar. The Sn concentration in $\mathrm{Sn}_{x} \mathrm{Ge}_{1-x}$ was up to $0.14 .{ }^{21}$ He et al. presented a synthesis of $\mathrm{Sn}_{x} \mathrm{Ge}_{1-x}$ with $\mathrm{Sn}$ composition in the range of $0<\mathrm{x}<$ 0.15 by ion-assisted molecular beam epitaxy with a base pressure of about $3 \times$ $10^{-10}$ Torr. ${ }^{15}$ Kouvetakis and coworkers prepared $\mathrm{Sn}_{x} \mathrm{Ge}_{1-x}$ film with $\mathrm{x}$ up to $\sim 0.18$ through a CVD method using $\mathrm{SnD}_{4}$ and $\mathrm{Ge}_{2} \mathrm{H}_{6}$ as precursor at $350{ }^{\circ} \mathrm{C} .{ }^{22}$ Many of the synthetic approaches mentioned above require extreme reaction conditions. To date, the synthesis of one dimensional homogenous $\mathrm{Sn}_{x} \mathrm{Ge}_{1-x}$ nanowires is still a significant challenge.

In the previous decades, many growth technologies, including chemical vapor deposition(CVD), ${ }^{23}$ metalorganic chemical vapor deposition (MOCVD), ${ }^{24}$ vapor-liquid-solid (VLS), ${ }^{25}$ supercritical fluid-liquid-solid (SFLS) ${ }^{26}$ and solutionliquid-solid (SLS) ${ }^{27}$ growth mechanism have been developed to grow semiconductor nanowires. Among them, SLS growth is one of the most common methods used to grow large scale and highly crystalline nanowires. The growth mechanism of SLS is analogous to VLS growth (see detail in section 1.2.2), in which a metal catalyst such as $\mathrm{Bi}, \mathrm{Ni}$ and $\mathrm{Au}$ is used. ${ }^{4,28,29}$ In this work, $\mathrm{Sn}_{1-x} \mathrm{Ge}_{x}$ nanowires were synthesized through a self-catalyzed process in which the alloy nanowires were grown from Sn droplets. The Sn droplets also serve as Sn source during the growth process.

The objectives of this research were: (1) to synthesize a high yield $(>60 \%)$ of 
straight $\mathrm{Sn}_{x} \mathrm{Ge}_{1-x}$ nanowires with a high aspect ratio(>100); (2) to investigate the chemical composition of the nanowires obtained under optimal reaction conditions; (3) to study the local crystal structure of the nanowires, understanding the predominant growth direction, crystal defects and the crystal structure of the interface (the seed/the wire) of the nanowires; (4) to propose the growth mechanism of $\mathrm{Sn}_{x} \mathrm{Ge}_{1-x}$ nanowires.

This chapter includes four main sections: section 4.2 describes synthesis processes of $\mathrm{Sn}_{x} \mathrm{Ge}_{1-x}$ nanowires. The morphologies and the optimum reaction conditions have been worked out; section 4.3 delineates composition analysis of the sample synthesized under optimum reaction conditions, section 4.4 describes local crystal structure characterization of the nanowires and section 4.5 discusses the growth mechanism.

The morphology, structure and composition of as prepared nanowires were studied by TEM, STEM, SEM and EDX. The crystal structure of as-prepared products were obtained by XRD. TEM and STEM samples were prepared by dropping a dilute toluene solution of nanowires onto 'holey' carbon grids. SEM and XRD samples were prepared by drop casting the nanowires from toluene dispersion on a single crystal silicon substrates.

\subsection{Synthesis of tin germanium nanowires}

The synthesis of tin germanium nanowires were based on the SLS growth mechanism. Sn nanoparticles were first synthesized through the method presented in Chapter 3 by reduction of low cost tin halide with sodium borohydride in trioctylamine (TOA). $\mathrm{Ge}^{0}$ was formed by reduction of a germanium precursor with sodium borohydride followed by dissolution in Sn nanoparti- 
cles to form tin germanium alloy nanowires.

Typically, germanium precursors used in SLS growth of Ge nanowire contain Ge in the +4 oxidation state, such as $\mathrm{Ge}\left(\mathrm{CH}_{3} \mathrm{CH}_{2}\right)_{4}{ }^{30}\left(\mathrm{C}_{6} \mathrm{H}_{5}\right)_{2} \mathrm{H}_{2} \mathrm{Ge}^{31} \mathrm{C}_{6} \mathrm{H}_{5} \mathrm{GeCl}_{3}{ }^{32}$ or $\left.\left[\left(\mathrm{CH}_{3}\right)\left(\mathrm{CH}_{2}\right)_{7} \mathrm{CH}=\mathrm{CH}\left(\mathrm{CH}_{2}\right)_{7} \mathrm{CH}_{2} \mathrm{NH}_{2}\right)_{4} \mathrm{Ge}\right]^{4+} \cdot\left(\mathrm{Cl}^{-}\right)_{4} \cdot{ }^{3}$ The use of the $\mathrm{Ge}^{4+}$ precursor germanium triphenylchloride $\left(\left(\mathrm{C}_{6} \mathrm{H}_{5}\right)_{3} \mathrm{GeCl}\right.$ has not been investigated. Hence, in the current research, this was used as a Ge precursor for the synthesis of tin germanium alloy nanowires.

Trioctylamine (TOA) was used as a solvent and surfactant in the synthesis. It enables tuning the reaction temperature over a broad range (up to $300{ }^{\circ} \mathrm{C}$ ) and can also stabilize the nanowires. It has been reported in the synthesis of Ge nanowires. $^{3}$

Small amount of oleylamine (OLA) $(2 \mathrm{~mL})$ was used to prepare $\mathrm{Ge}(\mathrm{Ph})_{3} \mathrm{Cl}$ solution as it can dissolve $\mathrm{Ge}(\mathrm{Ph})_{3} \mathrm{Cl}$ more easily than TOA.

Size distribution analysis, including both diameter and length, was performed by measuring the size of around 100 nanowires for each sample. The diameter of the nanowire was measured from TEM images and the length of the nanowire was obtained from either TEM images (length $<500 \mathrm{~nm}$ ) or SEM images (length $\geq 500 \mathrm{~nm}$ ).

\subsubsection{Experiment A: Reaction at $200{ }^{\circ} \mathrm{C}$}

Initially, tin germanium alloy nanowires were synthesized through the reduction of $\mathrm{SnCl}_{2}$ and $\mathrm{Ge}(\mathrm{Ph})_{3} \mathrm{Cl}$ with $\mathrm{NaBH}_{4}$, in turn, at $200{ }^{\circ} \mathrm{C}$ in the presence of TOA. The molar ratio of Sn/Ge precursors was set as 1: 10. The reaction was performed under a nitrogen atmosphere. 


\section{Experimental}

In a three necked round bottom flask, $0.0031 \mathrm{~g}(0.016 \mathrm{mmol}) \mathrm{SnCl}_{2}$ (anhydrous, $97 \%$, Sigma Aldrich) was dissolved in $10 \mathrm{~mL}$ TOA ( $\geq 98 \%$, Acros organics) at $200{ }^{\circ} \mathrm{C}$ forming a clear colourless solution. In a separate $5 \mathrm{~mL}$ round bottom flask, $0.0543 \mathrm{~g}(0.16 \mathrm{mmol}) \mathrm{Ge}(\mathrm{Ph})_{3} \mathrm{Cl}(\mathrm{ABCR})$ was mixed with $2 \mathrm{~mL}$ OLA (Acros organics $)$ at $100{ }^{\circ} \mathrm{C}$ for 1 hour forming a clear yellow solution. $\mathrm{NaBH}_{4}(0.35 \mathrm{~mL}$, 2.0 M in triethylene glycol dimethyl ether, Sigma Aldrich) was swiftly injected into the tin precursor solution immediately forming a grey solution. Then, the $\mathrm{Ge}(\mathrm{Ph})_{3} \mathrm{Cl}$ solution was swiftly introduced $(\sim 0.5 \mathrm{~mL} / \mathrm{s})$ into the flask. The reaction mixture was heated at $200{ }^{\circ} \mathrm{C}$ for four hours, then left to cool to room temperature naturally. The precipitate was collected and washed with toluene for 5 times. The final product was redispersed in $5 \mathrm{~mL}$ toluene.

\section{Results and discussion}

Fig. 4.1a shows a typical TEM image of the sample prepared at $200{ }^{\circ} \mathrm{C}$. It was found that around $95 \%$ of the product was nanoparticles with roughly spherical shape of size around $60 \pm 30 \mathrm{~nm}$ (Fig. 4.1b) while a few of short nanorods (Length: $100 \mathrm{~nm}$ ) were also observed Fig. 4.1a.
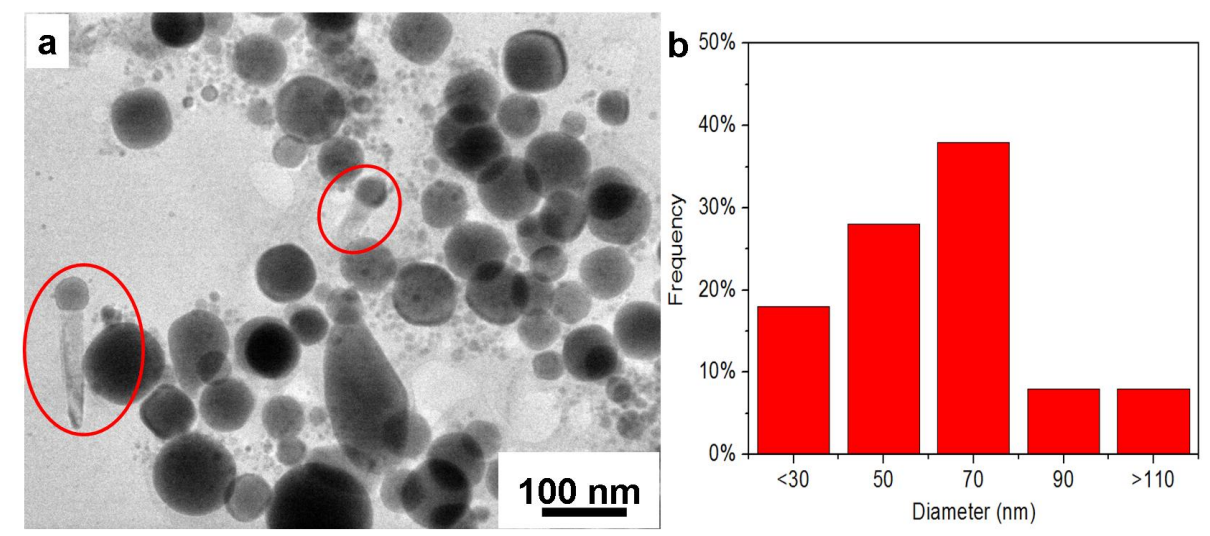

Fig. 4.1 (a)A typical TEM image of the product synthesized at $200{ }^{\circ} \mathrm{C}$. (b) The corresponding diameter distribution profile. 
A typical EDX spectrum (Fig. 4.2)of the product obtained at $200{ }^{\circ} \mathrm{C}$ showed that the atomic ratio of $\mathrm{Sn}$ : Ge was $1: 0.045$ which is significantly lower than that of $\mathrm{Sn} / \mathrm{Ge}$ precursors $(1: 10)$. This is probably due to the slow reduction rates of germanium precursor at a relatively low temperature, $200{ }^{\circ} \mathrm{C}$, and most of precursor has not been reduced.

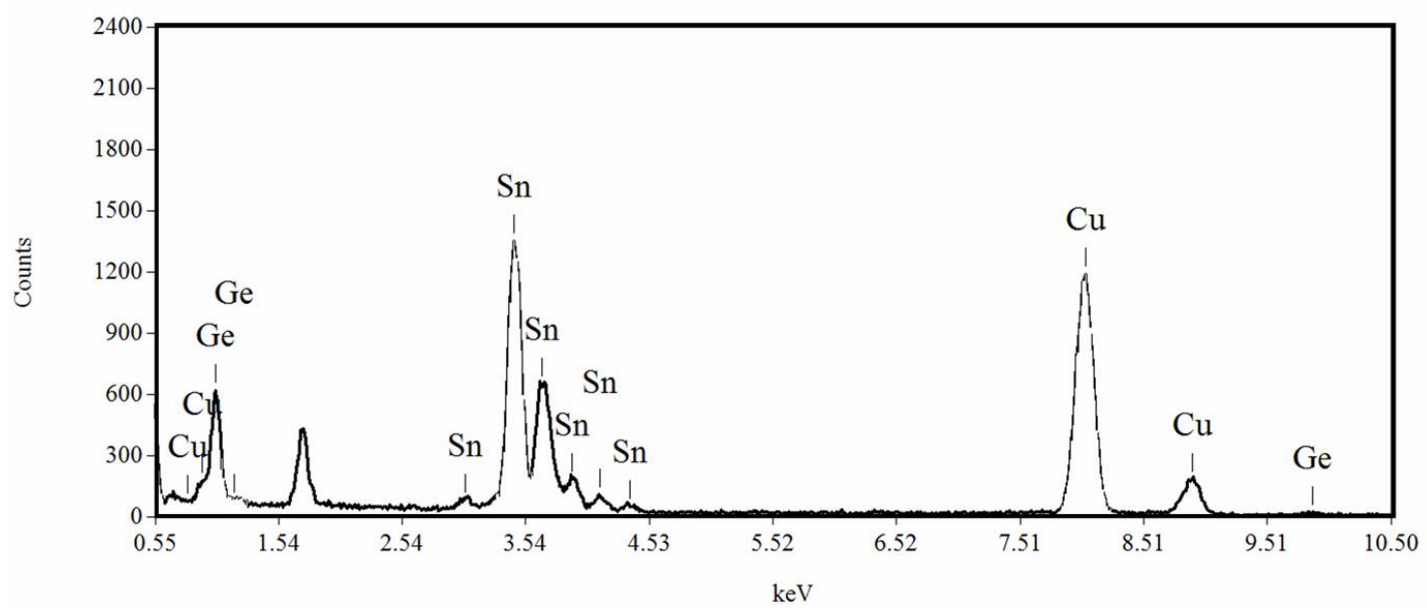

Fig. 4.2 EDX spectrum of the product obtained at $200^{\circ} \mathrm{C}$.

XRD measurement (Fig. 4.3) shows that the appearance of both $\alpha$-Sn (cubic) and $\beta$-Sn (tetragonal) in the product. It is well known that the transformation from pure $\beta$-Sn into $\alpha$-Sn occurs at the temperature below than $13.2^{\circ} \mathrm{C} .{ }^{20}$ But, previous research also found that the presence of aluminum, zinc, copper or germanium element can accelerate this transformation. ${ }^{33}$ Hence, the detected $\alpha$ cubic phase $S n$ in the product can be attributed to the presence of trace amount of germanium. This is the first time the existence of $\alpha$-Sn at room temperature $\left(\sim 20^{\circ} \mathrm{C}\right)$ is reported. No peak observed belongs to Ge which may be due to the low concentration of germanium in the product. This is consistent with EDX measurement result from the same sample. 


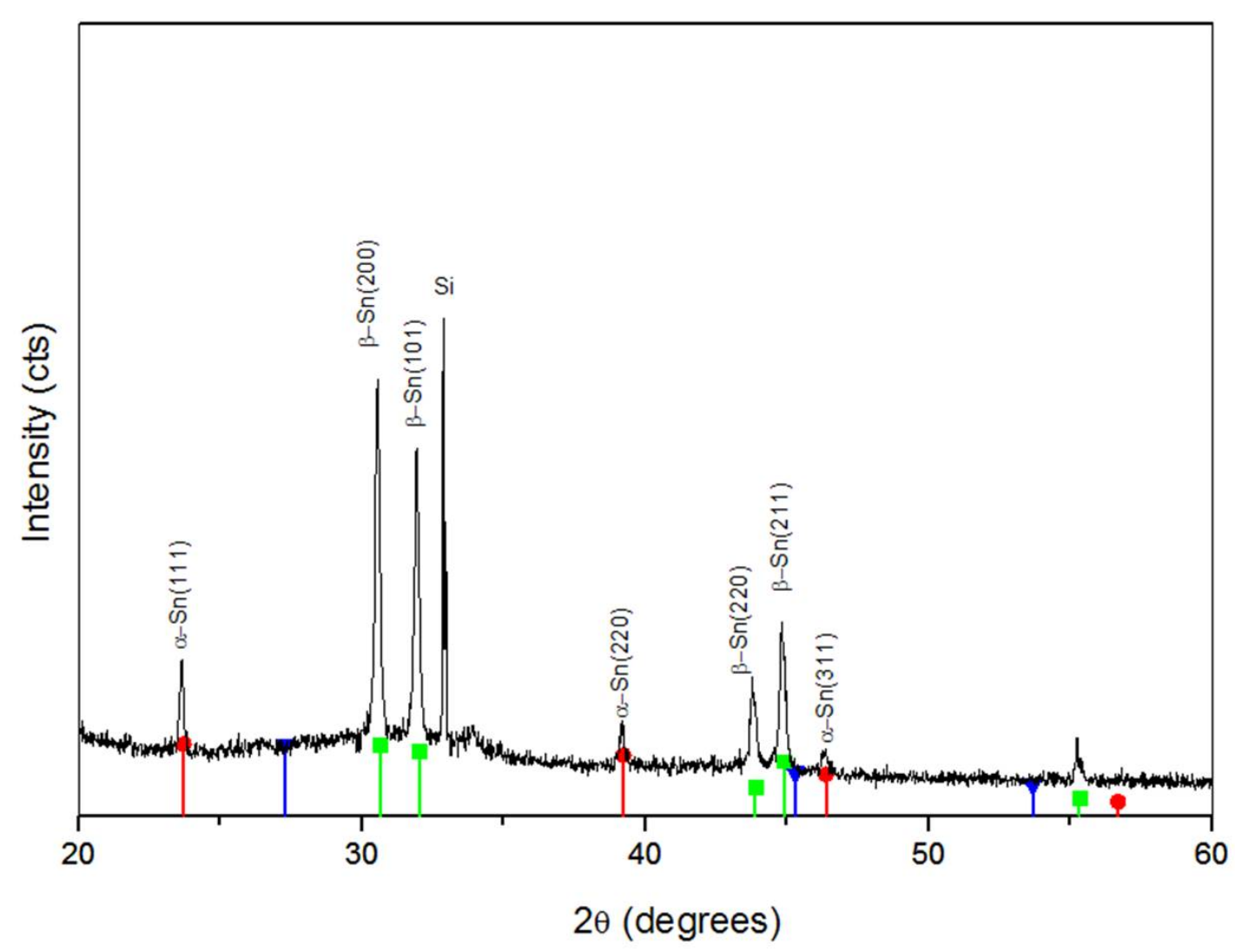

Fig. 4.3 A XRD pattern of as-prepared product ( $\mathrm{Cu}$ radiation). The expected positions and intensities for peaks corresponding to cubic Sn (ICDD 00-005039), cubic Ge (ICDD 00-004-0545) and tetragonal Sn (ICDD 00-004-0673) are

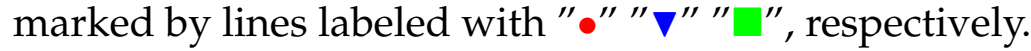

\subsubsection{Experiment B: Reaction at $300{ }^{\circ} \mathrm{C}$}

Increasing the reaction temperature was expected to increase the reduction rate and accelerate the dissolution rate of formed $\mathrm{Ge}^{0}$ in $\mathrm{Sn}$ droplets. In this experiment, the reaction was carried out at $300{ }^{\circ} \mathrm{C}$ and the other reaction conditions were identical to that in experiment $\mathrm{A}$.

\section{Results and discussion}

Fig. 4.4a shows a typical TEM image of the nanowires obtained at $300{ }^{\circ} \mathrm{C}$. The sample composed of early grown $\mathrm{Sn}_{x} \mathrm{Ge}_{1}-x$ nanowires on the $\mathrm{Sn}$ seeds as well as some surfactant and byproducts. The as-prepared nanowires are generally 
straight and short with a length of $130 \pm 40 \mathrm{~nm}$. The diameter of the nanowires is about $50 \pm 20 \mathrm{~nm}$. A HRTEM image, shown in Fig. 4.4b, indicated that the highly crystalline nanowire grew from a Sn nanoparticle. This confirms that the nanowires grew via SLS mechanism.
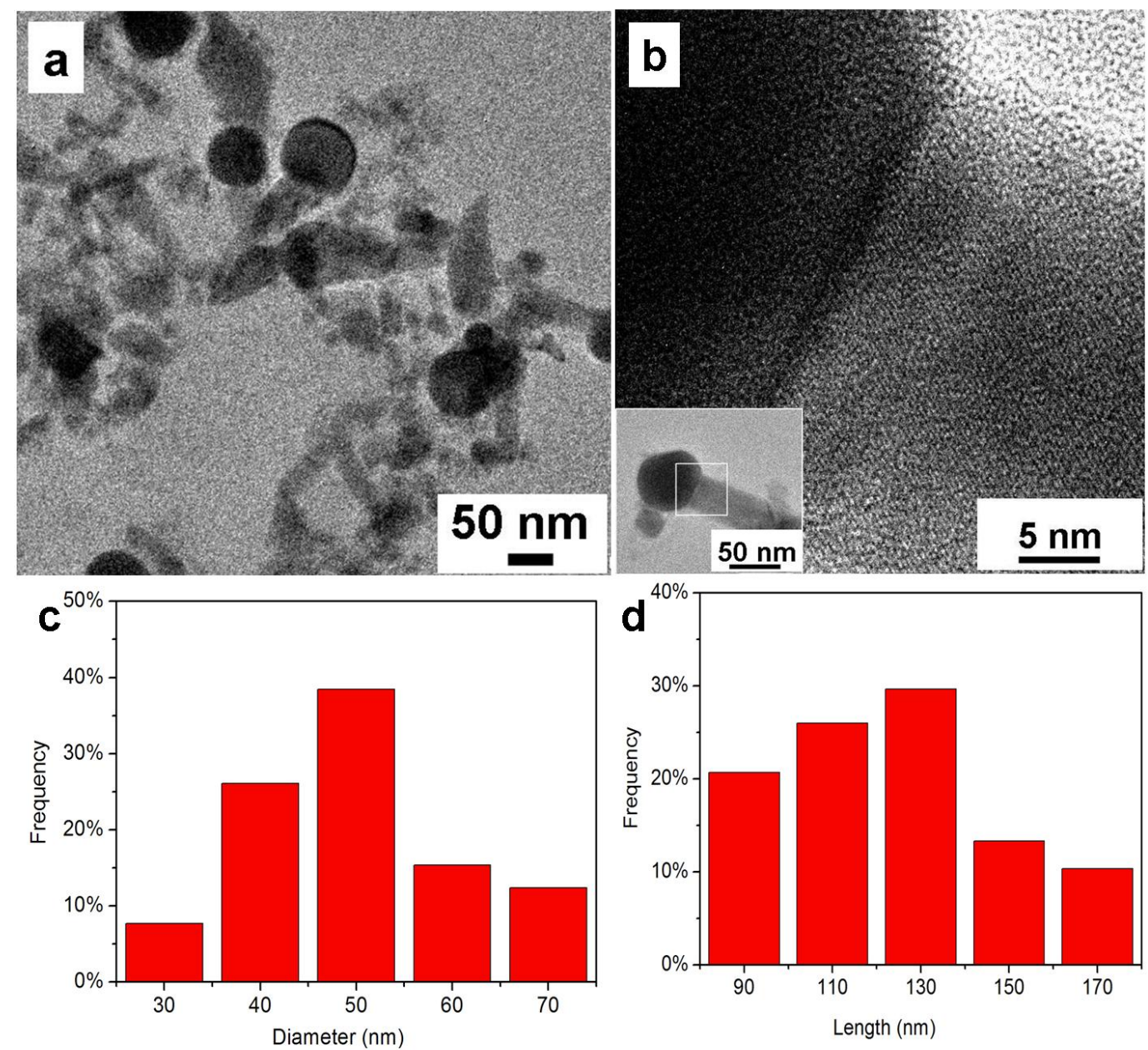

Fig. 4.4 (a) A typical LRTEM image of the product synthesized at $300{ }^{\circ} \mathrm{C}$ via swiftly injection; (b) and (c) The corresponding diameter and length distribution profiles.

Fig. 4.5 presents EDX spectra of a single tin germanium nanowire. The EDX measurement result from the seed region (black region, Fig. 4.5a) shows both Ge and Sn with an atomic ratio of $\sim 1: 18$ which indicates the formation of Sn rich tin germanium alloy. Similar alloy such as $\mathrm{NiGex}^{4}$ or $\mathrm{BiGex}^{29}$ were detected at $\mathrm{Ni}$ or $\mathrm{Bi}$ seed region when they were used to assist Ge nanowire 
growth. The formation of Sn rich tin germanium alloy at seed region is consistent with the SLS growth mechanism in which $\mathrm{Ge}^{0}$ was expected to dissolve in the Sn droplets. In contrast, the EDX measurement result (Fig. 4.5b) from the nanowire core shows the ratio of $\mathrm{Ge} / \mathrm{Sn}$ was $2.2: 1$ which means the formation of Ge rich tin germanium alloy. This observation is different to the previous research, like Bi or Ni seeded Ge nanowire growth in which only Ge was detected from the wire core. ${ }^{4,29}$ This was attributed to the difference among the phase diagrams of $\mathrm{Sn}-\mathrm{Ge}, \mathrm{Bi}-\mathrm{Ge}$ and Ni-Ge systems. In addition, it was also reported that $\mathrm{Sn}$ can be slightly soluble in Ge (the solubility of $\sim 1.14$ atomic $\%$ for $\mathrm{Sn}$ in $\mathrm{Ge}) .^{18}$
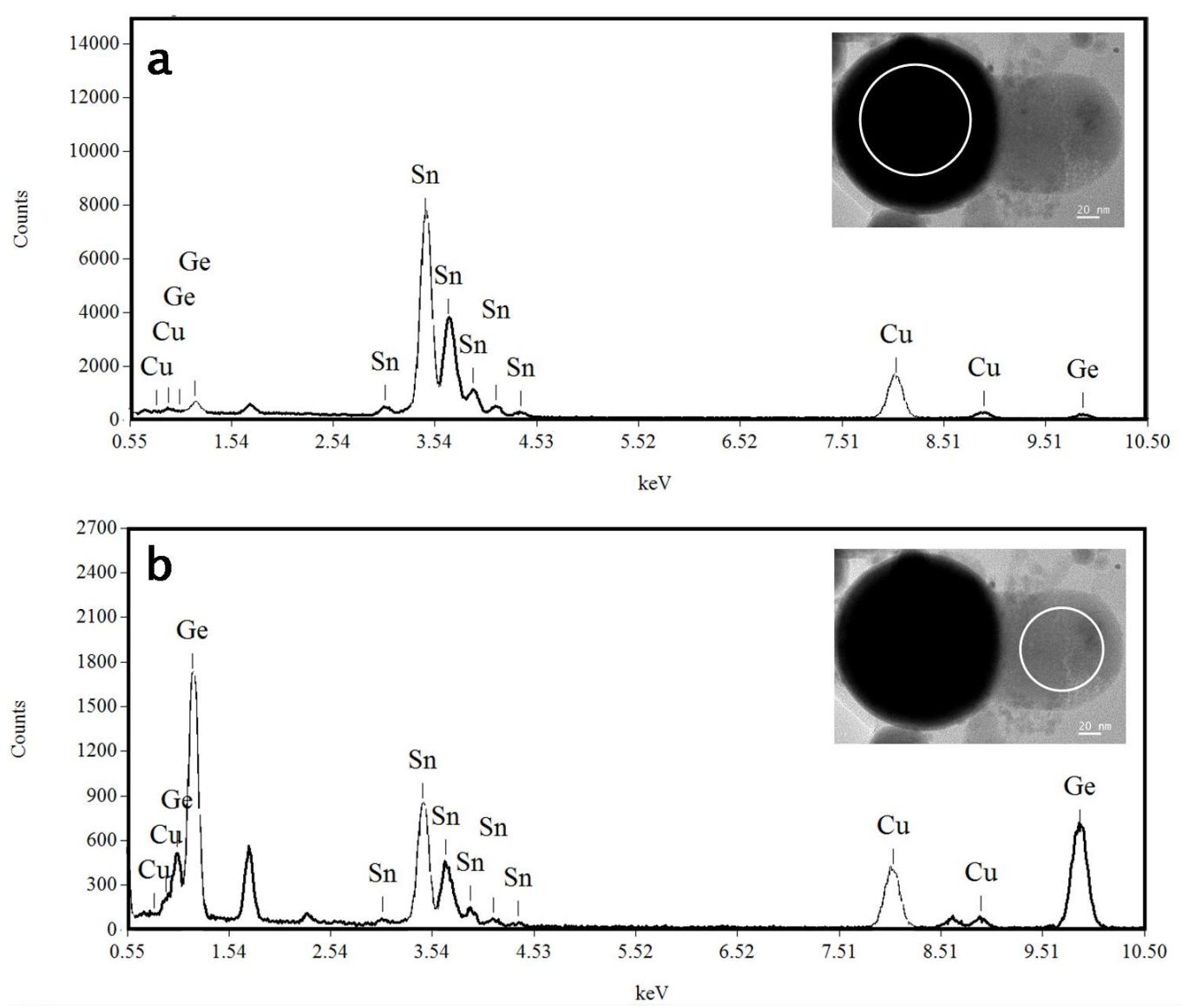

Fig. 4.5 EDX spectra of a tin germanium nanowire: the white circle drawn in the TEM images in the insets indicate the beam position. (a) EDX of the nanowire seed region. (b) EDX taken from the nanowire core. 
In summary, TEM and EDX results have provided evidence that this approach has some promise. Tin germanium nanowires can be prepared through SLS growth. But, to make straight and long tin germanium nanowires, the reaction conditions have to be modified.

\subsubsection{Experiment C: Effect of injection rate}

Previous studies have demonstrated that dropwise addition of the precursor tends to produce relatively longer nanowires, for example, growing silver nanowires on $\mathrm{Pt}$ or $\mathrm{Ag}$ nanoparticle seeds ${ }^{34}$ Hence, in this experiment, $\mathrm{Ge}(\mathrm{Ph})_{3} \mathrm{Cl}$ was injected dropwise at a rate of $\sim 0.005 \mathrm{~mL} / 5 \mathrm{~s}$. The other reaction conditions were kept as same as in experiment B.

\section{Results and discussion}

A typical TEM image of the nanowires prepared by dropwise injection of Ge precursor at $300{ }^{\circ} \mathrm{C}$ was shown in Fig. 4.6a. It was observed that the nanowires produced were curved and twisted. The length of the nanowires was around $200 \pm 80 \mathrm{~nm}$ and the average diameter was about $50 \pm 20$ nm.(Fig. 4.6 b,c)
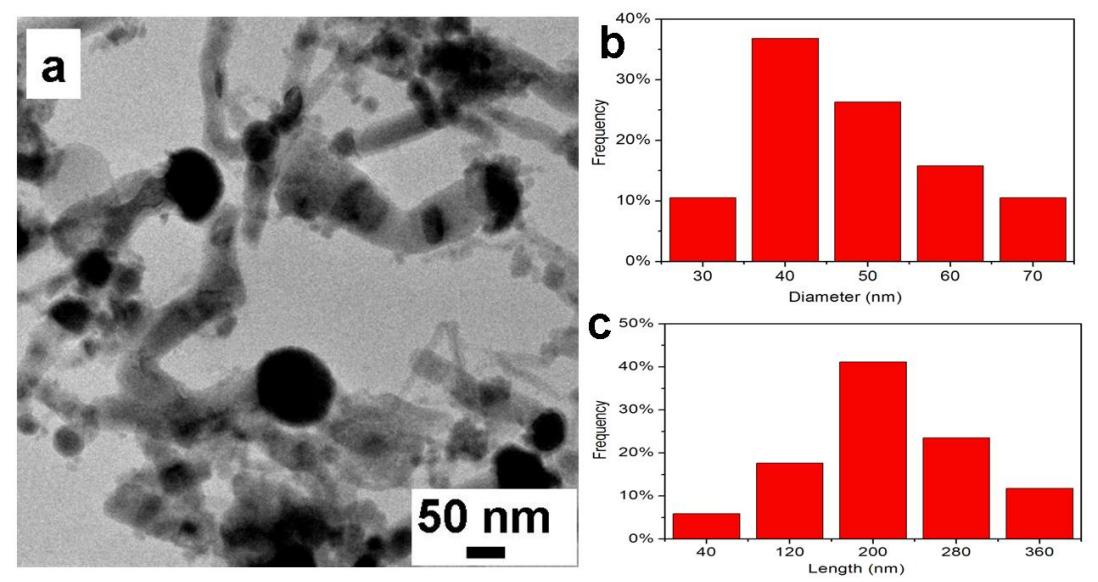

Fig. 4.6 (a) A typical LRTEM image of the product synthesized at $300{ }^{\circ} \mathrm{C}$ via dropwise injection; (b) and (c) The corresponding diameter and length distribution profiles. 
Table 4.1 Summary of results for tin germanium nanowire obtained with different injection rate.

\begin{tabular}{|l|l|l|l|l|}
\hline \multirow{2}{*}{ Exp } & \multirow{2}{*}{ Injection rate } & \multicolumn{3}{|c|}{ Products } \\
\cline { 3 - 5 } & & Morphology & Diameter & Length \\
\hline B & $0.5 \mathrm{~mL} / \mathrm{s}$ & straight NWs & $50 \pm 20 \mathrm{~nm}$ & $130 \pm 40 \mathrm{~nm}$ \\
\hline C & $0.005 \mathrm{~mL} / 5 \mathrm{~s}$ & curved NWs & $50 \pm 20 \mathrm{~nm}$ & $200 \pm 80 \mathrm{~nm}$ \\
\hline
\end{tabular}

Table 4.1 summarizes the results of tin germanium nanowires obtained at $300{ }^{\circ} \mathrm{C}$ with different injection rates of Ge precursor. It was found that dropwise injection of Ge precursor led to form longer nanowires than that obtained from swift injection of Ge precursor. However the nanowires produced shows a curved shape which was caused by defects in the nanowires. ${ }^{35,36}$ These defects may change the electrical properties of the nanowires and then affect the future potential applications. ${ }^{35}$ Although short nanowires were produced by swift addition of Ge precursor, the nanowires were generally straight. Hence, to make straight nanowires, in the following experiments, Ge precursor was added through the swift injection model, but with different Sn/Ge precursor ratios.

\subsubsection{Experiment D: Effect of $\mathrm{Sn} / \mathrm{Ge}$ precursors ratio}

Higher Sn/Ge precursor molar ratios were expected to produce relatively longer nanowire according to the previous studies. ${ }^{29}$ Hence, in experiment $D$, the ratio of $\mathrm{Sn} / \mathrm{Ge}$ was set $1: 30$ rather than $1: 10$ in experiment $B$, but the rest of the experiment parameters were kept the same.

\section{Results and discussion}

Fig. 4.7a shows a typical SEM image of the nanowires prepared at a ratio of $\mathrm{Sn} / \mathrm{Ge}$ precursors of 1:30. It was observed that as-prepared nanowires possess straight and smooth morphology. The diameter of the nanowires was ranging from $150 \pm 70 \mathrm{~nm}$ and the length reached $1.6 \pm 1.0 \mu \mathrm{m}$ (Fig. 4.7b,c). How- 
ever, the yield of the nanowire was low $(\sim 20 \%)$ and a lot of isolated bright big nanoparticles ( $>150 \mathrm{~nm}$ ) were observed in the sample. It is known that a region with higher atomic number elements appears brighter than lower atomic number elements in the backscattered electrons image. ${ }^{37}$ Hence, in Fig. 4.7a, the bright nanoparticles were expected to be Sn nanoparticles as element $\mathrm{Sn}$ (atomic number 50) has higher atomic number than element Ge (atomic number 32).
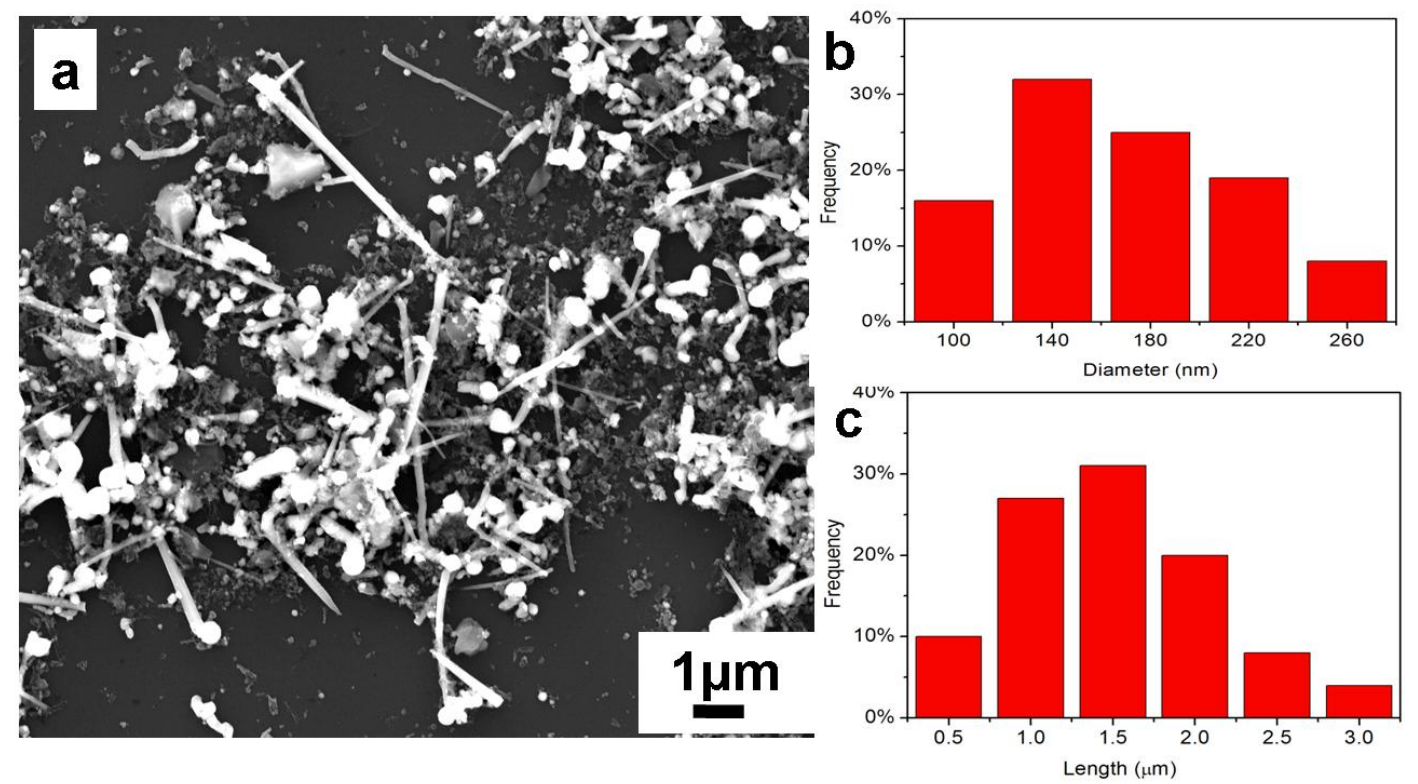

Fig. 4.7 (a) A SEM image of the product synthesized at $300{ }^{\circ} \mathrm{C}$ at molar ratio of Sn to Ge of 1:30; (b) and (c) The corresponding diameter and length distribution profiles.

Table 4.2 Summary of results for tin germanium nanowire obtained with different $\mathrm{Sn} / \mathrm{Ge}$ precursor ratio.

\begin{tabular}{|c|c|c|c|c|}
\hline \multirow{2}{*}{ Exp } & \multirow{2}{*}{$\begin{array}{l}\text { Sn/Ge precursors } \\
\text { ratio }\end{array}$} & \multicolumn{3}{|c|}{ Products } \\
\hline & & Morphology & Diameter & Length \\
\hline B & $1: 10$ & straight NWs & $50 \pm 20 \mathrm{~nm}$ & $130 \pm 40 \mathrm{~nm}$ \\
\hline $\mathrm{D}$ & $1: 30$ & straight NWs & $150 \pm 70 \mathrm{~nm}$ & $1.6 \pm 1.0 \mu \mathrm{m}$ \\
\hline
\end{tabular}

Table 4.2 summarizes the results of tin germanium nanowire obtained at different molar ratio of $\mathrm{Sn} / \mathrm{Ge}$ precursors. It was found that the molar ratio of $\mathrm{Sn} / \mathrm{Ge}$ precursors can significantly affect the length of the nanowire. The length of as- 
prepared nanowires can reach to micrometer size by varying the ratio of $\mathrm{Sn} / \mathrm{Ge}$ from $1: 10$ to $1: 30$.

In addition, experimental results show that small nanoparticles favor the growth of nanowires more than large nanoparticles. This can be explained by the SLS growth mechanism. ${ }^{27}$ During the synthesis, $\mathrm{Ge}^{0}$ was obtained from the reduction of $\mathrm{Ge}^{4+}$ and then diffused and dissolved into the liquid Sn droplets (reaction temperature $300{ }^{\circ} \mathrm{C}>$ melting point of Sn). After supersaturation is reached, nucleation and growth of tin germanium nanowire occurs. Small Sn nanoparticles were relatively easy to reach supersaturation concentration than large particles and consequently were easier to grow as longer nanowires.

In order to produce a high yield of smaller diameter tin germanium nanowires, small Sn seed nanoparticles were highly desirable. According to the results obtained in Chapter 3, it is known that the tin precursors play a crucial role in the tin nanoparticles size and size distribution control. Relatively small and narrow size distribution $\mathrm{Sn}$ nanoparticles were prepared by using $\mathrm{SnI}_{4}$ as the tin precursor rather than $\mathrm{SnCl}_{2}$. Hence, in the following experiments, $\mathrm{SnI}_{4}$ was used as a tin precursor.

\subsubsection{Experiment E: Effect of the size of tin nanoparticle}

The Sn nanoparticles used in experiment E were prepared in advance, under the optimal reaction conditions $\left(\mathrm{SnI}_{4}\right.$ was reduced at $200{ }^{\circ} \mathrm{C}$ in TOA) reported in Chapter 3. The average size of the Sn nanoparticles was $26.0 \pm 5.5 \mathrm{~nm}$. The remaining reaction conditions were same as in the experiment $\mathrm{D}$. 


\section{Results and discussion}

Fig. 4.8a shows a typical SEM image of as-prepared nanowires. It was observed that more than $80 \%$ of the product are nanowires. The as-prepared nanowires are generally straight with smooth surfaces. The average nanowire length is $4.5 \pm 2.0 \mu \mathrm{m}$, a few of the nanowires are longer than $5 \mu \mathrm{m}$. The diameter of the nanowires (measured at the half length of the nanowire) is about $70 \pm 40 \mathrm{~nm}$. In addition, it was noticed that the as-prepared nanowires have tapered ends (inset,Fig. 4.8a). The taper end away from the seed nanoparticle indicates that it was formed at the early stage of nanowire growth based on SLS mechanism. ${ }^{38}$ The diameter of the tapered end is about $30 \mathrm{~nm}$ which is close to the size of starting Sn seed nanoparticles. While in the other end of the nanowire (close to the seed particle region), the diameter of nanowire can reach to $\sim 100 \mathrm{~nm}$. It is known that $\mathrm{Sn}$ nanoparticles are difficult to stabilize and aggregate quickly at high temperature which will result in an enhanced particle size for seeds during the synthesis. This may result in the formation of a nanowire with increased diameter along the direction of growth of nanowire. Similar observation has been reported in a previous study. ${ }^{29}$ Another possible reason of the tapered end could be that at the start of reaction the concentration of Ge in the Sn nanoparticles is low which could produce the thin tapered end. When the reaction progresses, more Ge dissolves in the Sn seed and therefore the Ge nanowire diameter become thicker. 

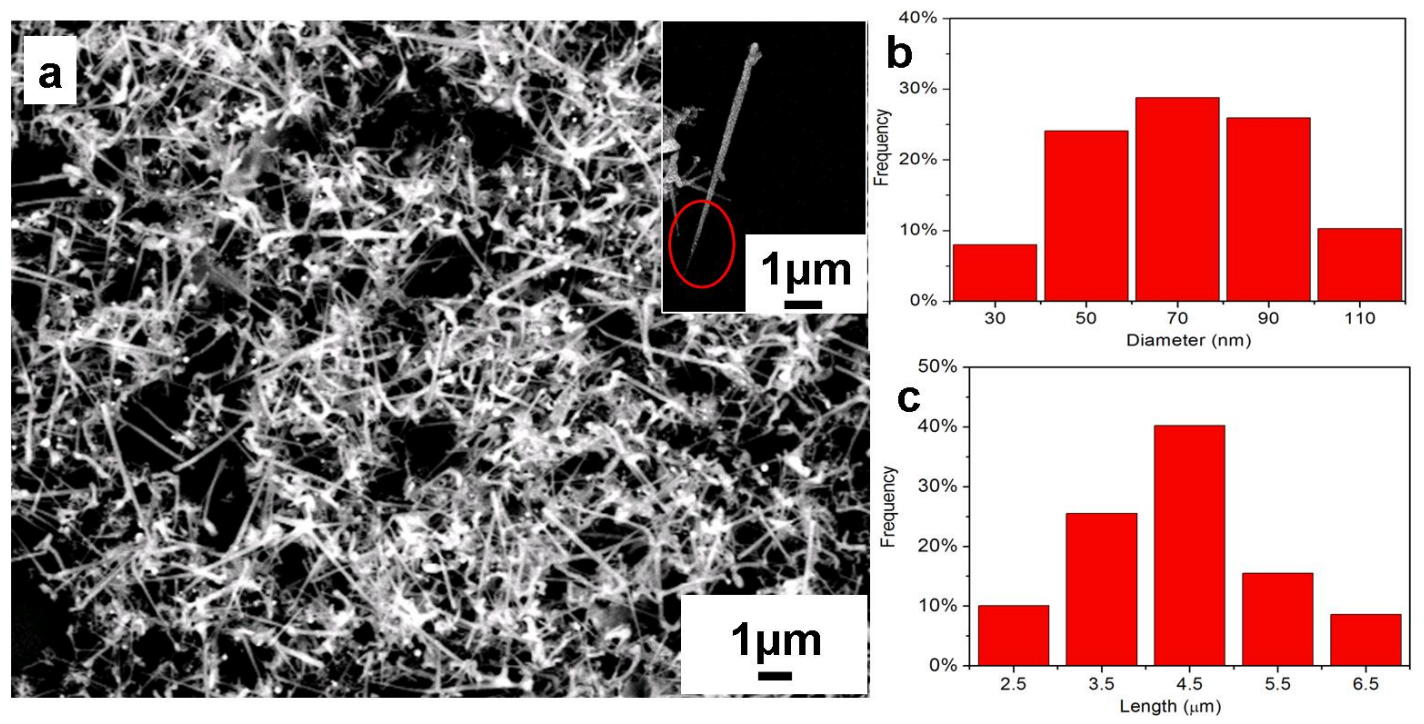

Fig. 4.8 (a) A SEM image of the product synthesized at $300{ }^{\circ} \mathrm{C}$ using preproduced seeds at molar ratio of $S n$ to Ge of 1:30, inset shows a single nanowire with tapered end. (b)and (c) The corresponding diameter and length distribution profiles.

Table 4.3 Summary of results for tin germanium nanowire obtained using different size Sn nanoparticles.

\begin{tabular}{|l|l|l|l|l|}
\hline \multirow{2}{*}{ Exp } & \multirow{2}{*}{ Sn NPs Size $(\mathrm{nm})$} & \multicolumn{3}{|c|}{ Products } \\
\cline { 3 - 5 } & & Morphology & Diameter & Length \\
\hline $\mathrm{D}$ & $45 \pm 20 \mathrm{~nm}$ & straight NWs & $150 \pm 70 \mathrm{~nm}$ & $1.6 \pm 1.0 \mu \mathrm{m}$ \\
\hline $\mathrm{E}$ & $26 \pm 5.5 \mathrm{~nm}$ & $\begin{array}{l}\text { straight NWs } \\
\text { taper end }\end{array}$ & $70 \pm 40 \mathrm{~nm}$ & $4.5 \pm 2.0 \mu \mathrm{m}$ \\
\hline
\end{tabular}

Table 4.3 summarizes the results of tin germanium nanowire obtained using different size Sn nanoparticles. High yield and longer nanowires were produced using relatively smaller size Sn seeds. Comparing the results from experiment D and E, it was found that the diameter of seed nanoparticles significantly affects the average diameter of the nanowires. This is in agreement with the SLS growth mechanism and is consistent with previous experimental results. ${ }^{28,39}$ 


\subsubsection{Experiment F: Effect of Sn nanoparticles made in situ or in advance}

According to the results presented in Chapter 3, Sn nanoparticles prepared at $300{ }^{\circ} \mathrm{C}$ possess similar size and slighter broader size distribution as that prepared under optimal reaction condition of $200{ }^{\circ} \mathrm{C}$. In order to develop a facile synthetic approach, in experiment F, Sn nanoparticles were prepare in situ by reducing $\mathrm{SnI}_{4}$ with $\mathrm{NaBH}_{4}$ in TOA at $300{ }^{\circ} \mathrm{C}$ then the Ge precursor was added and further reduced by $\mathrm{NaBH}_{4}$. The other reaction conditions were kept same as in experiment E.

\section{Results and discussion}

A typical SEM image of as-prepared nanowires is shown in Fig. 4.9. It was observed that $\sim 60 \%$ of the product are nanowires and the rest of them are nanoparticles. The nanowires were generally straight and several microns long (average length in $4.5 \pm 2.0 \mu \mathrm{m})$. Some of them $(\sim 10 \%)$ are longer than $8 \mu \mathrm{m}$. The diameter of the nanowire varies along their length, about $70 \pm$ $20 \mathrm{~nm}$ in the middle and tens of nanometers at their tip, producing both tapered ends(Fig. 4.10a). The formation of a tapered end away from the seed nanoparticles has been observed and discussed above (experiment E). The appearance of the second tapered end close to the seed nanoparticle may be due to the variation of the concentration of $\mathrm{Ge}^{0}$ in the solution over time. When the concentration of $\mathrm{Ge}^{0}$ in solution decreased after the consumption of $\mathrm{Ge}^{0}$ for nanowire growth, this could lead to a tapered end near the seed. 

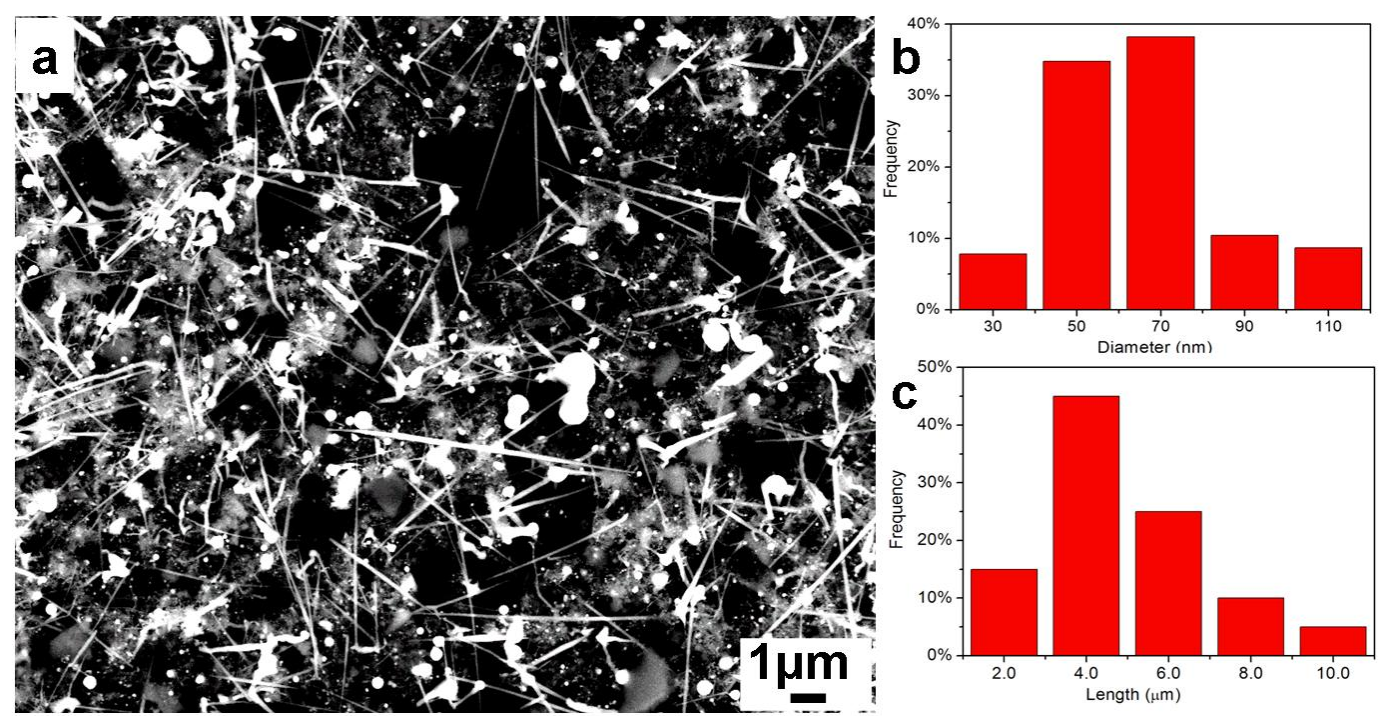

Fig. 4.9 (a) A SEM image of the product synthesized using $\mathrm{SnI}_{4}$ as the tin precursor; (b) and (c) the corresponding diameter and length distribution profiles.

Moreover, of one hundred nanowires examined, around $80 \%$ were found with Sn seeds at their tips while around $20 \%$ were observed without seeds (Fig. 4.10). This may due to the big difference in the surface free energy between Sn-rich tin germanium alloy seed and Ge-rich tin germanium alloy wire core, as well as the large lattice difference between them, resulting in surface segregation. ${ }^{19}$ As a consequence, seed nanoparticles may be separated from nanowires.
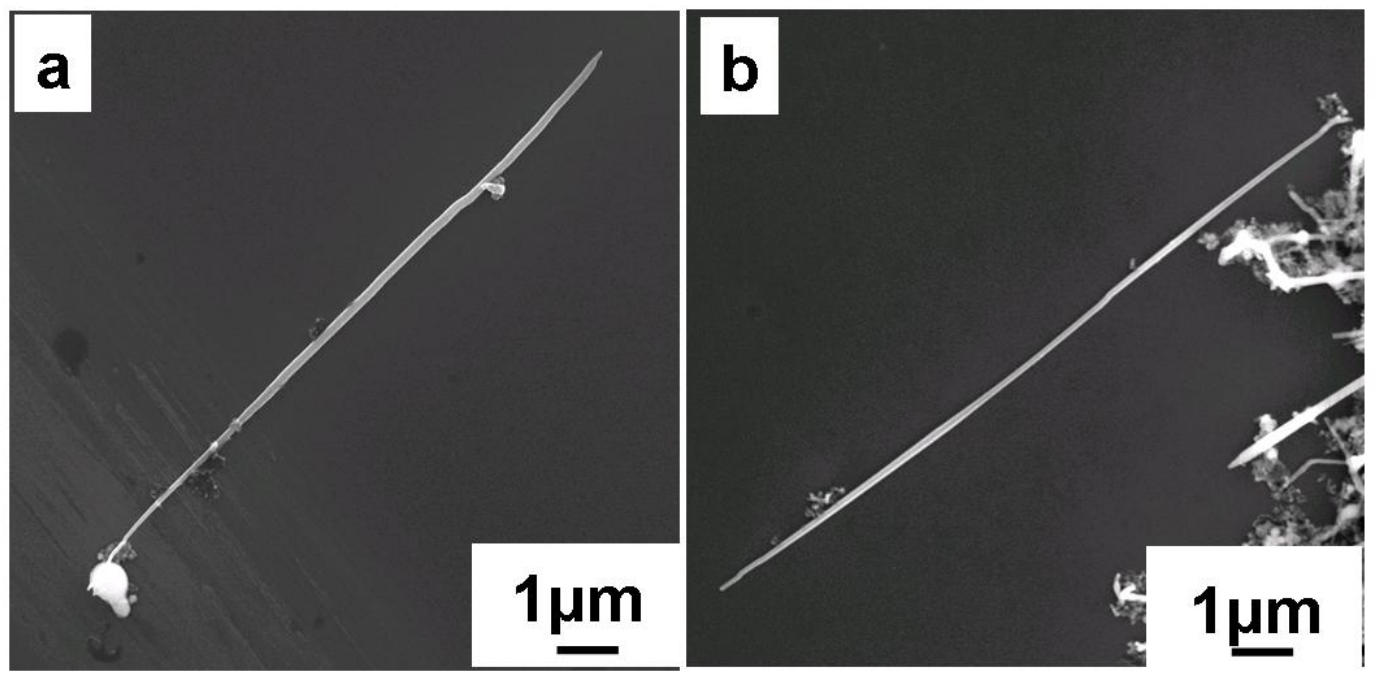

Fig. 4.10 Typical SEM images of nanowire with(a) or without (b) Sn seed particles at their tip. 
TEM characterization (Fig. 4.11a,b,c,) found some extremely short nanorods with Sn seeds in the sample. Fig. 4.11d,e presents the interface between seed nanoparticle and nanowire core. The observed crystal boundary was labeled with red dash line in Fig. 4.11d,e. This provides evidence for phase segregation.
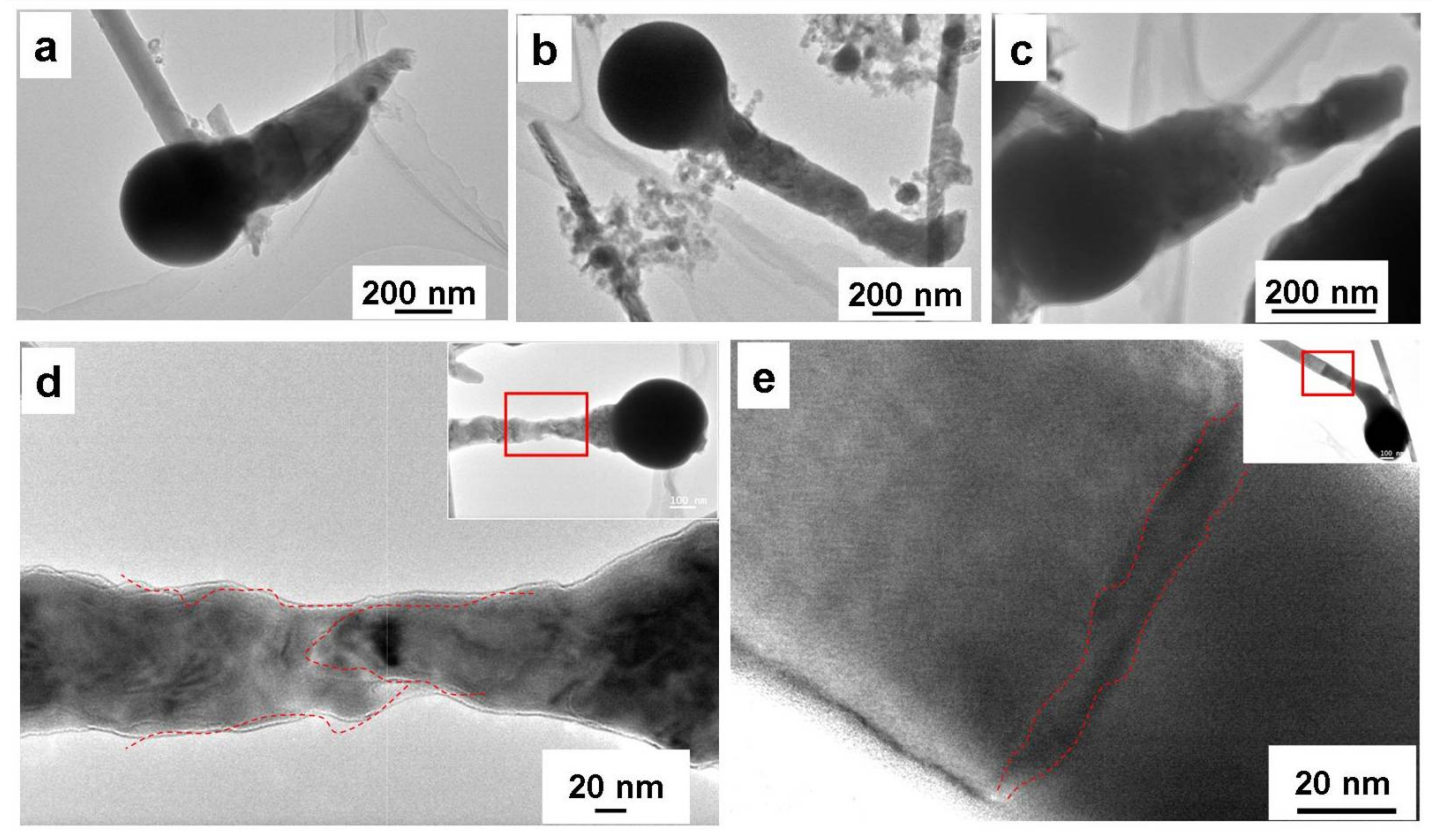

Fig. 4.11 LRTEM images of extremely short nanorods with Sn seed at their tips(a-c); HRTEM images of the transition region from seed to nanowires (labeled in the inset LRTEM images)(d-e).

Table 4.4 Summary of results for tin germanium nanowires obtained using different size Sn nanoparticles.

\begin{tabular}{|l|l|l|l|l|}
\hline \multirow{2}{*}{ Exp } & Sn NPs & \multicolumn{3}{|c|}{ Products } \\
\cline { 3 - 5 } E & $\begin{array}{l}26.0 \pm 5.5 \mathrm{~nm} \\
\text { seed in ad- } \\
\text { vance }\end{array}$ & $\begin{array}{l}\text { Straight NWs } \\
\text { tapered end for one } \\
\text { side }\end{array}$ & $70 \pm 40 \mathrm{~nm}$ & $4.5 \pm 2.0 \mu \mathrm{m}$ \\
\hline $\mathrm{F}$ & $\begin{array}{l}28.0 \pm 8.0 \mathrm{~nm} \\
\text { in situ seed }\end{array}$ & $\begin{array}{l}\text { straight NWs } \\
\text { tapered end for both } \\
\text { sides }\end{array}$ & $70 \pm 20 \mathrm{~nm}$ & $4.5 \pm 2.0 \mu \mathrm{m}$ \\
\hline
\end{tabular}

Table 4.4 summarizes the results of tin germanium nanowires synthesized using Sn nanoparticles prepared in advance or in stiu. Similar length and diam- 
eter of nanowires were produced in experiment $\mathrm{E}$ and $\mathrm{F}$ which indicates that nanowires could be prepared through one pot synthesis using in situ made Sn nanoparticles. However, the yield of nanowires was slightly lower in experiment $\mathrm{F}(60 \%)$ than that in experiment $\mathrm{E}(80 \%)$ which is probably due to two reasons:(1) some particles with extremely short rods formed after phase segregation. This may give some value for future potential application in which removing seed nanoparticle is generally required. (2) Small Sn nanoparticles tend to aggregate and form big Sn nanoparticles which is unfavorable for nanowire growth.

\subsubsection{Experiment G: Effect of volume of solvent}

Adding more solvent was expected to minimize the aggregation of Sn nanoparticles. In experiment $\mathrm{G}, 20 \mathrm{~mL}$ of TOA instead of $10 \mathrm{~mL}$ of TOA in experiment $\mathrm{F}$ was used. The other reaction parameters were same as that in experiment $\mathrm{F}$.

\section{Results and discussion}

Fig. 4.12 shows a SEM image of the nanowires synthesized in the presence of $20 \mathrm{~mL}$ TOA. It was found that $\sim 80 \%$ of the product are nanowires. The nanowires are straight and $60 \pm 20 \mathrm{~nm}$ in diameter, $5.5 \pm 2.0 \mu \mathrm{m}$ in length. More than $15 \%$ of the nanowires are longer than $8 \mu \mathrm{m}$. The aspect ratio of nanowire can exceed 100. Nanowires with both tapered ends and some without $\mathrm{Sn}$ seeds nanowires were also observed in the product. 

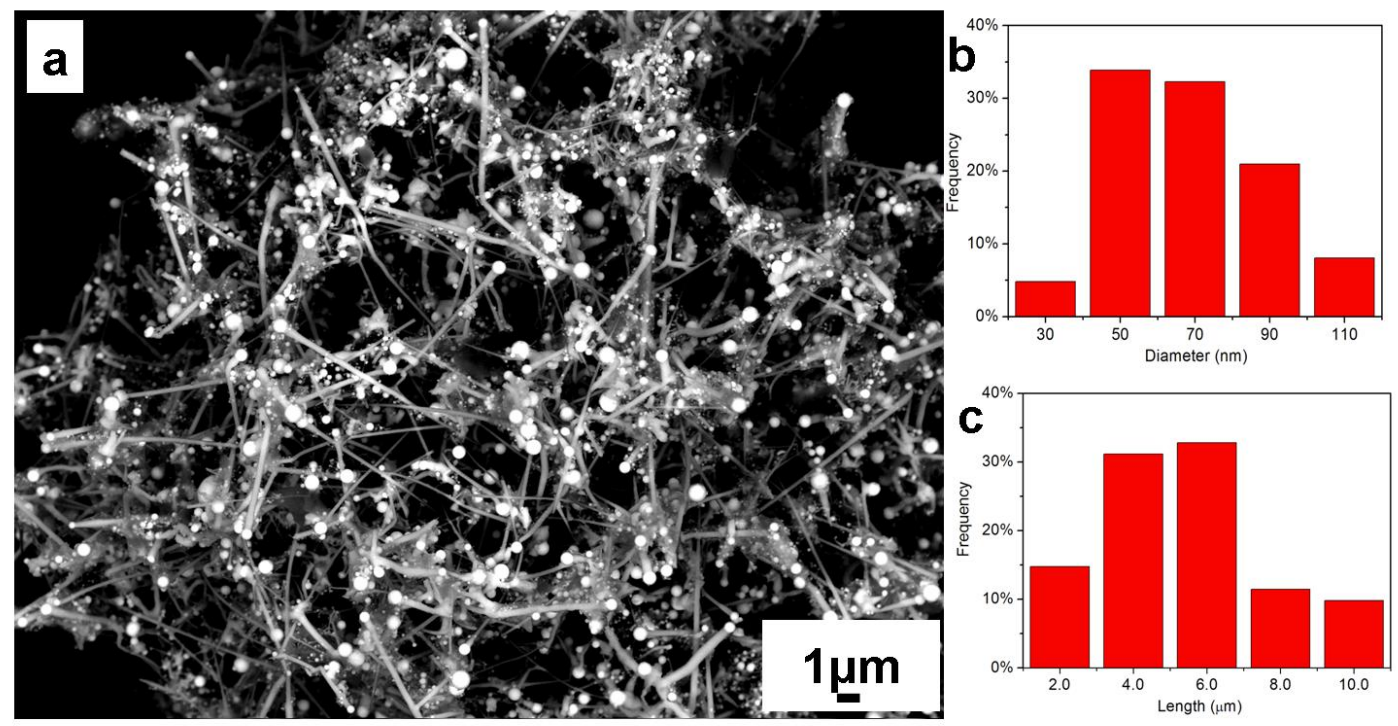

Fig. 4.12 (a) A SEM image of the product synthesized at $300{ }^{\circ} \mathrm{C}$ at molar ratio of $\mathrm{Sn}$ to Ge of 1:30 in $20 \mathrm{~mL}$ TOA; (b-c) The corresponding diameter and length distribution profiles.

Table 4.5 Summary of results for tin germanium nanowires obtained using different size Sn nanoparticles.

\begin{tabular}{|l|l|l|l|l|}
\hline \multirow{2}{*}{ Exp } & \multirow{2}{*}{ TOA $(\mathrm{mL})$} & \multicolumn{3}{|c|}{ Products } \\
\cline { 3 - 5 } & 10 & $\begin{array}{l}\text { Morphology } \\
\text { taper end for both sides } \\
\text { taight NWs }\end{array}$ & $70 \pm 20 \mathrm{~nm}$ & $4.5 \pm 2.0 \mu \mathrm{m}$ \\
\hline $\mathrm{G}$ & 20 & $\begin{array}{l}\text { straight NWs } \\
\text { taper end for one side }\end{array}$ & $60 \pm 20 \mathrm{~nm}$ & $5.5 \pm 2.0 \mu \mathrm{m}$ \\
\hline
\end{tabular}

Table 4.5 summarizes the results of tin germanium nanowires synthesized using different amount of TOA. It was found that high yield, relatively narrow size distribution and long nanowires can be synthesized by adding more surfactant.

In summary, based on the results obtained from this series of experiments, it was found that the morphology, length and diameter of the nanowires could be varied by changing experimental parameters. Reaction temperature plays an important role in the formation of nanowires. Nanowire growth is favored at high temperature. The length of nanowires was determined by the molar 
ratio of $\mathrm{Ge} / \mathrm{Sn}$ precursors. Longer nanowires will be produced under higher $\mathrm{Ge} / \mathrm{Sn}$ ratio. In addition, it was found that the size of Sn nanoparticles affects the yield and diameter of nanowires. Small particles favor the growth of nanowires. Moreover, similar quality nanowires can be synthesized through a facile one pot synthesis in which Sn nanoparticles were made in situ. Adding more solvent (i.e., lower precursor concentration) is good for nanowire size control.

The one pot synthesis with $\mathrm{Sn} / \mathrm{Ge}$ ratio of $1: 30$ at $300{ }^{\circ} \mathrm{C}$ in the presence of $20 \mathrm{~mL}$ TOA was found to be the optimal condition with respect to morphology, length, diameter and yield. Since tapered nanowire ends may affect future optoelectronic applications, it could be solved in future work by adding a stronger surfactant, changing the reaction time or using a different Ge precursor.

\subsection{Composition Analysis of tin germanium nanowires}

The composition of the nanowires prepared under optimal condition (experiment G) was investigated. XRD measurement was used to analyse a large amount of nanowires. EDX/SEM and EDX/STEM were used to study individual nanowires. The measurement results were presented in the following subsection:

\subsubsection{XRD measurement}

Fig. 4.13 shows a typical XRD spectra for the sample. Three diffraction peaks were indexed and labeled as (111), (220) and (311) of the crystalline diamond structure of $\mathrm{Sn}_{x} \mathrm{Ge}_{1-x}$. The scattering angle $2 \theta$ of these peaks showed a shift to lower scattering angles, compared to pure Ge (space group Fd3m, a=5.658 $)$ ). 
The corresponding d values were accurately determined by employing Bragg's law and using the underlying single crystal silicon substrate, oriented in $\{111\}$ planes (space group Fd3m, a=5.4309 $\AA$ ), as a standard. The lattice parameter a of the $\mathrm{Sn}_{x} \mathrm{Ge}_{1-x}$ nanowires was calculated from the $\mathrm{d}$ values for the peaks, based on the method described in Chapter 2, and the result was presented in Table 4.6. The composition of the $\mathrm{Sn}_{x} \mathrm{Ge}_{1-x}$ nanowires was determined as $\mathrm{Sn}_{0.1} \mathrm{Ge}_{0.9}$.

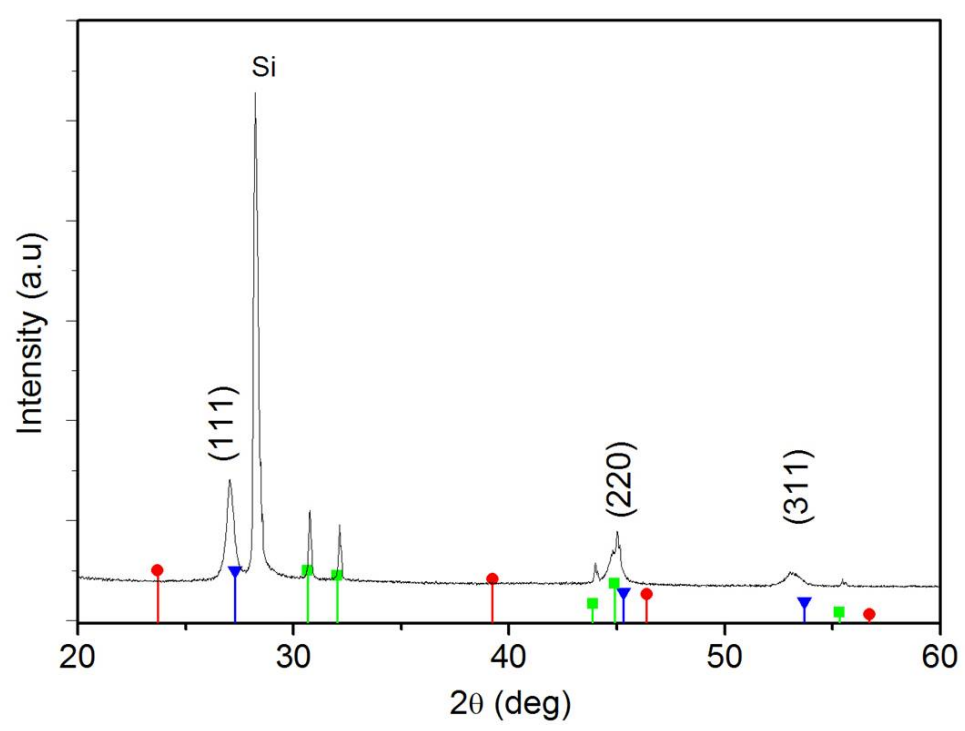

Fig. 4.13 A XRD pattern of the nanowire synthesized at an optimal reaction condition ( $\mathrm{Cu}$ radiation). The expected positions and intensities for peaks corresponding to cubic Sn (ICDD 00-005-039), cubic Ge (ICDD 00-004-0545) and tetragonal Sn (ICDD 00-004-0673) are marked by lines labeled with "•" " " " ", respectively.

Table 4.6 Lattice parameter a calculated from XRD pattern.

\begin{tabular}{|c|c|c|c|}
\hline $\mathrm{h} \mathrm{k} \mathrm{l}$ & 111 & 220 & 311 \\
\hline $\mathrm{a}$ & 5.739 & 5.745 & 5.743 \\
\hline $\mathrm{a}_{\text {mean }}$ & \multicolumn{3}{|c|}{$5.742 \pm 0.003$} \\
\hline
\end{tabular}

Moreover, XRD also revealed the presence of tetragonal phase Sn. The diffraction angle of peaks were slightly shifted to high scattering angle indicating a slight distortion of the tetragonal cell of $\beta$-Sn. The lattice distortion was further detected and confirmed in the following local crystal structure investigation 
section. No detectable XRD peaks corresponding to cubic phase $\alpha$-Sn were observed.

\subsubsection{EDX/SEM and EDX/STEM characterization}

\section{EDX/SEM characterization}

EDX/SEM line scanning and elemental mapping mode were utilized to determine the elemental distribution in the nanowire, as shown in Fig. 4.14. The SEM image (Fig. 4.14a) of an isolated nanowire reveals bright-dark imaging contrasts from the seed segment as compared to the wire segment while the associated EDX line-scan (Fig. 4.14b) reveals the Sn-rich seed segment and Gerich wire segment. EDX elemental mapping from the same nanowire (Fig. 4.14c,d) clearly shows the spatial distribution of Sn and Ge along the length of the nanowire. Both Ge and Sn were homogeneously distributed in the wire segment.
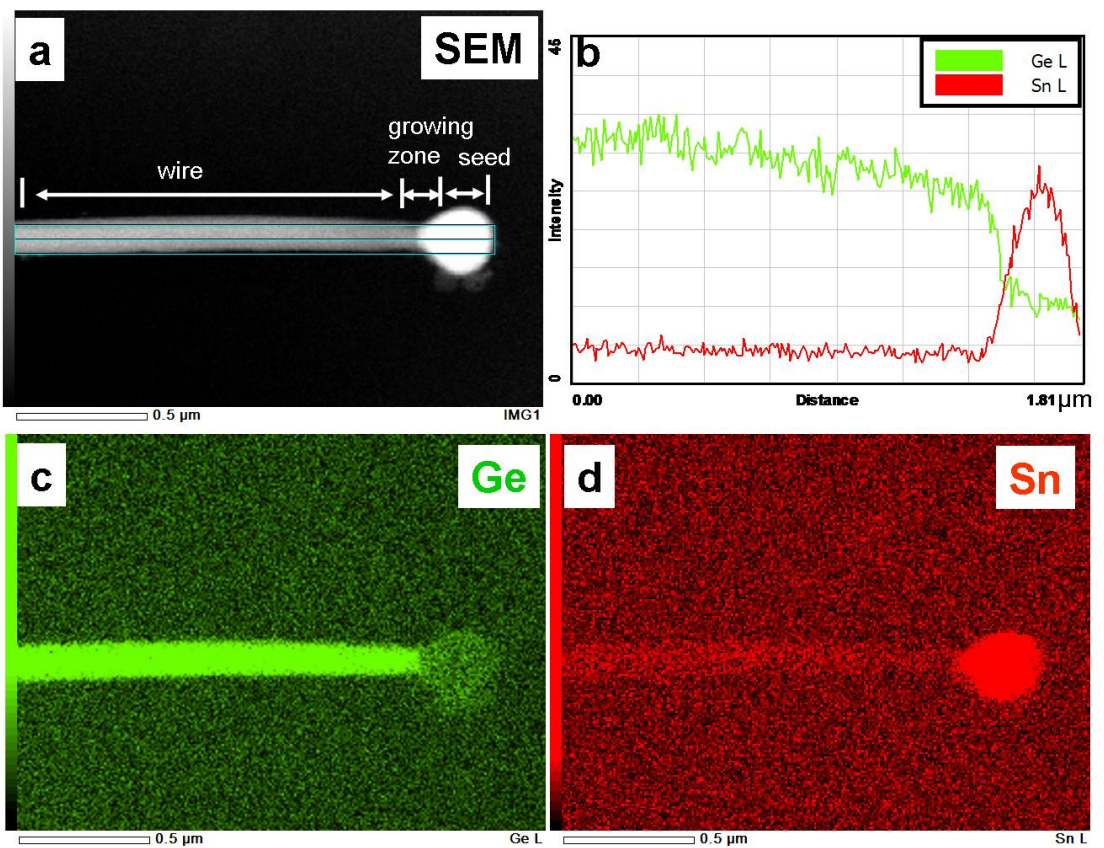

Fig. 4.14 A SEM image of a $\mathrm{Sn}_{1-x} \mathrm{Ge}_{x}$ nanowire (a) synthesized at $300{ }^{\circ} \mathrm{C}$ under a molar ratio of $\mathrm{Sn}$ to Ge of 1:30; compositional line profiles probed by SEM/EDX (b) along the blue line in (a). EDX element mapping of (c) the Sn L edge, (d) Ge L edge. 


\section{EDX/STEM characterization}

EDX results presented in Fig. 4.14 shows a distinct variation in composition of Sn and Ge across the growing zone (the zone between the seed and wire). To extensively study the element distributions of Sn and Ge at the growing zone in the nano domain,STEM combined with EDX was employed.

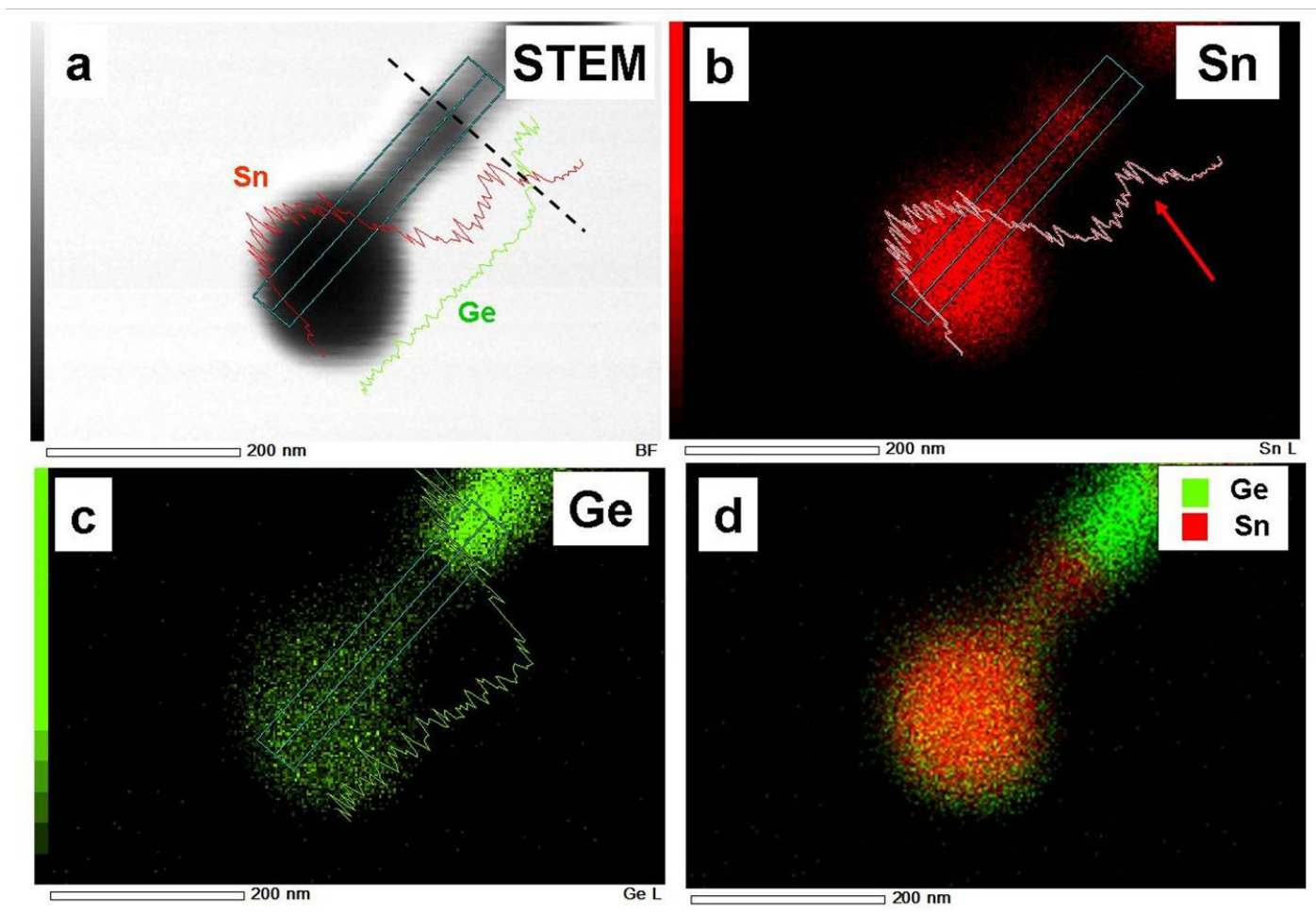

Fig. 4.15 A bright field STEM image of a $\mathrm{Sn}_{1-x} \mathrm{Ge}_{x}$ nanowire (a) synthesized at $300{ }^{\circ} \mathrm{C}$ under a molar ratio of $\mathrm{Sn}$ to $\mathrm{Ge}$ of 1:30; The spatial distribution of each element is shown in different colour (b) Sn, (c) Ge, and (d) overall reconstructed elemental distribution.

Fig. 4.15a shows an EDX line-scan correlated with the physical position of the growing zone of a nanowire. The line scan revealed that the seed segment consisted of a larger amount of Sn with small amount of Ge. More significantly, an additional Sn peak (labeled in Fig. 4.15b) was found at the interface of the nanowire which means the content of $\mathrm{Sn}$ at that position is relatively high. It is well known that $\mathrm{Sn}$ has a lower surface free energy than Ge as well as the limited solid solubility between Sn and Ge. Therefore, Sn tends to segregate 
to the Sn-rich seed segment and subsequently form a relatively high content at the interface. This may lead to the separation of the seed nanoparticle from the wire core.

Fig. 4.15c shows that the elemental Ge is uniformly distributed in the seed segment. It was also observed that Ge (green spots) cover the surface of the Sn seeds (red spots) (Fig. 4.15d) which indicates the nanowire growth took place through the SLS model. ${ }^{39}$

In summary, XRD and EDX measurement indicate that the composition of Sn in as-prepared $\mathrm{Sn}_{x} \mathrm{Ge}_{1-x}$ nanowires (under optimal reaction condition) is 0.1 which is equal to the critical ratio obtained from previous studies. Above this ratio, $\mathrm{Sn}_{x} \mathrm{Ge}_{1-x}$ will exhibit a direct band gap while below than that, it will possess an indirect band gap. ${ }^{14}$

\subsection{Local Structure Characterization of Nanowires}

High resolution transmission electron microscopy (HRTEM) and selected area electron diffraction (SAED) were used to probe the crystallographic details, including the growth direction and growing zone crystal structure.

\subsubsection{Growth direction analysis}

Fig. 4.16 and Fig. 4.17 show typical HRTEM images of the tin germanium nanowires synthesized under the optimal conditions as in the experiment G. HRTEM image and SAED pattern reveals that the nanowires have face-centered cubic crystal structure, which is consistent with the result obtained from XRD measurement (section 4.3.1). The TEM images in Fig. 4.16 of individual nanowires show clear lattice fringes and indicate the single crystal nature of the nanowires. 
The inset SAED patterns in Fig. 4.16a and Fig. 4.16b were recorded with electron beam along [01] and [111], respectively. From the SAED patterns, the growth direction of these nanowires were indexed as [111] and [110], respectively.

Fig. 4.17a shows an individual nanowire with a [112] growth direction which was confirmed by the corresponding SAED pattern (zone axis: [101] , Fig. 4.17b). The SAED pattern clearly shows twin diffraction reflections, which have been indexed in Fig. 4.17b, indicating the formation of longitudinal $\{111\}$ twins. The twin boundary extends parallel to the growth direction.

Of the twenty nanowires examined, around $70 \%$ exhibited single crystal structures with either $<110>(\approx 85 \%)$ or $<111>(\approx 15 \%)$ growth direction and the remaining fraction were twinned nanowires with $<112>$ growth direction. These results agree with previous research in which the nanowire with diamond structure such as Ge, Si, grow predominantly along the $<110>$ and $<111>$ directions. ${ }^{29,40,41}$ Some $<112>$ growth twins has also been observed. ${ }^{42}$ Based on the research in Si and Ge nanowires, it was considered that the preferred growth direction are $<111>$ for larger diameter nanowire and $<110>$ and $<112>$ for smaller diameter. ${ }^{43}$ In fact, in the current research, the growth direction for nanowire is seemingly independent of the nanowire size. In addition, Samuelson and coworkers suggested high supersaturation and high temperature conditions leading to twins. ${ }^{44}$ Hidalgo et al. reported that trace amounts of $\mathrm{Sn}$ in nanowire may influence the twin boundary energy of crystal, either causing an increase or a decrease depending on the host. ${ }^{45}$ In the current research, these may account for the formation of twinned nanowires. 

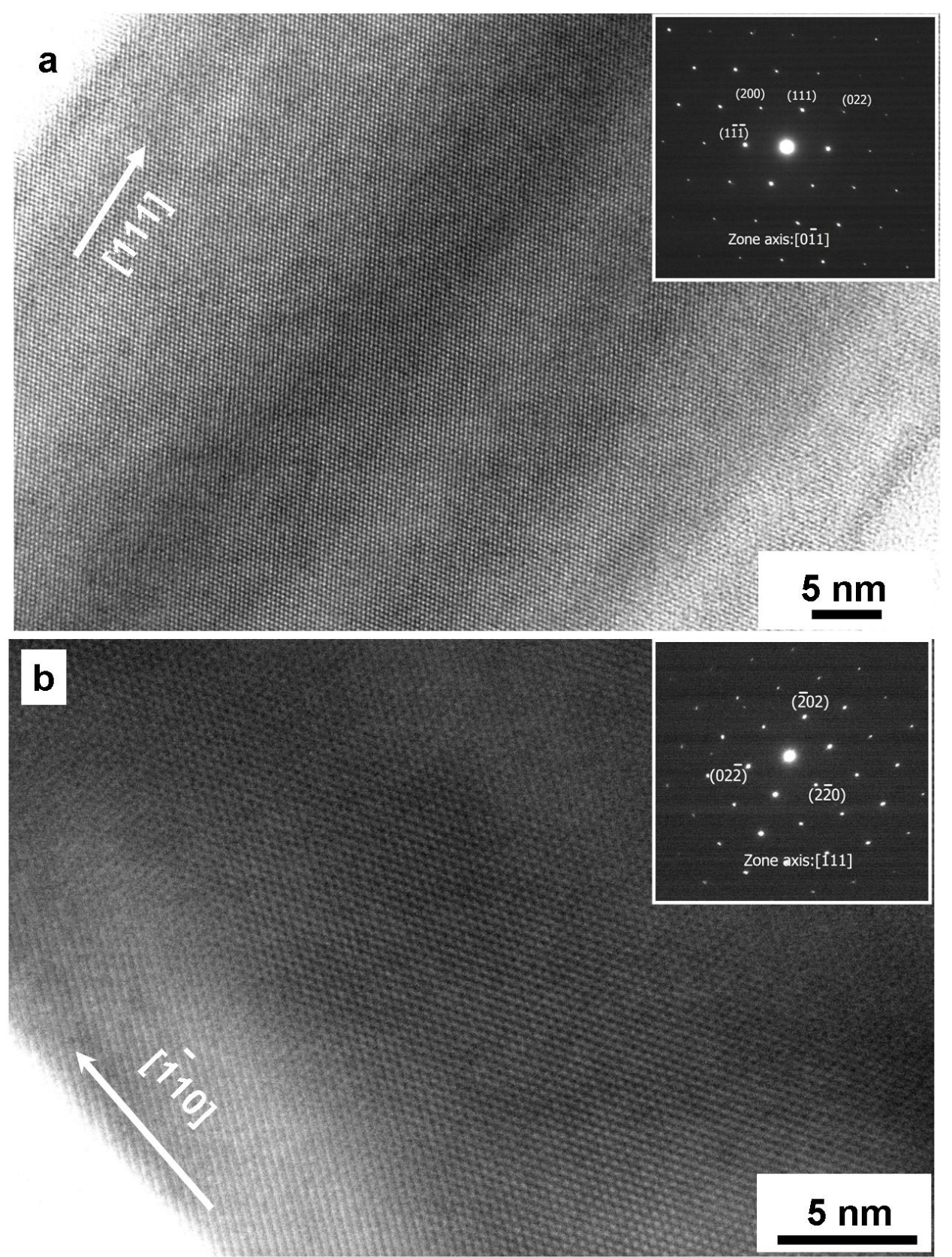

Fig. 4.16 (a) A HRTEM images of a nanowire with [111] growth direction taken along the [011] zone axis and a SAED pattern (inset). The forbidden spot of (200) is from double diffraction of (111) and (11)1); (b) A HRTEM image of a nanowire with [110] growth direction taken along the [111] zone axis and SAED pattern(inset). 

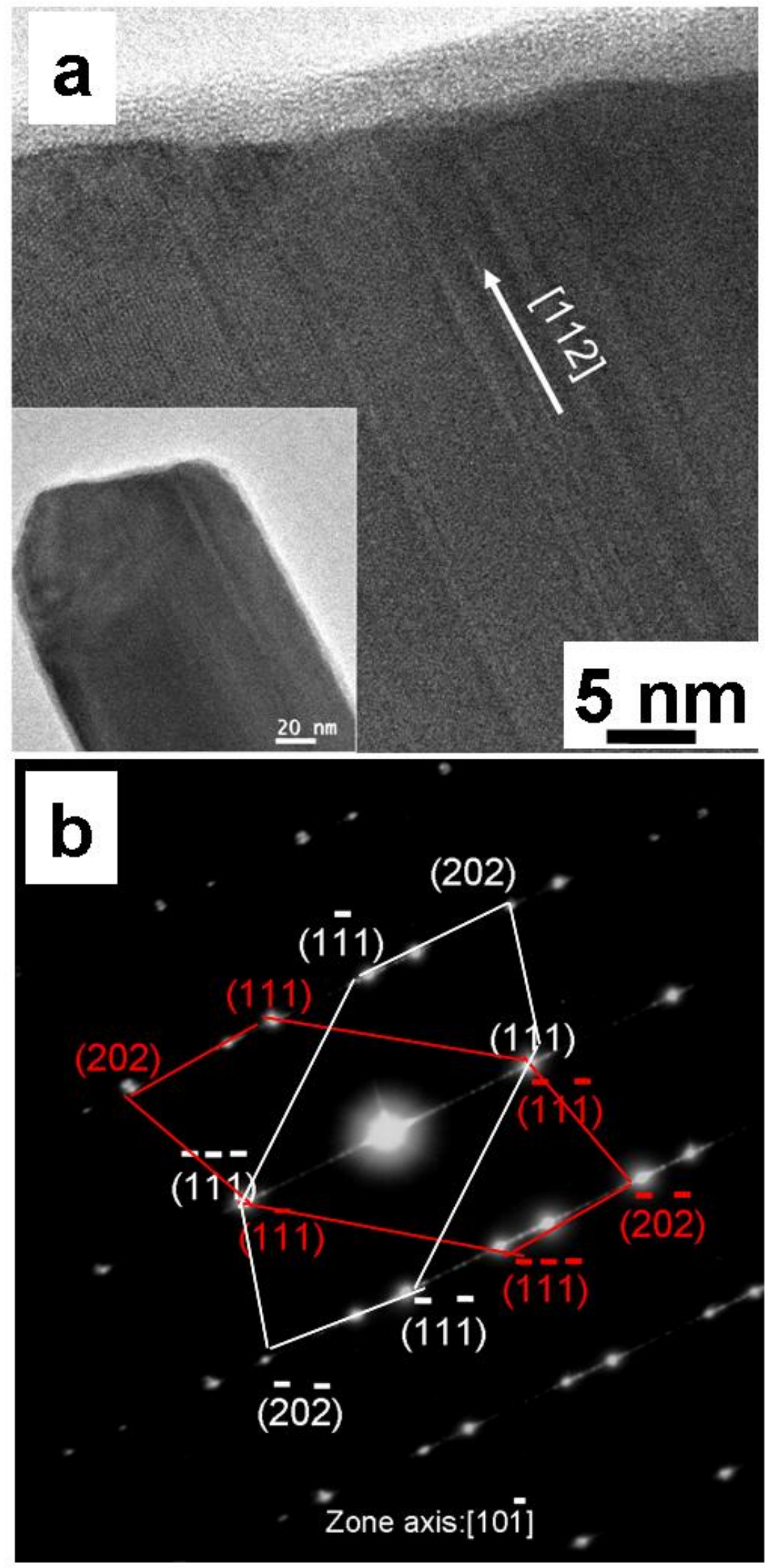

Fig. 4.17 (a) An individual nanowire growth along [112] direction with longitudinal $\{111\}$ twins HRTEM image (inset: LRTEM image of the nanowire); (b) The SAED pattern recorded along [10̄1]. 


\subsubsection{Growing Zone Crystal Structure Study}

Patriarche et al. proposed that the curvature of the interface between the seed nanoparticle and the nanowire affects the growth direction of nanowire. ${ }^{46} \mathrm{Ko}-$ rgel et al. modeled the relationship between the seed/nanowire contact angle and twin nucleation probability, demonstrating that contact angles $\geq 109^{\circ}$ lead to lamellar twins. ${ }^{40}$ Rogers and Fydell found that the presence of trace amounts of elemental Ge in tetragonal Sn, can accelerate the transformation from tetragonal phase Sn (stable $>13.2{ }^{\circ} \mathrm{C}$ ) to cubic phase Sn (stable $\left.<13.2{ }^{\circ} \mathrm{C}\right) .{ }^{47}$ All of these raises interest to extensively study the detailed crystal structure in the growing zone of the as-prepared tin germanium alloy nanowires. In this research, two types of growing zone with different external shape and interface were commonly observed (Scheme 4.1) and depicted as following:

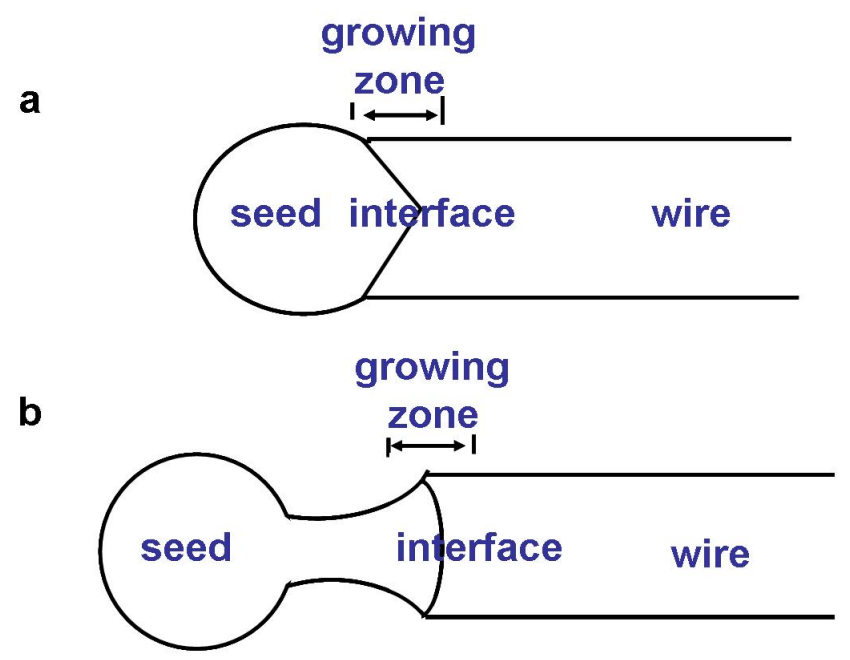

Scheme 4.1 Growing zone with curved interface (a) and a nearly flat interface (b).

\section{Growing zone with curved interface}

A typical HRTEM image of a growing zone with highly curved (V-shaped) interface of the seed nanoparticle/the nanowire is shown in Fig. 4.18 (the inset is the LRTEM image of the nanowire). Examination of the fast Fourier transfor- 
mation (FFT) of selected domains (domain $\mathrm{i}$ and ii in Fig. 4.18), revealed that the V-shaped interface consists of two $\{111\}$ planes. The measured d-spacing $(\sim 3.34 \AA)$ of the (111) lattice planes in both domain i and domain ii are larger than the d-spacing of (111) lattice planes of pure Ge $(\mathrm{d}=3.24 \AA)$ with cubic structure, confirming the presence of Sn atoms in Ge cubic cell (lattice parameter a of $\mathrm{Sn}_{x} \mathrm{Ge}_{1-x}>$ lattice parameter a of $\left.\mathrm{Ge}\right)$. The combination of (111) and (11) $)$ results in a growth axis of $<112>$. HRTEM characterization leads to the proposal that twin defects originated during nucleation. Then as the nanowire continues to grow, the $\{111\}$ twins extend down the length of the nanowires. A similar conclusion was also obtained by Korgel and Hanrath. ${ }^{36}$

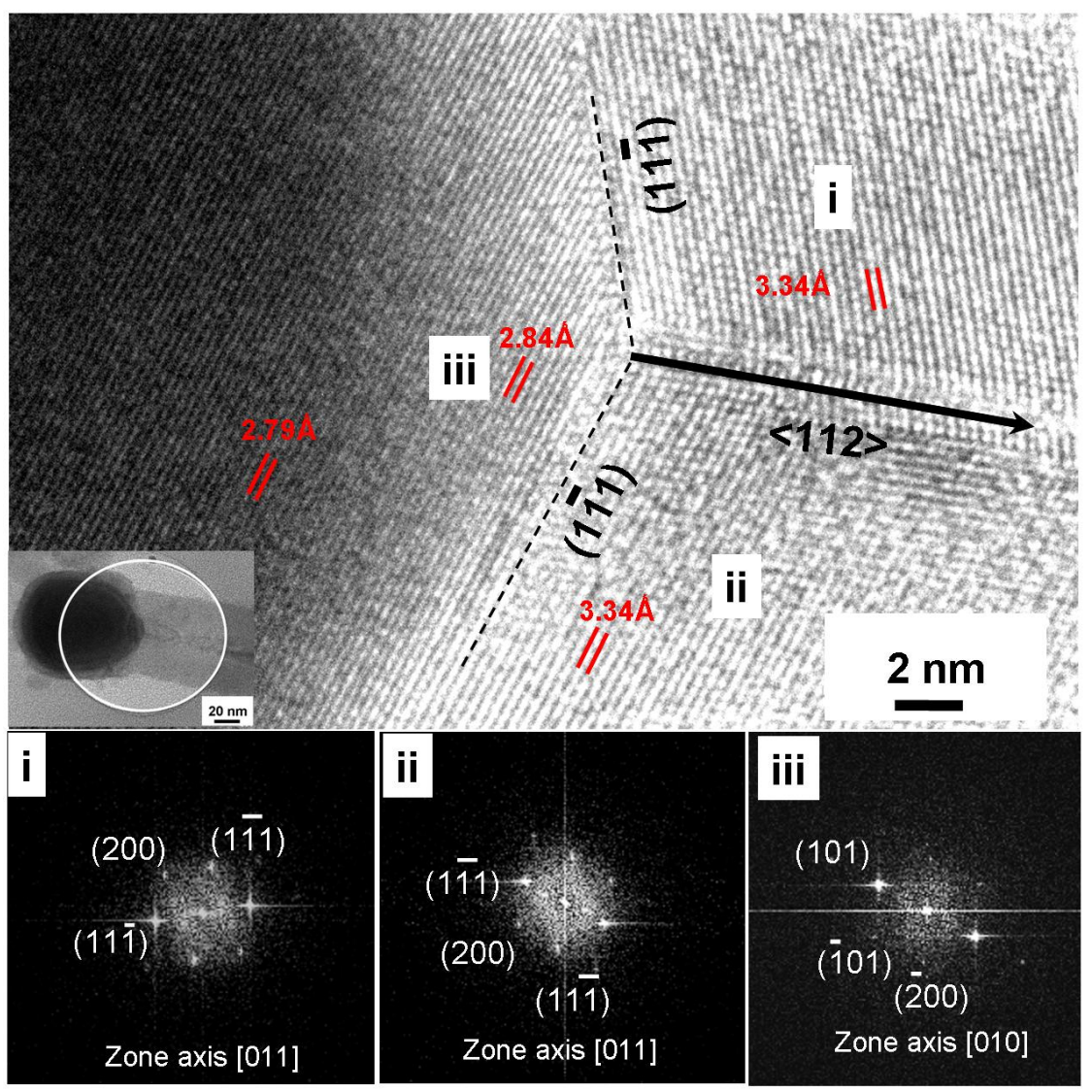

Fig. 4.18 A HRTEM image of a twining nanowire with V-shaped interface(inset: a LRTEM image of the nanowire) The corresponding fast Fourier transform (FFT) of (i), (ii) and (iii) shown in the HRTEM image

Moreover, clear lattice fringes as shown in the growing zone (domain iii, Fig. 4.18) 
revealed that the lattice spacing increases from $2.79 \AA$ (matching the d-spacing

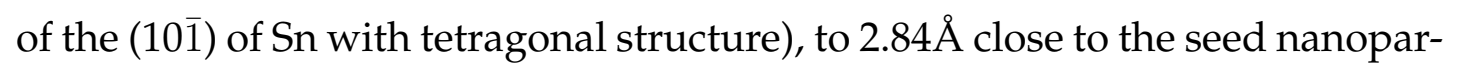
ticle/nanowire interface. FFT analysis in domain (iii) further confirmed the presence of a tetragonal crystal structure. Crystal defects such as dislocation, grain boundaries and stacking faults were observed in the growing zone.

\section{Growing zone with a flat interface}

Fig. 4.19a shows a LRTEM image of a single crystal nanowire with a growth orientation along the $<110>$ direction (confirmed by SAED pattern).

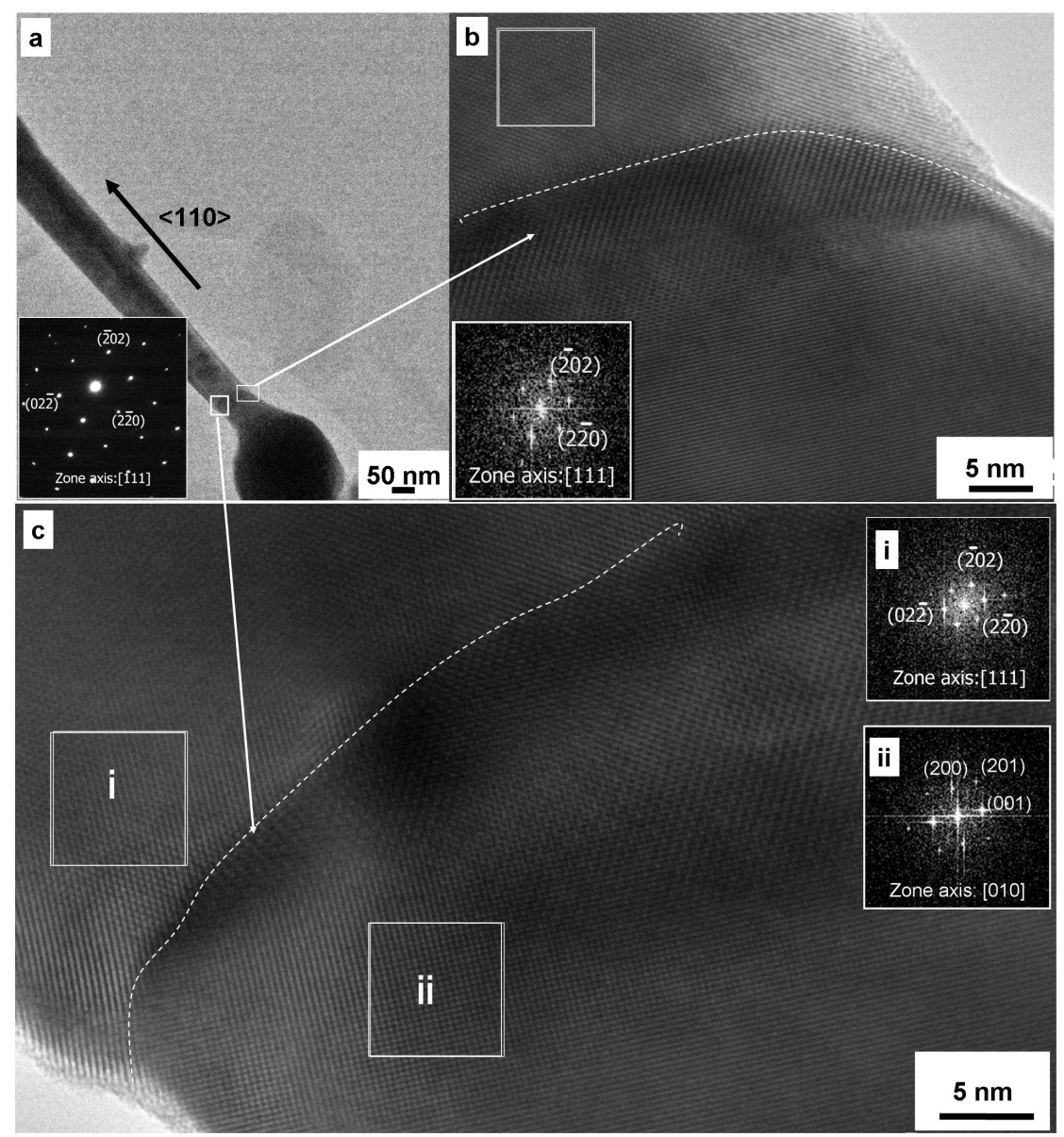

Fig. 4.19 (a) A LRTEM image of a nanowire with [110] growth direction taken along the [111] zone axis. (Inset: SAED patten) (b) and (c) HRTEM images of the growing zone of the nanowire. (Inset) FFT indicate different crystal structures in the wire and seed segments, individually. 
The growing zone of the nanowire was studied by HRTEM. The corresponding HRTEM images were presented in Fig. $4.19 \mathrm{~b}, \mathrm{c}$. A clear crystal boundary was observed at the interface (the seed/the wire) and marked with white dashed lines in Fig. 4.19b,c. Examination of the diffraction pattern generated by FFT of selected areas in the HRTEM images revealed that the crystal structure differs between the wire and seed segments. A face-centered cubic structure was found in the wire segment while a body-centered tetragonal structure was present in the seed segment (Fig. 4.19b,c). The larger lattice mismatch could result in the formation of the observed crystal boundary at the interface.

\subsection{Growth Mechanism of Nanowires}

This chapter demonstrated a self-catalyzed solution-liquid-solid (SLS) growth of tin germanium nanowires. The SLS growth mechanism, which was proposed by Buhro et al., ${ }^{39}$ is analogous to the vapor-liquid-solid (VLS) mechanism discovered by Wanger and Ellis in $1964 .{ }^{48}$ The general SLS growth mechanism has been described in detail in Chapter 1. Herein, the growth processes of tin germanium nanowire could be divided into four stages according to the SLS mechanism(Scheme 4.2).

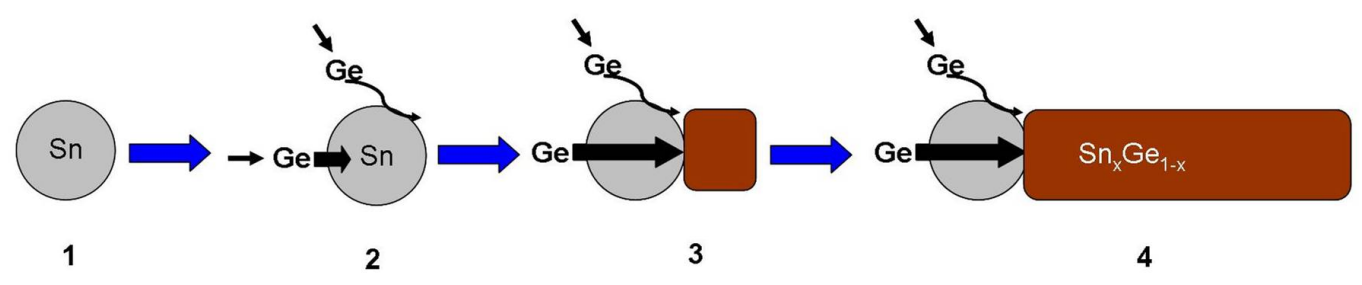

Scheme 4.2 Tin germanium nanowire growth mechanism.

In the first stage, the $\mathrm{Sn}$ precursor was reduced by $\mathrm{NaBH}_{4}$ at $300{ }^{\circ} \mathrm{C}$. The obtained Sn exists in the liquid phase as the reaction temperature $\left(300^{\circ} \mathrm{C}\right)$ is about 
$100{ }^{\circ} \mathrm{C}$ higher than the melting point of bulk $\mathrm{Sn}\left(232^{\circ} \mathrm{C}\right)$. Because of surface tension, the free surface energy of a liquid Sn tends to minimize surface area. Hence, the Sn droplets take a roughly spherical shape.

In the second stage, the Ge precursor is reduced by $\mathrm{NaBH}_{4}$ at $300{ }^{\circ} \mathrm{C}$. When $\mathrm{Ge}^{0}$ formed, they are attached on the surface of Sn droplet where they could either diffuse along the outer surface of the Sn droplet or penetrate it. The solubility of Ge in liquid Sn (about 1.07 atomic $\%$ at $300{ }^{\circ} \mathrm{C}$ ) is slightly higher than the solubility of Ge in solid Sn (0.6 atomic\% $),{ }^{18}$ but it is still a limited solubility.

In the third stage, nucleation formed and the nanowire starts to grow. The nanowire growth consumes $\mathrm{Ge}^{0}$ leading to a lower concentration of $\mathrm{Ge}^{0}$ at the growing zone than the rest of the surface. A concentration gradient of $\mathrm{Ge}^{0}$ exists along the surface driving further growth (stage 4).

In the fourth stage, the gradient of $\mathrm{Ge}^{0}$ concentration leads to a growth rate and forms a long nanowire.

\subsection{Summary}

In conclusion, the results presented above have demonstrated that high yield, high aspect ratio and straight tin germanium alloy nanowires can be successfully synthesized through a simple one pot solution phase synthesis, using Sn nanoparticle as a self-catalyst, via SLS growth mechanism. This is the first reported synthesis of a one-dimensional nanostructure tin germanium alloy. The reaction temperature, size of Sn nanoparticles, Sn/Ge precursors ratio and injection rate of Ge precursor significantly influence the morphology, length and diameter of as-prepared tin germanium nanowires. Moreover, it is worth not- 
ing that $\alpha$-Sn with trace amounts of Ge impurity was present in the nanoparticle product obtained at $200{ }^{\circ} \mathrm{C}$. This result indicates that $\alpha$-Sn can be stable at room temperature which has not been reported in the literature.

XRD and EDX/SEM measurements indicate that the $\mathrm{Sn}$ concentration in $\mathrm{Sn}_{x} \mathrm{Ge}_{1-x}$ nanowires obtained at an optimal reaction condition is $0.1\left(\mathrm{Sn}_{0.1} \mathrm{Ge}_{0.9}\right)$. This ratio is equal to the critical ratio reported in literature. For bulk $\mathrm{Sn}_{x} \mathrm{Ge}_{1-x}$ was considered to have a direct band gap when $x>0.1$ and an indirect band gap when $x<0.1 .^{21}$

Furthermore, EDX/STEM characterization revealed that significant concentration changes of both Ge and Sn occur in the growing zone, from a Sn-rich seed to a Ge-rich nanowire. This can account for the observed lattice distortion in the growing zone.

The as-prepared nanowires with face-centered cubic structure are primarily oriented in the $<110>$ direction, some in the $<112>$ direction, and a few in $<111>$ directions. Moreover, HRTEM characterization demonstrated that the formation of $\{111\}<112>$ twinning begins from the nucleation stage of nanowire growth. Highly curved interface favors growth of $<112>$ twins while nearly flat interface tends to grow $<110>$ oriented single crystal nanowires.

In future work, characterization of the optical and electrical properties of asprepared tin germanium nanowires is to be completed. Since the tapered end of the nanowire may affect the future application in optoelectronic devices, the synthetic approach can be further modified by varying the reaction parameter like surfactants and Ge precursors. 


\section{REFERENCES}

[1] Wang, D.; Dai, H. Applied Physics A 2006, 85, 217-225.

[2] Kodambaka, S.; Tersoff, J.; Reuter, M.; Ross, F. Science 2007, 316, 729-732.

[3] Ge, M.; Liu, J.; Wu, H.; Yao, C.; Zeng, Y.; Fu, Z.; Zhang, S.; Jiang, J. The Journal of Physical Chemistry C 2007, 111, 11157-11160.

[4] Tuan, H.-Y.; Lee, D. C.; Hanrath, T.; korgel, B. A. Chemistry of Materials $2005,17,5705-5711$.

[5] Schricker, A. D.; Joshi, S. V.; Hanrath, T.; Banerjee, S. K.; Korgel, B. A. Journal of Physical Chemistry B 2006, 110, 6816-6823.

[6] Bruno, M.; Palummo, M.; Ossicini, S.; Del Sole, R. Surface Science 2007, 601, 2707-2711.

[7] Chan, C. K.; Patel, R. N.; O Connell, M. J.; Korgel, B. A.; Cui, Y. ACS Nano 2010, 4, 1443-1450.

[8] Sivakov, V.; Andra, G.; Gawlik, A.; Berger, A.; Plentz, J.; Falk, F.; Christiansen, S. H. Nano Letters 2009, 9, 1549-1554.

[9] Liang, L.; Xiaosheng, F.; Han Guan, C.; Fei, Z.; Tze Haw, L.; Xijin, X.; Yunxia, Z.; Shusheng, P.; Guanghai, L.; Lide, Z. Advanced Functional Materials 2008, 18, 1080-1088.

[10] Zhao, Y.; Zhang, Z.; Dang, H. Materials Science and Engineering A 2003, 359, 405-407.

[11] Kumar, C. Nanomaterials for Medical Diagnosis and Therapy; Wiley-Vch Verlag Gmbh \& Co.KGaA, Weinheim, 2007; Vol. 10.

[12] Paul.W, Semiconductor Science and Technology 1990, s1-s4. 
[13] Jenkins, D. W.; Dow, J. D. Phys. Rev. B 1987, 36, 7994-8000.

[14] Guevara, L. d.; Perez, H.; Rodriguez, A. G.; Navarro-Contreras, H.; Vidal, M. A. Applied Physics Letters 2004, 84, 4532-4534.

[15] He, G.; Atwater, H. A. Phys. Rev. Lett. 1997, 79, 1937-1940.

[16] Lyman, P. F.; Bedzyk, M. J. Applied Physics Letters 1996, 69, 978 - 980.

[17] Nakayama, Y.; Takase, K.; Hirahara, T.; Hasegawa, S.; Okuda, T.; Harasawa, A.; Matsuda, I.; Nakamura, Y.; Ichikawa, M. Japanese Journal of Applied Physics 2007, 46, 1176-1178.

[18] Hansen, M.; Elliot, R. P. Constitution of binary alloys First supplement; McGraw Hill, 1965.

[19] Taraci, J.; Zollner, S.; McCartney, M. R.; Menendez, J.; SantanaAranda, M. A.; Smith, D. J.; Haaland, A.; Tutukin, A. V.; Gundersen, G.; Wolf, G.; Kouvetakis, J. Journal of the American Chemical Society 2001, 123, 10980-10987.

[20] Lide, D. R. CRC handbook of chemistry and physics, 88th ed.; Taylor \& Francis Group LLC, 2008.

[21] Guevara, L. d.; Perez, H.; Rodriguez, A.; Navarro-Contreras, H.; Vidal, M. A. Applied Physics Letters 2003, 83, 4942 - 4944.

[22] Chizmeshya, A. V. G.; Bauer, M. R.; Kouvetakis, J. Chemistry of Materials 2003, 15, 2511-2519.

[23] Mathur, S.; Shen, H.; Sivakov, V.; Werner, U. Chemistry of Materials 2004, $16,2449-2456$.

[24] Kuykendall, T.; Pauzauskie, P.; Lee, S.; Zhang, Y.; Goldberger, J.; Yang, P. Nano Letters 2003, 3, 1063-1066. 
[25] Wu, Y.; Yang, P. Journal of the American Chemical Society 2001, 123, 31653166.

[26] Hanrath, T.; Korgel, B. A. Advanced Materials 2003, 15, 437-440.

[27] Wang, F.; Dong, A.; Sun, J.; Tang, R.; Yu, H.; Buhro, W. E. Inorganic Chemistry 2006, 45, 7511-7521.

[28] Heitsch, A. T.; Fanfair, D. D.; Tuan, H.-Y.; Korgel, B. A. Journal of the American Chemical Society 2008, 130, 5436-5437.

[29] Lu, X.; Fanfair, D. D.; Johnston, K. P.; Korgel, B. A. Journal of the American Chemical Society 2005, 127, 15718-15719.

[30] Hanrath, T.; Korgel, B. A. Journal of the American Chemical Society 2002, 124, 1424-1429.

[31] Hanrath, T.; Korgel, B. A. Journal of the American Chemical Society 2004, 126, 15466-15472.

[32] Henderson, E. J.; Hessel, C. M.; Veinot, J. G. C. Journal of the American Chemical Society 2008, 130, 3624-3632.

[33] Joo, Y. J.; Takemoto, T. Materials Letters 2002, 56, 793-796.

[34] Sun, Y.; Yin, Y.; Mayers, B. T.; Herricks, T.; Xia, Y. Chemistry of Materials 2002, 14, 4736-4745.

[35] Gu, G.; Burghard, M.; kIM, G.; Dusberg, G.; Chiu, P.; Krstic, V. Journal of Applied Physics 2001, 90, 5747-5751.

[36] Hanrath, T.; Korgel, B. A. Small 2005, 1, 717-721.

[37] Egerton, R. F. Physical Principles of Electron Microscopy: An Introduction to TEM, SEM, and AEM; Springer, 2005; pp 125-140. 
[38] Trentler, T. J.; Hickman, K. M.; Goel, S. C.; Viano, A. M.; Gibbons, P. C.; Buhro, W. E. Science 1995, 270, 1791-1794.

[39] Buhro, W. E.; Hickman, K. M.; Trentler, T. J. Advanced Materials 1996, 8, 685-688.

[40] Davidson, F. M.; Lee, D. C.; Fanfair, D. D.; Korgel, B. A. The Journal of Physical Chemistry C 2007, 111, 2929-2935.

[41] Wang, Z. W.; Li, Z. Y. Nano Letters 2009, 9, 1467-1471.

[42] Shimizu, T.; Xie, T.; Nishikawa, J.; Shingubara, S.; Senz, S.; Gosele, U. Advanced Materials 2007, 19, 917-928.

[43] Schmidt, V.; Senz, S.; Gosele, U. Nano Letters 2005, 5, 931-935.

[44] Johansson, J.; Karlsson, L. S.; Dick, K. A.; Bolinsson, J.; Wacaser, B. A.; Deppert, K.; Samuelson, L. Crystal Growth $\mathcal{E}$ Design 2008, 9, 766-773.

[45] Hidalgo, P.; Mendez, B.; Piqueras, J. Nanotechnology 2008, 19, 455705.

[46] Cheyssac, P.; Sacilotti, M.; Patriarche, G. Journal of Applied Physics 2006, $100,044315$.

[47] Rogers, R. R.; Fydell, J. F. Journal of The Electrochemical Society 1953, 100, 383-387.

[48] Wagner, R. S.; Ellis, W. C. Applied Physics Letters 1964, 4, 89. 



\section{Chapter 5}

\section{Synthesis of Tin Monosulfide Nanoparticles}

\section{Contents}

5.1 Introduction . . . . . . . . . . . . . . . . . 129

5.2 One pot synthesis of water-soluble SnS nanoparticles . . . 133

5.3 Summary . . . . . . . . . . . . . . . 155

\subsection{Introduction}

This chapter is concerned with the development of a novel one-pot synthesis of water-soluble SnS nanoparticles. The synthetic approach described here involves the reaction of inorganic starting materials $\mathrm{SnBr}_{2}$ and $\mathrm{Na}_{2} \mathrm{~S}$ in the presence of various ethanolamine derivatives in ethylene glycol.

Research on the synthesis of narrow band gap IV-VI semiconductor nanoparticles, such as PbS, SnS, and SnTe has attracted increasing interest as they are optically active in the near infrared region (NIR) and infrared region (IR), thus 
they have potential applications in photovoltaics, near-infrared detectors and biomedical applications such as targeted cancer cell hyperthermia. ${ }^{1-4}$ Current research on the synthesis of SnS nanoparticles was motivated by this potential. The development of the synthesis of water-soluble SnTe will be reported in the Chapter 6.

In the past decade, much attention has been given to the synthesis of monodispersed chalcogenide nanoparticles with cubic (isotropic unit cell) crystal structures such as CdS, CdSeS, CdSe and PbS due to their ease of synthesis. ${ }^{5-9}$ In contrast, SnS adopts an orthorhombic (anisotropic unit cell) layered crystal structure where the $\mathrm{Sn}$ and S atoms are tightly bonded in the layer and the layers are bonded by weak van der Waals forces. ${ }^{1,10}$ The synthesis of uniform size IV-VI semiconductor nanoparticles with layered structures that are small enough to be in the quantum confinement regime is still a significant challenge.

Conventional SnS synthetic techniques such as spray pyrolysis, ${ }^{11}$ chemical vapour deposition ${ }^{12}$ and electron beam evaporation ${ }^{13}$ have been applied most often for the fabrication of bulk SnS films. Investigations into SnS nanoparticles synthesis are comparatively few. As shown in Table 5.1, Gou et al. ${ }^{14}$ reported that SnS nanoparticles can be prepared through a precipitation reaction between $\mathrm{SnCl}_{2} \cdot 2 \mathrm{H}_{2} \mathrm{O}$ and thioacetamide (TAA) in an acidic aqueous solution at $60{ }^{\circ} \mathrm{C}$ for $1.5 \mathrm{~h}$. The nanoparticles are in the range of $30-100$ $\mathrm{nm}$. Schlecht and coworkers ${ }^{15}$ prepared SnS nanoparticles through a mild solvothermal route, where an activated metallic tin and elemental sulphur were mixed in diethyleneglycoldimethylether (diglyme) at $160{ }^{\circ} \mathrm{C}$ for $24 \mathrm{~h}$. The particles size are in the range of $20-40 \mathrm{~nm}$. Koktysh et al. ${ }^{16}$ presented a synthesis of SnS nanoparticles by a solvothermal decomposition of a singlesource tin sulphide precursor, bis(diethyldithiocarbamato)tin(II) $\left(\mathrm{Sn}\left(\mathrm{Et}_{2} \mathrm{Dtc}\right)_{2}\right)$, 
in oleylamine(OLA)/oleic acid (OA) mixture at $170{ }^{\circ} \mathrm{C}$ for $30 \mathrm{~s}$. The prepared nanoparticles are relatively small but the size distribution is broad with a range of 10 to $20 \mathrm{~nm}$. To the best of our knowledge, there was only one report by Hickey et al. ${ }^{17}$ demonstrating a method of forming SnS nanoparticles of less than $10 \mathrm{~nm}$ in size and none with size less than $7 \mathrm{~nm}$. The synthesis of Hickey and coworkers uses the air sensitive organometallic complex $\operatorname{Sn}\left[\mathrm{N}\left(\mathrm{SiMe}_{3}\right)_{2}\right]_{2}$ as a tin precursor and TAA as the sulphur precursor. Trioctylphosphine (TOP) and OA were used as surfactant in the reaction which render the synthesized SnS nanoparticles hydrophobic. More recently, Liu et al. ${ }^{18}$ reported the synthesis of around $6 \mathrm{~nm} \mathrm{SnS} \mathrm{nanoparticles} \mathrm{from} \mathrm{SnCl}_{2}$ and bis(trimethylsily)sulphide (TMS) in OLA at $120^{\circ} \mathrm{C}$ for $5 \mathrm{~min}$. However, the direct product is still soluble only in organic solvents, which to some extent limits their use in the biomedical field. ${ }^{19}$

Table 5.1 A summary of the synthesis of SnS nanoparticles in literature.

\begin{tabular}{|l|l|l|l|l|l|l|}
\hline Author & Precursors & Surfactant & Solvent & $\begin{array}{l}\text { Temp } \\
\left({ }^{\circ} \mathrm{C}\right)\end{array}$ & Time & $\begin{array}{l}\text { NPs } \\
(\mathrm{nm})\end{array}$ \\
\hline Gou et al. ${ }^{14}$ & $\begin{array}{l}\mathrm{SnCl}_{2} \cdot 2 \mathrm{H}_{2} \mathrm{O} \\
\mathrm{TAA}\end{array}$ & - & $\mathrm{H}_{2} \mathrm{O}$ & 60 & $1.5 \mathrm{~h}$ & $30-100$ \\
\hline Schlecht et al. ${ }^{15}$ & $\begin{array}{l}\text { metallicSn } \\
\text { Elemental S }\end{array}$ & - & diglyme & 160 & $24 \mathrm{~h}$ & $20-40$ \\
\hline Koktysh et al. ${ }^{16}$ & $\mathrm{Sn}\left(\mathrm{Et}_{2} \text { Dtc }\right)_{2}$ & $\begin{array}{l}\text { OLA } \\
\text { OA }\end{array}$ & OLA & 170 & $30 \mathrm{~s}$ & $10-20$ \\
\hline Hickey et al. ${ }^{17}$ & ${\mathrm{Sn}\left[\mathrm{N}\left(\mathrm{SiMe}_{3}\right)_{2}\right]_{2}}_{2}$ & $\begin{array}{l}\text { TOP } \\
\text { OA }\end{array}$ & ODE & 170 & $3 \mathrm{~min}$ & $\sim 7$ \\
\hline Liu et al. ${ }^{18}$ & $\begin{array}{l}\mathrm{SnCl}_{2} \\
\mathrm{TMS}\end{array}$ & $\begin{array}{l}\text { OLA } \\
\text { OA }\end{array}$ & ODE & 120 & $5 \mathrm{~min}$ & $\sim 6$ \\
\hline
\end{tabular}

Since the pioneering work of Chan and $\mathrm{Nie}^{20}$ and Alivisatos et al., ${ }^{21}$ where nanoparticles were made water soluble through surface modification, various methods have been developed, in which hydrophobic nanoparticles are first prepared and then the hydrophobic surface ligands are replaced by watersoluble biofunctional molecules, such as mercaptoacetic acid (MPA), ${ }^{20,22,23}$ thio- 
glycolic acid (TGA) ${ }^{24}$ and dithiothreitol (DTT) ${ }^{25}$ Common to these molecules, one end is tethered to the nanoparticle surface (for example -SH group in MPA) and the other end (-COOH group for MPA) is hydrophilic(Scheme 5.1).

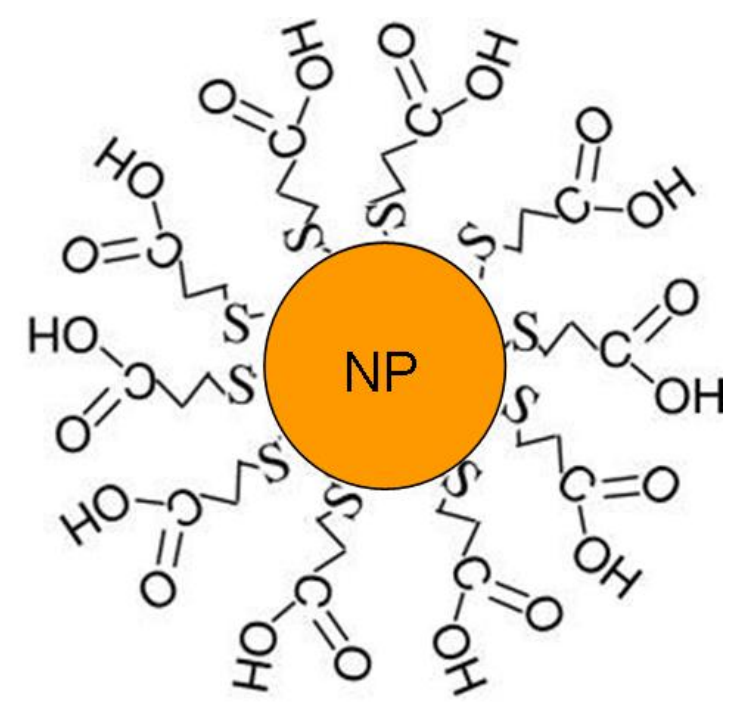

Scheme 5.1 Schematic representation of a water-soluble nanoparticle stabilized by MPA ligands.

However, these ligand exchange process are usually time-consuming, from several hours to a couple of days. ${ }^{26}$ Hence, the direct synthesis of water-soluble SnS nanoparticle is desirable.

To achieve one-pot synthesis of water-soluble nanoparticles it is required to perform reactions either in an aqueous solution ${ }^{27}$ or in a hydrophilic organic solution. ${ }^{28} \mathrm{SnS}$ has previously been synthesized in $\mathrm{H}_{2} \mathrm{O}$ using bifunctional surfactant TGA, together with $\mathrm{SnCl}_{2} \cdot 4 \mathrm{H}_{2} \mathrm{O}$ and $\mathrm{Na}_{2} \mathrm{~S}$ or $\mathrm{NH}_{2} \mathrm{CSNH}_{2}$ or $\mathrm{CH}_{3} \mathrm{CSNH}_{2}$ as the tin and sulfur precursor, respectively, but it produced nanorods or nanosheets rather than nanoparticles. ${ }^{29}$ Moreover, Peng and co-workers presented a synthesis of unprotected $\mathrm{SnS}$ in ethylene glycol through $\mathrm{SnCl}_{2} \cdot 2 \mathrm{H}_{2} \mathrm{O}$ and $\mathrm{Na}_{2} \mathrm{~S} .{ }^{30}$ The resulting products were clusters without any size control. However the potential future applications of the particles were significantly affected by the effect of the size distribution on the size dependent physical properties. ${ }^{31}$ 
In solution phase synthesis, the particle size and shape can be tailored by modifying the experimental conditions such as reaction temperature, precursors and surfactant. ${ }^{32}$ Typically, surfactants play a crucial role during the formation of nanoparticles. Hence, optimization of the surfactant in the reaction system is expected to yield size controlled nanoparticles.

The objectives of this research were to develop a facile, one-pot and inexpensive synthetic route to produce (1) monodispersed SnS nanoparticles of a few nanometers in size; with (2) the nanoparticles having a hydrophilic surface coating.

\subsection{One pot synthesis of water-soluble SnS nanopar- ticles}

Surfactants play a crucial role in yielding size controlled nanoparticles. In the current research, various ethanolamine derivatives such as $\mathrm{N}, \mathrm{N}$-dimethylethanolamine (DMEA), N-methyldiethanolamine (MDEA) and triethanolamine (TEA) as shown in Scheme 5.2 were selected and used as surfactants, which have not been used in any previous SnS nanoparticles synthesis. Ethanolamines are versatile, polyfunctional molecules that combine the characteristics of amines and alcohols. The use of ethanolamines has five benefits: (1) they have an -OH group which is hydrophilic; ${ }^{33}$ (2) they may form chelate complexes with $\mathrm{Sn}^{2+}$ ions; ${ }^{34}$ (3) they are easy to attach to the surface of the nanoparticles and stabilize them through lone pair electrons from $\mathrm{N}$ and/or $\mathrm{O}$ atoms;(4) they have mild basicity which plays an important role in the formation of SnS nanoparticles as it has been reported in literature; ${ }^{35}$ and (5) they are inexpensive chemicals. 


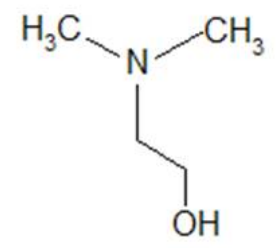

DMEA

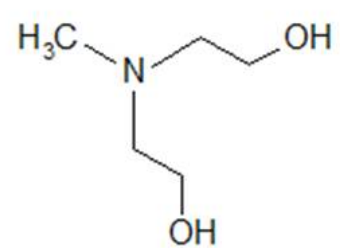

MDEA

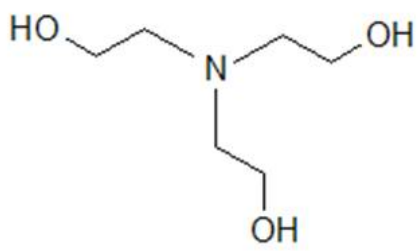

TEA

Scheme 5.2 The chemical structure of DMEA, MDEA and TEA.

Readily available inorganic starting materials, $\mathrm{SnBr}_{2}$ and $\mathrm{Na}_{2} \mathrm{~S}$ were used as they are relatively cheaper and more reactive. From Sigma-Aldrich website (2010), $10 \mathrm{~g} \mathrm{SnBr}_{2}$ costs only about $\mathrm{NZ} \$ 54$, which is cheaper than most of other tin sources such as $\mathrm{SnCl}_{2}(10 \mathrm{~g}, \mathrm{NZ} \$ 252)$ and $\mathrm{Sn}\left[\mathrm{N}\left(\mathrm{SiMe}_{3}\right)_{2}\right]_{2}(5 \mathrm{~mL}, \mathrm{NZ} \$$ 82). To our knowledge, the use of $\mathrm{SnBr}_{2}$ as a tin source has not been reported in previous $\mathrm{SnS}$ syntheses. Moreover, it is also known that $\mathrm{Na}_{2} \mathrm{~S}$ is more reactive than most of other conventionally used sulphur sources such as $\mathrm{NH}_{2} \mathrm{CSNH}_{2}$ and $\mathrm{CH}_{3} \mathrm{CSNH}_{2} \cdot{ }^{29} \mathrm{Na}_{2} \mathrm{~S}$ can easily release $\mathrm{S}^{2-}$ at room temperature while the decomposition of $\mathrm{NH}_{2} \mathrm{CSNH}_{2}$ or $\mathrm{CH}_{3} \mathrm{CSNH}_{2}$ occurs at higher temperatures, such as $80{ }^{\circ} \mathrm{C}$ for $\mathrm{NH}_{2} \mathrm{CSNH}_{2} \cdot{ }^{36}$ Moreover, $\mathrm{Na}_{2} \mathrm{~S}$ is very cheap as $100 \mathrm{~g} \mathrm{Na}_{2} \mathrm{~S}$ $(98 \%)$ is only $\mathrm{NZ} \$ 50$.

The use of ethylene glycol as the hydrophilic organic solvent has four benefits: (1) it readily dissolves ethanolamines and $\mathrm{SnBr}_{2}$ forming clear colorless solution; (2) in ethylene glycol, tin halide do not produce hydrolysis product such as $\mathrm{Sn}(\mathrm{OH})_{2}$ which is formed in aqueous solutions; ${ }^{37}$ (3) it enables the tuning of the reaction temperature over a range from $0{ }^{\circ} \mathrm{C}$ to $200{ }^{\circ} \mathrm{C}$, broader than some other commonly used hydrophilic organic solvents, such as tetrahydrofuran (THF, BP: $\left.66^{\circ} \mathrm{C}\right)$; and (4) it is relatively cheap (1 L, NZ $\$ 135$, Sigma-Aldrich). 


\subsubsection{Experiment A-C : Effect of Surfactant}

In this section, all reactions were performed at room temperature in ethylene glycol with different ethanolamine derivatives, using $\mathrm{SnBr}_{2}$ and $\mathrm{Na}_{2} \mathrm{~S}$ as the tin and sulfur sources, respectively.

\section{Experimental}

In a $50 \mathrm{~mL}$ three necked round bottomed flask, $0.056 \mathrm{~g}(0.2 \mathrm{mmol}) \mathrm{SnBr}_{2}$ was dissolved in a mixture of $20 \mathrm{~mL}$ ethylene glycol and $4 \mathrm{~mL}$ ethanolamine derivative with vigorous stirring. $2 \mathrm{~mL}$ of $0.1 \mathrm{M}$ solution of $\mathrm{Na}_{2} \mathrm{~S}$ in ethylene glycol was added dropwise over 10 min into the vigorously stirred $\mathrm{SnBr}_{2}$ solution. The resulting brown-coloured SnS nanoparticles were separated out via centrifugation (50,000 rpm for 1-2 h) and washed repeatedly in ethanol four times and stored in a desired solvent for subsequent morphology and optical properties measurement. All the syntheses were performed under Argon.

\section{Results and discussion}

Fig. 5.1a shows a typical TEM image of the products obtained using DMEA, a tertiary aliphatic amine with a single hydroxyl group, as surfactant. The nanoparticles are spherical or oval in shape. A histogram showing the nanoparticles size distribution taken from over 100 nanoparticles is shown in Fig. 5.1b and indicates an average size of $5.0 \pm 4.0 \mathrm{~nm}$. More than $12 \%$ of nanoparticles are larger than $6.5 \mathrm{~nm}$.

When MDEA, a tertiary amine with two hydroxyl groups, was used, the resulting SnS nanoparticles were roughly spherical in shape (Fig. 5.1c) with an average size of $4.0 \pm 2.0 \mathrm{~nm}$ (Fig. $5.1 \mathrm{~d}$ ). 


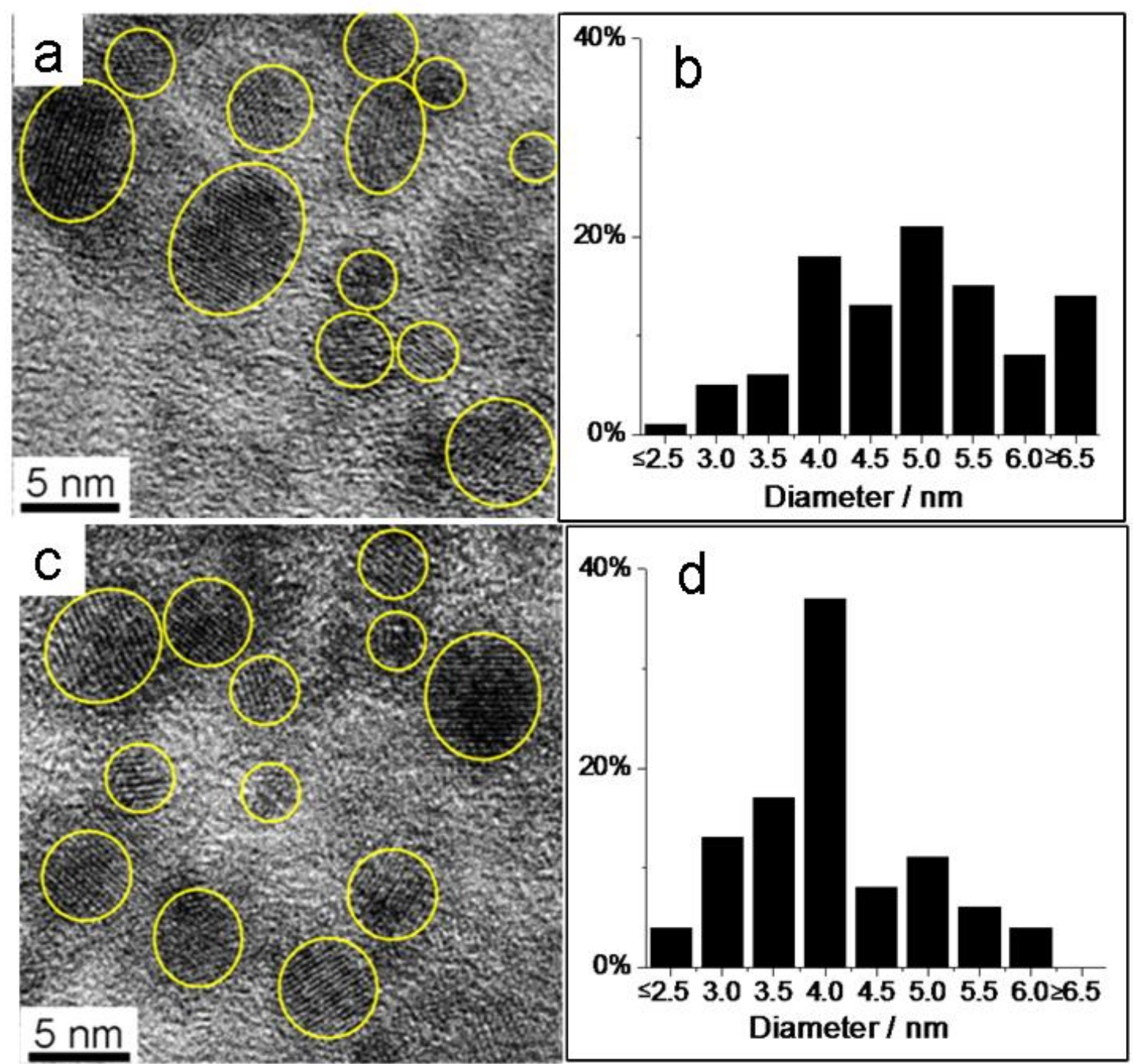

Fig. 5.1 HRTEM images of SnS nanoparticles synthesized in DMEA (a) and MDEA (c) at room temperature, respectively. The corresponding histogram of size distributions for the SnS nanoparticles showing in (b) and (d)

Fig. 5.2 shows typical TEM images of the reaction product synthesized using TEA, a tertiary amine with three hydroxyl groups. The nanoparticles are approximately spherical in shape. The inset, Fig. 5.2b, shows an HRTEM image of an individual nanocrystal with the atomic lattice fringes clearly visible, demonstrating the highly crystalline nature of the SnS nanoparticles. A histogram, as shown in Fig. 5.2c, indicates an average nanoparticle size of $3.2 \pm$ $0.5 \mathrm{~nm}$. 


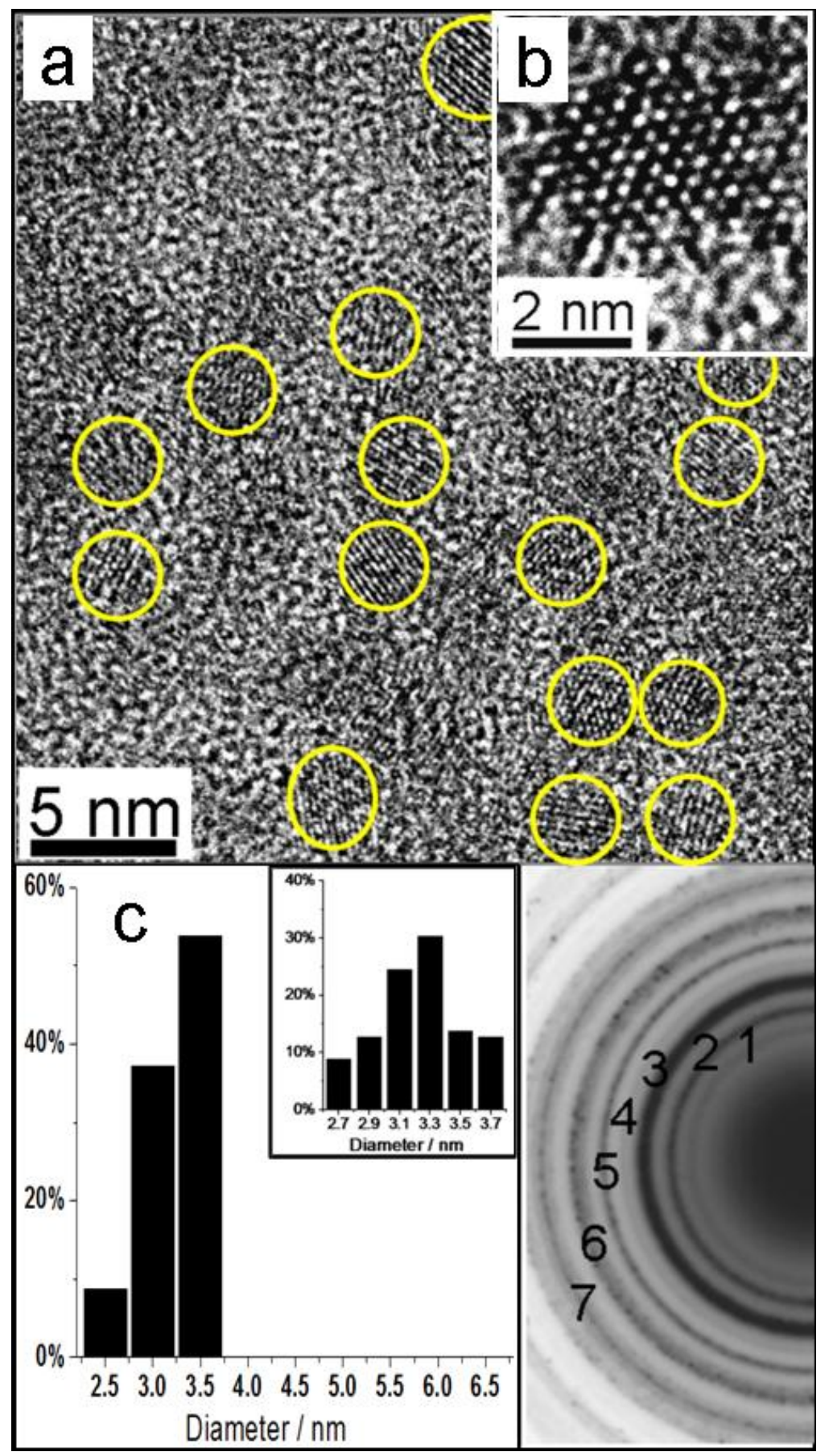

Fig. 5.2 (a) and (b) TEM images showing crystalline SnS nanoparticles obtained with TEA, (c) histogram of nanoparticle size distribution, (d) selected area electron diffraction (SAED) pattern of orthorhombic SnS nanoparticles.

Directly comparing our results to those of Peng et al. ${ }^{30}$ is complicated by the use of different tin precursors (Peng: $\mathrm{SnCl}_{2}$; this work: $\mathrm{SnBr}_{2}$ ) however the effect of this is assumed to be negligible, and the use of the stabilizer ethanolamine is crucial for the successful synthesis of SnS nanoparticles. Moreover, the 
results also indicate that $\mathrm{SnS}$ nanoparticle size and monodispersity can be controlled by the number of hydroxyl groups on the stabilizer. As summarized in Table 5.2, TEA (three hydroxyl groups) produced smaller and more monodisperse nanoparticles than MDEA (two hydroxyl groups) which in turn formed smaller and more monodisperse nanoparticles than DMEA (one hydroxyl group). The greater monodispersity with the increasing number of hydroxyl groups most likely arises from the ability of multiple hydroxyl groups to bind more strongly to the nanocrystals as they grow.

Table 5.2 Summary of results for SnS nanoparticles obtained with various ethanolamine derivatives.

\begin{tabular}{|c|c|c|c|c|c|}
\hline Exp & Ethanolamine & Volume $(\mathrm{mL})$ & Temperature & Injection & products \\
\hline A & DMEA & 4 & RT & dropwise & $5.0 \pm 4.0 \mathrm{~nm}$ \\
\hline B & MDEA & 4 & RT & dropwise & $4.0 \pm 2.0 \mathrm{~nm}$ \\
\hline C & TEA & 4 & RT & dropwise & $3.2 \pm 0.5 \mathrm{~nm}$. \\
\hline
\end{tabular}

Previous reports show that TEA complexes with $\mathrm{Sn}^{2+}$ to form a chelate compound $[\mathrm{Sn}(\mathrm{TEA}) \mathrm{n}]^{2+} .{ }^{34,38-40}$ A proposed reaction for the formation of SnS is illustrated in Scheme 5.3 proceeding via an $[\mathrm{Sn}(\mathrm{TEA}) \mathrm{n}]^{2+}$ intermediate. This formation mechanism is different to the Peng's approach in which $\mathrm{SnCl}_{2}$ directly reacts with $\mathrm{Na}_{2} \mathrm{~S}$ forming SnS. ${ }^{30}$ DMEA and MDEA also form complexes with tin that are less stable than the TEA complex.

\section{$\mathrm{Sn}^{2+}+\mathrm{nTEA} \rightleftharpoons\left[\mathrm{Sn}(\mathrm{TEA})_{\mathrm{n}}\right]^{2+}$ \\ $\left[\mathrm{Sn}(\mathrm{TEA})_{\mathrm{n}}\right]^{2+}+\mathrm{S}^{2-} \longrightarrow \mathrm{SnS}+\mathrm{nTEA}$}

Scheme 5.3 Schematic illustration of the formation of SnS nanoparticles.

The selective-area electron diffraction (SAED) pattern taken from the product prepared with $4 \mathrm{~mL}$ TEA, Fig. 5.2d, has a sharp set of diffraction rings. 
These diffraction rings can be indexed to the planes of orthorhombic phase SnS (Pbnm, $a=11.143 \AA, b=3.971 \AA$ and $c=4.336 \AA)$ 1-(101); 2-(201), (210); 3(011), (111), (301), (400); 4-(211), (401); 5-(311), (410);6-(102), (020), (501), (112); and 7-(121), (511)). No cubic phase SnS was detected, which was further confirmed by powder X-ray diffraction (Fig. 5.3). Same results were also obtained from the samples produced using DMEA or MDEA as stabilizing ligands.

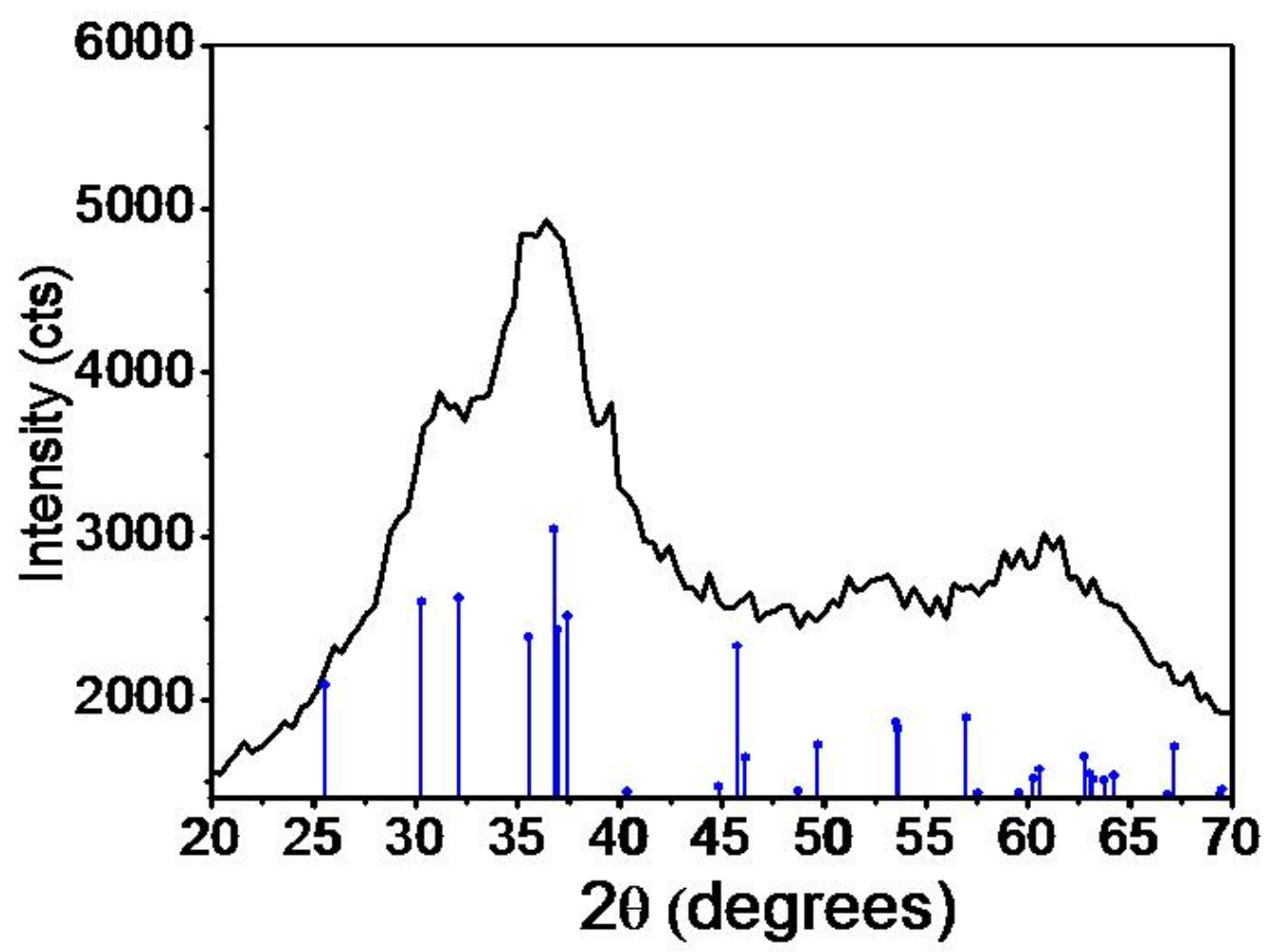

Fig. 5.3 XRD pattern of as prepared SnS nanoparticles (Co radiation). The expected positions and intensities for peaks corresponding to orthorhombic SnS (ICDD 01-071-3679) are marked by lines labeled with "•".

Energy-dispersive X-ray (EDX) analysis of the SnS nanoparticles chemical composition showed an atomic ratio of $S n$ to $S$ of $1.0: 1.0$, consistent with SnS (Fig. 5.4) There are two peaks at 2.0 Kev and 2.62 Kev (Fig. 5.4) which can not be identified by EDX database. 


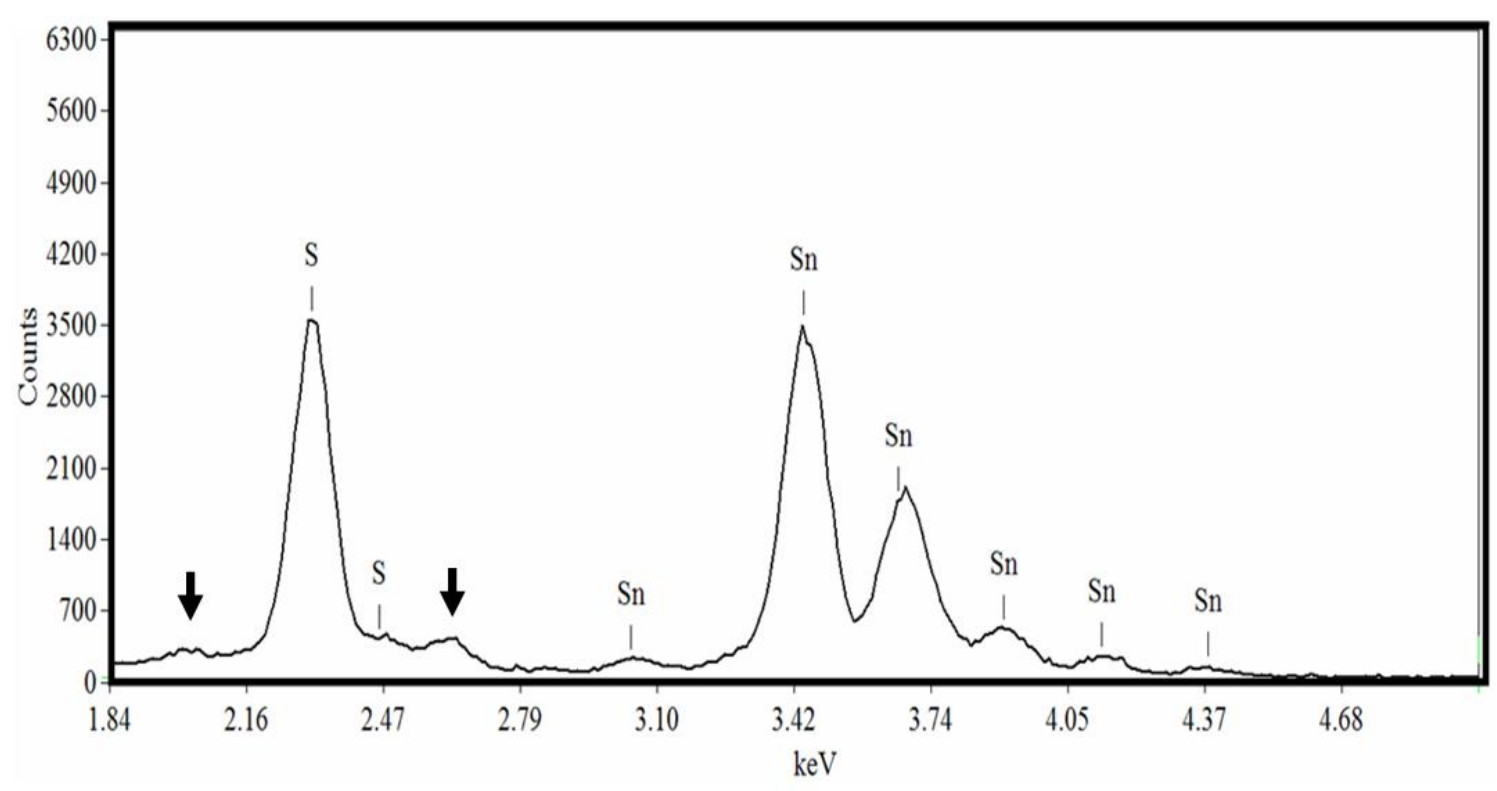

Fig. 5.4 Energy Dispersive X-ray (EDX) spectrum of SnS nanoparticles.The arrows denote unidentified peaks observed in this EDX spectrum only.

Reflectivity measurements were made upon drop-cast films of nanoparticles deposited on glass substrates. Fig. 5.5 shows the reflectivity data for films of the three nanoparticle samples. The smaller and more monodisperse samples produced using TEA and MDEA show a reflectivity edge that is blue-shifted by $\sim 0.4 \mathrm{eV}$ compared to the DMEA sample. The inset plot shows the first derivative of reflectivity with respect to incident photon energy, hv; peaks in these data correspond to the reflectivity edge of the semiconductor nanocrystals. The TEA and MDEA samples exhibit reflectivity edges of $\sim 1.65 \mathrm{eV}$. This value is significantly higher than the $1.1 \mathrm{eV}$ reported for the bulk band gap of SnS and comparable to $1.6 \mathrm{eV}$ reported for $\sim 7 \mathrm{~nm}$ SnS nanoparticles. ${ }^{11,16,17,41}$ In addition the more monodisperse TEA sample has a narrower first derivative peak than the MDEA sample. These observations can be explained by quantum effects arising from changes to the $\mathrm{SnS}$ band structure that occur in nanocrystals with a small diameter. In contrast the DMEA sample exhibits two peaks in the reflectance derivative at $\sim 1.6$ and $\sim 1.2 \mathrm{eV}$. This result is most likely due to two populations of nanoparticles in this sample, those with di- 
ameters above $6.5 \mathrm{~nm}$ that exhibit behavior similar to bulk SnS (peak at $\sim 1.2$ $\mathrm{eV}$ ) and those with diameters below $6.5 \mathrm{~nm}$ (peak at $\sim 1.6 \mathrm{eV}$ ).

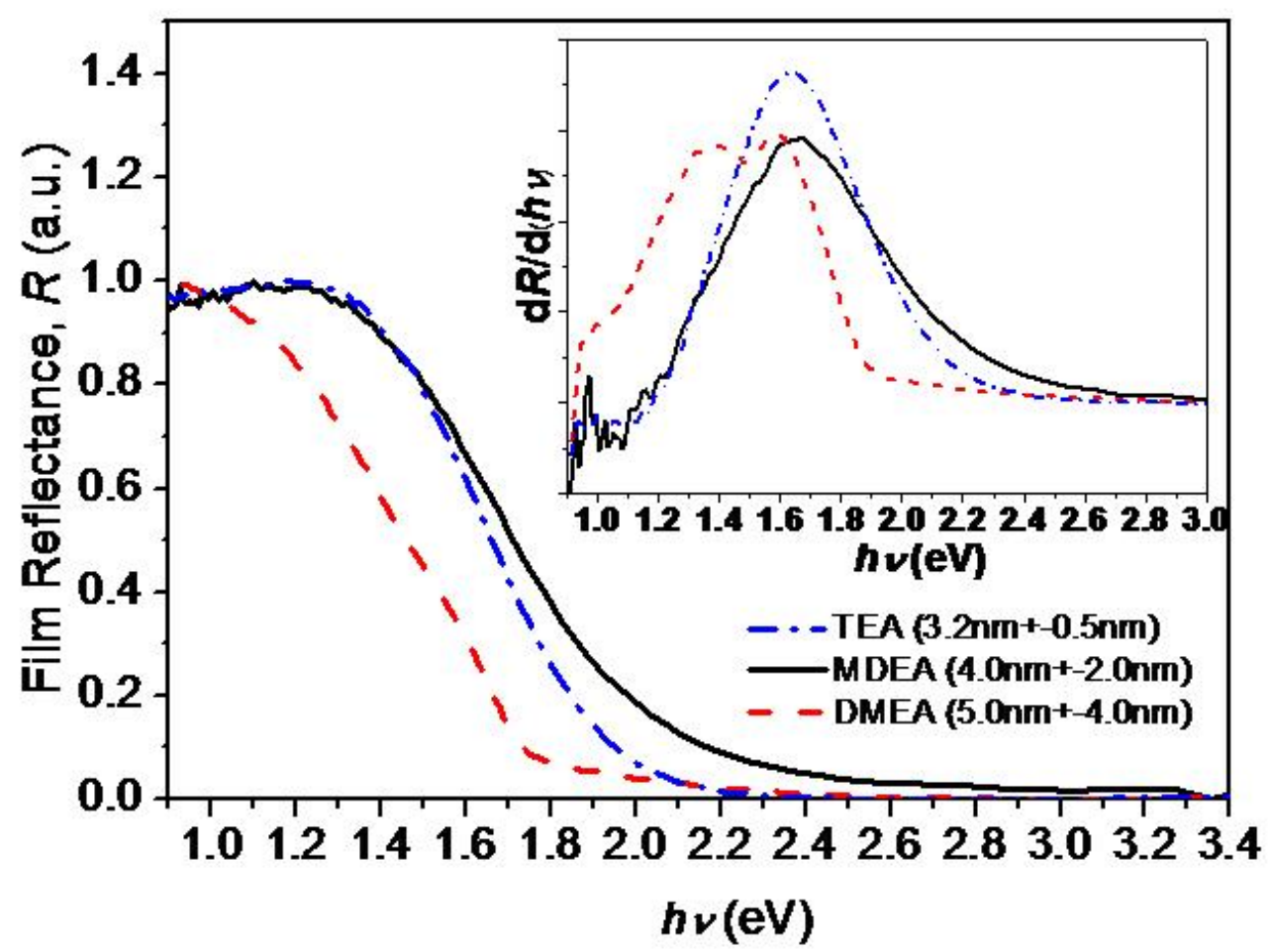

Fig. 5.5 Optical reflectance spectra of nanoparticle films. Inset: First derivative of film reflectance vs incident photon energy.

Fig. 5.6 shows UV-vis-NIR optical absorption data. Transmission measurements were performed upon colloidal nanoparticle dispersions in ethanol that were translucent brown in color (inset, Fig. 5.6). The solutions exhibit continuous absorption across the UV-vis spectrum, with increasing absorption near the UV region (inset, Fig. 5.6). For a sample behaving like a bulk indirect semiconductor, the absorption coefficient, $\alpha$, can be described by the relationship $(\alpha \mathrm{h} v)^{0.5} \propto$ photon energy, $\mathrm{h} v{ }^{42-44}$ This relationship is valid close to the indirect band gap edge, where the single parabolic band approximation holds. An estimate of the indirect band gap $\left(\mathrm{E}_{g}\right)$ of the sample can also be obtained from the intercept of this plot. ${ }^{42-44}$ Fig. 5.6 shows that the DMEA sample exhibits a 
region of linearity in $(\alpha \mathrm{h} v)^{0.5} \mathrm{vs} \mathrm{h} v$ and an estimated indirect band gap $\mathrm{E}_{g} \approx 1.1$ $\mathrm{eV}$, as indicated by extrapolation of the straight line. This reflects the absorption which is also shown in the inset of Fig. 5.5 for larger SnS particles with optical characteristics similar to those of the bulk material. Comparison with data from an amorphous SnS bulk film shows similar features to those of the sample obtained with the DMEA. ${ }^{11}$ There is no region of linearity observed for the smaller TEA and MDEA samples. This is as expected for quantum dots exhibiting a 0-D joint density of states and again illustrates that the nanocrystals are small enough to be in the quantum confinement regime. No fluorescence was observed from the sample which is consistent with previous research on SnS quantum dots. ${ }^{17}$ This could be due to fluorescence quenching that usually occurs in quantum dots without shell protection or the band structure.

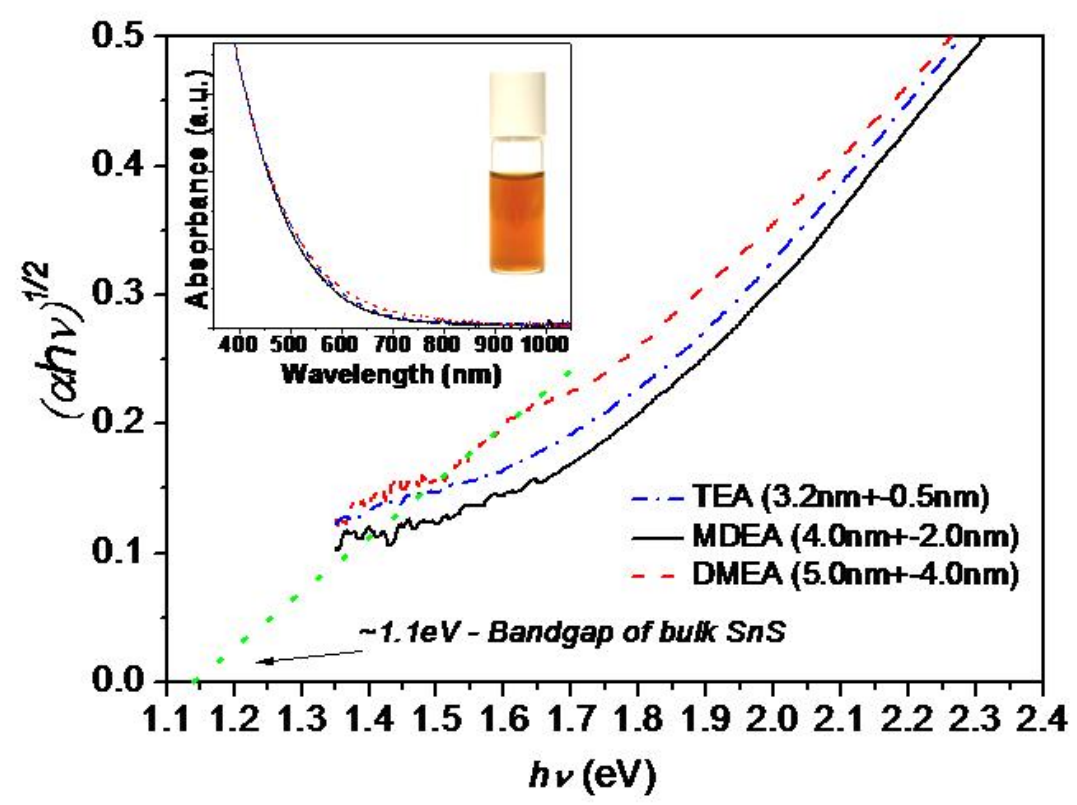

Fig. 5.6 The dependence of $(\alpha \mathrm{h} v)^{0.5}$ on photon energy $(\mathrm{h} v)^{45}$ and inset: Absorption spectra of SnS nanoparticles dispersed in ethanol (photograph) solution.

After purification, the surface bonding of SnS nanoparticles was characterized by FTIR spectroscopy and showed the presence of ethanolamine capping lig- 
ands (Fig. 5.7). The FTIR data obtained from different samples are summarized in Table 5.3.

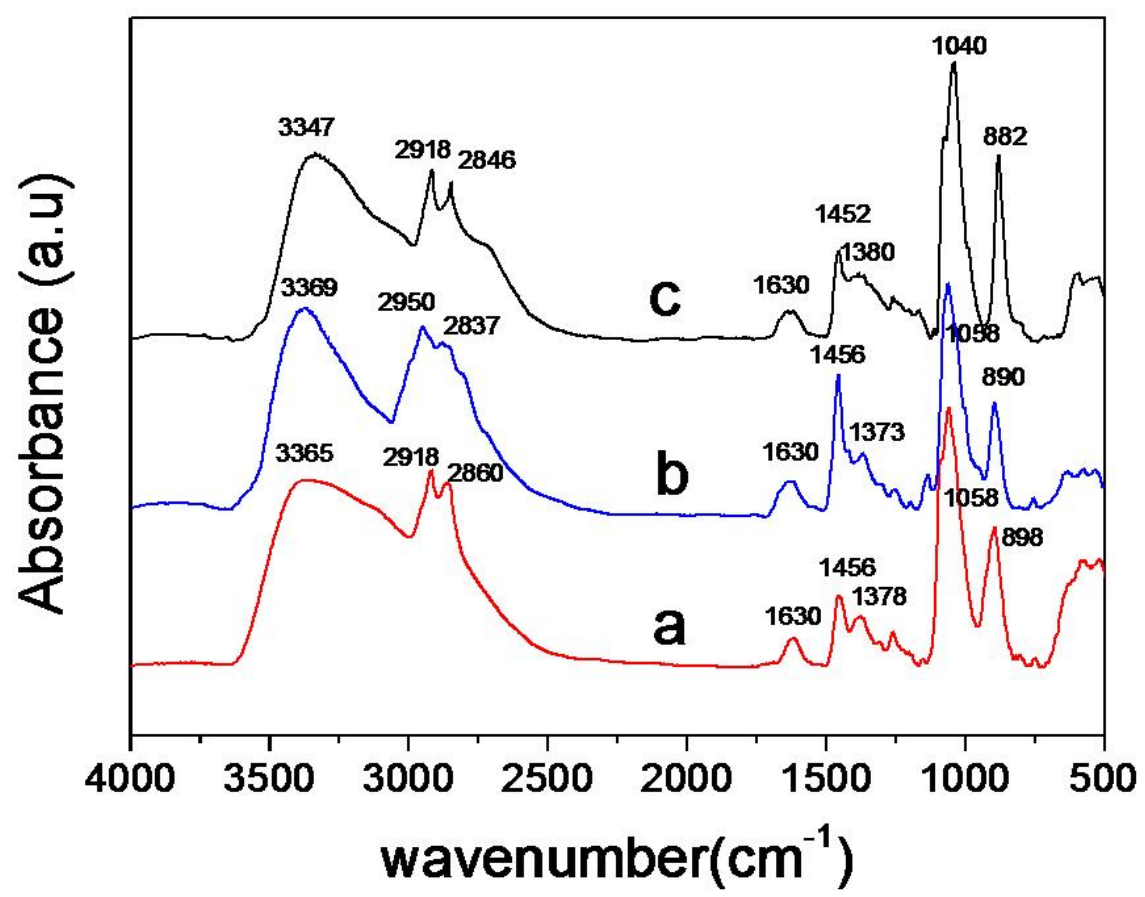

Fig. 5.7 FTIR spectra of (a) TEA-capped SnS nanoparticles, (b) MDEA-capped SnS nanoparticles, and (c) DMEA-capped SnS nanoparticles.

Table 5.3 Assignment of the FTIR Bands (in $\mathrm{cm}^{-1}$ ) of Fig. 5.7. ${ }^{10}$

\begin{tabular}{|l|l|l|l|}
\hline $\begin{array}{l}\text { TEA-capped } \\
\text { SnS NPs }\end{array}$ & $\begin{array}{l}\text { MDEA- } \\
\text { capped SnS } \\
\text { NPs }\end{array}$ & $\begin{array}{l}\text { DMEA- } \\
\text { capped SnS } \\
\text { NPs }\end{array}$ & Assignment \\
\hline 3365 & 3369 & 3347 & $\nu(\mathrm{O}-\mathrm{H})+\mathrm{O}-\mathrm{H}\left(\mathrm{H}_{2} \mathrm{O}\right)$ \\
\hline 2918,2860 & 2950,2837 & 2918,2846 & $\nu(\mathrm{C}-\mathrm{H})$ \\
\hline 1630 & 1630 & 1630 & $\mathrm{H}-\mathrm{O}-\mathrm{H}$ \\
\hline 1456 & 1456 & 1452 & $\delta(\mathrm{C}-\mathrm{C})$ \\
\hline 1378 & 1373 & 1380 & $\delta(\mathrm{C}-\mathrm{H})$ \\
\hline 1058 & 1058 & 1040 & $\nu(\mathrm{C}-\mathrm{O})+\nu(\mathrm{C}-\mathrm{N})$ \\
\hline 898 & 890 & 882 & $\nu(\mathrm{C}-\mathrm{N})+\nu(\mathrm{C}-\mathrm{C})+\nu(\mathrm{C}-\mathrm{O})$ \\
\hline
\end{tabular}

Band assignments show a significant absorbance in the region between 3500 $\mathrm{cm}^{-1}$ and $3000 \mathrm{~cm}^{-1}$ which is due to the strong $\mathrm{O}-\mathrm{H}$ stretching vibrations and physisorbed and chemisorbed $\mathrm{H}_{2} \mathrm{O}$. The $2830 \mathrm{~cm}^{-1}$ and $2950 \mathrm{~cm}^{-1}$ bands cor- 
respond to the $\mathrm{C}-\mathrm{H}$ stretching vibrational mode. The $1620 \mathrm{~cm}^{-1}$ band is due to H-O-H bending of $\mathrm{H}_{2} \mathrm{O}$ molecules. The bands in the region of $1160-1460 \mathrm{~cm}^{-1}$ are associated with vibrations of the $\mathrm{C}-\mathrm{C}$ and $\mathrm{C}-\mathrm{H}$ bonds. The $\mathrm{C}-\mathrm{O}$ and $\mathrm{C}-\mathrm{N}$ stretching vibrations were detected at $1050 \mathrm{~cm}^{-1}$. The band at $890 \mathrm{~cm}^{-1}$ could be due to C-N, C-C, C-O and C-C-O vibrations.

The as synthesized SnS nanoparticles are readily dispersed in water for biomedical applications and other polar solvents for the incorporation of the IR-sensitive SnS nanoparticles into biphasic polymer blends for photovoltaic devices.

\subsubsection{Experiment $\mathrm{D}$ : Effect of injection speed of $\mathrm{Na}_{2} \mathrm{~S}$}

As described above, monodisperse SnS nanoparticles have been synthesized at room temperature by dropwise addition of $\mathrm{Na}_{2} \mathrm{~S}$ solution into the mixture of $\mathrm{SnBr}_{2}$ and TEA ethylene glycol solution. It is known that the concentration of precursor may affect the morphology of the products. ${ }^{46}$ In the present system, the concentration of $\mathrm{S}^{2-}$ ions is dependent upon the injection speed of $\mathrm{Na}_{2} \mathrm{~S}$ solution. Hence, it is interesting to understand the effect of injection speed of $\mathrm{Na}_{2} \mathrm{~S}$ on the size of the produced product. The following experiment was carried out using rapid rather than dropwise injection, keeping the other reaction conditions same as that in the Experiment $\mathrm{C}$.

\section{Results and discussion}

The as synthesized nanoparticles are shown in Fig. 5.8a with size distribution analysis shown in Fig. 5.8b. The nanoparticles have irregular shapes and are $5.0 \pm 2.0 \mathrm{~nm}$ in size. 

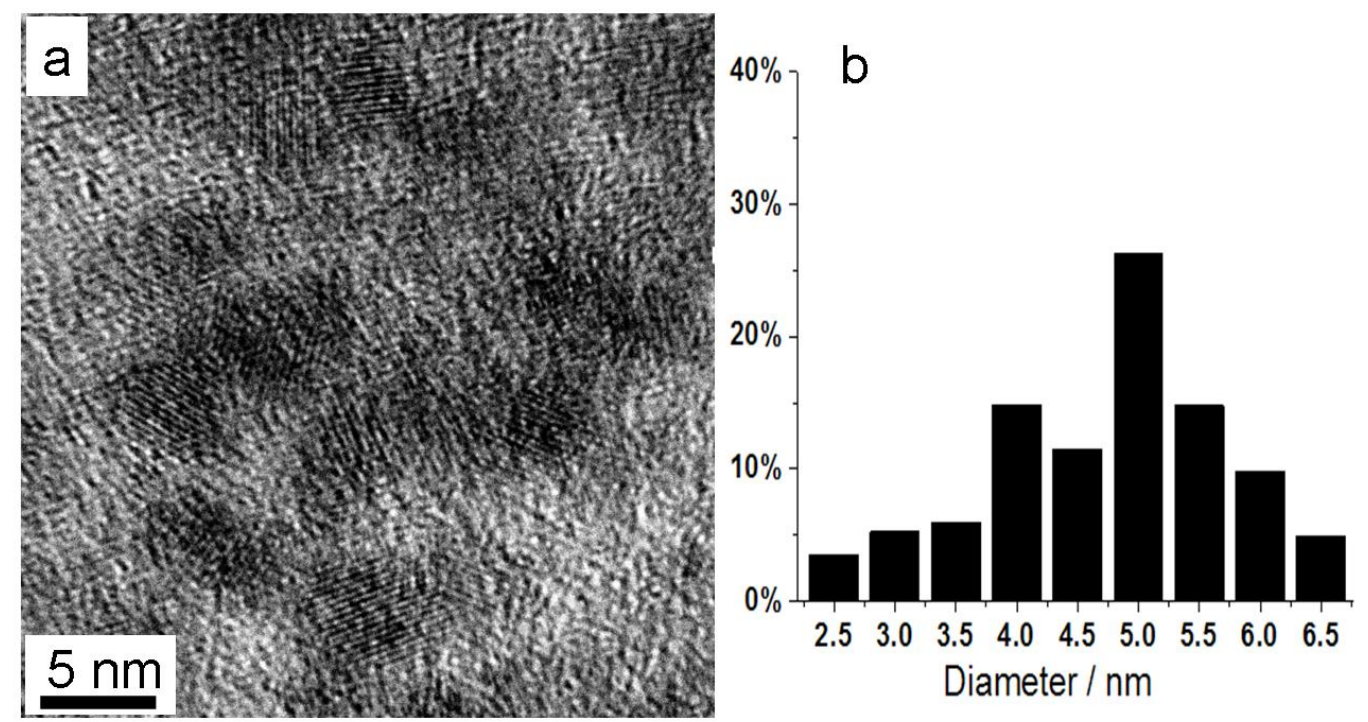

Fig. 5.8 (a) TEM images of SnS nanoparticles obtained through swift injection of $\mathrm{Na}_{2} \mathrm{~S}$ and (b) histogram of nanoparticle size distribution.

The results, as shown in Table 5.4, indicate that the size of SnS nanoparticles increase with the increasing concentration of $\mathrm{S}^{2-}$ ions via rapid injection of $\mathrm{Na}_{2} \mathrm{~S}$. $\mathrm{Na}_{2} \mathrm{~S}$ is a very reactive sulphur precursor which can easily release $\mathrm{S}^{2-}$ ions. ${ }^{36}$ In the reaction system, $\mathrm{Sn}^{2+}$ is the rate determinant, which depends on the stability of intermediate tin complex, which determined how many $\mathrm{Sn}^{2+}$ cations available for reaction with $\mathrm{S}^{2-}$ ions. Quick injection $\mathrm{Na}_{2}$ Sproduce relatively larger and broad size distribution of SnS nanoparticles due to aggregation of small nuclei into larger particles.

Table 5.4 Results for SnS nanoparticles obtained with dropwise or rapid injection of $\mathrm{Na}_{2} \mathrm{~S}$ solution.

\begin{tabular}{|c|c|c|c|c|c|}
\hline Exp & Ethanolamine & Volume $(\mathrm{mL})$ & Rxn Temp & Injection & products \\
\hline C & TEA & 4 & RT & dropwise & $3.2 \pm 0.5 \mathrm{~nm}$ \\
\hline D & TEA & 4 & RT & rapid & $5.0 \pm 2.0 \mathrm{~nm}$ \\
\hline
\end{tabular}

Fig. 5.9 shows the reflectivity data for films of the dropwise and rapid injection nanoparticle samples. The large particles produced using rapid injection show a reflectivity edge that is red-shifted by $\sim 0.4 \mathrm{eV}$ compared to the dropwise injection sample. The inset plot shows the first derivative of reflectivity with 
respect to incident photon energy, hv; peaks in these data correspond to the reflectivity edge of the semiconductor nanocrystals. The rapid injection sample exhibits a reflectivity edge of $\sim 1.29 \mathrm{eV}$. This value is close to the $1.1 \mathrm{eV}$ reported for the bulk band gap of SnS while lower than $1.65 \mathrm{eV}$ obtained for dropwise injection nanoparticles. ${ }^{11,43}$ The observed size-dependent blue shift in the reflectance edges can be expected as a consequence of quantum confinement effects. The dropwise injection sample has smaller particle size than the rapid injection sample exhibiting an increment on the band gap value which further confirms that, in the present approach, the reflectance edges of SnS nanoparticles can be tuned by the injection speed of sulphur precursor.

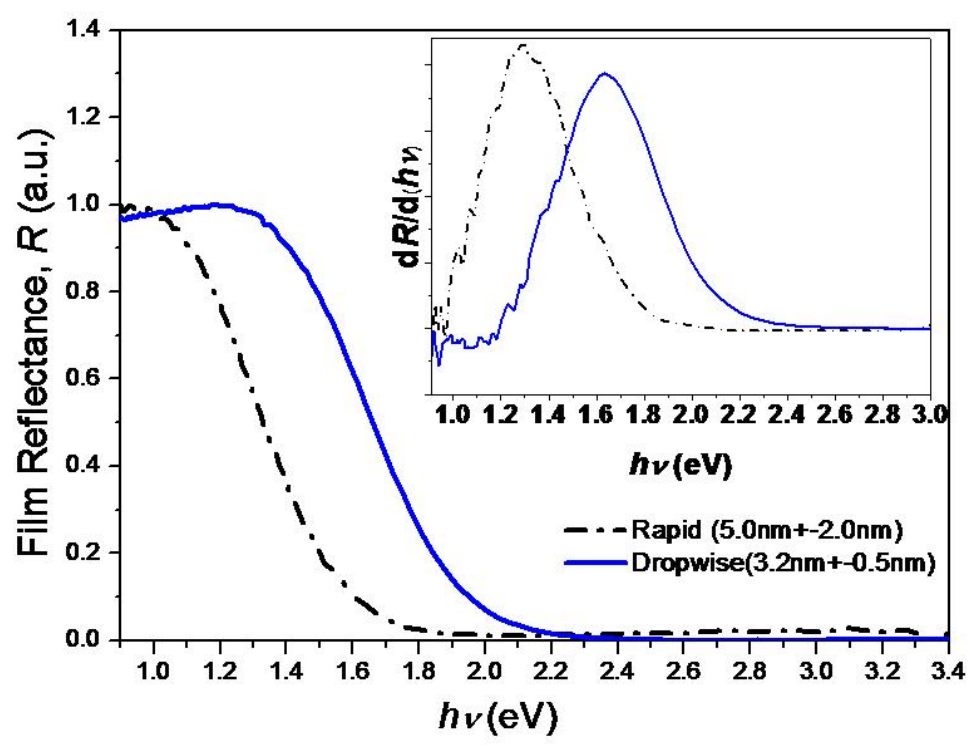

Fig. 5.9 Optical reflectance spectra for film of SnS nanoparticles obtained via rapid and dropwise injection. Inset: First derivative of film reflectance vs incident photon energy.

\subsubsection{Experiment E-G : Effect of the amount of TEA}

In the reaction system, TEA may serve a dual role, as both a chelate ligand and surfactant. Hence, it is important to understand the optimal amount of TEA required in the reaction. Apart from $4 \mathrm{~mL}$ TEA used in experiment $\mathrm{C}$, two 
additional experiments were carried out using $2.0 \mathrm{~mL}$ (Experiment E) and 6.0 $\mathrm{mL}$ (Experiment F) TEA. For comparison, a further experiment was performed without TEA present (Experiment G).

\section{Results and discussion}

Fig. 5.10 illustrates TEM images of samples prepared in the presence of 2.0 and 6.0 mL of TEA. When $2.0 \mathrm{~mL}$ TEA was used, the nanoparticles have a roughly spherical or oval shape and are $4.0 \pm 1.5 \mathrm{~nm}$ in size (Fig. 5.10b). When $6.0 \mathrm{~mL}$ TEA was used in the reaction, roughly spherical nanoparticles of average size of $3.0 \pm 0.5 \mathrm{~nm}$ were formed (Fig. 5.10c,d).
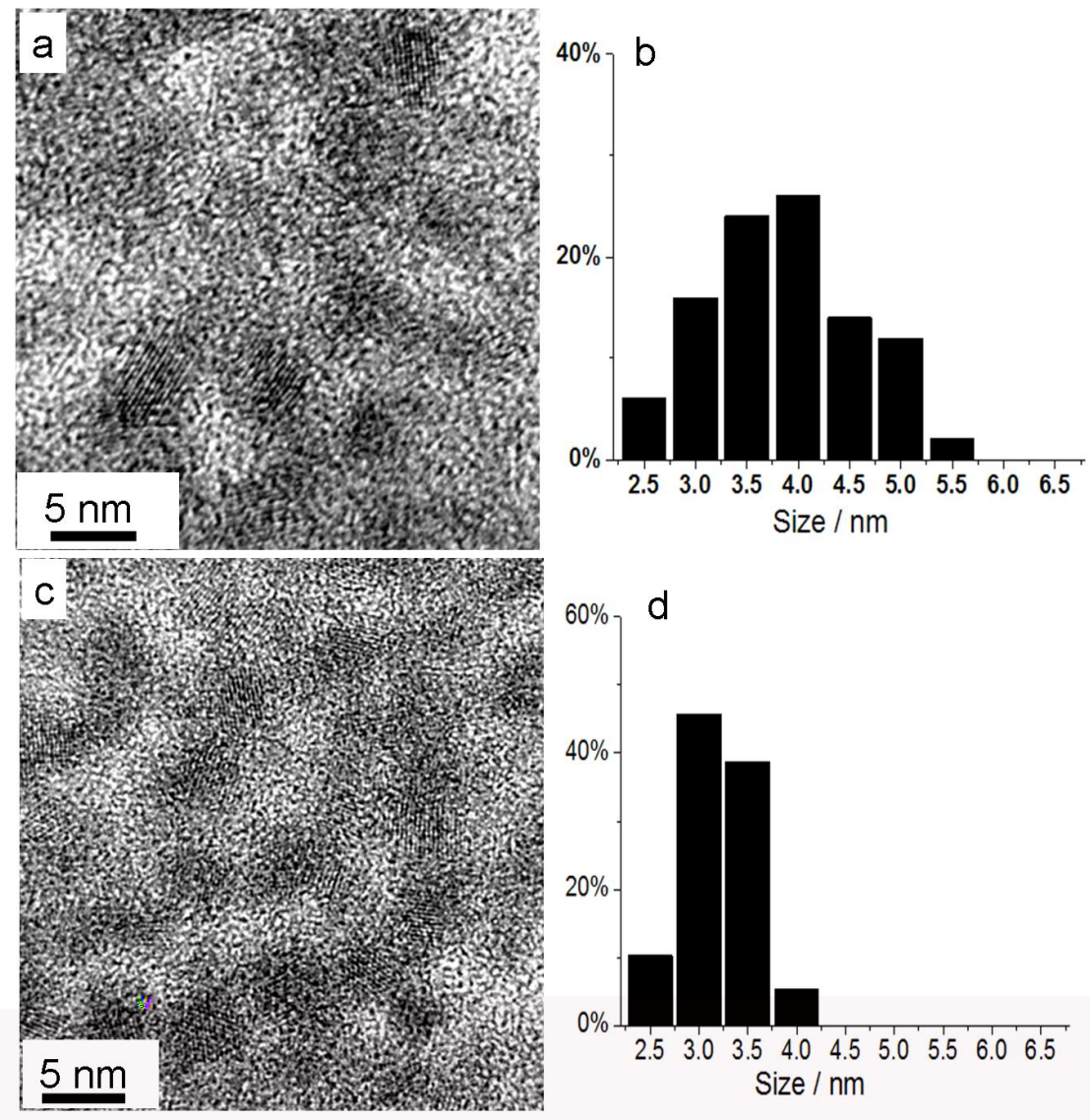

Fig. 5.10 HRTEM images of SnS nanoparticles synthesized in the presence of $2 \mathrm{~mL}$ (a) and $6 \mathrm{~mL}$ (c) TEA at room temperature, respectively. The corresponding histogram of size distributions for the SnS nanoparticles showing in (b) and $(\mathrm{d})$ 
The results summarized in Table 5.6 show that the size of SnS nanoparticles reduced with increasing amount of TEA in the reaction system. This is probably due to the fact that TEA coating stabilizes particles so as to obtain SnS particles of less than $6 \mathrm{~nm}$. This reveals that the size of SnS nanoparticles can be controlled by the stabilizing ligand TEA, which can control the growth of SnS nuclei and form nearly uniform particles. Based on the experimental results obtained together with the costs of experiments, $4 \mathrm{~mL}$ TEA is optimum for synthesis of small SnS nanoparticles.

Table 5.5 Results for SnS nanoparticles obtained in the presence of different amount of TEA.

\begin{tabular}{|c|c|c|c|c|c|}
\hline Exp & Ethanolamine & Volume $(\mathrm{mL})$ & Rxn Temp & Injection & products \\
\hline G & TEA & 0 & RT & dropwise & Clusters \\
\hline E & TEA & 2 & RT & dropwise & $4.0 \pm 1.5 \mathrm{~nm}$ \\
\hline C & TEA & 4 & RT & dropwise & $3.2 \pm 0.5 \mathrm{~nm}$ \\
\hline F & TEA & 6 & RT & dropwise & $3.0 \pm 0.5 \mathrm{~nm}$ \\
\hline
\end{tabular}

In contrast, the product obtained without TEA consisted of SnS clusters (Fig. 5.11), which further demonstrated that TEA play a crucial role in the size controlled synthesis of the SnS nanoparticles.

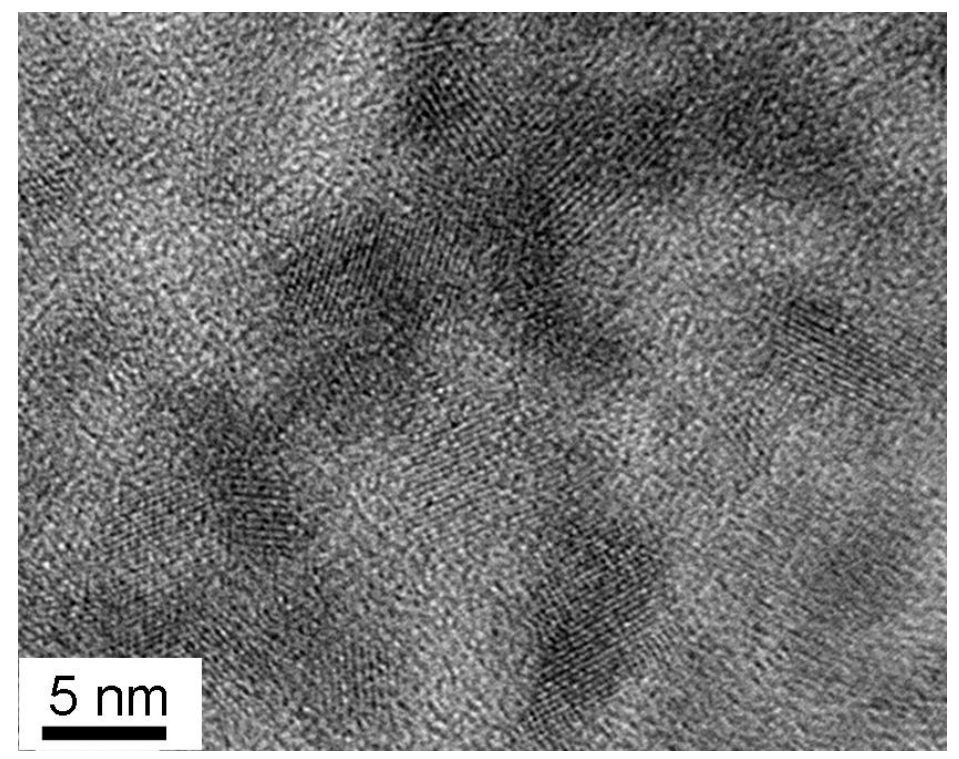

Fig. 5.11 HRTEM image of the sample obtained without TEA. 
Fig. 5.12 shows the diffuse reflectance spectra obtained for films of the samples prepared with $0,2,4$ or $6 \mathrm{~mL}$ of TEA. The samples produced using TEA $(2,4$ or $6 \mathrm{~mL}$ ) show reflectively edges that are blue-shifted compared to the sample prepared without TEA. The inset plot shows the first derivative of reflectivity with respect to incident photon energy, $\mathrm{h} v$; peaks in these data correspond to the reflectivity edge of the semiconductor nanocrystals. The 2,4 and $6 \mathrm{~mL}$ TEA samples exhibit reflective edge of $1.5 \mathrm{eV}, 1.65 \mathrm{eV}$ and $1.7 \mathrm{eV}$, respectively. In contrast, SnS cluster which formed without TEA exhibit a reflective edge of $\sim 1.2 \mathrm{eV}$. These values correspond to particles of different size that depends on the amount of TEA used (Table 5.6). These observations suggest that the reflectance edges of SnS nanoparticles can be tuned by adding TEA surfactant and that the amount of TEA has the ability to control the size of SnS nanoparticles.

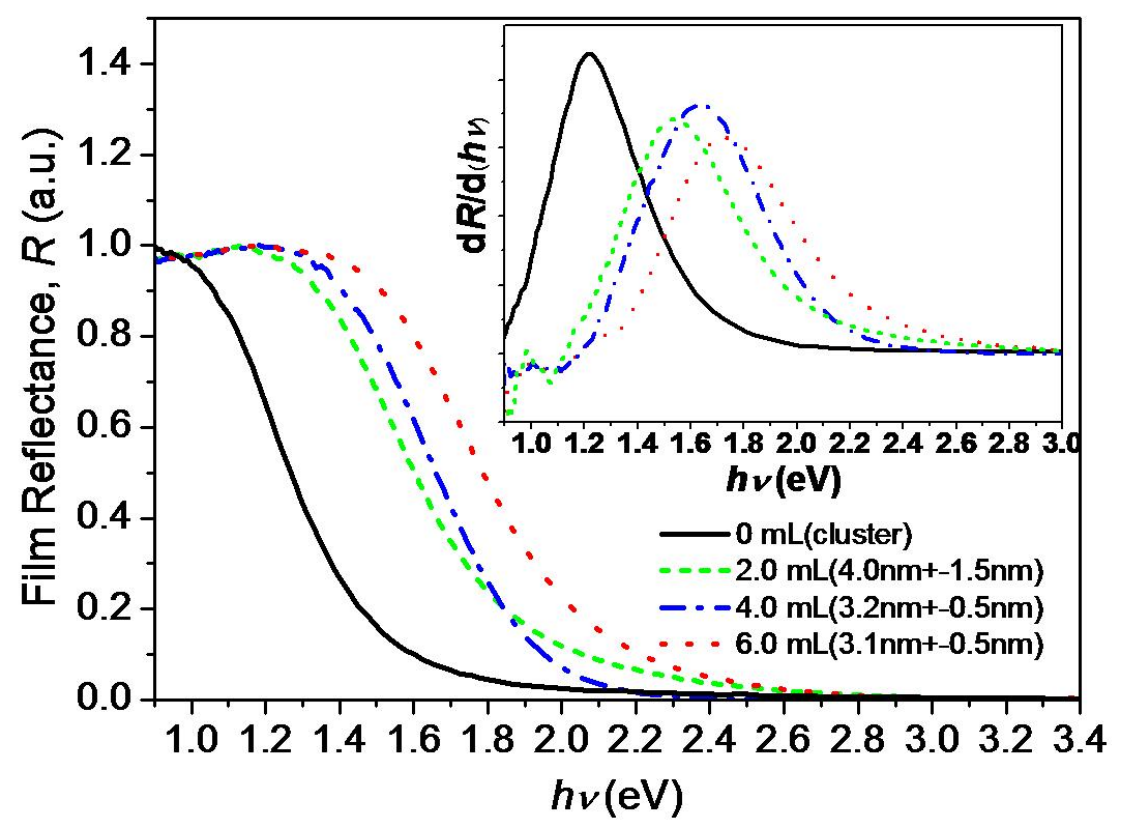

Fig. 5.12 Optical reflectance spectra of SnS nanoparticle films. Inset: First derivative of film reflectance vs incident photon energy. 


\subsubsection{Experiment $\mathrm{H}-\mathrm{I}$ : Effect of the reaction temperature}

It is known that the reaction temperature may play a crucial role in controlling the kinetics and thermodynamics of nucleation/growth of nanoparticle, as well as their optical properties. ${ }^{47,48}$ To probe the effect of temperature on the synthesis and optical properties, two additional experiments were performed at temperatures $0{ }^{\circ} \mathrm{C}$ (experiment $\mathrm{H}$ ) and $100{ }^{\circ} \mathrm{C}$ (experiment $\mathrm{I}$ ), keeping the other reaction conditions same as in experiment $C$.

\section{Results and discussion}

A typical TEM image of the product obtained at $0{ }^{\circ} \mathrm{C}$ is presented in Fig. $5.13 \mathrm{a}$, showing the nanoparticles exhibit clearly resolved lattice fringes indicating the formation of highly crystalline $\mathrm{SnS}$ nanoparticles at $0^{\circ} \mathrm{C}$. A histogram showing the nanoparticles size distribution is shown in Fig. 5.13b, indicating an average nanoparticle size of $4.0 \pm 1.0 \mathrm{~nm}$. Fig. 5.14 shows the product prepared at $100{ }^{\circ} \mathrm{C}$ in which SnS nanoparticles are aggregated forming big clusters.
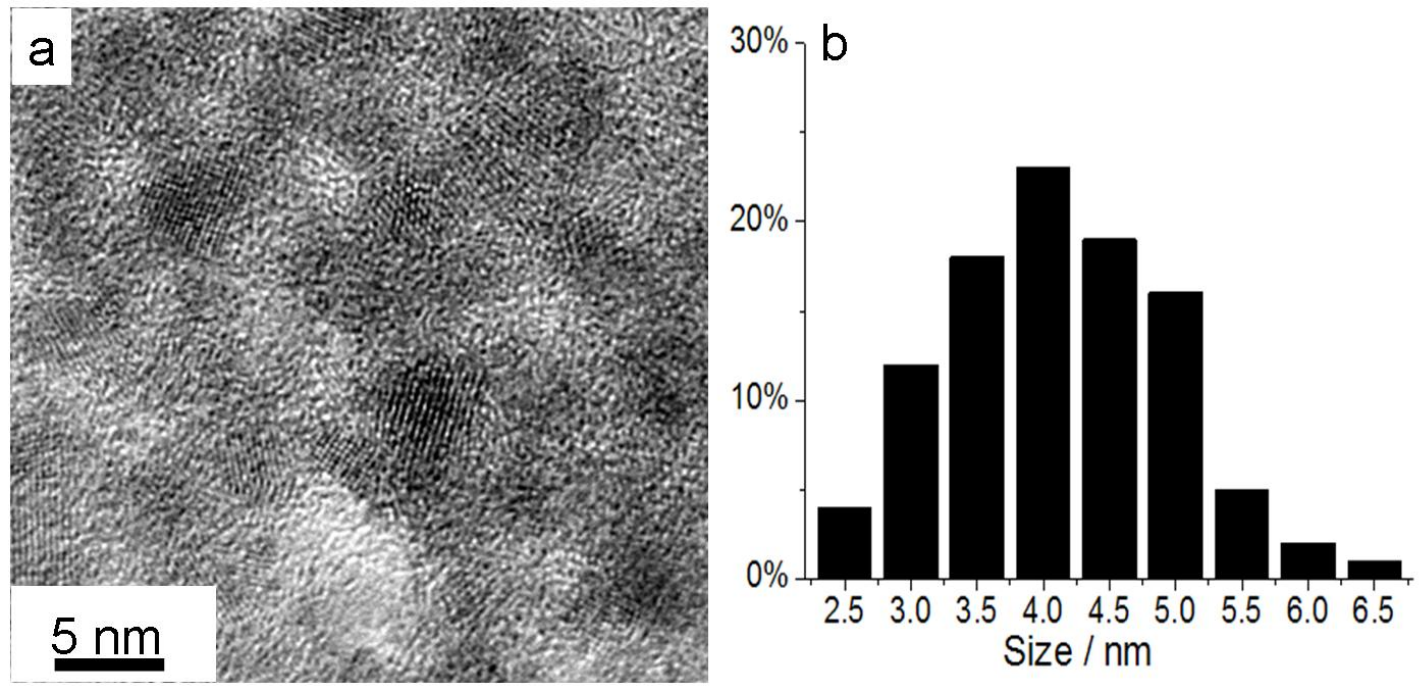

Fig. 5.13 (a)HRTEM images of SnS nanoparticles synthesized in the presence of $4 \mathrm{~mL}$ TEA at $0{ }^{\circ} \mathrm{C}$; (b) the corresponding histogram of size distributions for the $\mathrm{SnS}$ nanoparticles 


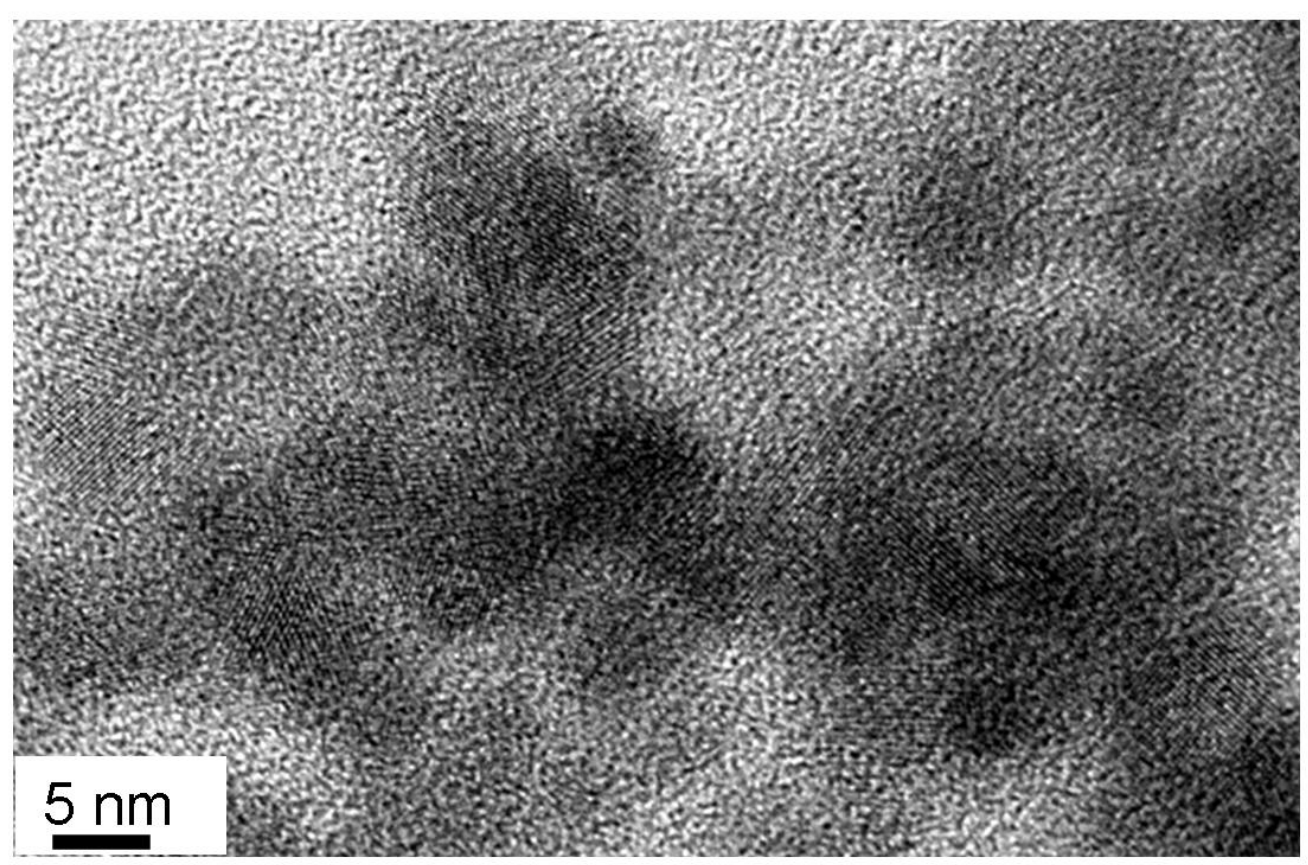

Fig. 5.14 A typical HRTEM images of SnS cluster synthesized in the presence of $4 \mathrm{~mL}$ TEA at $100{ }^{\circ} \mathrm{C}$.

Comparing the results obtained from different temperatures (Table 5.6), it was found that there is only a small difference in the average size and the size distribution between the products formed at $0{ }^{\circ} \mathrm{C}$ and at room temperature. The RT sample exhibits a smaller size and relatively narrow size distribution than the $0{ }^{\circ} \mathrm{C}$ sample. This may be explained by that increasing the reaction temperature enhanced the number nuclei for subsequent growth and would reduce the size of nanoparticles. ${ }^{49,50}$ However, when the synthesis was performed at $100{ }^{\circ} \mathrm{C}$, big SnS clusters were formed. The experimental results suggest that the stability of the intermediate tin complex is temperature dependent. At $100{ }^{\circ} \mathrm{C}$, the tin complex becomes more reactive and forms clusters, as compared to room temperature reactions where nanoparticles are formed. .

Table 5.6 Results for SnS nanoparticles prepared at different temperature.

\begin{tabular}{|c|c|c|c|c|c|}
\hline Exp & Ethanolamine & Volume $(\mathrm{mL})$ & Rxn Temp & Injection & products \\
\hline H & TEA & 4 & $0{ }^{\circ} \mathrm{C}$ & dropwise & $4.0 \pm 1.0 \mathrm{~nm}$ \\
\hline C & TEA & 4 & RT & dropwise & $3.2 \pm 0.5 \mathrm{~nm}$ \\
\hline I & TEA & 4 & $100{ }^{\circ} \mathrm{C}$ & dropwise & cluster \\
\hline
\end{tabular}


Diffuse reflectivity spectra for films of the samples prepared at $0{ }^{\circ} \mathrm{C}$, RT and $100{ }^{\circ} \mathrm{C}$ are shown in Fig. 5.15. Reflectivity edges of the $0{ }^{\circ} \mathrm{C}$ and RT samples are $\sim 1.65 \mathrm{eV}$ which is significantly higher than $1.2 \mathrm{eV}$ obtained for $100{ }^{\circ} \mathrm{C}$ sample.

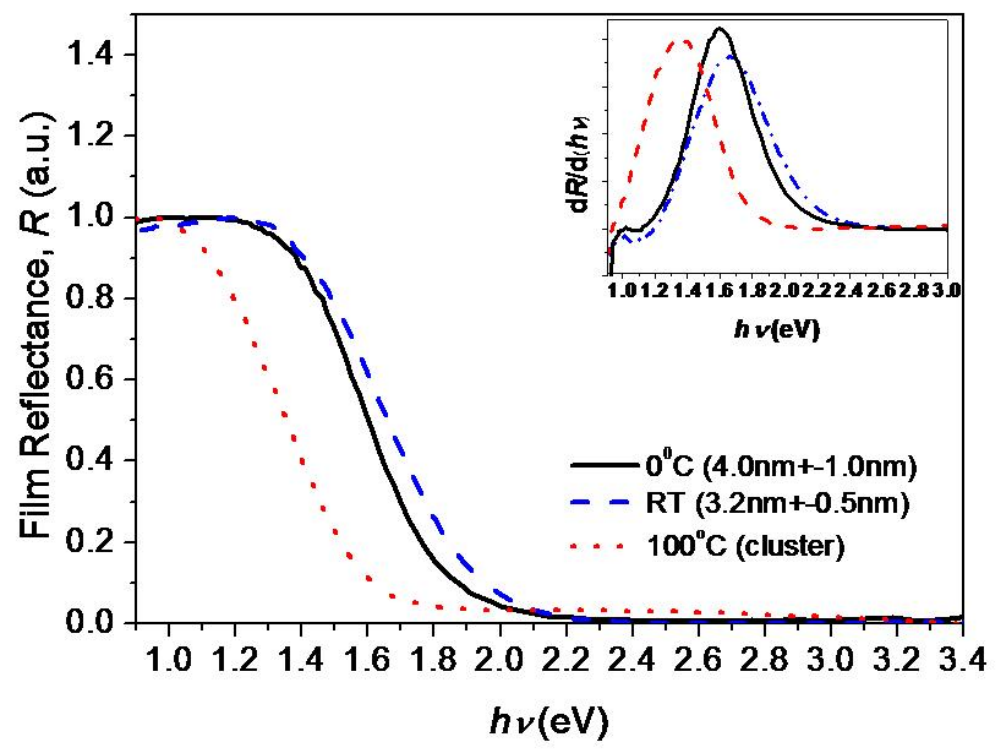

Fig. 5.15 Optical reflectance spectra of SnS nanoparticle films. Inset: First derivative of film reflectance vs incident photon energy.

\subsubsection{Experiment J-L : Effect of high temperature time series}

The aim of the following experiments was to make bigger SnS nanoparticles of various sizes. Since the reaction at $100{ }^{\circ} \mathrm{C}$ produced $\mathrm{SnS}$ clusters rather than individual large SnS nanoparticles, in this series of experiments the synthesis was first carried out at room temperature followed by heating at $100{ }^{\circ} \mathrm{C}$ for different periods of time. This would be expected to form SnS nanoparticles of variable size and size distributions as a result of Ostwald ripening. ${ }^{51}$ These experiments were carried out under the same conditions as in the experiment $\mathrm{C}$, followed by further heating to $100{ }^{\circ} \mathrm{C}$ for $10 \mathrm{~min}$ (experiment J), $30 \mathrm{~min}$ (experiment $\mathrm{K}$ ) and $60 \mathrm{~min}($ experiment $\mathrm{L}$ ), respectively. 


\section{Results and discussion}

Fig. 5.16 shows the TEM images of as synthesized products by heat treatment at $100{ }^{\circ} \mathrm{C}$ for $10 \mathrm{~min}, 30 \mathrm{~min}$ and $60 \mathrm{~min}$. The particles with average size in 4.0 $\pm 1.0 \mathrm{~nm}, 5.5 \pm 1.0 \mathrm{~nm}$ and $6.0 \pm 1.5 \mathrm{~nm}$ were obtained, respectively.
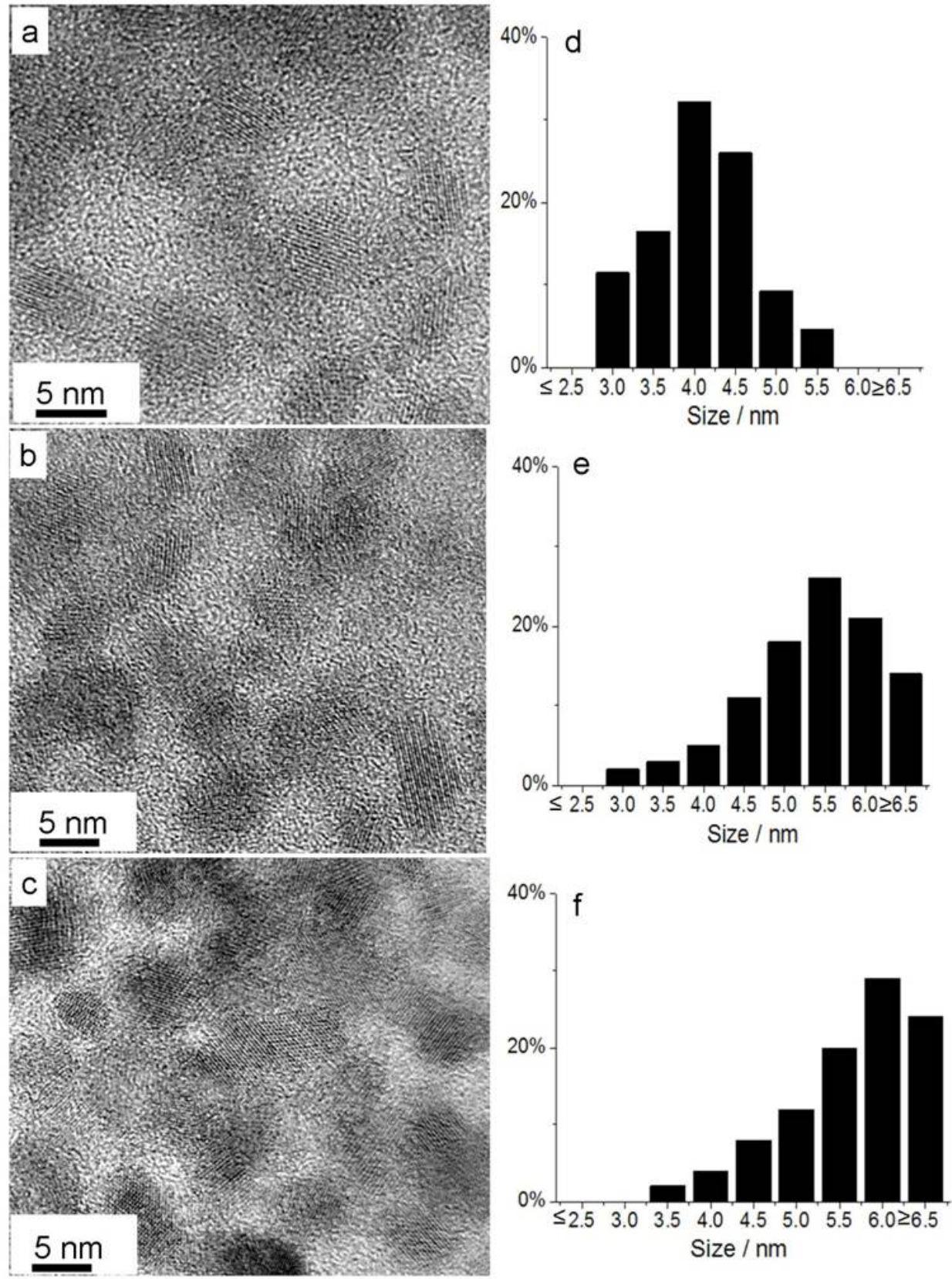

Fig. 5.16 HRTEM images of SnS nanoparticles synthesized at room temperature followed by heating to $100{ }^{\circ} \mathrm{C}$ for 10 (a), 30 (b) and 60 (c) mins, respectively and their corresponding histogram of size distributions for the SnS nanoparticles. 
Based on the results summarized in Table 5.7, it was found that the size of the SnS nanoparticle significantly increased from $3.2 \pm 0.5 \mathrm{~nm}$ to $6.0 \pm 1.5 \mathrm{~nm}$ by further heating of the nanoparticles at $100{ }^{\circ} \mathrm{C}$ for between 10 and 60 minutes. The particle size distribution becomes slightly broader during this period. This indicates that small spherical colloidal SnS nanoparticles were first formed and stabilized by TEA at room temperature, and then by further heating to $100{ }^{\circ} \mathrm{C}$ the size distribution of the SnS-TEA system was shifted. Owing to Ostwald ripening, bigger particles tend to grow and smaller particles tend to shrink in the system. As a consequence, the average SnS particle size increased and the size distribution because broader with the time. In addition, large SnS nanoparticles exhibit oval or irregular shape which may be due to its high anisotropic (orthorhombic) crystal structure. Similar irregularly shaped SnS nanoparticles were also reported in the literature. ${ }^{17}$

Table 5.7 Results for SnS nanoparticles obtained with or without further heat treatment.

\begin{tabular}{|c|c|c|c|c|c|}
\hline Exp & Surfactant & Vol $(\mathrm{mL})$ & Rxn Temp & Injection & products \\
\hline C & TEA & 4 & RT & dropwise & $3.2 \pm 0.5 \mathrm{~nm}$ \\
\hline J & TEA & 4 & $\mathrm{RT}, 100^{\circ} \mathrm{C}$ for $10 \mathrm{~min}$ & dropwise & $4.0 \pm 1.0 \mathrm{~nm}$ \\
\hline K & TEA & 4 & $\mathrm{RT}, 100^{\circ} \mathrm{C}$ for $30 \mathrm{~min}$ & dropwise & $5.5 \pm 1.0 \mathrm{~nm}$ \\
\hline L & TEA & 4 & $\mathrm{RT}, 100^{\circ} \mathrm{C}$ for $60 \mathrm{~min}$ & dropwise & $6.0 \pm 1.5 \mathrm{~nm}$ \\
\hline
\end{tabular}

Diffuse reflectance spectra for SnS films of the samples prepared with or without heat treatment is shown in Fig. 5.17. The smaller sized particles produced at room temperature and heated at $100{ }^{\circ} \mathrm{C}$ for $10 \mathrm{~min}$ shows a reflectivity edge that is blue shifted by $\sim 0.1 \mathrm{eV}$ or $\sim 0.2 \mathrm{eV}$ compared to the particles heated for $30 \mathrm{~min}$ or $60 \mathrm{~min}$, respectively. The inset plot shows the first derivative of reflectivity with respect to incident photo energy, $\mathrm{h} v$. The RT and heated for 10 min samples exhibit reflectivity edges of $\sim 1.65 \mathrm{eV}$. The $10 \mathrm{~min}$ sample shows a relatively broader peak than the RT sample, which may be attributed to its broader particle size distribution. In contrast the $30 \mathrm{~min}$ and $60 \mathrm{~min}$ samples 
exhibit peaks in the reflectance derivative at $\sim 1.55 \mathrm{eV}$ and $\sim 1.45 \mathrm{eV}$, respectively. These results demonstrate that the reflectivity edge of nanoparticles depends strongly on the particle size and size distribution.

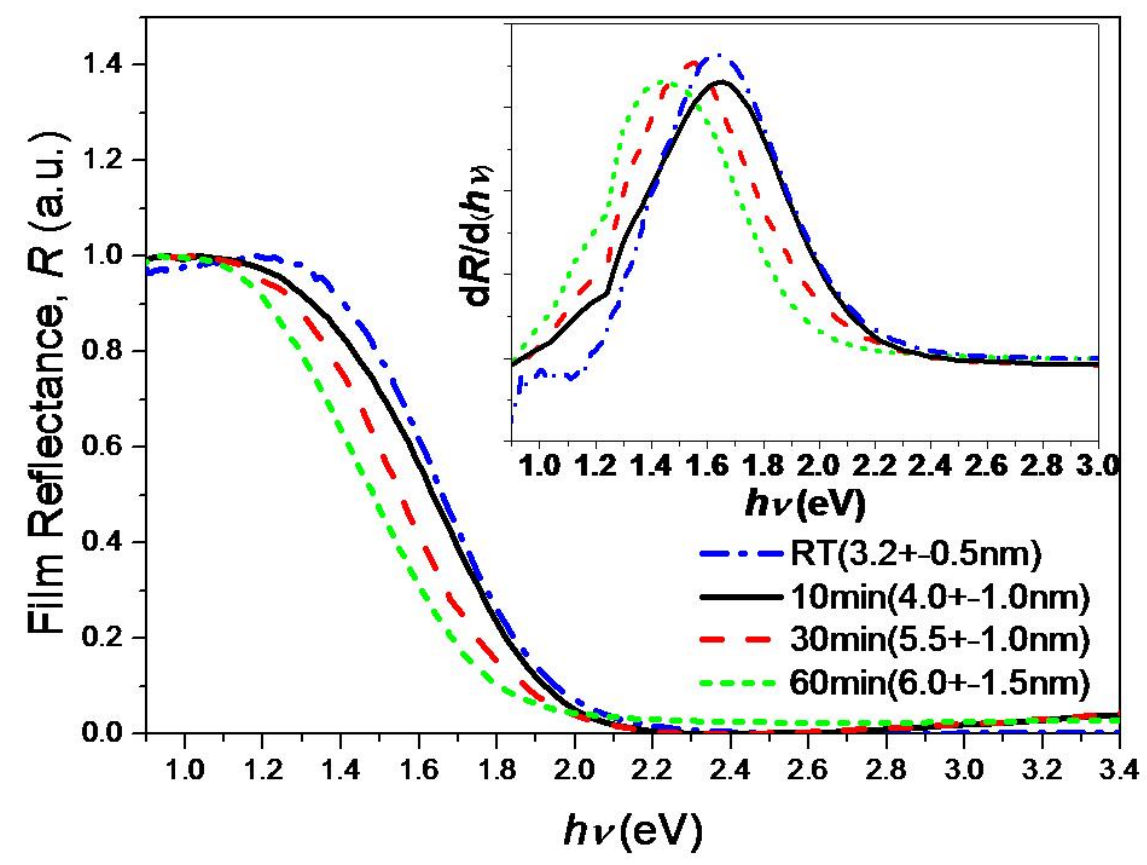

Fig. 5.17 Optical reflectance spectra of SnS nanoparticle films. Inset: First derivative of film reflectance vs incident photon energy.

\subsection{Summary}

In this chapter, the synthesis of SnS nanoparticles through facile, inexpensive and straightforward methods has been developed. Most of syntheses were carried out at room temperature and the chemicals used were environmentally friendly. All the samples can be dispersed in water.

Tertiary ethanolamines with hydroxyl groups, were demonstrated to be suitable surfactants to directly synthesize water-soluble SnS nanoparticles. A comparison of the results obtained from different ethanolamine derivatives, in- 
dicates the crucial role of the surfactants in the synthesis of size controllable SnS nanoparticles. TEA exhibits best size control, then MDEA followed by DMEA. This demonstrates the effect of the number of hydroxyl groups in the surfactant molecules on size control of SnS nanoparticles. The observed size-dependent optical properties of the SnS nanoparticles further proved that surfactant is a very important factor in the synthesis and properties of SnS nanoparticles.

$\mathrm{Na}_{2} \mathrm{~S}$ is a commonly used sulphur precursor in aqueous solution, which can easily release $\mathrm{S}^{2-}$ ions. The injection speed of $\mathrm{Na}_{2} \mathrm{~S}$ affects the morphology of final particles. Experimental results demonstrated that the dropwise injection technique led to formation of monodispersed SnS nanoparticles.

Morphologically, the amount of TEA present is also significantly important as the effect of the type of ethanolamine derivatives for the synthesis of size controllable SnS nanoparticles, with the optimal value of TEA needed being 4 $\mathrm{mL}$ in $20 \mathrm{~mL}$ of solvent.

In addition, the size of the SnS nanoparticles can be controllably varied from $3 \mathrm{~nm}$ up to $6 \mathrm{~nm}$ by further heating of the nanoparticles at $100{ }^{\circ} \mathrm{C}$ for different length of time between 10 and 60 minutes. The resulting SnS nanoparticles exhibit a blue shift in reflectivity edge with decreasing particle size, mainly due to the quantum confinement effect. ${ }^{42,52}$

In summary, colloidal SnS nanoparticles with a size of a few nanometers have been synthesized using ethanolamine ligands. The size of the resulting SnS nanoparticles can be tuned by varying the reaction parameters. Surfactants were shown to be the key factor in the synthesis of size controlled SnS nanopar- 
ticles. TEA, was an excellent candidate of surfactant, as it restricted the growth of SnS nanoparticles to provide uniform particles of $4 \mathrm{~nm}$. TEA may be potentially used in synthesizing other monodispersed water soluble tin chalcogenide nanoparticles. This prompted us to investigate formation of SnTe nanoparticles as described in Chapter 6. 


\section{REFERENCES}

[1] Chamberlain, J. M.; Nikolic, P. M.; Merdan, M.; Mihailovic, P. Journal of Physics C:Solid State Physics 1976, 9, 637-642.

[2] Souici, A. H.; Keghouche, N.; Delaire, J. A.; Remita, H.; Etcheberry, A.; Mostafavi, M. The Journal of Physical Chemistry C 2009, 113, 8050-8057.

[3] Wang, Y.; Gong, H.; Fan, B.; Hu, G. The Journal of Physical Chemistry C 2010, $114,3256-3259$.

[4] Kovalenko, M. V.; Heiss, W.; Shevchenko, E. V.; Lee, J.-S.; Schwinghammer, H.; Alivisatos, A. P.; Talapin, D. V. Journal of the American Chemical Society 2007, 129, 11354-11355.

[5] Washington Ii, A. L.; Strouse, G. F. Journal of the American Chemical Society 2008, 130, 8916-8922.

[6] Al-Salim, N.; Young, A. G.; Tilley, R. D.; McQuillan, A. J.; Xia, J. Chemistry of Materials 2007, 19, 5185-5193.

[7] Ouyang, J.; Vincent, M.; Kingston, D.; Descours, P.; Boivineau, T.; Zaman, M. B.; Wu, X.; Yu, K. The Journal of Physical Chemistry C 2009, 113, 5193-5200.

[8] Chen, F.; Zhou, R.; Yang, L.; Shi, M.; Wu, G.; Wang, M.; Chen, H. The Journal of Physical Chemistry C 2008, 112, 13457-13462.

[9] Liu, J.; Yu, H.; Wu, Z.; Wang, W.; Peng, J.; Cao, Y. Nanotechnology 2008, 19, 345602.

[10] Jiang, T.; Ozin, G. A.; Verma, A.; Bedard, R. L. Journal of Materials Chemistry 1998, 8, 1649-1656. 
[11] Thangaraju, B.; Kaliannan, P. Journal of Physics D: Applied Physics 2000, 33, 1054.

[12] Ortiz, A.; Alonso, J. C.; Garcia, M.; Toriz, J. Semiconductor Science and Technology 1995, 243247.

[13] Tanusevski, A.; Poelman, D. Solar Energy Materials and Solar Cells 2003, 80, 297-303.

[14] Gou, X.-L.; Chen, J.; Shen, P.-W. Materials Chemistry and Physics 2005, 93, $557-566$.

[15] Schlecht, S.; Kienle, L. Inorganic Chemistry 2001, 40, 5719-5721.

[16] Koktysh, D. S.; McBride, J. R.; Rosenthal, S. J. Nanoscale Research Letter $2007,2,144-148$.

[17] Hickey, S. G.; Waurisch, C.; Rellinghaus, B.; Eychmuller, A. Journal of the American Chemical Society 2008, 130, 14978-14980.

[18] Liu, H.; Liu, Y.; Wang, Z.; He, P. Nanotechnology 2010, 21, 105707.

[19] Mrinmoy, D.; Partha, S. G.; Vincent, M. R. Advanced Materials 2008, 20, $4225-4241$.

[20] Chan, W. C. W.; Nie, S. Science 1998, 281, 2016-2018.

[21] Marcel Bruchez, J.; Moronne, M.; Gin, P.; Weiss, S.; Alivisatos, A. P. Science $1998,281,2013-2016$.

[22] Noh, M.; Kim, T.; Lee, H.; Kim, C.-K.; Joo, S.-W.; Lee, K. Colloids and Surfaces A: Physicochemical and Engineering Aspects 2010, 359, 39 - 44.

[23] Winter, J. O.; Liu, T. Y.; Korgel, B. A.; Schmidt, C. E. Advanced Materials 2001, 13, 1673-1677. 
[24] Purkayastha, A.; Kim, S.; Gandhi, D. D.; Ganesan, P. G.; Borca-Tasciuc, T.; Ramanath, G. Advanced Materials 2006, 18, 2958-2963.

[25] Pathak, S.; Choi, S.-K.; Arnheim, N.; Thompson, M. E. Journal of the American Chemical Society 2001, 123, 4103-4104.

[26] Algar, W. R.; Ulrich, J. K. ChemPhysChem 2007, 8, 561-568.

[27] Jing, S.; Shaobing, Z.; Peng, H.; Yuan, Y.; Jie, W.; Xiaohong, L.; Mingyuan, L. Journal of Biomedical Materials Research Part A 2007, 80A, 333341.

[28] Li, Z.; Chen, H.; Bao, H.; Gao, M. Chemistry of Materials 2004, 16, 13911393.

[29] Biswas, S.; Kar, S.; Chaudhuri, S. Applied Surface Science 2007, 253, 9259 9266.

[30] Peng, H.; Jiang, L.; Huang, J.; Li, G. Journal of Nanoparticle Research 2007, 9, 1163-1166.

[31] El-Sayed, M. A. Accounts of Chemical Research 2004, 37, 326-333.

[32] Jun, Y.-w.; Lee, J.-H.; Choi, J.-s.; Cheon, J. The Journal of Physical Chemistry B 2005, 109, 14795-14806.

[33] Somasundaran, P. Encyclopedia of Surface And Colloid Science; CRC Press; 2 edition, 2006; Vol. 6, p 4783.

[34] Pramanik, P.; Basu, P. K.; Biswas, S. Thin Solid Films 1987, 150, 269-276.

[35] An, C.; Tang, K.; Shen, G.; Wang, C.; Yang, Q.; Hai, B.; Qian, Y. Journal of Crystal Growth 2002, 244, 333-338.

[36] Tang, H.; Yu, J.; FZhao, X. Journal of Alloys and Compounds 2008, 460, 513518. 
[37] Liu, Y.; Hou, D.; Wang, G. Chemical Physics Letters 2003, 379, 67 - 73.

[38] Zhang, J.; An, M.; Chang, L. Electrochimica Acta 2009, 54, 2883 - 2889.

[39] Sen, B.; Dotson, R. L. Journal of Inorganic and Nuclear Chemistry 1970, 32, $2707-2716$.

[40] Hu, Y. h.; Lei, T. m.; Li, H. s.; Zhang, C. r. Journal of Xi'an University of Technology 2004, 20, 392-395.

[41] Albers, W.; Haas, C.; van der Maesen, F. Journal of Physics and Chemistry of Solids 1960, 15, 306-310.

[42] Hagfeldt, A.; Graetzel, M. Chemical Reviews 1995, 95, 49-68.

[43] Li, X.; Li, J.-G.; Huo, D.; Xiu, Z.; Sun, X. The Journal of Physical Chemistry C 2009, 113, 1806-1811.

[44] Pradhan, D.; Leung, K. T. Langmuir 2008, 24, 9707-9716.

[45] Bumby, C. W. The calculation was thankfully done by Chris W. Bumby; Technical Report, 2009.

[46] Zhang, L.-J.; Shen, X.-C.; Liang, H.; Guo, S.; Liang, Z.-H. Journal of Colloid and Interface Science 2010, 342, 236 - 242.

[47] Wang, C.; Li, Y. D.; Zhang, G. H.; Zhuang, J.; Shen, G. Q. Inorganic Chemistry 2000, 39, 4237-4239.

[48] Wu, P.-Y.; Pike, J.; Zhang, F.; Chan, S.-W. International Journal of Applied Ceramic Technology 2006, 3, 272-278.

[49] Zhu, H.; Zhang, C.; Yin, Y. Nanotechnology 2005, 16, 3079.

[50] Goy-Lopez, S.; Taboada, P.; Cambon, A.; Juarez, J.; Alvarez-Lorenzo, C.; Concheiro, A.; Mosquera, V. The Journal of Physical Chemistry B 2009, 114, 66-76. 
[51] Redmond, P. L.; Hallock, A. J.; Brus, L. E. Nano Letters 2004, 5, 131-135.

[52] Zhang, Y.; He, J.; Wang, P.-N.; Chen, J.-Y.; Lu, Z.-J.; Lu, D.-R.; Guo, J.; Wang, C.-C.; Yang, W.-L. Journal of the American Chemical Society 2006, 128, 13396-13401. 


\section{Chapter 6}

\section{Synthesis of Tin Telluride Nanoparticles}

\section{Contents}

6.1 Introduction . . . . . . . . . . . . . 163

6.2 Synthesis of SnTe nanoparticles in DMF . . . . . . . 168

6.3 Synthesis of SnTe nanoparticles in $\mathrm{H}_{2} \mathrm{O} \ldots \ldots \ldots \ldots$

6.4 Summary. . . . . . . . . . . . . . . . 195

\subsection{Introduction}

This chapter is focused on the solution phase synthesis of water-soluble tin telluride nanoparticles, where a novel one-pot direct synthesis was developed. The synthetic methods described here involve the reaction of tin bromide and sodium hydrogen telluride in the presence of ethanolamine or thio-ligands.

Semiconductor SnTe has attracted considerable interest due to the potential applications in photovoltaic, thermovoltaic and thermoelectric devices because 
of its narrow band gap. The direct band gap of bulk SnTe is $0.18 \mathrm{eV}$ at $300 \mathrm{~K}$ which is narrower than that of the other IV-VI semiconductors such as bulk SnS $(1.3 \mathrm{eV})$ and bulk PbS $(0.41 \mathrm{eV}) .{ }^{1-3}$ Previous research has shown the sizedependent band gap expansion in semiconductors. ${ }^{4-6}$ More recently, Talapin and coworkers reported that the band gap of SnTe can be tuned from $0.18 \mathrm{eV}$ in mid IR region $(3-50 \mu \mathrm{m})$ to $0.8 \mathrm{eV}$ in near IR region $(0.78-3 \mu \mathrm{m})$ by reducing the particle size down to $\sim 4.5 \mathrm{~nm} .{ }^{1}$ Owing to their unique and tunable optical properties, SnTe nanoparticles have recently attracted increasing interest for their potential biomedical applications such as hyperthermal therapy. ${ }^{7}$ The current research was motivated by this potential.

Over the past decades, much attention has been given to the synthesis of metal tellurides with strong and stable fluorescence such as CdTe, PbTe and ZnTe as their potential applications in biolabeling, imaging and sensing. ${ }^{8-11}$ In contrast, research on the synthesis of SnTe nanoparticles with a band gap in the near IR region is still relatively new.

Although several synthetic routes such as mechanical alloying, ${ }^{12}$ molecular beam epitaxy ${ }^{13}$ have recently been developed for the preparation of SnTe films, the synthesis approaches of SnTe nanoparticles with good size control are still few. To date, there are only two reports on the synthesis of SnTe nanoparticles. One was reported by Sabine and coworkers, ${ }^{14}$ where the SnTe nanoparticles with size varied between 15-60 $\mathrm{nm}$ were synthesized through the reaction of tin metal with $\mathrm{Ph}_{2} \mathrm{Te}_{2}$ at $165{ }^{\circ} \mathrm{C}$ for 2 hours in diglyme. The other was reported by Talapin et al., ${ }^{1}$ in which the size of as-prepared SnTe nanoparticles could be well-controlled between $4.5 \mathrm{~nm}$ and $15 \mathrm{~nm}$. The synthesis uses the air sensitive organometallic complex $\mathrm{Sn}\left[\mathrm{N}\left(\mathrm{SiMe}_{3}\right)_{2}\right]_{2}$ as a tin precursor and expensive trioctylphosphine telluride (TOPTe) as a tellurium precursor. Oleylamine 
(OLA) and oleic acid (OA) were used as surfactants and octadecene (ODE) was used as the solvent in the reaction. However, both synthetic approaches render hydrophobic SnTe nanoparticles which may restrict their applications in the biomedical field. ${ }^{15}$ Usually, to render these hydrophobic nanoparticles soluble in aqueous solution, a ligand exchange process is required. ${ }^{16}$ The exchange process is time-consuming and the hydrophilic nanoparticles produced sometimes are not stable enough in aqueous solution. ${ }^{16}$ Hence, an economic, direct synthetic approach producing water-soluble SnTe nanoparticles with narrow size distributions is highly desirable.

Generally, direct synthesis of well-defined, water soluble nanoparticles requires the reactions to be carried out in either polar organic solvents (e.g. ethylene glycol (EG), ${ }^{17}$ tetrahydrofuran (THF), ${ }^{18}$ dimethyl formamide $(\mathrm{DMF})^{19}$ ) or the aqueous phase. ${ }^{20}$ Since the synthetic approach for monodispersed SnS nanoparticle has been successfully developed in EG in the presence of triethanolamine (TEA) (Chapter 5), in this project, a similar synthetic approach will be adopted for SnTe nanoparticle by replacing sulphur precursor for tellurium precursor in DMF.

In parallel with the success of polar organic solvent synthesis approaches, aqueous synthesis of water soluble nanoparticles has also been extensively investigated. In contrast to the SnTe nanoparticles, many methods have been developed to prepare CdTe nanoparticles in water in the presence of various thiol-alkyl acids (RSH) (Scheme 6.1) such as thioglycolic acid (TGA) and 3mercaptopropionic acid (MPA). ${ }^{21-23}$ 
Table 6.1 Summary of the conditions used for the aqueous synthesis of CdTe nanoparticles.

\begin{tabular}{|c|c|c|c|c|c|}
\hline Author & precursors & Surfactant & $\begin{array}{l}\text { Molar ratio of } \\
\mathrm{Cd}: \text { Te : RSH }\end{array}$ & Solvent & $\mathrm{pH}$ \\
\hline Gao et al. ${ }^{21}$ & $\begin{array}{l}\mathrm{Cd}\left(\mathrm{ClO}_{4}\right)_{2} \\
\mathrm{NaHTe}\end{array}$ & TGA & $1: 0.5: 2.4$ & $\mathrm{H}_{2} \mathrm{O}$ & $3.5-5$ \\
\hline Eng et al. ${ }^{22}$ & $\begin{array}{l}\mathrm{CdCl}_{2} \\
\mathrm{NaHTe}\end{array}$ & MPA & $1: 0.5: 2.4$ & $\mathrm{H}_{2} \mathrm{O}$ & 8 \\
\hline Rogach et al. ${ }^{23}$ & $\begin{array}{l}\mathrm{Cd}\left(\mathrm{ClO}_{4}\right)_{2} \\
\mathrm{H}_{2} \mathrm{Te}\end{array}$ & MPA & $1: 0.5: 1.3$ & $\mathrm{H}_{2} \mathrm{O}$ & 12 \\
\hline Rogach et al. ${ }^{23}$ & $\begin{array}{l}\mathrm{Cd}\left(\mathrm{ClO}_{4}\right)_{2} \\
\mathrm{H}_{2} \mathrm{Te}\end{array}$ & TGA & $1: 0.5: 1.3$ & $\mathrm{H}_{2} \mathrm{O}$ & $11.2-11.8$ \\
\hline
\end{tabular}

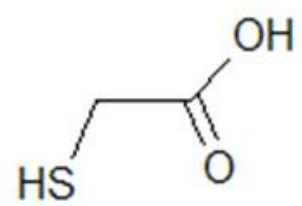

(TGA)<smiles>O=C(O)CCS</smiles>

(MPA)

Scheme 6.1 Molecular structures of TGA and MPA.

As shown in Table 6.1, NaHTe solution and $\mathrm{H}_{2}$ Te gas are commonly used $\mathrm{Te}$ sources for the synthesis of CdTe nanoparticles. These Te precursors are also widely used for preparation of other tellurides such as ZnTe. ${ }^{9} \mathrm{NaHTe}$ solution often prepared by reduction of Te powder with $\mathrm{NaBH}_{4}$ according to the following reactions: ${ }^{24}$

$$
\begin{gathered}
\mathrm{Te}+2 \mathrm{NaBH}_{4} \underset{80^{\circ} \mathrm{C}}{\stackrel{D M F}{\rightarrow}} \mathrm{Na}_{2} \mathrm{Te}+\mathrm{B}_{2} \mathrm{H}_{6}+\mathrm{H}_{2} \\
\mathrm{Na}_{2} \mathrm{Te}+\mathrm{H}_{2} \mathrm{O} \stackrel{\mathrm{DMF}}{\rightarrow} \mathrm{NaHTe}+\mathrm{NaOH} \\
2 \mathrm{Te}+4 \mathrm{NaBH}_{4}+7 \mathrm{H}_{2} \mathrm{O} \rightarrow 2 \mathrm{NaHTe}+\mathrm{Na}_{2} \mathrm{~B}_{4} \mathrm{O}_{7}+14 \mathrm{H}_{2}
\end{gathered}
$$

As described in equation $6.1, \mathrm{Na}_{2} \mathrm{Te}$ can be prepared in DMF but if a trace of 
water present some NaHTe can form according to equation 6.2. This presence of side product $\mathrm{NaOH}$, as shown by the $\mathrm{pH}$ in variation in previous report (Table 6.1), would not affect the coarse of the reaction. Both $\mathrm{Na}_{2} \mathrm{Te}$ and $\mathrm{NaHTe}$ can react with tin precursor. These two compounds can be distinguished by colour, $\left(\mathrm{Na}_{2} \mathrm{Te}\right.$ : deep purple color; $\mathrm{NaHTe}$ pink or colourless).

While $\mathrm{H}_{2}$ Te gas can be prepared through either chemical decomposition of $\mathrm{Al}_{2} \mathrm{Te}_{3}$ powder according to reaction $6.4^{25}$ or electrochemical reduction of $\mathrm{Te}$ electrode in acid media according to equation $6.5 .^{23}$

$$
\begin{gathered}
\mathrm{Al}_{2} \mathrm{Te}_{3}+3 \mathrm{H}_{2} \mathrm{SO}_{4} \rightarrow \mathrm{Al}_{2}\left(\mathrm{SO}_{4}\right)_{3}+3 \mathrm{H}_{2} \mathrm{Te} \uparrow \\
\mathrm{Te} e^{0}+2 \mathrm{H}^{+}+2 e^{-} \rightarrow \mathrm{H}_{2} \mathrm{Te} \uparrow
\end{gathered}
$$

In addition, previous research has revealed that the optimized ratio of $\mathrm{Cd} / \mathrm{Te} / \mathrm{RSH}$ were $1: 0.5: 2.4$ or $1: 0.5: 1.3$ for using $\mathrm{NaHTe}$ or $\mathrm{H}_{2} \mathrm{Te}$ as the Te source, respectively (Table 6.1). The $\mathrm{pH}$ range for aqueous synthesis may affect the morphology of obtained products. Zhang and coworkers have demonstrated that telluride clusters tend to form at high $\mathrm{pH}$ values. ${ }^{26}$

Encouraged by the work in Chapter 5 and previous work in the literature, ${ }^{22,23}$ in this project, size-controlled water soluble SnTe nanoparticles will be synthesized in polar organic solvent and in the aqueous phase.

The main objective of this research was to synthesize water soluble SnTe nanoparticles of varying sizes using inexpensive and simple methods. The effects of reaction parameters on particle size and size distribution were investigated by TEM and XRD. More specifically, size distribution analysis was performed in the following experiments by measuring the sizes of more than 100 nanopar- 
ticles per system. The average crystallite size of SnTe was calculated from the line broadening of the strongest intensity (200) reflection, using the Scherrer equation and assuming spherical, defect-free crystals. Moreover, the crystal structure and crystallinity of the products were determined by SAED and XRD patterns. The chemical composition of the produced products were assessed by EDX analysis. The surface bonding of as-prepared nanoparticles was characterized by FTIR spectroscopy. The optical properties were measured by NIR spectroscopy using BOMEM DA8 spectrometer.

This chapter is divided into two main sections. Section 1 describes a series of experiments on the synthesis of SnTe nanoparticles using DMF as a solvent while section 2 delineates a series of experiments carried out in $\mathrm{H}_{2} \mathrm{O}$.

\subsection{Synthesis of SnTe nanoparticles in DMF}

In this series of experiments, SnTe nanoparticles were synthesized following a modification of the method adopted in the synthesis of SnS nanoparticles (see detail in Chapter 5). Water soluble SnS nanoparticles with a uniform particle size of $4 \mathrm{~nm}$ were prepared by reaction of $\mathrm{SnBr}_{2}$ with $\mathrm{Na}_{2} \mathrm{~S}$ at room temperature in the presence of triethanolamine (TEA) in ethylene glycol. Water soluble surfactant TEA has been demonstrated to afford better particle size control ${ }^{17}$ and thus this surfactant was chosen in the following experiments. $\mathrm{SnBr}_{2}$ was used as a $\mathrm{Sn}$ precursor and $\mathrm{NaHTe}$ was a Te source instead of $\mathrm{Na}_{2} \mathrm{~S}$ in the synthesis. Although $\mathrm{H}_{2}$ Te gas is another potential Te source, the preparation of fresh NaHTe solution is relatively easier than the generation of $\mathrm{H}_{2} \mathrm{Te}$ by using $\mathrm{Al}_{2} \mathrm{Te}_{3}$ or electrochemical methods. Moreover, N,N-dimethylformamide (DMF) was selected and used as solvent as it can provide better solubility for both Sn and Te precursors than EG. 
In addition, in the previous Chapter, it was found that further heating nanoparticles (particles prepared at a low temperature in advance) and using different amounts of TEA can significantly affect the particles size and size distribution. Hence, these effects were further investigated in the current SnTe system.

\subsubsection{Experiment A: Reaction at $50^{\circ} \mathrm{C}$}

Initially, SnTe nanoparticles were prepared through the reaction of $\mathrm{SnBr}_{2}$ with $\mathrm{NaHTe}$ at $50{ }^{\circ} \mathrm{C}$ in the presence of TEA in DMF. The ratio of $\mathrm{Sn} / \mathrm{Te}$ was set as 1: 0.5 . The reaction temperature was set at $50{ }^{\circ} \mathrm{C}$, as it is the lowest temperature that results in clear NaHTe solution in DMF. The reaction was performed under argon atmosphere.

\section{Experimental}

In a three neck round bottom flask, $0.1130 \mathrm{~g}(0.4 \mathrm{mmol}) \mathrm{SnBr}_{2}$ (anhydrous, Sigma Aldrich) was dissolved in a solution mixture of 2 mL TEA (LR, Pure Science) and $20 \mathrm{~mL}$ DMF (max $0.01 \% \mathrm{H}_{2} \mathrm{O}$, Scharlau), followed by heating to $50{ }^{\circ} \mathrm{C}$. After that, $2 \mathrm{~mL}$ of $0.1 \mathrm{M}$ freshly prepared NaHTe DMF solution was transferred into the $\mathrm{SnBr}_{2}$ solution with vigorous stirring. The fresh $\mathrm{NaHTe}$ solution was prepared by the reaction of $\mathrm{NaBH}_{4}$ with Te powder in a molar ratio of 1.3:1 in DMF. ${ }^{24}$ The colour of the solution changed from colorless to black immediately. The reaction mixture was held at $50{ }^{\circ} \mathrm{C}$ for $1 \mathrm{~min}$. The SnTe nanoparticles produced were separated out by centrifugation ( $12000 \mathrm{rpm}$ for 20 mins) and washed repeatedly in DMF for 3-4 times and stored in a suitable solvent for characterization. The obtained SnTe nanoparticles were characterized using TEM, EDX, SAED and XRD. 


\section{Results and discussion}

Fig. 6.1a shows typical HRTEM images of the products obtained at $50{ }^{\circ} \mathrm{C}$. The as-prepared SnTe nanoparticles have roughly spherical shape. A HRTEM image of an individual nanoparticle with atomic lattice fringes is clearly visible(inset, Fig. 6.1a).

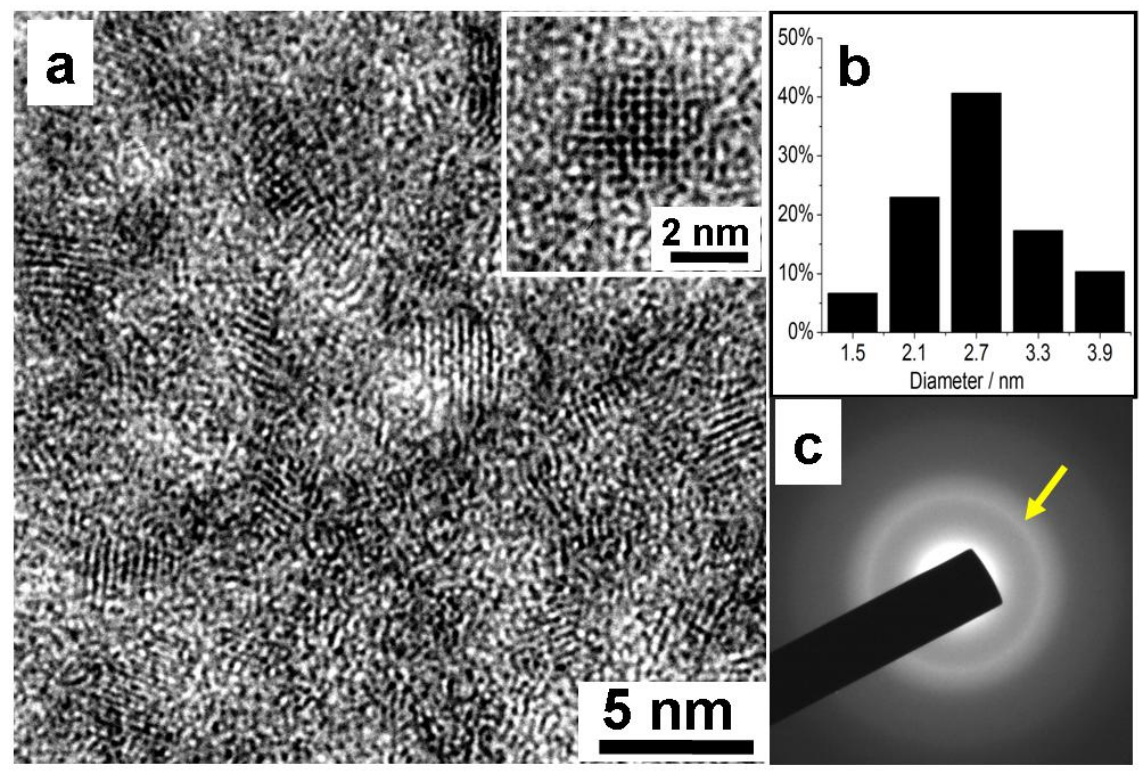

Fig. 6.1 (a)HRTEM image of SnTe nanoparticles synthesized at $50{ }^{\circ} \mathrm{C}$ (inset: HRTEM image of a single SnTe nanoparticle). (b) Histogram of nanoparticles size distribution, (c) Selected Area Electron Diffraction (SAED) pattern of cubic SnTe nanoparticles.

A histogram for nanoparticle size distribution taken from more than 100 nanoparticles is shown in Fig. $6.1 \mathrm{~b}$ which indicates particles of size $2.7 \pm 0.6 \mathrm{~nm}$. These results indicated that TEA is beneficial in controlling the particle growth. However, the SAED pattern (Fig. 6.1c) shows a broad diffuse ring which is due to the particles size being too small and close to the detection limit for SAED.

The powder X-ray Diffraction (XRD) pattern obtained from the same sample as above, Fig. 6.2 , shows only one broad peak at $\sim 32.8^{\circ}$. This is also due to the particles being too small and close to the detection limit for XRD. 


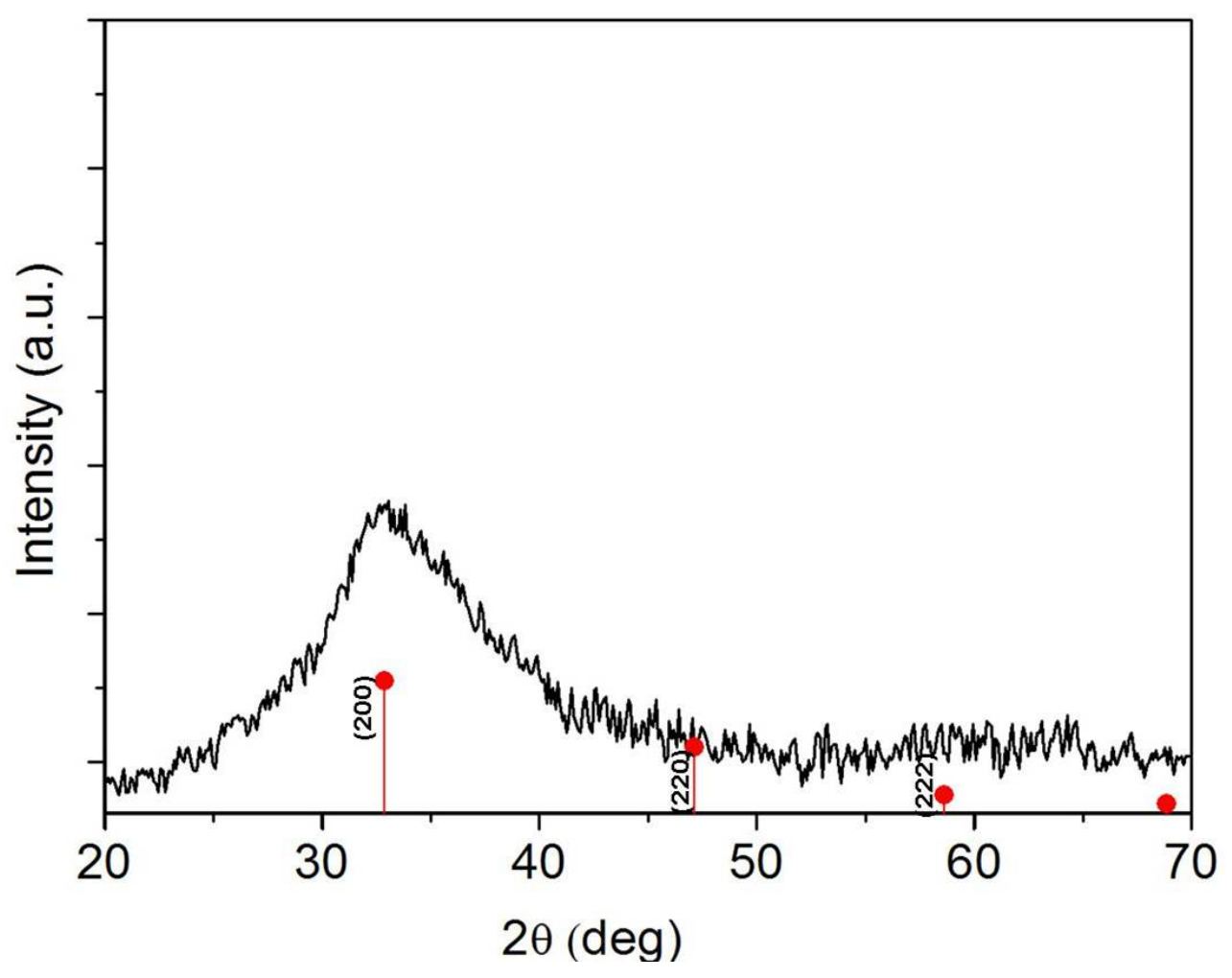

Fig. 6.2 XRD pattern of the SnTe nanoparticles obtained at $50{ }^{\circ} \mathrm{C}$ (Co radiation). The expected positions and intensities for peaks corresponding to cubic SnTe (ICDD 00-046-1210) are marked by lines labeled with "•".

The EDX spectrum of the as-synthesized particles, shown in Fig. 6.3, indicates that the atomic ratio of $\mathrm{Sn}$ to Te is $1.0: 0.96$, which is close to the stoichiometric composition SnTe.

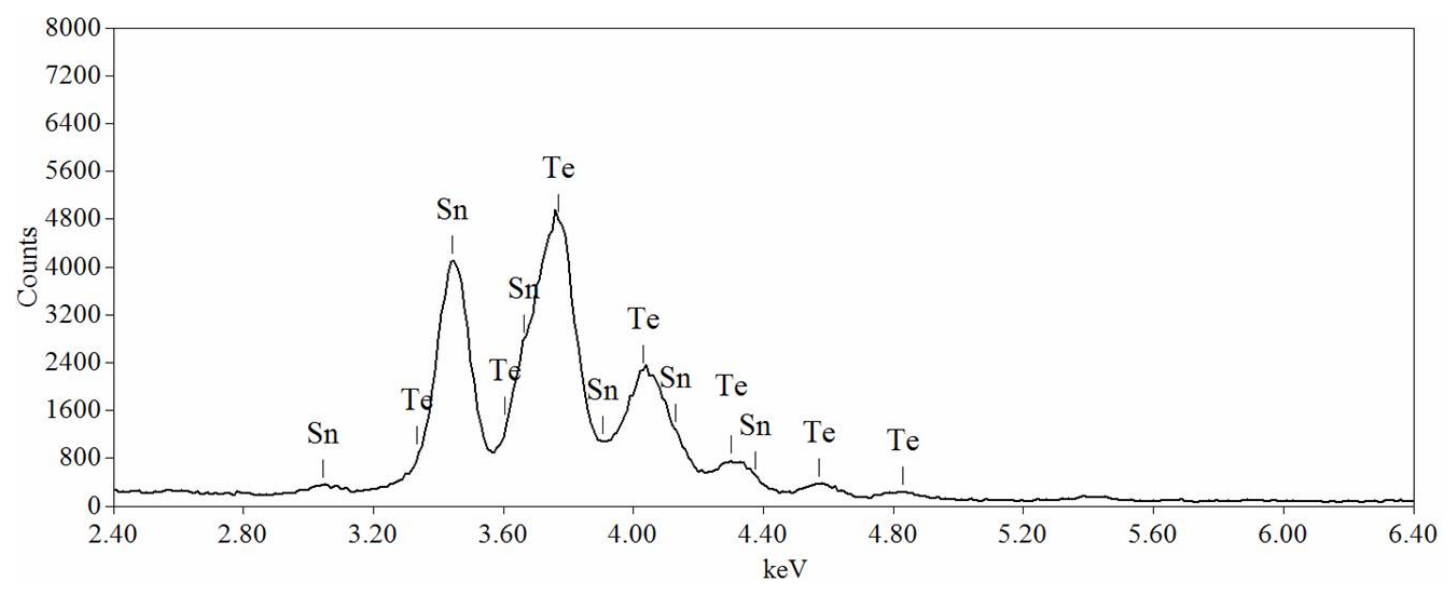

Fig. 6.3 EDX spectrum of the SnTe nanoparticles obtained at $50{ }^{\circ} \mathrm{C}$ in the presence of $2 \mathrm{~mL}$ TEA.

SnTe nanoparticles were prepared using $1: 0.5$ of $\mathrm{Sn} /$ Te precursors ratio which 
is similar to the ratio used in the literature for the synthesis of CdTe. ${ }^{23,26}$

A mechanism of SnTe nanoparticle formation is proposed and shown in Scheme 6.2. This formation mechanism is similar to that for SnS nanoparticles. ${ }^{17}$ TEA chelates with $\mathrm{Sn}^{2+}$ to form $\left[\mathrm{Sn}(\mathrm{TEA})_{n}\right]^{2+}$ intermediate and further react with $\mathrm{Te}^{2-}$ producing SnTe.

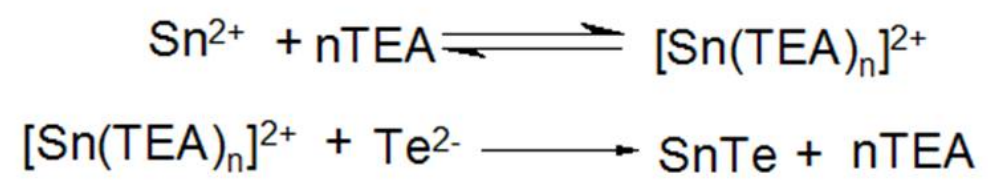

Scheme 6.2 Schematic illustration of the formation of SnTe nanoparticles.

After purification, the surface bonding of SnTe nanoparticles was characterized by FTIR spectroscopy (measured as KBr pellets) which showed the presence of triethanolamine capping ligand (Fig. 6.4).

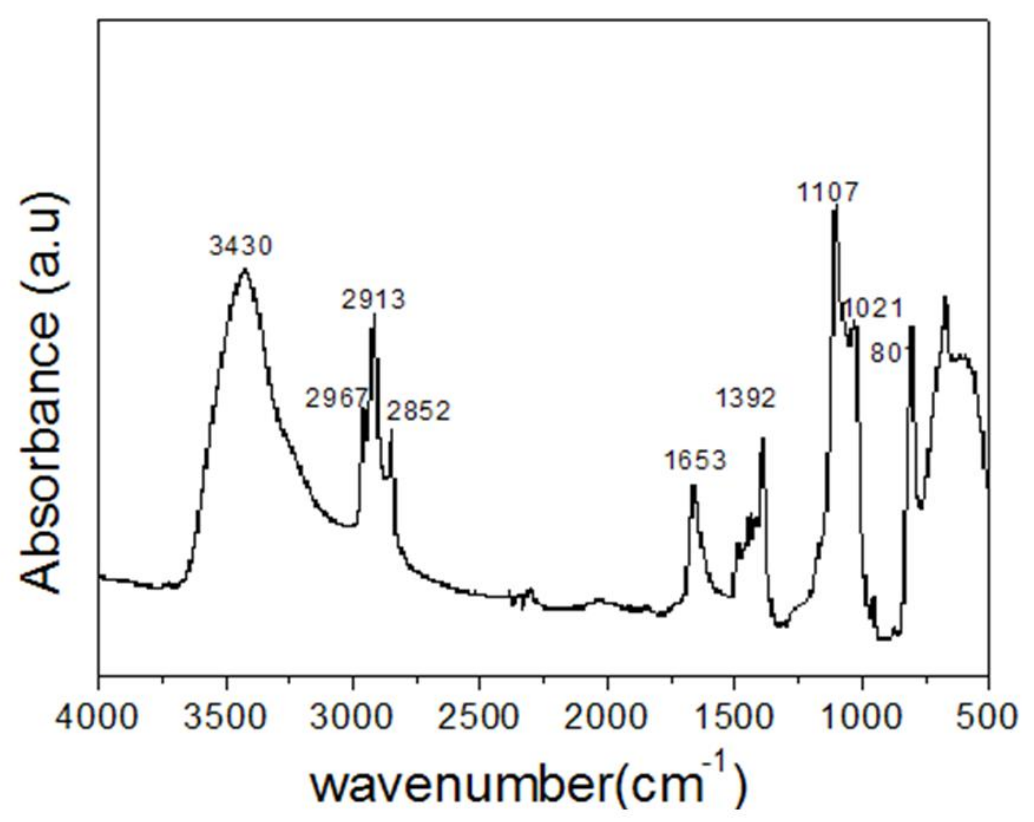

Fig. 6.4 FTIR spectra of TEA-capped SnTe nanoparticles. 
Band assignments show a significant absorbance in the region between 3500 $\mathrm{cm}^{-1}$ and $3000 \mathrm{~cm}^{-1}$ which is due to the strong $\mathrm{O}-\mathrm{H}$ stretching vibrations of the ligand and physisorbed and chemisorbed $\mathrm{H}_{2} \mathrm{O}$. The $2967 \mathrm{~cm}^{-1}, 2913 \mathrm{~cm}^{-1}$ and $2852 \mathrm{~cm}^{-1}$ bands correspond to the $\mathrm{C}-\mathrm{H}$ stretching vibrational mode. The $1653 \mathrm{~cm}^{-1}$ band is due to $\mathrm{H}-\mathrm{O}-\mathrm{H}$ bending of $\mathrm{H}_{2} \mathrm{O}$ molecules. The bands in the region of $1392 \mathrm{~cm}^{-1}$ is associated with vibrations of the C-C and C-H bonds. The C-O and C-N stretching vibration were detected at $1107^{-1}, 1021 \mathrm{~cm}^{-1}$, respectively. The band at $801 \mathrm{~cm}^{-1}$ could be due to C-N, C-C, C-O and C-C-O vibrations.

In summary, this initial experiment has provided evidence that this approach has some promise. Water soluble SnTe nanoparticles can be prepared through the synthetic approach. The resulting SnTe nanoparticles have spherical shape, fine size $(\sim 2 \mathrm{~nm})$ and narrow size distribution.

\subsubsection{Experiment B-C: Effect of reflux time}

To prepare highly crystalline SnTe nanoparticles, a thermal treatment is often necessary. ${ }^{27}$ In the present research, reflux was employed (DMF boiling point: $\left.150{ }^{\circ} \mathrm{C}\right) \cdot{ }^{28}$

In the following experiment B and C, SnTe nanoparticles were prepared under reflux for 2 and 5 minutes. More specifically, the SnTe nanoparticles were first prepared at $50{ }^{\circ} \mathrm{C}$ for $1 \mathrm{~min}$ (same as experiment $\mathrm{A}$ ) and then further refluxed for 2 minutes (experiment B) and 5 minutes (experiment $C$ ).

\section{Results: Experiment B (refluxing for $2 \mathrm{~min}$ )}

Fig. 6.5a shows a typical TEM image of SnTe nanoparticles obtained by refluxing for 2 minutes. The particles have a nearly spherical shape with an average 
size of $6.5 \pm 1.2 \mathrm{~nm}$ (Fig. 6.5b).

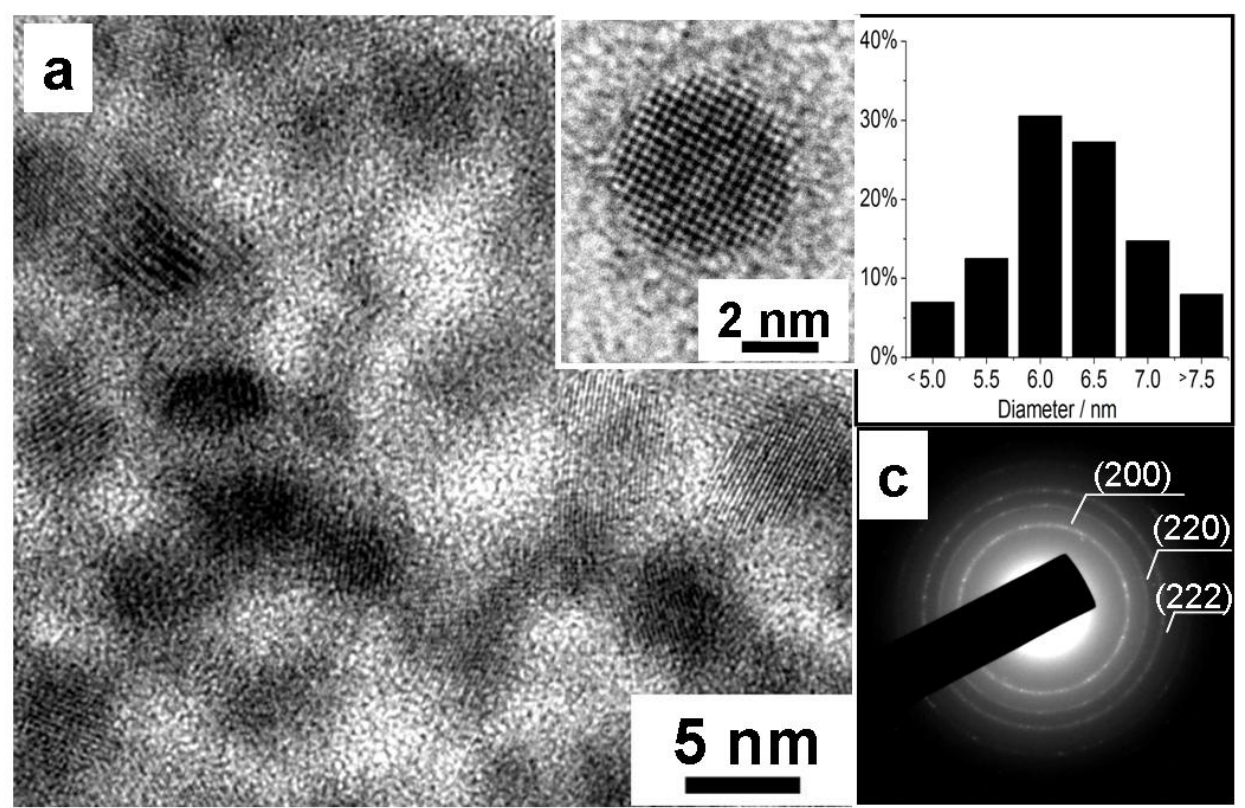

Fig. 6.5 (a)TEM image of SnTe nanoparticles synthesized at $50^{\circ} \mathrm{C}$, reflux for 2 minutes (inset: HRTEM image for a single SnTe nanoparticle). (b) Histogram of nanoparticles size distribution, (c) SAED pattern of cubic SnTe nanoparticles.

A HRTEM image (Inset, Fig. 6.5a) of an individual nanoparticle with atomic lattice fringes clearly visible, demonstrates the cubic crystal structure of the SnTe nanoparticles. The sharp diffraction rings in SAED pattern (Fig. 6.5c) can be indexed to the cubic phase of SnTe.

Fig. 6.6 shows the XRD pattern of the as-prepared SnTe nanoparticles. All of the index peaks in the spectrum match well with that of the bulk material, in a face-centered cubic structure. No peaks due to elemental tin or tellurium were observed in the pattern. The crystallite size of the SnTe nanoparticles estimated from the broadening of the XRD reflections peak was $6.5 \pm 0.7 \mathrm{~nm}$. An EDX spectrum pattern, as shown in Fig. 6.7, revealed that the nanoparticles were composed of $50 \%$ tin and $50 \%$ tellurium. 


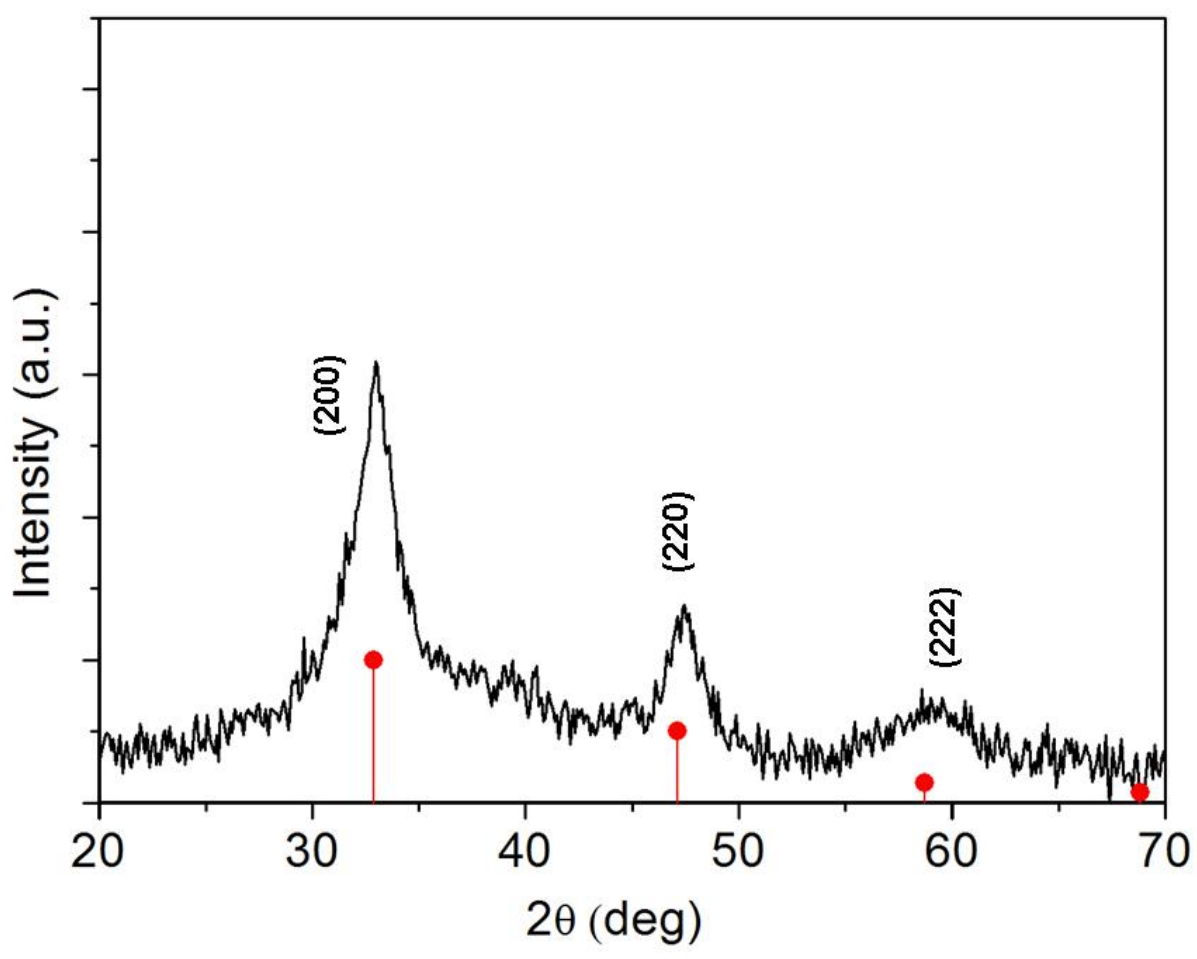

Fig. 6.6 XRD pattern of as-prepared SnTe nanoparticles (Co radiation). The expected positions and intensities for peaks corresponding to cubic SnTe (ICDD 00-046-1210) are marked by lines labeled with "•".

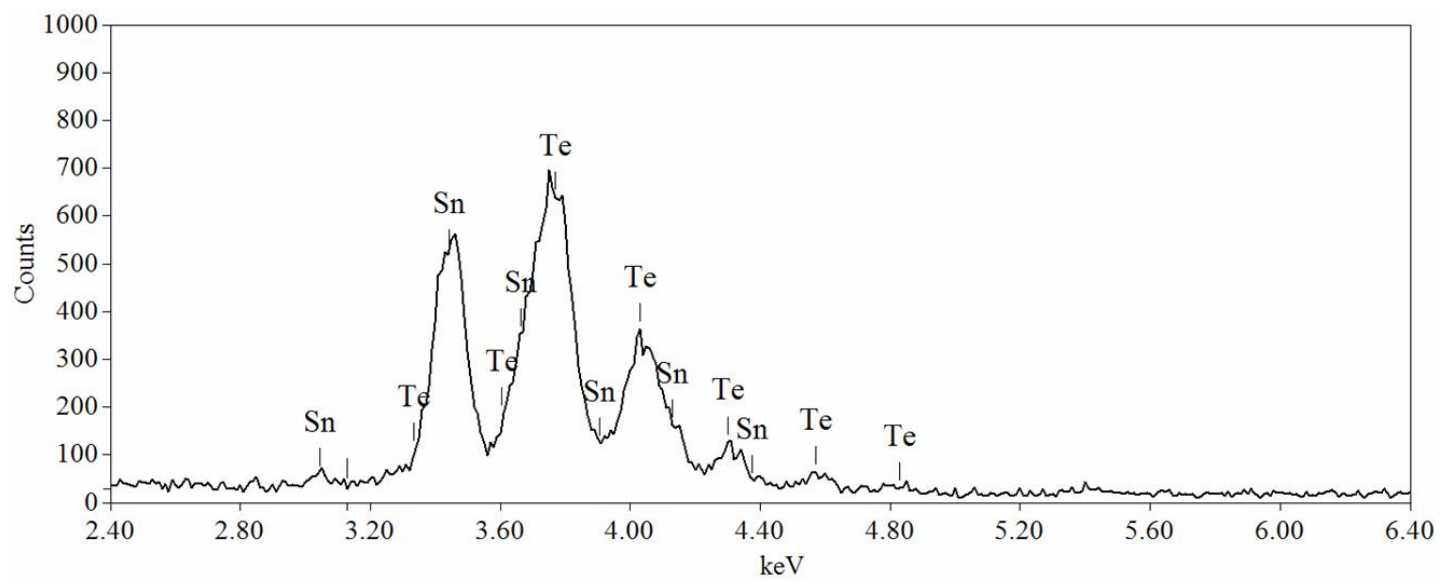

Fig. 6.7 EDX spectrum of as-prepared SnTe nanoparticles.

\section{Results: Experiment C (refluxing for 5 min)}

The LRTEM image of the SnTe nanoparticles refluxed for 5 minutes is shown in Fig. 6.8. The nanoparticles are $15.5 \pm 1.5 \mathrm{~nm}$ in size and have roughly spher- 
ical shape and tend to aggregate. The sharp rings in SAED pattern (Fig. 6.8b) shows the cubic crystalline nature.

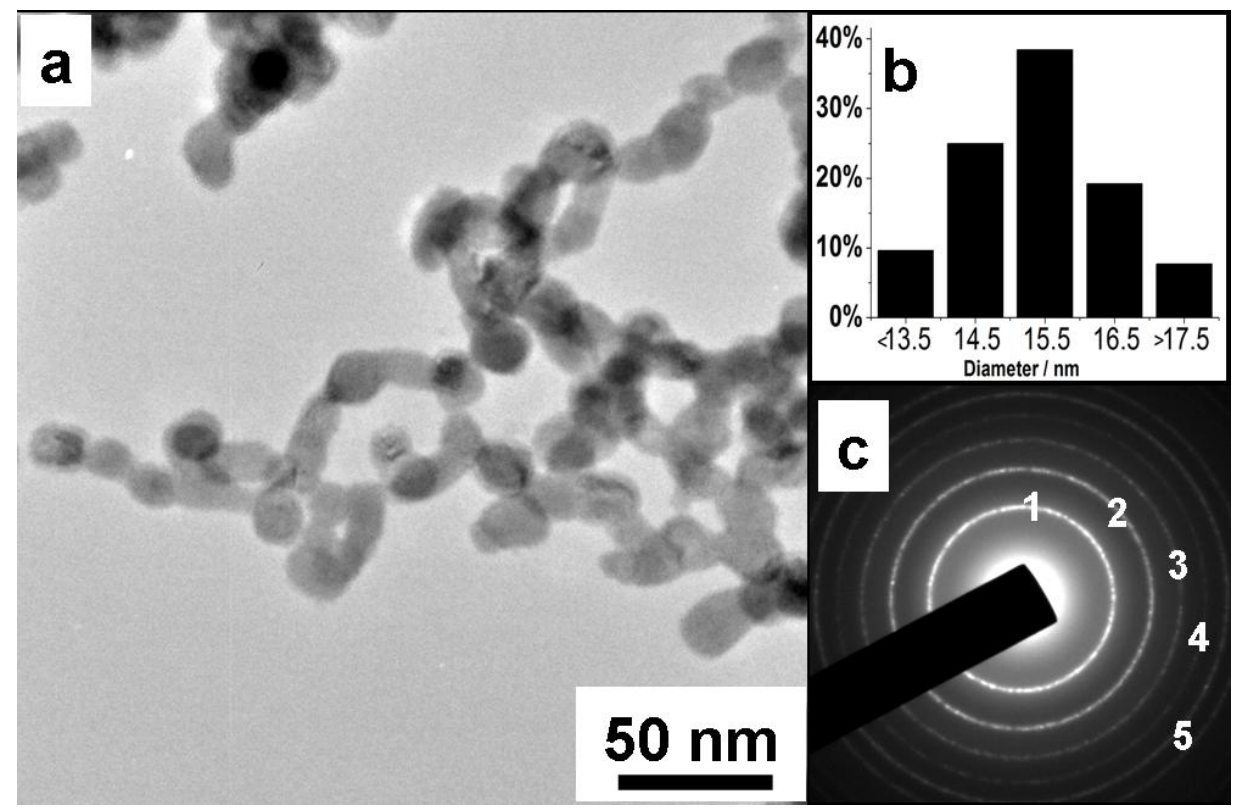

Fig. 6.8 (a) A typical TEM image of SnTe nanoparticles synthesized at $50{ }^{\circ} \mathrm{C}$, refluxed for 5 minutes. (b) Histogram of nanoparticle size distribution (c) SAED pattern of the SnTe nanoparticles. 1-(200); 2-(220); 3-(222); 4-(400); 5(420).

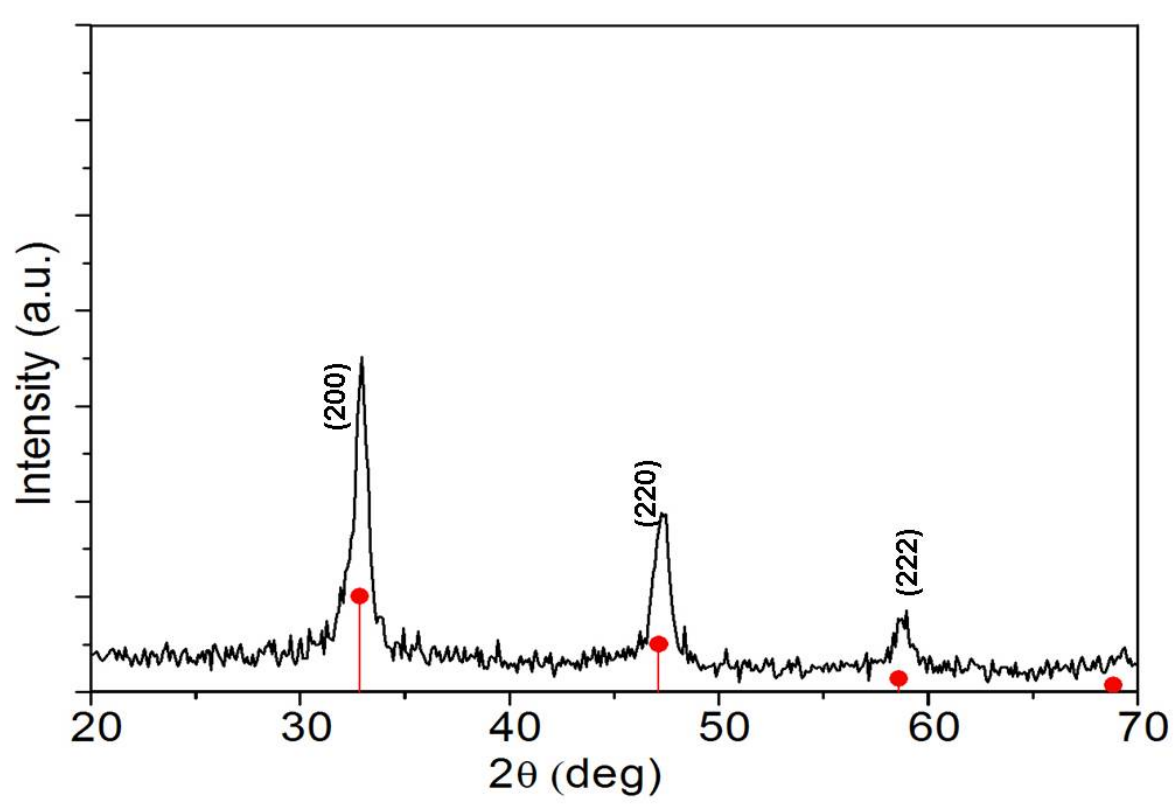

Fig. 6.9 XRD pattern of the SnTe nanoparticles obtained at $50{ }^{\circ} \mathrm{C}$,reflux for $5 \mathrm{~min}$ (Co radiation). The expected positions and intensities for peaks corresponding to cubic SnTe (ICDD 00-046-1210) are marked by lines labeled with $" \bullet "$. 
The crystalline phase and crystallite size of the as-prepared product were further confirmed and identified from the measured powder XRD pattern(Fig. 6.9). The sharp and intense diffraction peaks can be indexed to the cubic structure of SnTe. The calculated crystallite size is $10.2 \pm 1.0 \mathrm{~nm}$. EDX analysis confirms the presence of $51 \%$ Sn and $49 \%$ Te(Fig. 6.10).

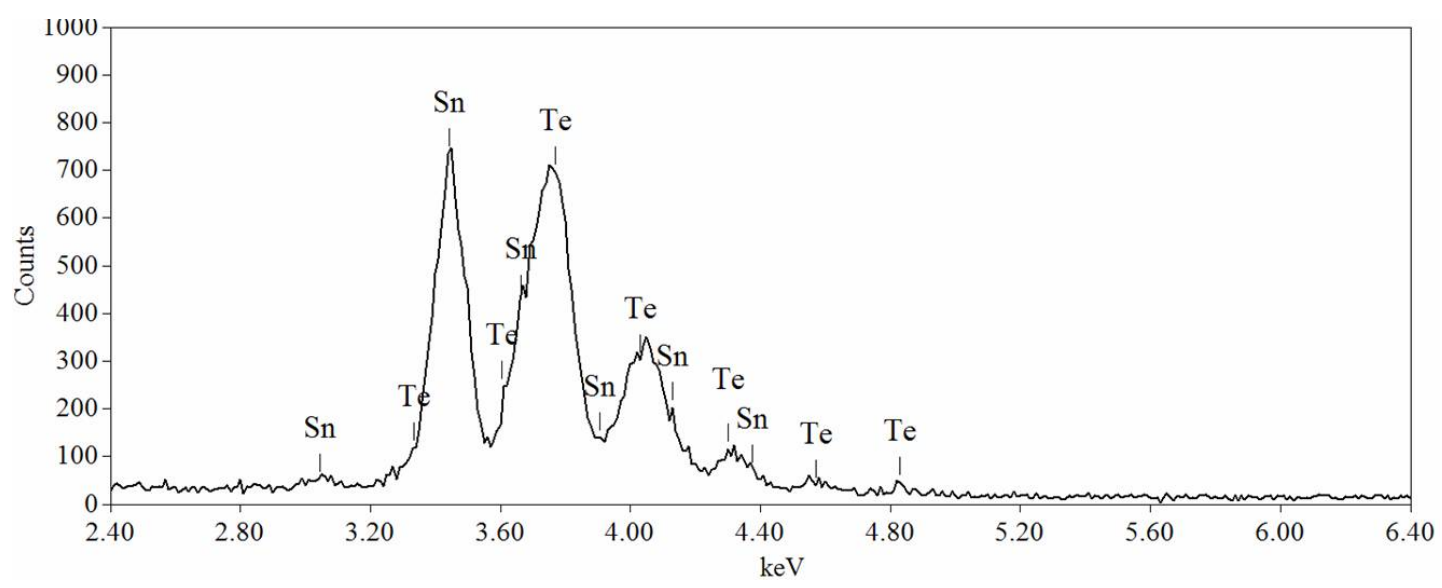

Fig. 6.10 EDX spectrum of the SnTe nanoparticles obtained at $50{ }^{\circ} \mathrm{C}$, reflux for $5 \mathrm{~min}$

\section{Discussion: Effect of reflux time}

Table 6.2 summarizes the results of SnTe nanoparticles obtained at different reflux time. It was found that the as-prepared SnTe nanoparticles were mainly of roughly spherical shape which is thermodynamically stable. ${ }^{29}$ Enhanced crystallinity (cubic structure) was achieved after heat treatment, which is consistent with the literature. It is known that higher temperature treatment forms better crystalline nanoparticles. ${ }^{27}$ The observed growth in particle size with increasing reflux time (from $2.7 \mathrm{~nm}$ without reflux to $15.5 \mathrm{~nm}$ after 5 minutes reflux) is due to the Ostwald ripening, smaller particles continue to shrink while larger particles grow. ${ }^{29}$ In addition to the increase in size, the nanoparticles appeared agglomerated after 5 minutes reflux in solution to form a precipitate. This may be due to the decreased repulsion force between the particles by heating leading to larger irregular aggregates. The average crystallite sizes obtained by 
XRD are almost equal to the average particle sizes obtained by TEM indicating that most of SnTe nanoparticles are single crystals.

Table 6.2 Summary of results for SnTe nanoparticles obtained with varying reflux time.

\begin{tabular}{|c|c|c|c|c|c|}
\hline & Reflux & & Product & & \\
\hline $\operatorname{Exp}$ & $(\min )$ & Morphology & Crystal structure & Particle size & Crystallite size \\
\hline A & 0 & $\begin{array}{l}\text { roughly spheri- } \\
\text { cal NPs }\end{array}$ & crystalline & $2.7 \pm 0.6 \mathrm{~nm}$ & \\
\hline B & 2 & $\begin{array}{l}\text { roughly spheri- } \\
\text { cal NPs }\end{array}$ & $\begin{array}{l}\text { crystalline, cubic } \\
\text { phase }\end{array}$ & $6.5 \pm 1.2 \mathrm{~nm}$ & $6.5 \pm 0.7 \mathrm{~nm}$ \\
\hline $\mathrm{C}$ & 5 & $\begin{array}{l}\text { roughly spheri- } \\
\text { cal NPs }\end{array}$ & $\begin{array}{l}\text { crystalline, cubic } \\
\text { phase }\end{array}$ & $\begin{array}{l}15.5 \pm 1.5 \\
\mathrm{~nm}\end{array}$ & $10.2 \pm$ \\
\hline
\end{tabular}

In summary, the reaction at $50{ }^{\circ} \mathrm{C}$ with further reflux for 2 minutes is the optimal reaction condition to produce highly crystalline SnTe nanoparticles with particle size of $\sim 6.5 \mathrm{~nm}$. Without reflux, the product was poorly crystalline but with longer reflux time (5 minutes), there was a tendency to produce large aggregates.

\subsubsection{Experiment D-G: Effect of the amount of TEA ligand}

To probe the effect of the amount of TEA ligand on the synthesis and optical properties, four additional reactions were performed at different amounts of TEA ligand including $0.5 \mathrm{~mL}, 1 \mathrm{~mL}, 4 \mathrm{~mL}$ and $6 \mathrm{~mL}$ TEA, maintaining other experimental conditions same as in experiment B.

\section{Results: Experiment D (0.5 mL TEA)}

Fig. 6.11a shows a typical low magnification TEM image of the SnTe nanoparticles grown in the presence of $0.5 \mathrm{~mL}$ TEA. The particles have irregular shape morphologies and a large size distribution $(10-60 \mathrm{~nm})$. The average size of the SnTe nanoparticles is $32.4 \pm 11.5 \mathrm{~nm}$ (Fig. 6.11b). The spotty nature of the SAED pattern (Fig. 6.11c) can be indexed to the cubic phase of SnTe. 


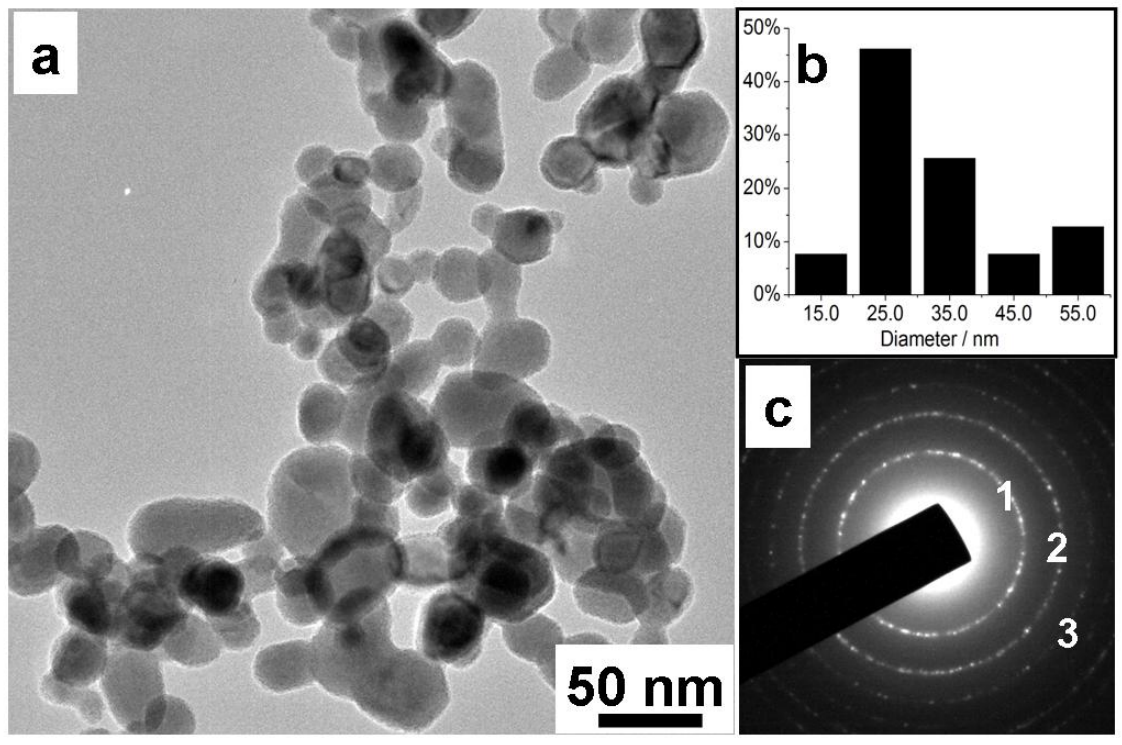

Fig. 6.11 (a) Typical low magnification TEM image of SnTe nanoparticles synthesized using $0.5 \mathrm{~mL}$ TEA as surfactant.(b) Histogram of nanoparticles size distribution, (C) SAED pattern of obtained nanoparticles. 1-(200); 2-(220); 3(222).

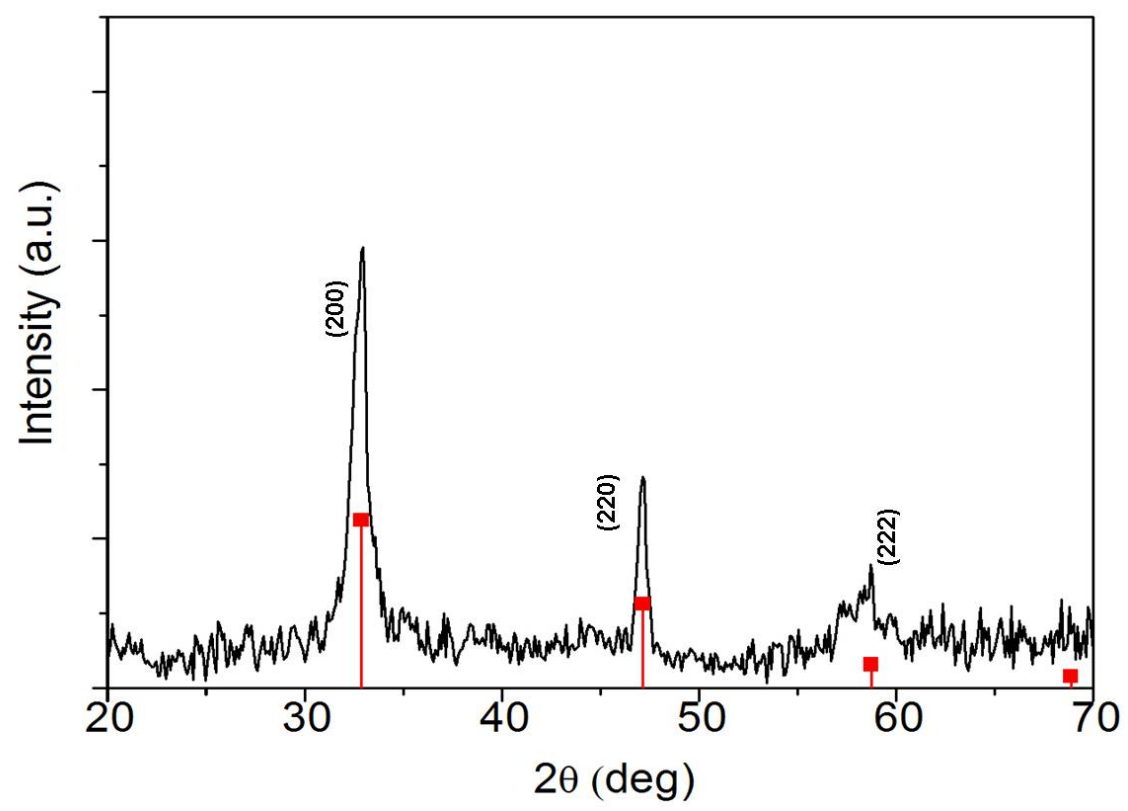

Fig. 6.12 XRD pattern of as-prepared SnTe nanoparticles (Co radiation). The expected positions and intensities for peaks corresponding to cubic SnTe (ICDD 00-046-1210) are marked by lines labeled with "•".

SAED (Fig. 6.11c) and XRD (Fig. 6.12) studies confirmed the formation of a cubic phase SnTe without detectable impurities. The average crystallite size of the nanoparticles, estimated from XRD data using the Scherrer's equation, 
was $17.1 \pm 2.0 \mathrm{~nm}$. An EDX spectrum,as shown in Fig. 6.13, revealed that the nanoparticles were composed of $51 \%$ tin and $49 \%$ tellurium.

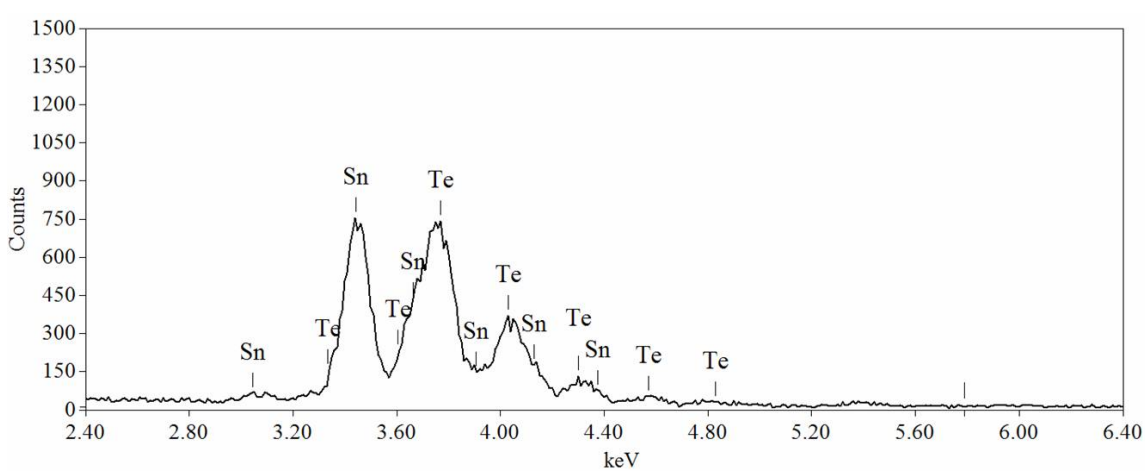

Fig. 6.13 EDX spectrum of the SnTe nanoparticles obtained using 0.5 mL TEA as a surfactant

\section{Results: Experiment E (1.0 mL TEA)}

A typical low magnification TEM image of the as-prepared nanoparticles is shown in Fig. 6.14a. Most of the nanoparticles have a roughly spherical shape while some particles appear irregular in shape. The as-prepared particles have an average size of $17.9 \pm 3.4 \mathrm{~nm}$ (Fig. 6.14b).

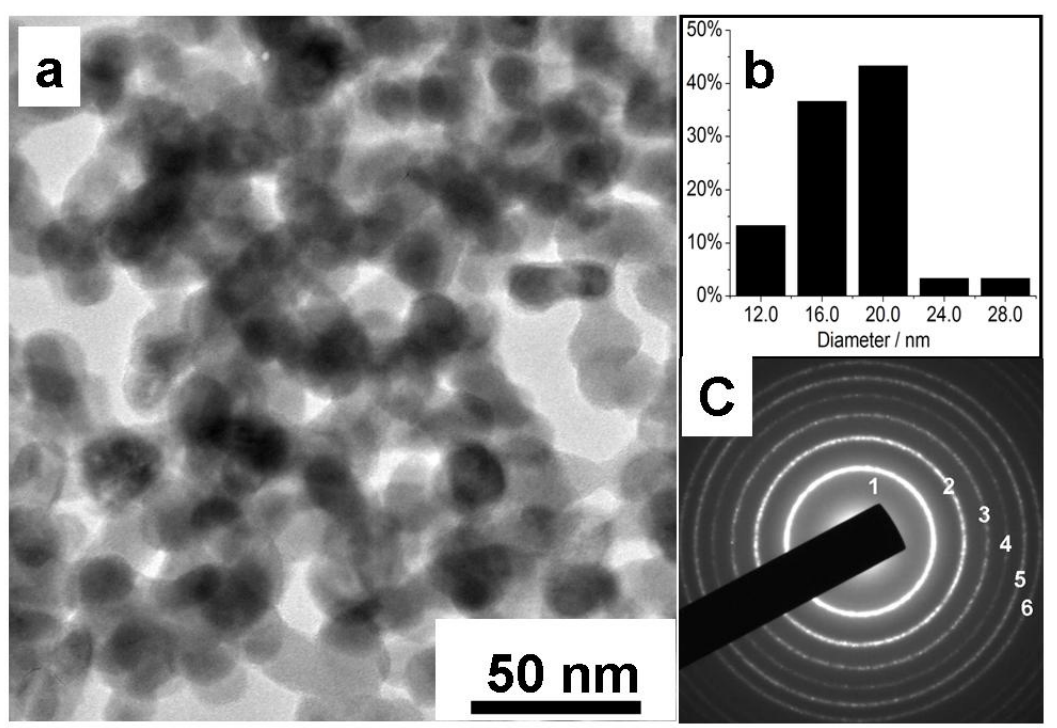

Fig. 6.14 (a) Typical TEM image of SnTe nanoparticles synthesized using 1.0 mL TEA as surfactant. (b) Histogram of nanoparticles size distribution, (c) SAED pattern of the SnTe nanoparticles.1-(200); 2-(220); 3-(222); 4-(400); 5-(420) and 6-(422). 
The selected area electron diffraction (SAED) pattern, Fig. 6.14c, has a strong and sharp set of diffraction rings. These diffraction rings correspond to the planes of cubic rock salt structure SnTe (space group Fm3m, a = 6.328 $\AA$ ).

X-ray analysis (Fig. 6.15) showed no impurities, and the lattice parameters are consistent with the SAED analysis. The average crystallite size was estimated from the XRD pattern of $9.4 \pm 1.0 \mathrm{~nm}$. EDX elemental analysis (Fig. 6.16) confirmed that the as-prepared nanoparticles contained $50 \%$ tin and 50\% tellurium.

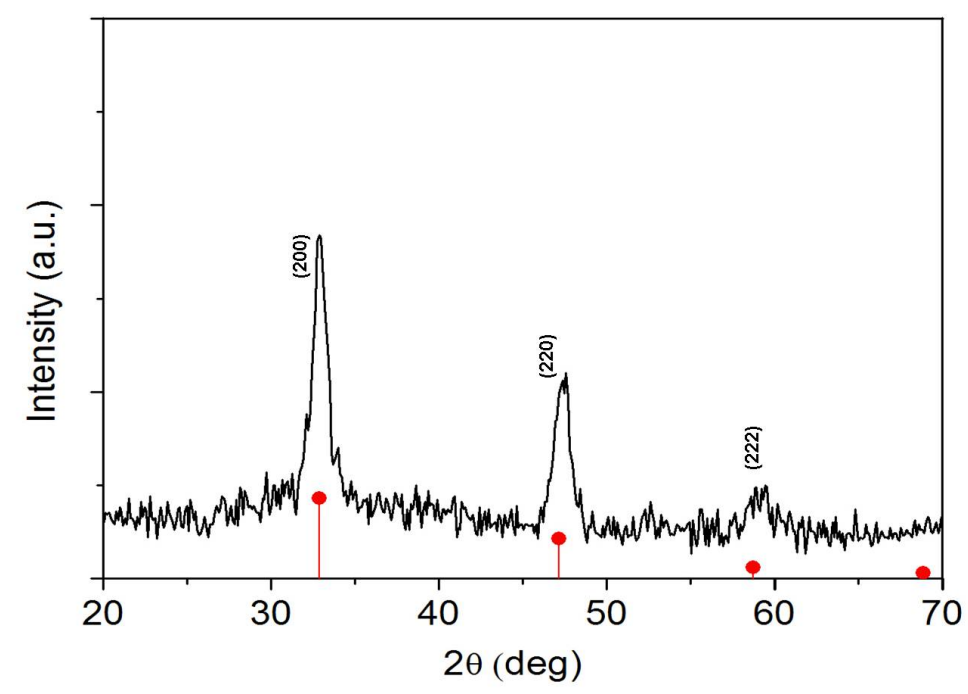

Fig. 6.15 XRD pattern of as-prepared SnTe nanoparticles (Co radiation). The expected positions and intensities for peaks corresponding to cubic SnTe (ICDD 00-046-1210) are marked by lines labeled with "•".

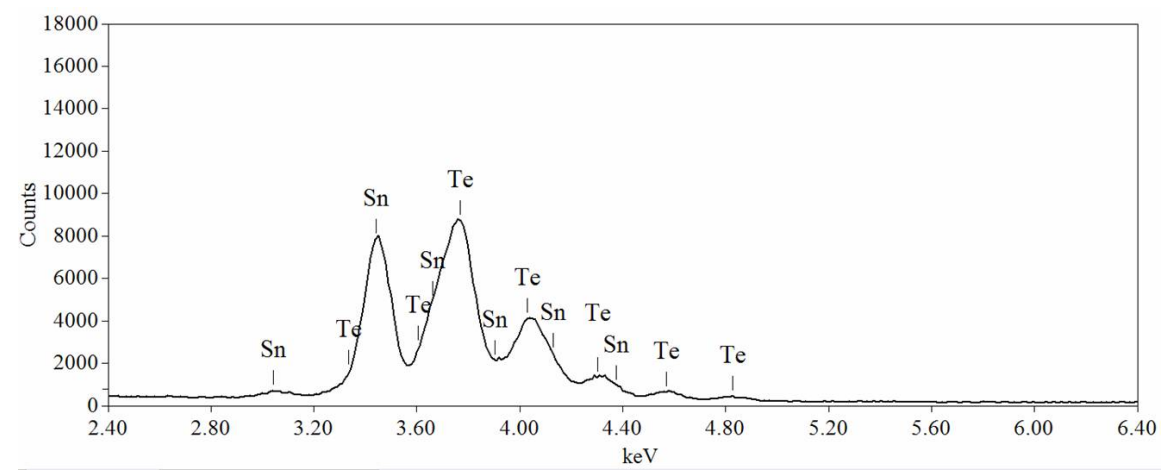

Fig. 6.16 EDX spectrum of the SnTe nanoparticles obtained using $1 \mathrm{~mL}$ TEA as a surfactant. 


\section{Results: Experiment F (4.0 mL TEA)}

A typical low magnification TEM image of the product obtained in the presence of 4 mL TEA, Fig. 6.17a, show that the particles appear to have irregular spherical shape, with an average size about $12.5 \pm 2.0 \mathrm{~nm}$.

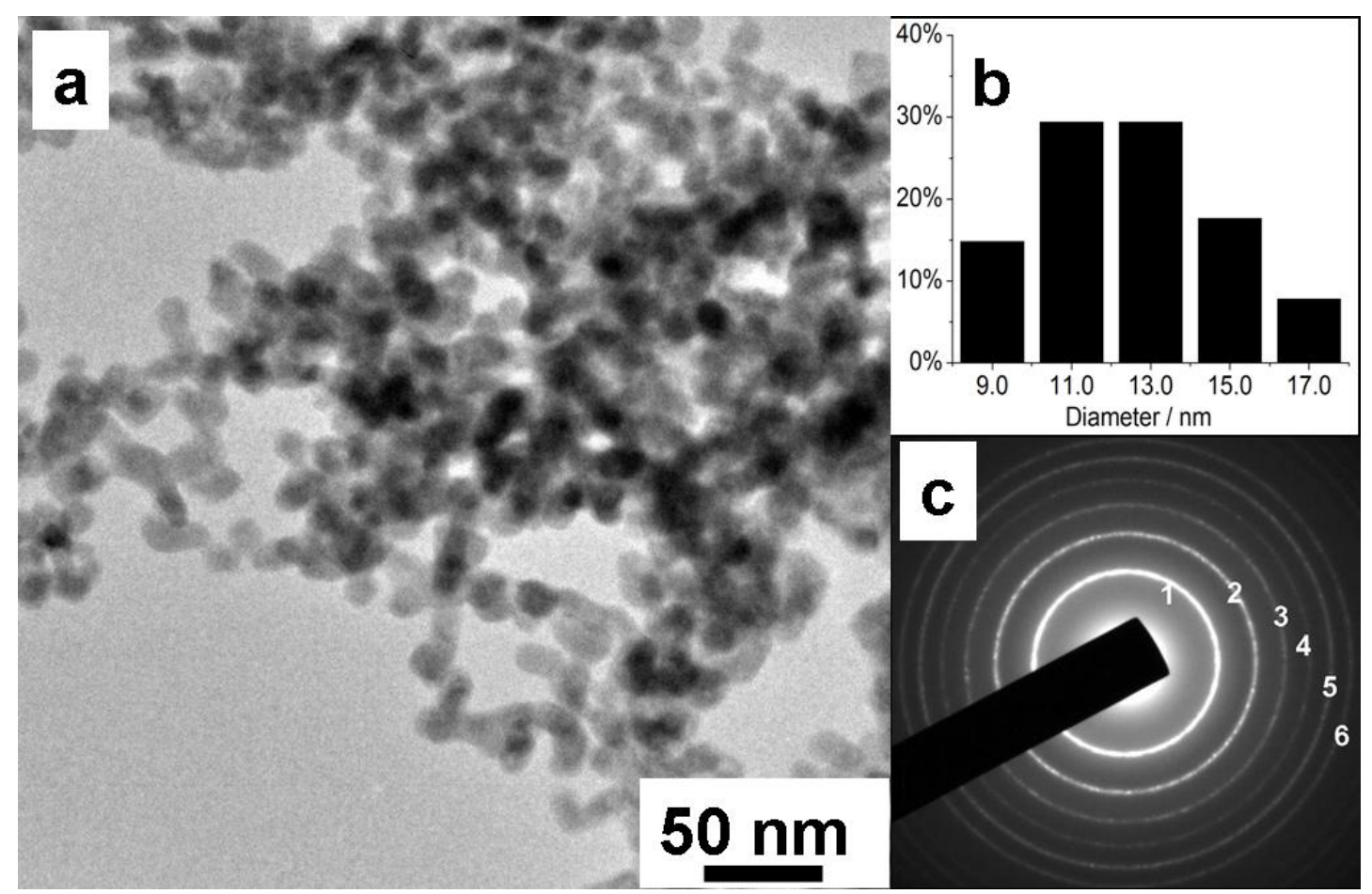

Fig. 6.17 (a) Typical low magnification TEM image of SnTe nanoparticles synthesized using $4.0 \mathrm{~mL}$ TEA as surfactant.(b)Histogram of nanoparticles size distribution.(c) SAED pattern of the SnTe nanoparticles. 1-(200); 2-(220); 3(222); 4-(400); 5-(420) and 6-(422).

SAED (Fig. 6.17b) and XRD (Fig. 6.18) pattern analysis confirmed that the obtained nanoparticles were cubic phase SnTe. The average crystallite size calculated from XRD was $12.3 \pm 1.0 \mathrm{~nm}$. EDX analysis (Fig. 6.19) showed that the nanoparticles contained $51 \%$ tin and $49 \%$ tellurium. 


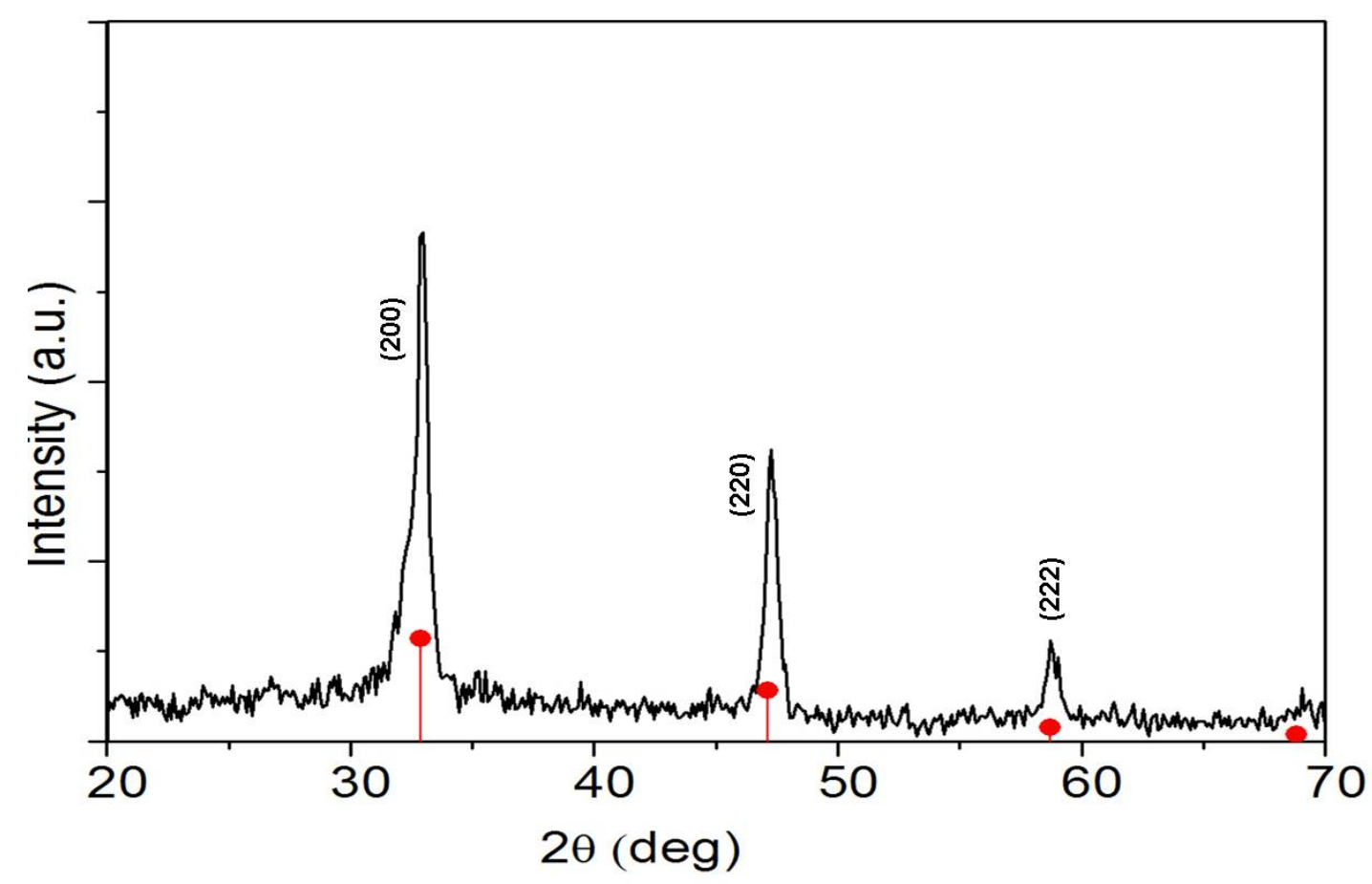

Fig. 6.18 XRD pattern of the SnTe nanoparticles obtained using $4 \mathrm{~mL}$ TEA as surfactant (Co radiation). The expected positions and intensities for peaks corresponding to cubic SnTe (ICDD 00-046-1210) are marked by lines labeled with "•".

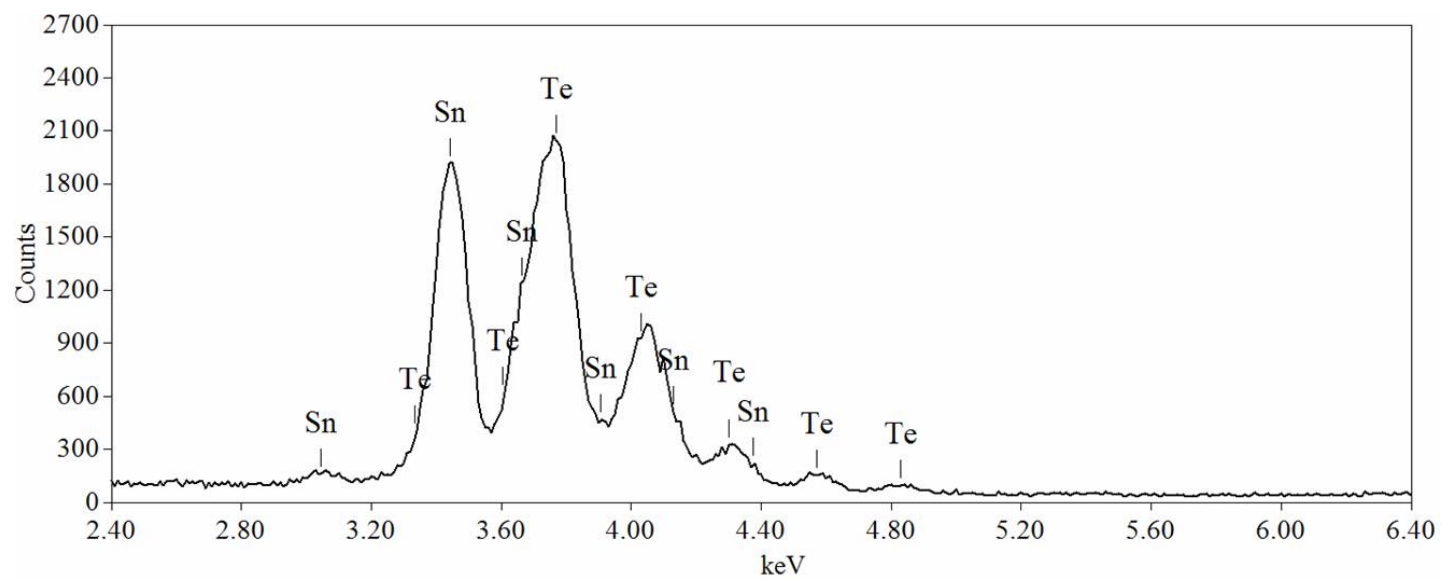

Fig. 6.19 EDX spectrum of as-prepared SnTe nanoparticles.

\section{Results: Experiment G (6.0 mL TEA)}

Fig. 6.20a shows a typical TEM image of the product obtained in the presence of $6 \mathrm{~mL}$ TEA. The particles possess roughly spherical shape, with an average size about $13.5 \pm 2.0 \mathrm{~nm}$. 


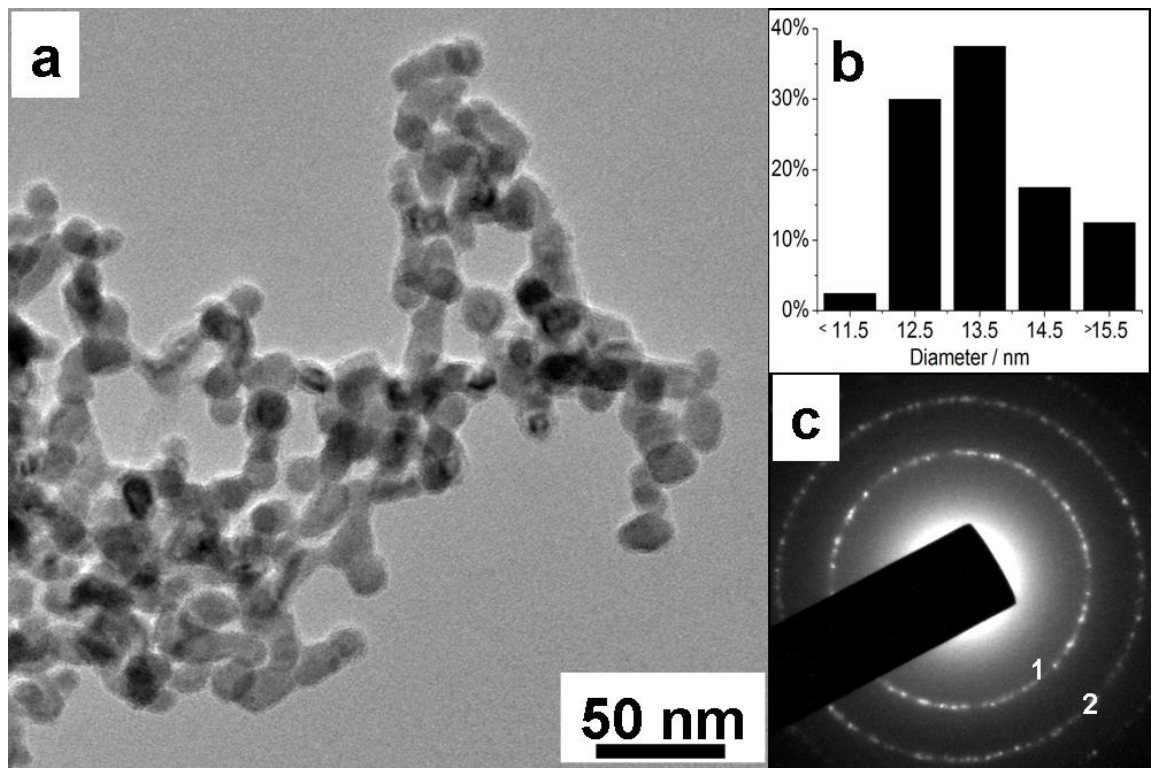

Fig. 6.20 (a) Typical low magnification TEM image of SnTe nanoparticles synthesized using $6.0 \mathrm{~mL}$ TEA as surfactant.(b)Histogram of nanoparticles size distribution. (c) SAED pattern of the SnTe nanoparticles. 1-(200); 2-(220).

SAED (Fig. 6.20b) and XRD (Fig. 6.21) pattern analysis both confirmed that the obtained nanoparticles were cubic phase SnTe. The average crystallite size calculated from XRD data was $9.7 \pm 1.0 \mathrm{~nm}$. EDX analysis (Fig. 6.22) showed that the nanoparticles contained approximately 53 atom\% tin and 47 atom\% tellurium.

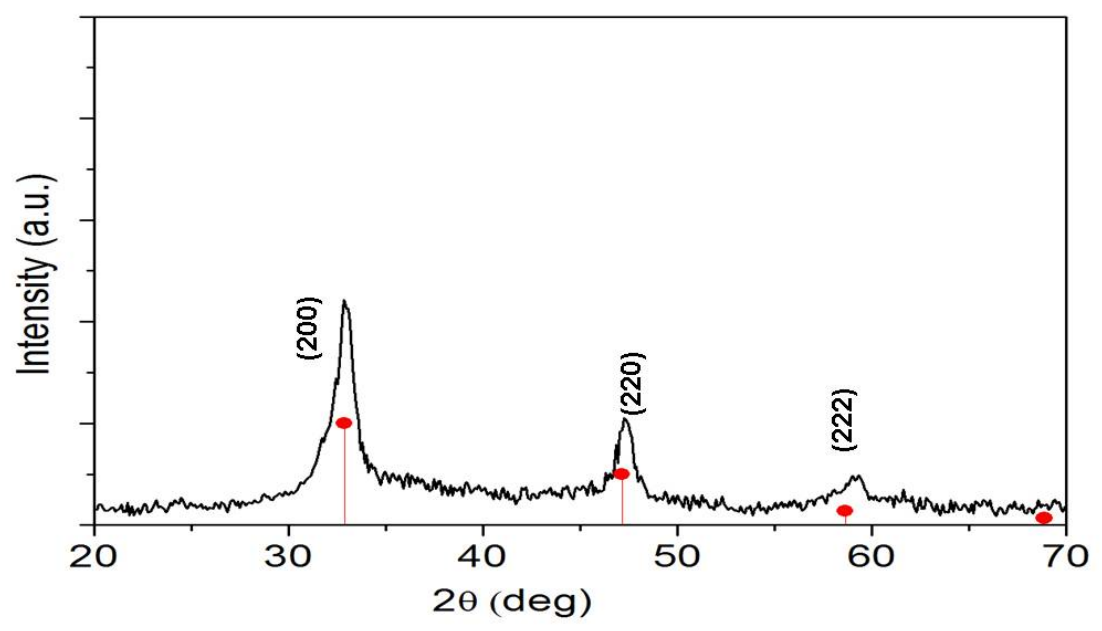

Fig. 6.21 XRD pattern of the SnTe nanoparticles obtained using $6 \mathrm{~mL}$ TEA as surfactant (Co radiation). The expected positions and intensities for peaks corresponding to cubic SnTe (ICDD 00-046-1210) are marked by lines labeled with" "." 


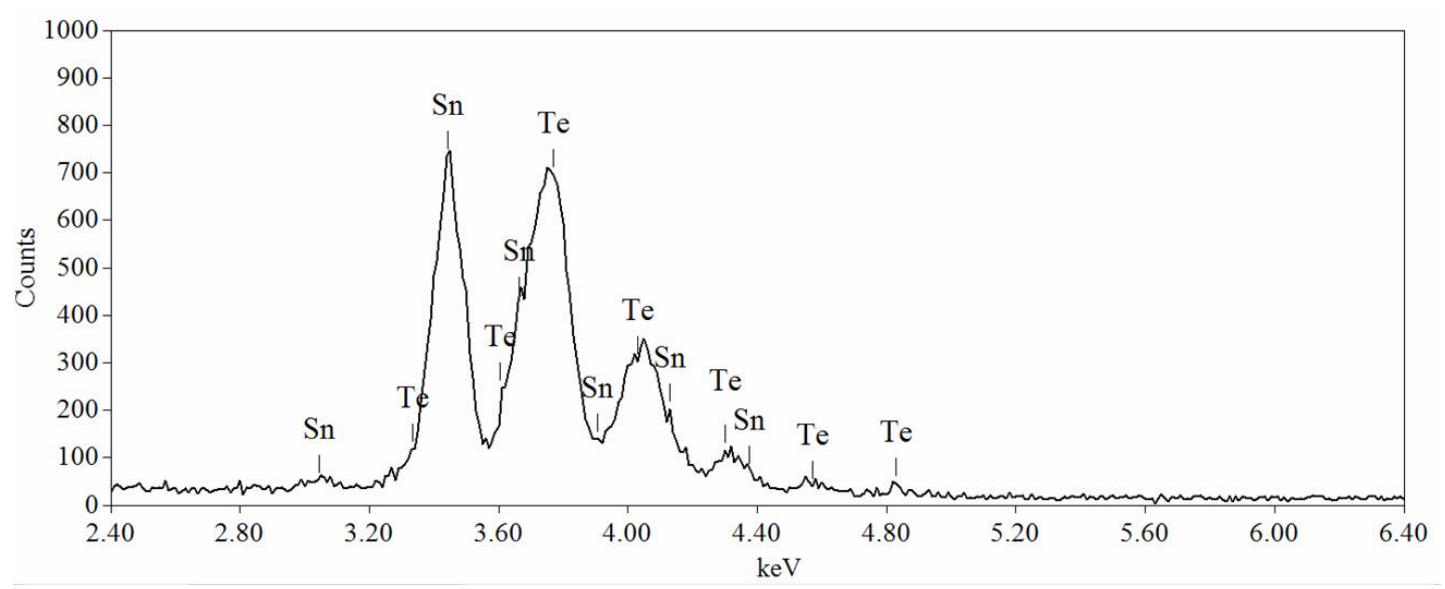

Fig. 6.22 EDX spectrum of as-prepared SnTe nanoparticles.

\section{Discussion: Effect of the amount of TEA ligand}

The results summarized in Table 6.3 show that the size of SnTe nanoparticles varied with the amount of TEA ligand in reaction system. Using less amount of TEA (0.5 mL and $1 \mathrm{~mL})$ led to formation of larger size SnTe nanoparticles with broad size distribution. This suggests that the amount of TEA used was insufficient to stabilize the SnTe nanoparticles. A similar observation was observed in the synthesis of SnS nanoparticles (see Chapter 5). When $2 \mathrm{~mL}$ TEA ligand was used in the reaction, the as-prepared SnTe nanoparticles exhibited narrow size distribution with small average size. This can be explained by the stronger stabilizing effect achieved with greater amount of surfactant. However, when the amount of TEA used was above $2 \mathrm{~mL}$, the average nanoparticle size was increased again with a bigger size distribution. This can be explained by the formation of an intermediate chelate complex $\left[\operatorname{Sn}(\mathrm{TEA})_{n}\right]^{2+}$ in the solution. ${ }^{17}$ This complex could be more stable when a larger amount of TEA is present in solution. Therefore, when the Te source was added into the $\mathrm{SnBr}_{2}$ solution, a smaller number of nuclei formed in the presence of the large amount of TEA (4 $\mathrm{mL}$ or $6 \mathrm{~mL}$ ). These nuclei would grow to larger size particles by reflux. Based on the experimental results obtained above, the optimal amount of TEA used in the reaction was $2 \mathrm{~mL}$ to form monodispersed SnTe nanoparticles with small 
particle size $(\sim 6.5 \mathrm{~nm})$.

Table 6.3 Summary of results for SnTe nanoparticles obtained with varying the amount of TEA in DMF

\begin{tabular}{|c|c|l|l|l|l|}
\hline \multirow{2}{*}{ Exp } & \multirow{2}{*}{ TEA } & \multicolumn{4}{|c|}{ Products } \\
\cline { 3 - 6 } & $0.5 \mathrm{~mL}$ & $\begin{array}{l}\text { Morphology } \\
\text { spherical } \\
\text { and irregular } \\
\text { shape }\end{array}$ & $\begin{array}{l}\text { Crystal } \\
\text { structure }\end{array}$ & particle size & $\begin{array}{l}\text { crystallite } \\
\text { size }\end{array}$ \\
\hline E & $1 \mathrm{~mL}$ & $\begin{array}{l}\text { roughly } \\
\text { spherical } \\
\text { shape }\end{array}$ & $\begin{array}{l}\text { cubic } \\
\text { structure }\end{array}$ & $17.9 \pm 3.4 \mathrm{~nm}$ & $9.4 \pm 1.0 \mathrm{~nm}$ \\
\hline B & $2 \mathrm{~mL}$ & $\begin{array}{l}\text { roughly } \\
\text { spherical } \\
\text { shape }\end{array}$ & $\begin{array}{l}\text { cubic } \\
\text { structure }\end{array}$ & $6.5 \pm 1.2 \mathrm{~nm}$ & $6.5 \pm 0.7 \mathrm{~nm}$ \\
\hline F & $4 \mathrm{~mL}$ & $\begin{array}{l}\text { roughly } \\
\text { spherical } \\
\text { shape }\end{array}$ & $\begin{array}{l}\text { cubic } \\
\text { structure }\end{array}$ & $12.5 \pm 2.0 \mathrm{~nm}$ & $12.3 \pm 1.0 \mathrm{~nm}$ \\
\hline G & $6 \mathrm{~mL}$ & $\begin{array}{l}\text { roughly } \\
\text { spherical } \\
\text { shape }\end{array}$ & $\begin{array}{l}\text { cubic } \\
\text { structure }\end{array}$ & $13.5 \pm 2.0 \mathrm{~nm}$ & $9.7 \pm 1.0 \mathrm{~nm}$ \\
\hline
\end{tabular}

The optical properties of SnTe nanoparticles with different size $(6.5 \pm 1.2,12.5$ $\pm 2.0 \mathrm{~nm}, 17.9 \pm 3.4 \mathrm{~nm}$ and $32.4 \pm 11.5 \mathrm{~nm}$ ) were investigated using a BOMEM DA8 spectrometer. The IR absorption measurements were made on drop-cast films of nanoparticles deposited on the $\mathrm{CaF}_{2}$ discs. Fig. 6.23 shows the IR absorption spectra for films of the four nanoparticle samples. The observed broad absorption peak around $3000 \mathrm{~nm}$ with a shoulder about $3400 \mathrm{~nm}$ on each spectrum, represent the vibrational modes of the TEA bound on the surface of SnTe nanoparticles. In addition, an absorption peak at $2270 \mathrm{~nm}(0.54 \mathrm{eV})$ was observed in the spectrum for the sample with the smallest particle size $(\sim 6.5$ $\mathrm{nm})$. This value is significantly higher than that the $0.18 \mathrm{eV}$ for the bulk band gap of $\mathrm{SnTe}^{30}$ and same to $0.54 \mathrm{eV}$ reported for $\sim 7.2 \mathrm{~nm}$ SnTe nanoparticles by Alivisatos's group. ${ }^{1}$ It was also reported that $14 \mathrm{~nm}$ SnTe nanoparticle has an absorption peak around $3150 \mathrm{~nm}(0.39 \mathrm{eV})$ which is in the same position 
of the O-H stretch of TEA. Therefore, the absorption peaks for the other three sample could not be identified. A blue shift in the optical band gap with reduced particle size was observed due to the quantum confinement effects. A photograph presented in Fig. 6.23 shows the colloidal SnTe nanoparticles dispersion in ethanol and forming a brown-black colour solution. In addition, no fluorescence was observed from the sample which may be due to the position of emission peak above the instrument detection limit $(2.5 \mu \mathrm{m})$.

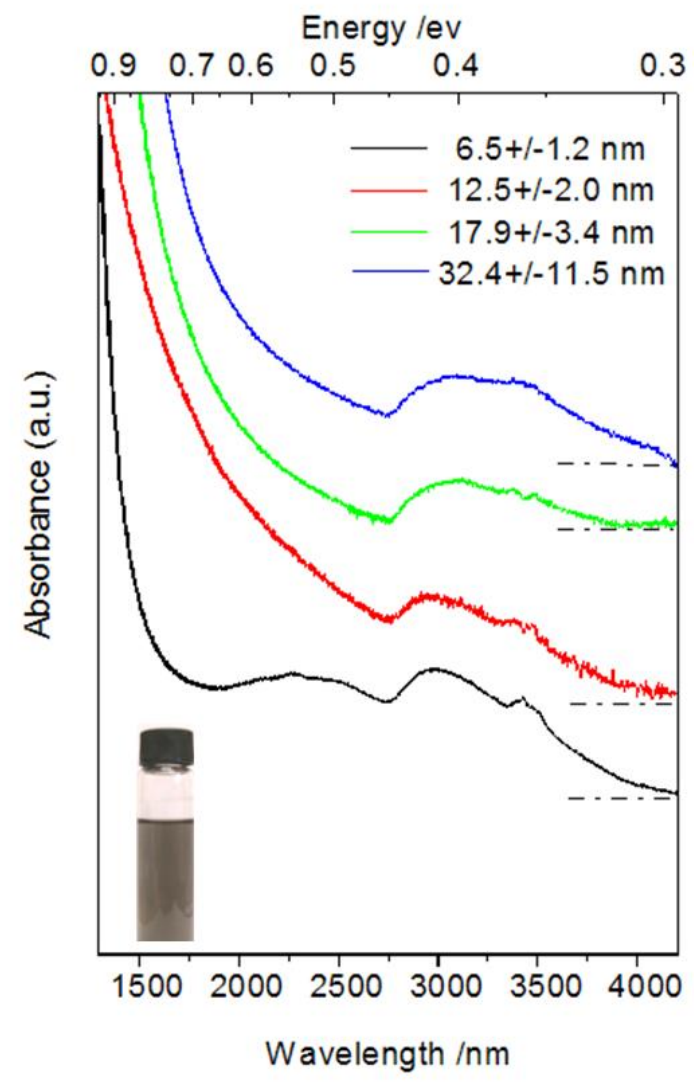

Fig. 6.23 IR absorption spectra of SnTe nanoparticle films. The dotted lines indicate zero-absorption. The inset: a photo of SnTe nanoparticles in ethanol.

\subsection{Synthesis of SnTe nanoparticles in $\mathrm{H}_{2} \mathrm{O}$}

In this series of experiments, SnTe nanoparticles were prepared by the reaction between $\mathrm{SnBr}_{2}$ and $\mathrm{NaHTe}$ in $\mathrm{H}_{2} \mathrm{O}$ in the presence of surfactants. As men- 
tioned in section 6.1, previous research has demonstrated that the stabilizing ligands and $\mathrm{pH}$ value of the solution play important roles in determining the particle size and size distribution. ${ }^{26,31-33}$

The molar ratio of $\mathrm{Sn} / \mathrm{Te} / \mathrm{RSH}$ was set as 1/0.5/2.4 following the literature. ${ }^{21,26}$ Two common thio-ligands, MPA and TGA, were used in the synthesis. For comparison, one experiment was performed in the presence of $2 \mathrm{~mL}$ TEA with the molar ratio of $\mathrm{Sn} / \mathrm{Te}$ precursors of $1 / 0.5$.

\subsubsection{Experiment $\mathrm{H}-\mathrm{J}$ (Effect of stabilizing ligands)}

\section{Experimental}

An initial mixture of $0.0279 \mathrm{~g}(0.1 \mathrm{mmol}) \mathrm{SnBr}_{2}$ (anhydrous, Sigma Aldrich) and the stabilizing ligand in $20 \mathrm{~mL}$ water produced a white precipitate. The white precipitate was slowly dissolved by dropwise addition of $1 \mathrm{M}$ solution of $\mathrm{NaOH}$. When the $\mathrm{pH}$ value reached 10 in the presence of TGA, the white precipitate was fully dissolved. When TGA was replaced by MPA or TEA, the precipitate did not dissolve until $\mathrm{pH}$ 12. In this set of experiments, the $\mathrm{pH}$ value was set as 12 .

In a separate round bottom flask, NaHTe aqueous solution was prepared by the reaction between Te powder (granules, 5+50 mesh, 99.99\%, Sigma Aldrich) and $\mathrm{NaBH}_{4}\left(\geq 98.5 \%\right.$,Sigma Aldrich) according to the literature. ${ }^{24}$ The freshly prepared NaHTe aqueous solution $(0.25 \mathrm{~mL}, 0.2 \mathrm{M})$ was swiftly added to an argon saturated $\mathrm{SnBr}_{2}$ solution with vigorous stirring at room temperature. The colour of the solution changed from colourless to black immediately. After 5-30 seconds, a black precipitate was formed. The reaction was allowed to continue at room temperature for 5 minutes. All the syntheses were carried 
out at room temperature under argon. The product was carefully separated by centrifugation and further washed with distilled water for three to four times to remove free ligands. The as-prepared samples were characterized using TEM, EDX, SAED, XRD and FTIR.

\subsubsection{Results}

Fig. 6.24a, c and d show typical LRTEM images of as-prepared products using TGA, MPA or TEA as a stabilizing agent, respectively. The as-prepared nanoparticles agglomerated, forming large clusters with ranges from $100 \mathrm{~nm}$ to $500 \mathrm{~nm}$. The HRTEM image clearly shows atomic lattice fringes of the asprepared SnTe nanoclusters (Fig. 6.24b), which demonstrates the highly crystalline nature of the products.

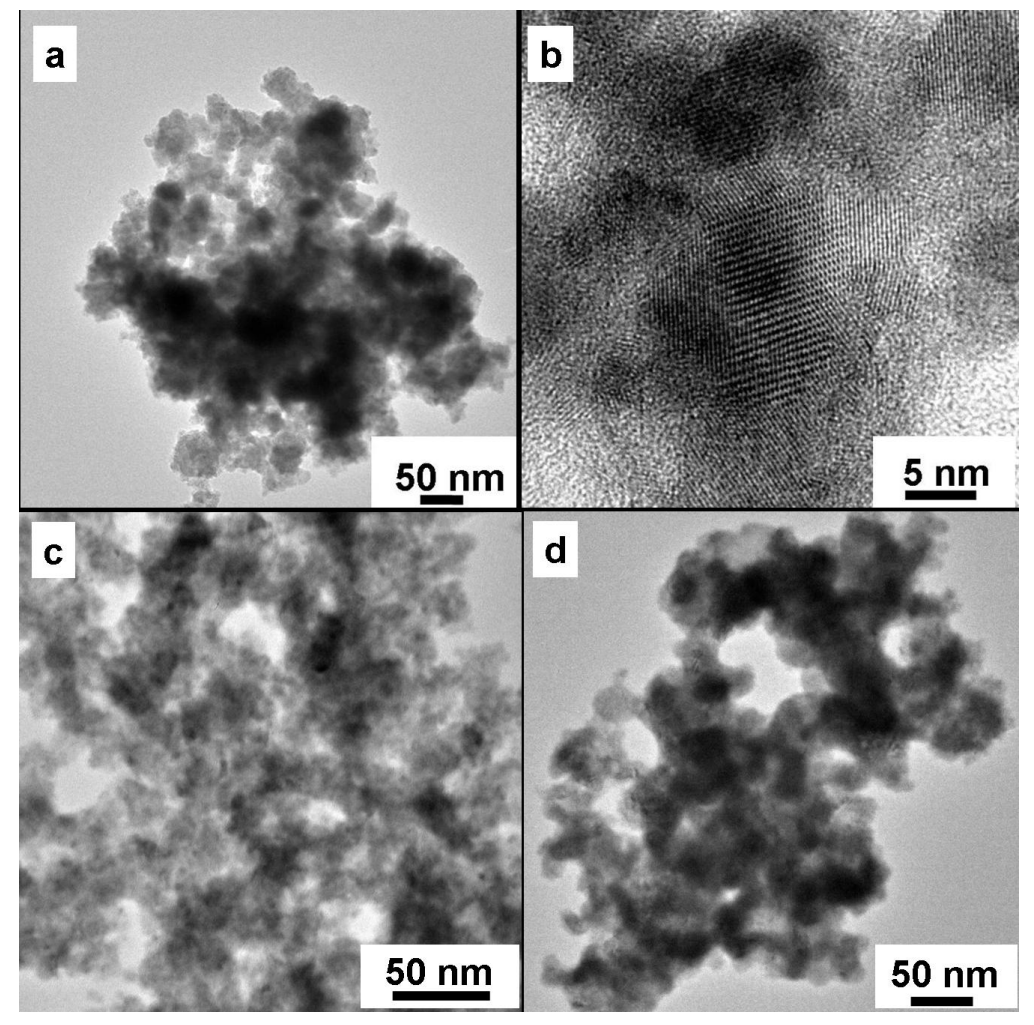

Fig. 6.24 (a),(b)Typical TEM images of SnTe nanoparticles synthesized in the presence of TGA. (c),(d) LRTEM images of SnTe nanoparticles synthesized in the presence of MPA and TEA, respectively. 
The crystal structure and chemical compositions for the as-prepared products were determined by XRD pattern and EDX spectrum. XRD analysis for the sample obtained from TGA , Fig. 6.25, showed no impurities, and all the peaks can be indexed to a cubic phase SnTe. EDX analysis, Fig. 6.26, revealed nearly stoichiometric composition of the SnTe nanoclusters. The same results were also obtained for the samples prepared from MPA and TEA.

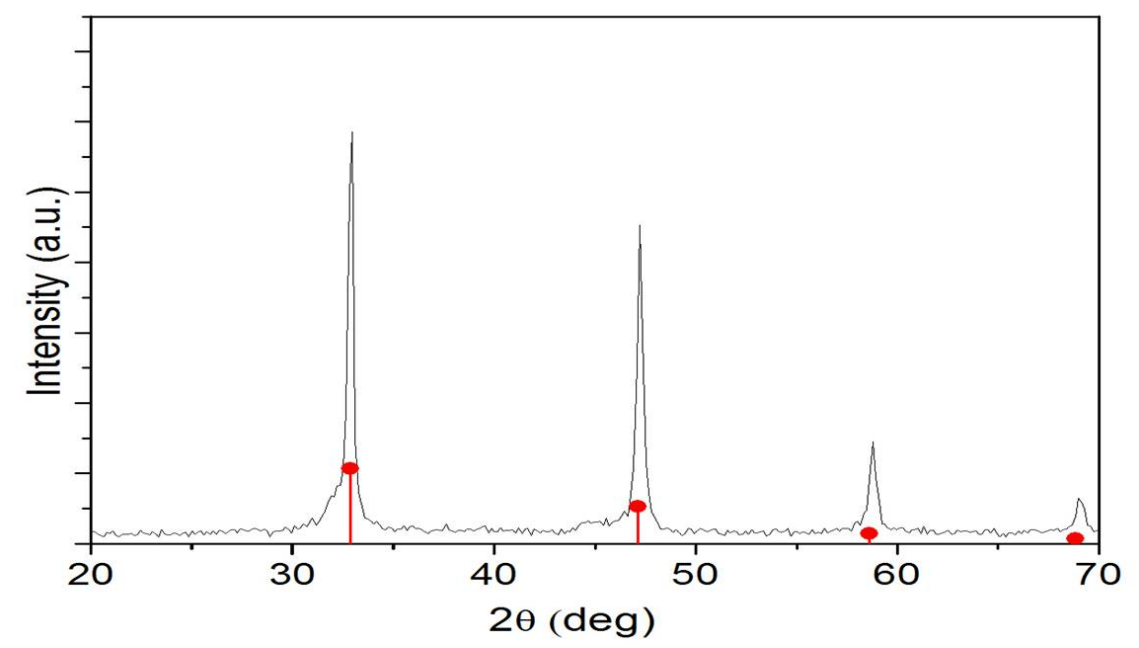

Fig. 6.25 XRD spectrum of product obtained from TGA (Co radiation). The expected positions and intensities for peaks corresponding to cubic SnTe (ICDD 00-046-1210) are marked by lines labeled with "•".

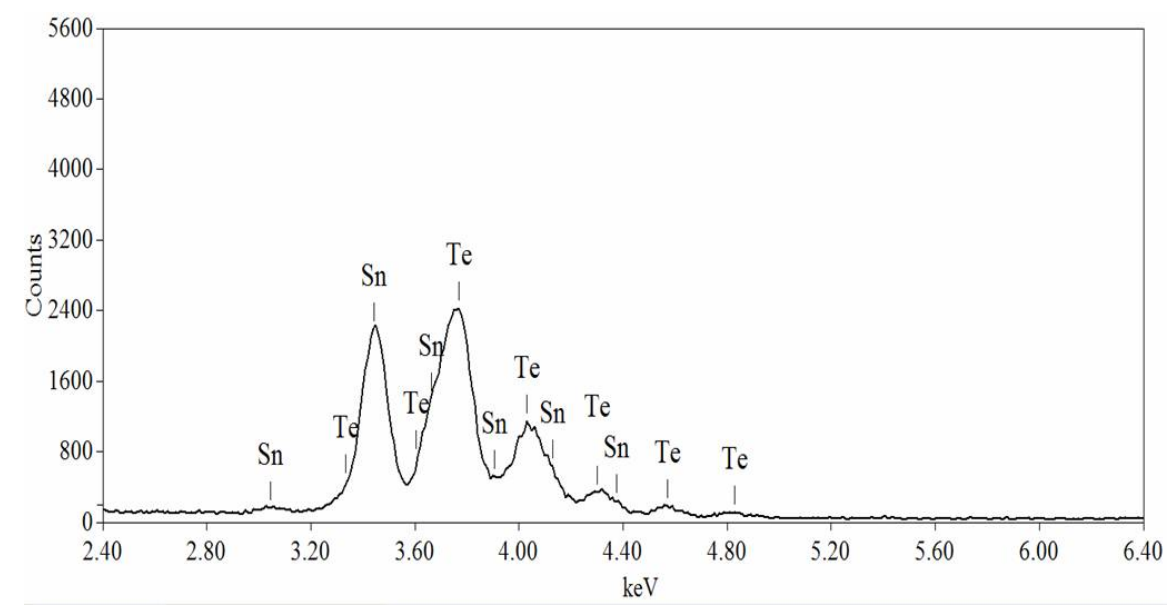

Fig. 6.26 EDX spectrum showing an atomic ratio of $S n$ to Te of 1:0.9.

The surface bonding of SnTe nanoparticles was characterized by FTIR spectroscopy and showed the presence of capping ligands (Fig. 6.27). The FTIR 
data obtained from different samples are summarized in Table 6.4.

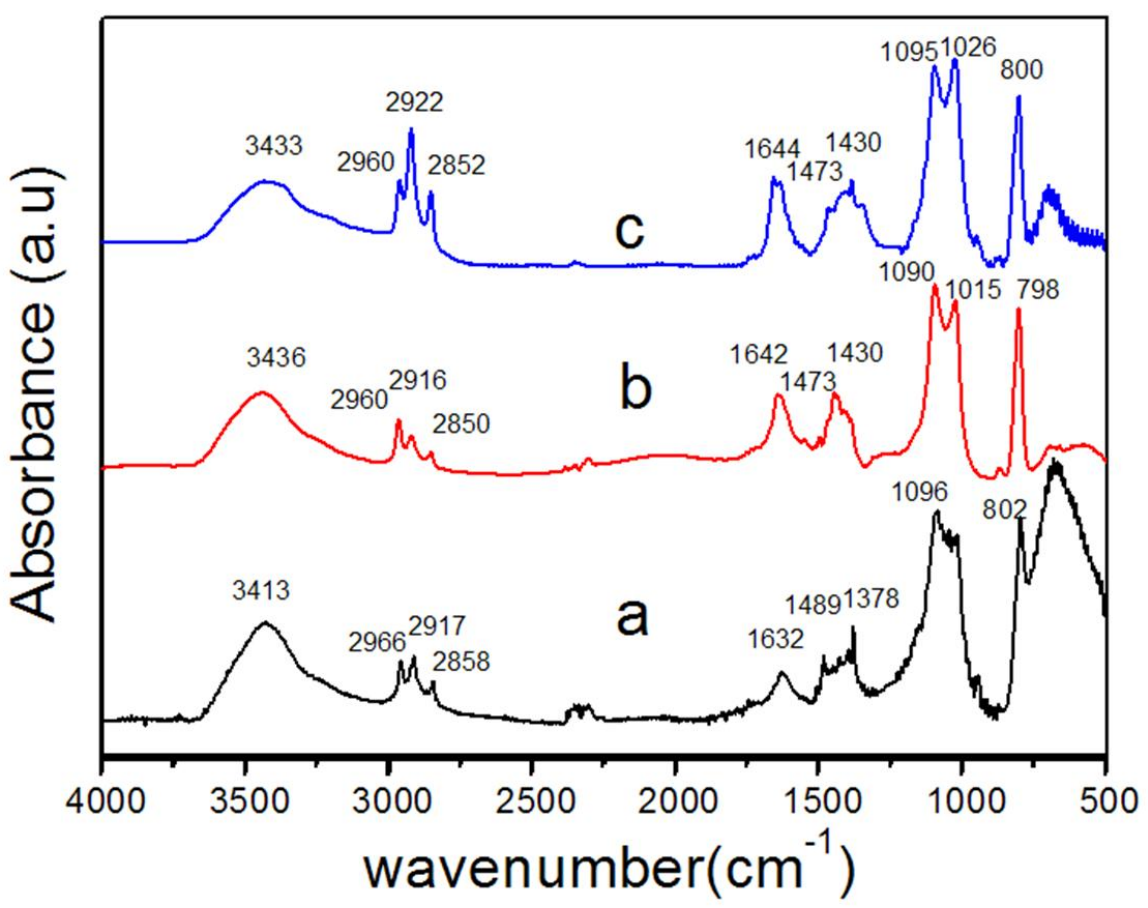

Fig. 6.27 FTIR spectra of (a) TEA-capped SnTe nanoparticles, (b) TGAcapped SnTe nanoparticles, and (c) MPA-capped SnTe nanoparticles.

Table 6.4 Assignment of the FTIR Bands (in $\mathrm{cm}^{-1}$ ) of Fig. 6.27.

\begin{tabular}{|l|l|l|l|}
\hline $\begin{array}{l}\text { TEA-capped } \\
\text { SnTe NPs }\end{array}$ & $\begin{array}{l}\text { TGA-capped } \\
\text { SnTe NPs }\end{array}$ & $\begin{array}{l}\text { MPA-capped } \\
\text { SnTe NPs }\end{array}$ & Assignment \\
\hline 3413 & 3436 & 3433 & $\nu(\mathrm{O}-\mathrm{H})+\mathrm{O}-\mathrm{H}\left(\mathrm{H}_{2} \mathrm{O}\right)$ \\
\hline $2966,2917,2858$ & $2960,2916,2850$ & $2960,2922,2852$ & $\nu(\mathrm{C}-\mathrm{H})$ \\
\hline- & 1642 & 1644 & $\nu(\mathrm{COO})$ \\
\hline 1632 & - & - & $\mathrm{H}-\mathrm{O}-\mathrm{H}$ \\
\hline 1489 & 1473 & 1473 & $\delta(\mathrm{C}-\mathrm{C})$ \\
\hline- & 1430 & 1430 & $\nu(\mathrm{COO})$ \\
\hline 1378 & - & - & $\delta(\mathrm{C}-\mathrm{H})$ \\
\hline 1096 & 1090 & 1095 & $\delta(\mathrm{C}-\mathrm{O})+\nu(\mathrm{C}-\mathrm{O})$ \\
\hline 800 & 798 & 800 & $\nu(\mathrm{C}-\mathrm{C})+\nu(\mathrm{C}-\mathrm{O})$ \\
\hline
\end{tabular}

Band assignments show a significant absorbance in the region between 3550 $\mathrm{cm}^{-1}$ and $3000 \mathrm{~cm}^{-1}$ which is due to the strong $\mathrm{O}-\mathrm{H}$ stretching vibrations of the ligand and physisorbed and chemisorbed $\mathrm{H}_{2} \mathrm{O}$. The bands in the region of 2830 $\mathrm{cm}^{-1}-2966 \mathrm{~cm}^{-1}$ correspond to the $\mathrm{C}-\mathrm{H}$ stretching vibrational mode. The band 
at $1642 \mathrm{~cm}^{-1}$ and $1644 \mathrm{~cm}^{-1}$ are due to asymmetric COO stretching vibrations typical of an ionic carboxylate group. ${ }^{34,35}$ The $1632 \mathrm{~cm}^{-1}$ band is due to $\mathrm{H}-\mathrm{O}-\mathrm{H}$ bending of $\mathrm{H}_{2} \mathrm{O}$ molecules. The band at $1430 \mathrm{~cm}^{-1}$ is due to the symmetric COO stretching. Other band assignments are shown in the Table 6.4.

\section{Discussion: Reaction in $\mathrm{H}_{2} \mathrm{O}$-Effect of stabilizing ligand}

The results summarized in Table 6.5 shows that there is no significant change for the samples obtained from the different surfactants. At $\mathrm{pH} 12$, only SnTe clusters were formed.

Table 6.5 Summary of results for SnTe NPs obtained using different stabilizing ligand.

\begin{tabular}{|c|c|c|c|}
\hline Exp & stabilizing ligand & $\mathrm{pH}$ & Products \\
\hline H & TGA & 12 & crystalline cluster $(100-300 \mathrm{~nm})$ \\
\hline I & MPA & 12 & crystalline cluster $(100-500 \mathrm{~nm})$ \\
\hline J & TEA & 12 & crystalline cluster $(100-500 \mathrm{~nm})$ \\
\hline
\end{tabular}

The white precipitate observed from the initial mixture of $\mathrm{SnBr}_{2}$ and MPA or TGA could be due to the formation of Sn-MPA or Sn-TGA complex similar to the Cd complex. ${ }^{36}$ These compounds are insoluble at low $\mathrm{pH}$ value depending on the ligands. Similar observation have been frequently reported in making CdTe nanoparticles from Cd-MPA. ${ }^{36}$ In addition, the white precipitate appeared in the mixture of $\mathrm{SnBr}_{2}$ and TEA in $\mathrm{H}_{2} \mathrm{O}$ may be due to the formation of $\mathrm{Sn}(\mathrm{OH})_{2}$ from hydrolysis under base condition as TEA dissolved in $\mathrm{H}_{2} \mathrm{O}$. By further increase in the $\mathrm{pH}, \mathrm{Sn}(\mathrm{OH})_{2}$ transform to $\mathrm{Sn}(\mathrm{OH})_{4}{ }^{2-}$, the white precipitate disappeared. The formation mechanisms for the synthesis of SnTe are still unknown, they will be explored in a future study.

\subsubsection{Experiment K-L (Effect of $\mathrm{pH}$ condition)}

In this set of experiments, the $\mathrm{pH}$ effect on the synthesis of SnTe nanoparticles was investigated. A pH range of 10-12 was examined. TGA was used as a 
stabilizing ligand. The results for $\mathrm{pH} 12$ using TGA have been reported in experiment $\mathrm{H}$.

\section{Results: Experiment K (pH 11)}

Fig. 6.28 shows a typical TEM image of SnTe nanoparticles prepared at $\mathrm{pH}$ 11. The obtained nanoparticles have a spherical shape. The inset, Fig. 6.28b, shows an HRTEM image of an individual nanocrystal with the atomic lattice fringes clearly visible, demonstrating the highly crystalline nature of the SnTe nanoparticles. A histogram, as shown in Fig. 6.28c, indicates an average nanoparticle size of $5.0 \pm 1.0 \mathrm{~nm}$. The SAED pattern,Fig. $6.28 \mathrm{~d}$, shows sharp diffraction rings corresponding to the cubic rock salt structure SnTe.

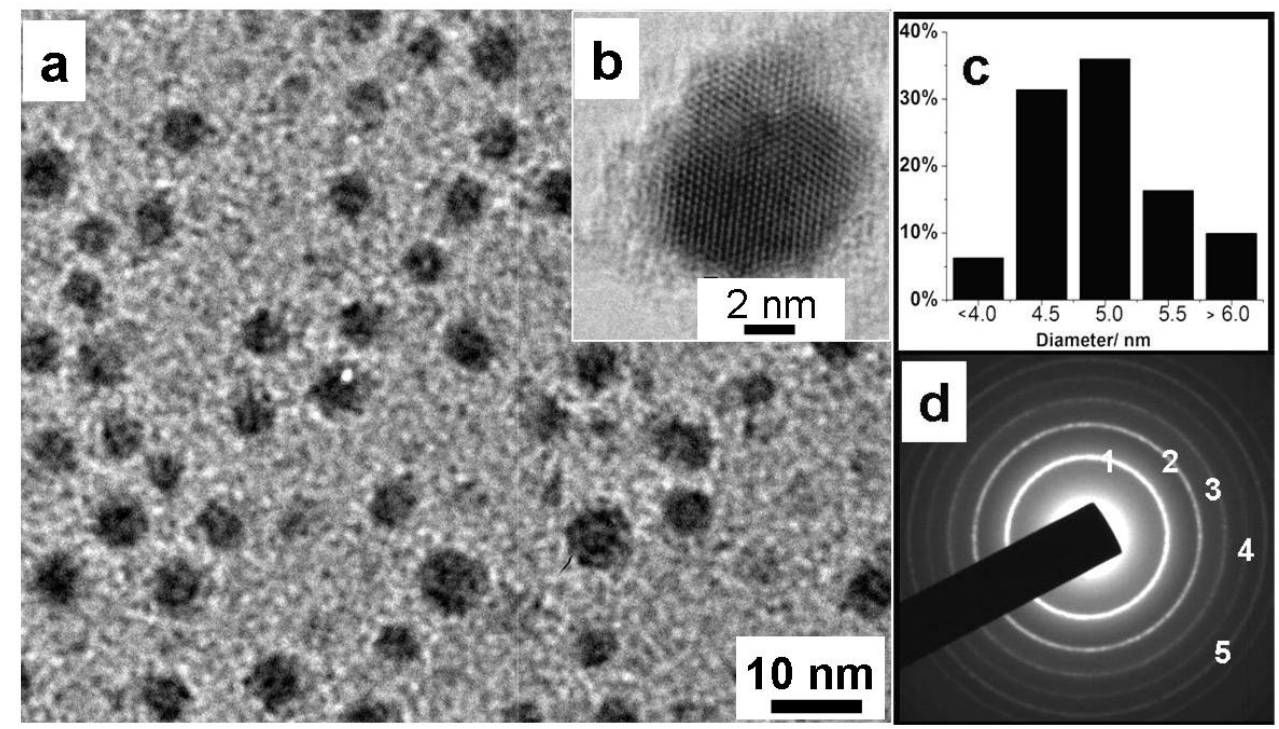

Fig. 6.28 Typical TEM images of nanoparticles obtained using TGA at $\mathrm{pH}$ 11 (a) and (b). (c) The corresponding histogram of particle size distribution, (d) SAED pattern of as-prepared SnTe nanoparticles.1-(200); 2-(220); 3-(222); 4-(400) and 5-(420).

\section{Results: Experiment L (pH 10)}

TEM image of the as-prepared SnTe nanoparticles is shown in Fig. 6.29a. The nanoparticles are $4.5 \pm 2.0 \mathrm{~nm}$ in size (Fig. 6.29b) and have rough spherical 
shape. SAED pattern indicates that the nanoparticles are cubic in structure (Fig. 6.29c).

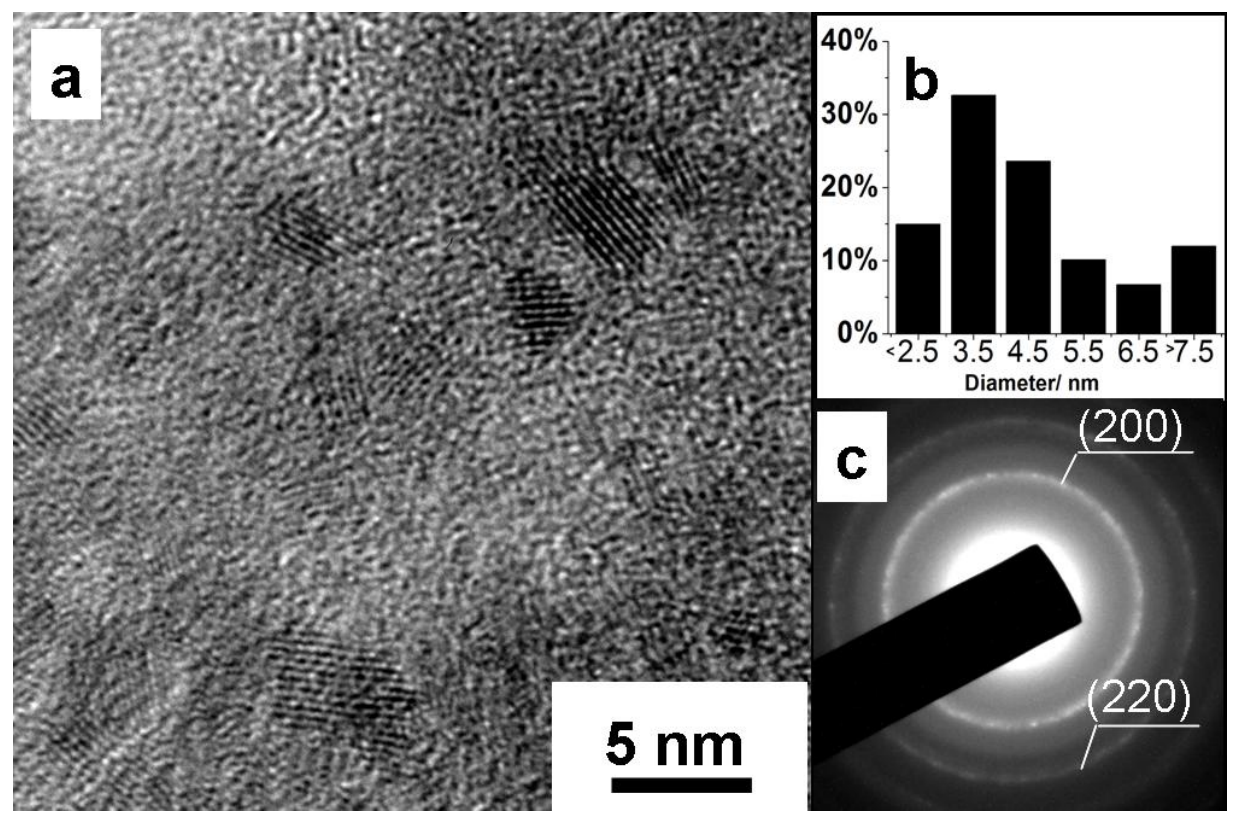

Fig. 6.29 (a)HRTEM image of nanocrystals obtained at $\mathrm{pH}$ 10,(b )histogram of nanoparticle size distribution, (c) SAED pattern of as-prepared SnTe nanoparticles

\section{Discussion of Reaction in $\mathrm{H}_{2} \mathrm{O}$ - Effect of $\mathrm{pH}$ condition}

The above experimental results (Table 6.6) shows that the optimum pH condition for the synthesis of SnTe nanoparticles was $\mathrm{pH}$ 11. $\mathrm{pH}$ plays a crucial role in the synthesis of size controlled SnTe nanoparticles. According to previous literature, the optimum $\mathrm{pH}$ value for the synthesis of CdTe depends strongly on the nature of the capping ligands and value of $\mathrm{pH} 11.2-11.8$ was recommend for TGA. For TGA, it was found that an increase of $\mathrm{pH}$ of the solution is followed by a considerable acceleration of the nanoparticles growth. ${ }^{31}$ 
Table 6.6 Summary of results for SnTe nanoparticles obtained at different $\mathrm{pH}$ conditions.

\begin{tabular}{|c|c|c|c|}
\hline Exp & stabilizing ligand & $\mathrm{pH}$ & Products \\
\hline H & TGA & 12 & crystalline cluster \\
\hline K & TGA & 11 & crystalline nanoparticles, average size $5.0 \pm 1.0 \mathrm{~nm}$ \\
\hline L & TGA & 10 & crystalline nanoparticles, average size $4.5 \pm 2.0 \mathrm{~nm}$ \\
\hline
\end{tabular}

\subsection{Summary}

In summary, water soluble SnTe nanoparticles of different sizes were synthesized from the reaction between $\mathrm{SnBr}_{2}$ and $\mathrm{NaHTe}$. The experiments were carried out in either DMF or $\mathrm{H}_{2} \mathrm{O}$.

In the first section, SnTe nanoparticles were produced in DMF, with TEA as the stabilizing ligand. The effect of reflux time on the crystallinity, size and size distribution of as-prepared nanoparticles has been investigated. The size of SnTe nanoparticles can be readily tuned from 2 to $30 \mathrm{~nm}$ by increasing the reflux time. Typically, to prepare crystalline SnTe nanoparticles, heat treatment (refluxing) is very important. In the present research, highly crystalline SnTe nanoparticles were obtained after a few minutes of reflux. In addition, the amount of TEA in the reaction system plays a crucial role in controlling the size of the SnTe nanoparticles. The experimental results revealed that the reaction at $50{ }^{\circ} \mathrm{C}$ with 2 minutes reflux in the presence of $2 \mathrm{~mL}$ TEA is the optimal reaction conditions for the synthesis of highly crystalline SnTe nanoparticles with a narrow size distribution. The energy of the absorption peak could be varied by turning the particles size. The optical band gap is $0.54 \mathrm{eV}$ for $\sim 6.5$ nm SnTe nanoparticle, making SnTe nanoparticles an promising material for mid-IR applications.

In the second section, SnTe nanoparticles were synthesized in $\mathrm{H}_{2} \mathrm{O}$. TEA, TGA 
and MPA were employed as surfactants. The surfactant and $\mathrm{pH}$ effects were studied. When the synthesis was carried out at $\mathrm{pH} 12$ using different surfactants, highly crystalline clusters with size ranges from 100 to $500 \mathrm{~nm}$ were obtained. When the $\mathrm{pH}$ decreased from 12 to 10 using TGA, roughly spherical shape SnTe nanoparticles with an average size around $5 \mathrm{~nm}$ were formed. The experimental results showed that nearly uniform SnTe nanoparticles with average size of $5.0 \pm 1.0 \mathrm{~nm}$ were formed using the water soluble TGA ligand at $\mathrm{pH} 11$. However, the synthesis mechanism is still not clear, the influence of the molar ratio of $\mathrm{Sn} / \mathrm{Te}$ sources, the influence of the molar ratio of $\mathrm{Sn} /$ ligand, the influence of reaction temperature will be investigated in the future work. Without completing the future study, it is hard to conclude the advantage or disadvantage of the aqueous technique. 


\section{REFERENCES}

[1] Kovalenko, M. V.; Heiss, W.; Shevchenko, E. V.; Lee, J.-S.; Schwinghammer, H.; Alivisatos, A. P.; Talapin, D. V. Journal of the American Chemical Society 2007, 129, 11354-11355.

[2] Patel, A. A.; Wu, F.; Zhang, J. Z.; Torres-Martinez, C. L.; Mehra, R. K.; Yang, Y.; Risbud, S. H. The Journal of Physical Chemistry B 2000, 104, 1159811605.

[3] Hickey, S. G.; Waurisch, C.; Rellinghaus, B.; Eychmuller, A. Journal of the American Chemical Society 2008, 130, 14978-14980.

[4] El-Sayed, M. A. Accounts of Chemical Research 2004, 37, 326-333.

[5] Klimov, V. I. The Journal of Physical Chemistry B 2006, 110, 16827-16845.

[6] Zhang, L.; Yin, L.; Wang, C.; lun, N.; Qi, Y.; Xiang, D. The Journal of Physical Chemistry C 2010, 114, 9651-9658.

[7] Son, S. J.; Bai, X.; Lee, S. B. Drug Discovery Today 2007, 12, 657-663.

[8] Yu, Z.; Lan, M.; Pei-Nan, W.; Si-Jia, L.; Ji-Yao, C.; Jia, G.; Wu-Li, Y.; ChangChun, W. Small 2008, 4, 777-780.

[9] Wing-Cheung, L.; Ken-Tye, Y.; Indrajit, R.; Hong, D.; Rui, H.; Weiwei, Z.; Paras, N. P. Small 2009, 5, 1302-1310.

[10] Delehanty, J. B.; Boeneman, K.; Bradburne, C. E.; Robertson, K.; Medintz, I. L. Expert Opinion on Drug Delivery 2009, 6, 1091-1112.

[11] Wang, W. Z.; Poudel, B.; Wang, D. Z.; Ren, Z. F. Advanced Materials 2005, $17,2110-2114$.

[12] Saini, R.; Pallavi,; Singh, M.; Kumar, R.; Jain, G. Chalcogenide letters 2010, 7, 197-202. 
[13] Mengui, U.; E.Abramof,; Rappl, P.; Ueta, A. Brazilian Journal of Physics 2006, 36, 324-327.

[14] Sabine, S.; Michael, B.; Lorenz, K. Inorganic Chemistry 2002, 41, 6001-6005.

[15] Mrinmoy, D.; Partha, S. G.; Vincent, M. R. Advanced Materials 2008, 20, $4225-4241$.

[16] Wang, Y.; Tang, Z.; Kotov, N. A. Materials Today 2005, 8, 20-31.

[17] Xu, Y.; Al-Salim, N.; Bumby, C. W.; Tilley, R. D. Journal of the American Chemical Society 2009, 131, 15990-15991.

[18] Wuister, S. F.; Meijerink, A. Journal of Luminescence 2003, 105, 35 - 43.

[19] Zhao, Y.; Li, Y.; Song, Y.; Jiang, W.; Wu, Z.; Wang, Y. A.; Sun, J.; Wang, J. Journal of Colloid and Interface Science 2009, 339, 336-343.

[20] Hua, H.; Huifeng, Q.; Chaoqing, D.; Kanglin, W.; Jicun, R. Angewandte Chemie 2006, 118, 7750-7753.

[21] Gao, M.; Kirstein, S.; Mohwald, H.; Rogach, A. L.; Kornowski, A.; Eychmuller, A.; Weller, H. The Journal of Physical Chemistry B 1998, 102, 83608363.

[22] Eng, J.; Song, X.; Li, L.; Qian, H.; Chen, K.; Xu, X.; Cao, C.; Ren, J. Talanta 2006, 70, 397-402.

[23] Rogach, A. L.; Franzl, T.; Klar, T. A.; Feldmann, J.; Gaponik, N.; Lesnyak, V.; Shavel, A.; Eychmller, A.; Rakovich, Y. P.; Donegan, J. F. The Journal of Physical Chemistry C 2007, 111, 14628-14637.

[24] Tellurium in organic synthesis, first edition ed.; Nicola Petragnani, H. A. S., Ed.; Elsevier Ltd., 2007; p 142. 
[25] Gaponik, N.; Talapin, D. V.; Rogach, A. L.; Hoppe, K.; Shevchenko, E. V.; Kornowski, A.; Eychmuller, A.; Weller, H. The Journal of Physical Chemistry B 2002, 106, 7177-7185.

[26] Zhang, H.; Wang, D.; Yang, B.; Mhwald, H. Journal of the American Chemical Society 2006, 128, 10171-10180.

[27] Lin, J.; Lin, Y.; Liu, P.; Meziani, M. J.; Allard, L. F.; Sun, Y.-P. Journal of the American Chemical Society 2002, 124, 11514-11518.

[28] Alden, H. R.; Oscar, H.; Jett, J., C. Arthur Journal of Polymer Science Part B: Polymer Letters 1971, 9, 503-507.

[29] Jun, Y.-w.; Lee, J.-H.; Choi, J.-s.; Cheon, J. The Journal of Physical Chemistry B 2005, 109, 14795-14806.

[30] Vassilev, V. S.; Vachkov, V. A.; Markova, I. N.; Petkov, P. Materials Letters 2001, 48, 104-108.

[31] Rogach, A. L.; Franzl, T.; Klar, T. A.; Feldmann, J.; Gaponik, N.; Lesnyak, V.; Shavel, A.; Eychmller, A.; Rakovich, Y. P.; Donegan, J. F. The Journal of Physical Chemistry C 2007, 111, 14628-14637.

[32] Aldana, J.; Wang, Y. A.; Peng, X. Journal of the American Chemical Society 2001, 123, 8844-8850.

[33] Shavel, A.; Gaponik, N.; Eychmller, A. The Journal of Physical Chemistry B 2004, 108, 5905-5908.

[34] Haris, P. I. Infrared Analysis of Peptides and Proteins; American Chemical Society, 1999; pp 54-95.

[35] Jun, M.-J.; Park, C. W.; Chol, S. R. Bull. Korean Chem. Soc. 1990, 11, 354-357. 
[36] Guo, J.; Yang, W.; Wang, C. The Journal of Physical Chemistry B 2005, 109, 17467-17473. 


\section{Chapter 7}

\section{Conclusions and Future Work}

\section{Contents}

7.1 Introduction . . . . . . . . . . . . . . 201

7.2 Tin Nanoparticles and Tin Germanium Nanowires . . . . . 202

7.3 Tin chalcogenide nanoparticles $\ldots \ldots \ldots \ldots$

\subsection{Introduction}

This Chapter presents the main conclusions of this thesis and suggests directions for future work. In this thesis, attention has been given to the development of new, low toxicity tin or germanium based semiconductor nanomaterials with a goal to replace cadmium based materials in infrared optical interference filter fabrication, solar cell industries and biomedical applications such as hyperthermal therapy. ${ }^{1,2}$ This research focuses on the novel synthesis and characterization of nanomaterials including tin germanium nanowires and tin chalcogenide nanoparticles. The developed novel synthetic approaches offer many advantages over the other methods developed in the past. This research shows, for the first time, the synthesis of one-dimensional tin germanium alloy nanowires. And, for the first time, it shows the synthesis of size controlled, 
water soluble tin monosulphide nanoparticles and tin telluride nanoparticles. The main contributions and summaries for each project are presented in the following sections.

For future work, some suggestions for the improvement of the synthesis proposed in this thesis and further investigation of optical and electrical properties of synthesized nanomaterials are discussed following the conclusion of each project. Also some ideas for future extension work is presented.

\subsection{Tin Nanoparticles and Tin Germanium Nanowires}

\subsubsection{Main contributions}

The most important contribution of this research is the development of a facile synthetic approach of tin nanoparticles (Chapters 3 ) and tin germanium nanowires (Chapter 4). The advantages of these methods have been summarized as follows:

\section{- Using high reduction temperature of tin halide}

Chemical reduction of inexpensive tin halide precursor to prepare tin nanoparticles was carried out at low temperature $\left(65^{\circ} \mathrm{C}\right.$, for 30 mins)in literature. ${ }^{3}$ The prepared tin nanoparticles exhibited a broad size distribution with average size around $40 \mathrm{~nm} .^{3}$

In contrast, high reduction temperature $\left(200{ }^{\circ} \mathrm{C}, 300{ }^{\circ} \mathrm{C}\right)$ and short reaction time (1 minute) used in this work produced smaller tin nanoparticles $(\sim 26$ $\mathrm{nm}$ ) with a narrow size distribution. This synthetic approach is simple and fast. The reaction conditions including reducing agent, reaction temperature 
and solvent and surfactant are same as that for the next step (tin germanium alloy nanowire synthesis). This offers an opportunity to make tin nanoparticles in situ and realize one-pot synthesis of tin germanium nanowires.

\section{- Using trioctylamine as a solvent}

Trioctylamine (TOA) was used as a solvent and surfactant. TOA has a high boiling point $\left(365^{\circ} \mathrm{C}\right)$ which enables tuning of the reaction temperature over a broad range.

\section{- Using solution phase synthesis of tin germanium alloys}

Previous research has demonstrated that the synthesis of tin germanium alloy is a significant challenge. ${ }^{4}$ There are few reports demonstrating the synthesis of bulk tin germanium alloy films and none of them produce the product in the nanoscale range size. ${ }^{4,5}$ Reported film synthesis techniques mainly used were molecular-beam epitaxy ${ }^{4}$ or chemical vapor deposition. ${ }^{5}$

In this work, tin germanium nanowires were synthesized through metal tin nanoparticles-assisted SLS growth. This work has explored a new method to prepare high yield and high quality tin germanium nanowires alloy based on solution phase synthesis.

- Using tin nanoparticles as tin source for the synthesis of tin germanium nanowires

Tin nanoparticles play a dual role in tin germanium alloy nanowire formation. It not only serves as a catalyst for nanowire growth but also can be utilized as the tin source for the formation of tin germanium alloy. 


\section{- Formation one dimensional tin germanium alloy nanowires}

one dimensional nanostructures offer the possibility of tuning the optical properties of tin germanium alloys by varying the diameter of the nanowires. This provides another research avenue to prepare tin germanium alloys with tunable band gaps.

\subsubsection{Tin nanoparticles}

To grow tin germanium nanowires via SLS mechanism, the first step was to synthesize metal tin nanoparticles. In Chapter 3, a simple, rapid and economical synthetic method was developed. Tin nanoparticles were prepared through a chemical reduction of low cost tin halides including $\mathrm{SnCl}_{2}$ or $\mathrm{SnI}_{4}$ with sodium borohydride at high temperature $\left(150^{\circ} \mathrm{C}-300{ }^{\circ} \mathrm{C}\right)$ for 1 minute. The general procedure employed was known as a "rapid hot injection" method. ${ }^{6}$

The size of tin nanoparticles can be tuned by varying reaction parameters including reaction temperature, precursor and surfactant. Reactions performed at different temperatures were found to produce similar average size particles but of different size distributions. At a lower temperature $\left(150{ }^{\circ} \mathrm{C}\right)$, the reaction rate is slow and consequently a low concentration of monomer is formed. This resulted in both slow nucleation and slow growth ${ }^{7}$ and the formation of a broader size distribution. While, at a higher reduction temperature $\left(300^{\circ} \mathrm{C}\right)$, both nucleation and the growth rate are too fast to be controlled which tends to form bigger particles with a broader size distribution. Reaction at $200{ }^{\circ} \mathrm{C}$ was shown to produce smaller particles with a narrow size distribution. In addition, the particle size was found to depend strongly on the tin precursor concentration as it increased along with increasing concentration of tin precursor. It was also found that changing the tin precursor significantly affected 
the particles size and size distribution. By replacing the $\mathrm{SnI}_{4}$ with $\mathrm{SnCl}_{2}$ in the reaction system, the particle size and size distribution increased dramatically. Adding an additional strong surfactant such as oleyamine (OLA) tends to produce smaller size tin nanoparticles with narrow size distribution.

The work presented in Chapter 3 provides a better understanding of the formation of tin nanoparticles and gives a solid fundation for the synthesis of tin germanium nanowires.

\subsubsection{Tin germanium nanowires}

Tin germanium nanowires were synthesized through a self-catalyzed process in which the nanowires were grown from tin nanoparticles via SLS growth. The tin nanoparticles were prepared either in situ or in advance following the method developed in Chapter 3. The synthetic approach used involves a reduction of tin halide with $\mathrm{NaBH}_{4}$ followed by a reduction of $\mathrm{Ge}(\mathrm{Ph})_{3} \mathrm{Cl}$ with $\mathrm{NaBH}_{4}$ in TOA.

The influence of reaction parameters on the formation of tin germanium nanowire was investigated, including injection rate, reaction temperature and molar ratio of tin/germanium precursors. It was found that the reducing agent injection process can affect the morphology of the nanowires. Straight nanowires were produced under a swift injection model while twisted nanowires were obtained by dropwise injection. Reaction temperature plays a crucial role in the generation of nanowires. A few short nanowires were formed at $200{ }^{\circ} \mathrm{C}$ while high yield, high quality, long nanowires were obtained at $300{ }^{\circ} \mathrm{C}$. High reaction temperature causes a higher surface diffusion rate leading to an enhanced growth rate of tin germanium nanowires. The molar ratio of tin/germanium precursors also affected the length of nanowires. The produced nanowires ex- 
hibit tapered ends. The composition of tin germanium nanowire obtained under optimal reaction condition using $\mathrm{SnI}_{4}$ and $\mathrm{Ge}(\mathrm{Ph})_{3} \mathrm{Cl}(1: 30)$ as precursors at $300{ }^{\circ} \mathrm{C}$ was examined by Powder X-ray Diffraction (XRD), Scanning Electron Microscopy(SEM) and Energy Dispersive X-ray Spectroscopy(EDX). The nanowires produced are homogeneous with a composition of $\mathrm{Sn}_{0.1} \mathrm{Ge}_{0.9}$.

The as-prepared tin germanium nanowires are highly crystalline with main growth direction along $<110>$. Some twinned tin germanium nanowires $(\sim$ $30 \%$ in total nanowires) of growth direction $<112>$ have been investigated by Transmission Electron Microscopy (TEM). The formation of twinned nanostructure has been discussed.

\subsubsection{Future work}

Future improvements should focus on the optimization of reaction conditions for growing uniform diameter tin germanium nanowires with diameter smaller than its Bohr radius with the goal of tuning the band gaps of tin germanium nanowires.

For future optoelectronics applications, the seed nanoparticles need to be removed from the nanowire core which may be achieved by careful sonication and separation. The electrical conductivity and optical properties of tin germanium nanowires (without nanoparticle seeds) is to be investigated in future studies.

This work has shown that it is possible to synthesize tin germanium nanowires via self-catalyzed SLS growth. The produced nanowires exhibit long length with high yield and good crystallinity. The synthetic approach described in this work provides many future possibilities for exploring germanium nanowire 
doping, diameter, length control and large-scale process.

\subsection{Tin chalcogenide nanoparticles}

\subsubsection{Main contributions}

The most important contribution of this research is the development of a novel, facile, one-pot synthetic route of water-soluble tin chalcogenide nanoparticles including SnS (Chapter 5) and SnTe (Chapter 6) nanoparticles. The advantages of this method have been summarized as follows:

\section{- Using tin halide compound as a tin precursor}

Previous research used the organometallic complex as $\mathrm{Sn}\left(\mathrm{Et}_{2} \mathrm{Dtc}\right)_{2}{ }^{8}$ and $\mathrm{Sn}\left[\mathrm{N}\left(\mathrm{SiMe}_{3}\right)_{2}\right]_{2}{ }^{9}$ as tin precursors for the synthesis of well size controlled tin chalcogenide nanoparticles. The latter precursor is air sensitive and expensive which may limit the development of later medium and large scale industrial process.

In contrast, the tin halide compound, $\mathrm{SnBr}_{2}$, used in this work is inexpensive, safe and easy to handle during the synthesis. The synthesis route offers great potential for a future scale-up process.

\section{- Using polar organic solvent}

To dissolve organometallic tin precursor, nonpolar organic solvents such as 1octadecene (ODE), oleylamine(OLA) and 1-hexadecene were frequently used in previous reaction systems. ${ }^{8}$ These reaction systems require that the reactants other than tin precursor involved in the synthesis should also be of good solubility in organic solvents. 
The use of polar organic solvents such as dimethylformamide (DMF), ethylene glycol (EG) and tetrahydrofuran (THF) allow the use of various ionic precursors that are readily available and easy to handle.

\section{- Using low reaction temperature}

Inorganic ionic precursors are more reactive than organometallic precursors. This allows carrying out the reactions at relatively low temperature which is advantageous in scaling-up these reactions.

\section{- Using triethanolamine as a surfactant}

Triethanolamine (TEA) is a multifunctional surfactant which is water soluble. It is the first time that this surfactant has been used for the synthesis of tin chalcogenide nanoparticles. This work demonstrates the ability of TEA to control the particle size. It can chelate $\mathrm{SnBr}_{2}$, forming an intermediate complex $\left[\mathrm{Sn}(\mathrm{TEA})_{n}\right]^{2+}$ and consequently control the nucleation and growth rate of the nanoparticles. ${ }^{10}$ Furthermore, it can bond strongly to the surface atoms of the nanoparticles which restricts particle growth and renders them water soluble.

The tin chalcogenide nanoparticles produced are readily dispersed in water for biomedical applications and other polar solvents for the incorporation of these IR-sensitive nanoparticles into biphase polymer blends for photovoltaic devices.

\subsubsection{Tin monosulphide}

Colloidal SnS nanoparticles (Chapter 5) of size in the $3-6 \mathrm{~nm}$ range have been synthesized through the ionic reaction between $\mathrm{SnBr}$ and $\mathrm{Na}_{2} \mathrm{~S}$ in the presence of ethanolamine in EG. 
The size of the SnS nanoparticles produced can be tuned from $\sim 3 \mathrm{~nm}$ to $\sim 6 \mathrm{~nm}$ by varying the reaction parameters such as different ethanolamine derivatives, the amount of TEA used and reflux time. Comparing the three ethanolamine ligands $\mathrm{N}, \mathrm{N}$-dimethylethanolamine (DMEA), triethanolamine (TEA)and N-methyldiethanolamine (MDEA), TEA was shown to provide the best size control, then MDEA followed by DMEA. This followed the number of $\mathrm{OH}$ groups in each surfactant. It was also found that the amount of TEA used in the synthesis affects the particle size and size distribution. The optimum amount of TEA needed was investigated. In addition, big size SnS nanoparticles can be produced through a refluxing process due to the Ostwald ripening.

Diffuse reflectance and absorbance measurements of the obtained SnS nanoparticles showed blue shift with decreasing of particle size due to the quantum confinement effects. SnS nanoparticles with size around $3 \mathrm{~nm}$ exhibit a reflectivity edge of $\sim 1.65 \mathrm{eV}$ which is significantly higher than the $1.1 \mathrm{eV}$ reported for the bulk band gap of SnS. ${ }^{11}$

\subsubsection{Tin telluride}

Following the successful synthetic approach developed for SnS nanoparticles, water soluble SnTe nanoparticles were, for the first time, obtained through the reaction between $\mathrm{SnBr}_{2}$ and NaHTe in the presence of TEA in DMF.

The size and size distributions of the SnTe nanoparticles produced were varied by changing the reaction parameters. It was found that the size of SnTe nanoparticles can be readily tuned from 2 to $30 \mathrm{~nm}$ by increasing the reflux time from $0 \mathrm{~min}$ to $5 \mathrm{~min}$. The amount of TEA used in the reaction system was found to play a crucial role in the SnTe nanoparticles growth process. The optimum amount of TEA required in the reaction system has been determined. 
A Bomem DA8 FTIR spectrometer was used in this work to record the absorption spectra in the near-mid IR region. The energy of the absorption peak was found to be varied with the particle size. The optical band gap is $0.54 \mathrm{eV}$ for $\sim 6.5 \mathrm{~nm}$ SnTe nanoparticles, which is significantly higher than the $0.18 \mathrm{eV}$ for bulk SnTe, ${ }^{12}$ making SnTe nanoparticles a promising material for mid-IR applications.

In addition, a number of exploratory experiments have been carried out in aqueous phase. TEA, thioglycolic acid (TGA) and 3-mercaptopropionic acid (MPA) were used as surfactants. It was found that the formation of SnTe clusters is ligand-independent at high $\mathrm{pH}(\mathrm{pH} 12)$ conditions. Decreasing the $\mathrm{pH}$ $(\mathrm{pH} \sim 10)$ and using thioglycolic acid (TGA) as a ligand was shown to give better size control. These results suggest potentially promising future research directions.

\subsubsection{Future work}

Future work for SnS projects should focus on the characterization of electrical properties of as-prepared $\mathrm{SnS}$ nanoparticles and using these nanoparticles in multi-junction solar cells.

Future work for SnTe projects should focus on the modification of the reaction conditions such as the molar ratio of tin and tellurium precursors, the molar ratio of tin precursor and ligands, reaction temperature and concentration of reactants. In particular, further work should also focus on the development of an aqueous synthesis of SnTe nanoparticles by using different thiol ligands, like thioglycerol, and different $\mathrm{pH}$.

The synthetic approach for the formation of water soluble tin monosulphide 
nanoparticles is facile, mild and easily controlled. The precursors, solvent and surfactant used are of low cost which has a potential for scale up. This synthetic approach has been used to produce water soluble SnTe nanoparticles. It could also be used for the future synthesis of SnSe nanoparticles. The use of multifunctional surfactant TEA could produce hydrophilic nanoparticles. It can be used for the future synthesis of water dispersible nanoparticles.

Furthermore, ternary nanoparticles may be prepared such as ZnSnTe taking the advantage of the similarity in the metal charge $(2+)$ and the cubic structure of $\mathrm{ZnTe}^{13}$ and SnTe. This could enable the tuning of the band gap closer to the visible region depending on the ratio of $\mathrm{Zn}: \mathrm{Sn}$. 


\section{REFERENCES}

[1] Wakeham, S.; Hawkins, G.; Henderson, G.; Carthey, N. Applied optics 2008, 47, C206-C213.

[2] Loo, C.; Lowery, A.; Halas, N.; West, J.; Drezek, R. Nano Letters 2005, 5, 709-711.

[3] Balan, L.; Schneider, R.; Billaud, D.; Ghanbaja, J. Materials Letters 2005, 59, $1080-1084$.

[4] He, G.; Atwater, H. A. Phys. Rev. Lett. 1997, 79, 1937-1940.

[5] Chizmeshya, A. V. G.; Bauer, M. R.; Kouvetakis, J. Chemistry of Materials 2003, 15, 2511-2519.

[6] Celso, d. M. D.; Peter, L.; Daniel, V. Small 2005, 1, 1152-1162.

[7] Yin, Y.; Alivisatos, A. P. Nature 2005, 437, 664-670.

[8] Koktysh, D. S.; McBride, J. R.; Rosenthal, S. J. Nanoscale Research Letter 2007, 2, 144-148.

[9] Hickey, S. G.; Waurisch, C.; Rellinghaus, B.; Eychmuller, A. Journal of the American Chemical Society 2008, 130, 14978-14980.

[10] Xu, Y.; Al-Salim, N.; Bumby, C. W.; Tilley, R. D. Journal of the American Chemical Society 2009, 131, 15990-15991.

[11] Albers, W.; Haas, C.; van der Maesen, F. Journal of Physics and Chemistry of Solids 1960, 15, 306-310.

[12] Kovalenko, M. V.; Heiss, W.; Shevchenko, E. V.; Lee, J.-S.; Schwinghammer, H.; Alivisatos, A. P.; Talapin, D. V. Journal of the American Chemical Society 2007, 129, 11354-11355. 
[13] Chemam, R.; Bouabellou, A.; Grob, J. J.; Muller, D.; Schmerber, G. Nuclear Instruments and Methods in Physics Research Section B: Beam Interactions with Materials and Atoms 2004, 216, 116-120. 